S1 Table. The information of EST-SSR primers of the study. (A) Dinucleotide repeat more than 9 times.

\begin{tabular}{l} 
Seq ID \\
\hline Locus 60 Transcript 55/59_Confidence 0.345 Length_3395
\end{tabular}

Locus_60_Transcript_55/59_Confidence_0.345_Length_3395 Locus 62 Transcript 8/14 Confidence 0.609 Length 2013

Locus_62_Transcript 8/14_Confidence 0.609 Length_2013

Locus_91_Transcript_7/37_Confidence_0.514_Length_1796

Locus_91_Transcript_7/37_Confidence_0.514_Length_1796

Locus_92_Transcript_52/102_Confidence_0.511_Length_3385

Locus 92_Transcript 52/102_Confidence_0.511_Length_3385

Locus_92_Transcript_52/102_Confidence_0.511_Length_3385

Locus_92_Transcript_52/102_Confidence_0.511_Length_3385

Locus_113_Transcript_53/68_Confidence_0.525_Length_2354

Locus_113_Transcript_53/68_Confidence_0.525_Length_2354

Locus_113_Transcript_53/68_Confidence_0.525_Length_2354

Locus_113_Transcript_53/68_Confidence_0.525_Length_2354

Locus_136_Transcript_15/18_Confidence_0.509_Length_1077

Locus_136_Transcript_15/18_Confidence_0.509_Length_1077

Locus_136_Transcript_15/18_Confidence_0.509_Length_1077 Locus_136_Transcript_15/18_Confidence_0.509_Length_1077

Locus_139_Transcript_63/70_Confidence_0.359_Length_1053

Locus 139 Transcript 63/70 Confidence 0.359 Length 1053 Locus_139_Transcript_63/70_Confidence_0.359_Length_1053 Locus_139_Transcript_63/70_Confidence_0.359_Length_1053 Locus_165_Transcript_47/93_Confidence_0.268_Length_2277

Locus 165 Transcript $47 / 93$ Confidence 0.268 Length 2277 Locus_190_Transcript_53/63_Confidence_0.522_Length_5794 Locus_190_Transcript_53/63_Confidence_0.522_Length_5794 Locus_190_Transcript_53/63_Confidence_0.522_Length_5794 Locus_190_Transcript 53/63_Confidence_0.522_Length_5794 Locus_190_Transcript_53/63_Confidence_0.522_Length_5794 Locus_190_Transcript_53/63_Confidence_0.522_Length_5794 Locus_190_Transcript_53/63_Confidence_0.522_Length_5794 Locus 190_Transcript 53/63 Confidence 0.522 Length 5794 Locus_194_Transcript_42/46_Confidence_0.556_Length_2713 Locus 194 Transcript 42/46 Confidence 0.556 Length 2713 Locus_220_Transcript_25/51_Confidence 0.552 Length 2237 Locus_220_Transcript_25/51_Confidence_0.552_Length_2237 Locus_288_Transcript_13/24_Confidence_0.527_Length_2899 Locus_288_Transcript_13/24_Confidence_0.527_Length_2899 Locus 314 Transcript $38 / 53$ Confidence 0.440 Length 3188 Locus_314_Transcript_38/53_Confidence_0.440_Length_3188 Locus_329_Transcript_38/56_Confidence_0.520_Length_2789 Locus_329_Transcript_38/56_Confidence_0.520_Length_2789

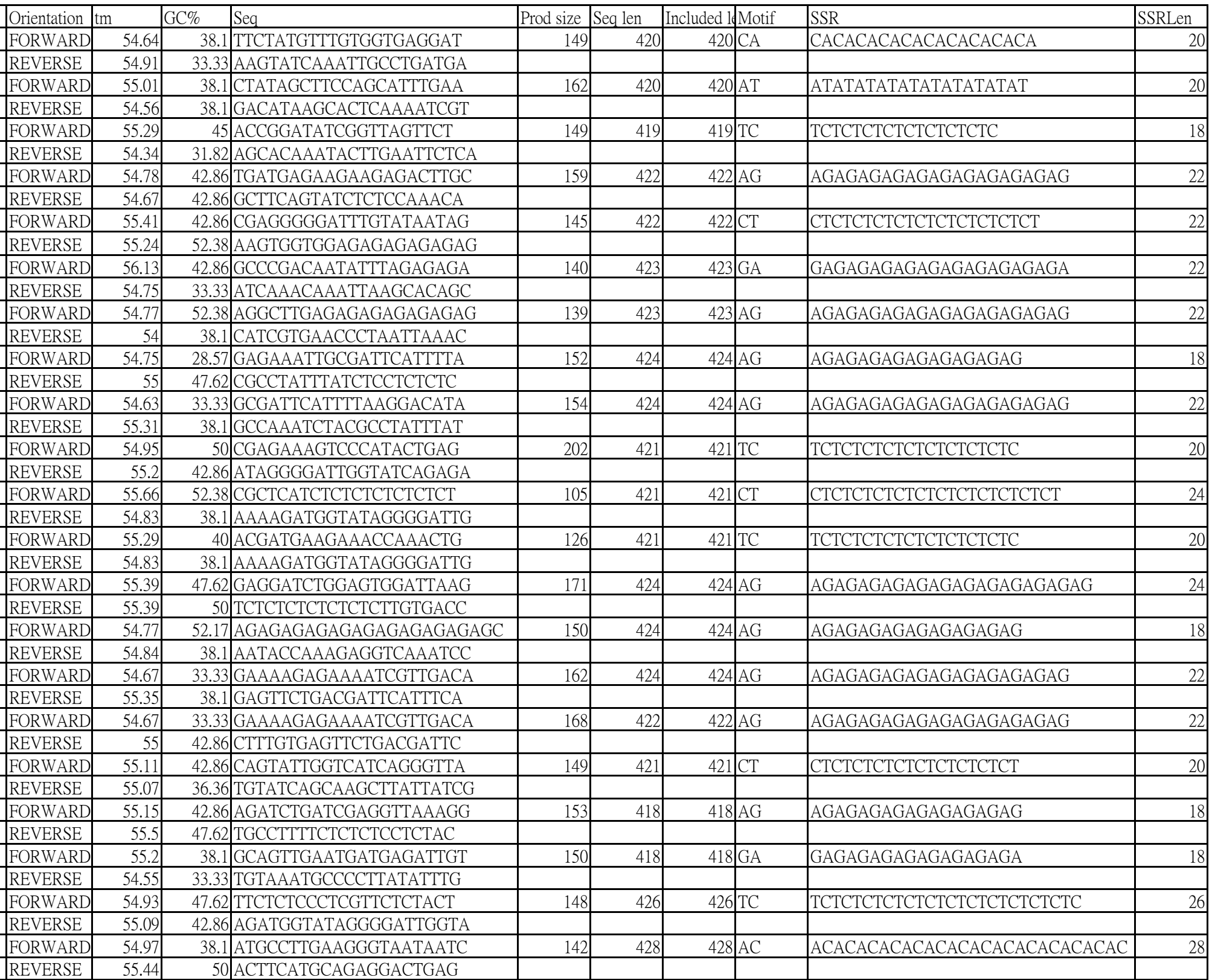


Locus_336_Transcript_10/11_Confidence_0.565_Length_774 Locus_336_Transcript_10/11_Confidence_0.565_Length_ 774 REVERSE FORWARD Locus_346_Transcript_190/203_Confidence_0.165_Length_3056 Locus_347_Transcript_16/29_Confidence_0.280_Length_4723 Locus_347 Transcript_16/29_Confidence 0.280_Length 4723 Locus_380_Transcript 80/90_Confidence 0.471_Length 3431 Locus_380_Transcript_80/90_Confidence_0.471_Length_3431 Locus_380_Transcript_80/90_Confidence_0.471_Length_3431 Locus 380 Transcript 80/90 Confidence 0.471 Length 3431 Locus_453_Transcript_95/260_Confidence_0.220_Length_2392 Locus_453_Transcript_95/260_Confidence_0.220_Length_2392 Locus_454_Transcript_27/50_Confidence_0.278_Length_2439 Locus_454_Transcript_27/50_Confidence_0.278_Length_2439 Locus_468_Transcript_159/168_Confidence_0.420_Length_3850 Locus_468_Transcript_159/168_Confidence_0.420_Length_3850 Locus_566_Transcript_10/17_Confidence_0.658_Length_6711 Locus_566_Transcript_10/17_Confidence_0.658_Length_6711

Locus_588_Transcript_139/151_Confidence_0.216_Length_2310 Locus_588_Transcript_139/151_Confidence_0.216_Length_2310 Locus_597_Transcript_640/643_Confidence_0.075_Length_3808 Locus_597_Transcript_640/643_Confidence_0.075_Length_3808 Locus_623_Transcript_90/105_Confidence_0.396_Length_2799 Locus_623_Transcript_90/105_Confidence_0.396_Length_2799 Locus_623_Transcript_90/105_Confidence_0.396_Length_2799 Locus_623_Transcript_90/105_Confidence_0.396_Length_2799 Locus 623 Transcript 90/105 Confidence 0.396 Length 2799 Locus_623_Transcript_90/105_Confidence_0.396_Length_2799 Locus_623_Transcript_90/105_Confidence_0.396_Length_2799 Locus_623_Transcript_90/105_Confidence_0.396_Length_2799 Locus 694 Transcript 18/125 Confidence 0.251 Length 1340 Locus_694_Transcript_18/125_Confidence_0.251_Length_1340 Locus_694_Transcript_18/125_Confidence_0.251_Length_1340 Locus_694_Transcript_18/125_Confidence_0.251_Length_1340 Locus_694_Transcript_18/125_Confidence_0.251_Length_1340 Locus_694_Transcript_18/125_Confidence_0.251_Length_1340 Locus_700_Transcript_59/63_Confidence_0.286_Length_2042 Locus_700_Transcript_59/63_Confidence 0.286 _Length_2042 Locus_721_Transcript_7/28_Confidence_0.618_Length_1364 Locus_721_Transcript_7/28_Confidence_0.618_Length_1364 Locus_725_Transcript_67/131_Confidence_0.450_Length_4072 Locus_725_Transcript_67/131_Confidence_0.450_Length_4072 Locus_725_Transcript_67/131_Confidence_0.450_Length_4072 Locus_725_Transcript_67/131_Confidence_0.450_Length_4072 Locus_743_Transcript 41/52_Confidence_0.489 Length 1468

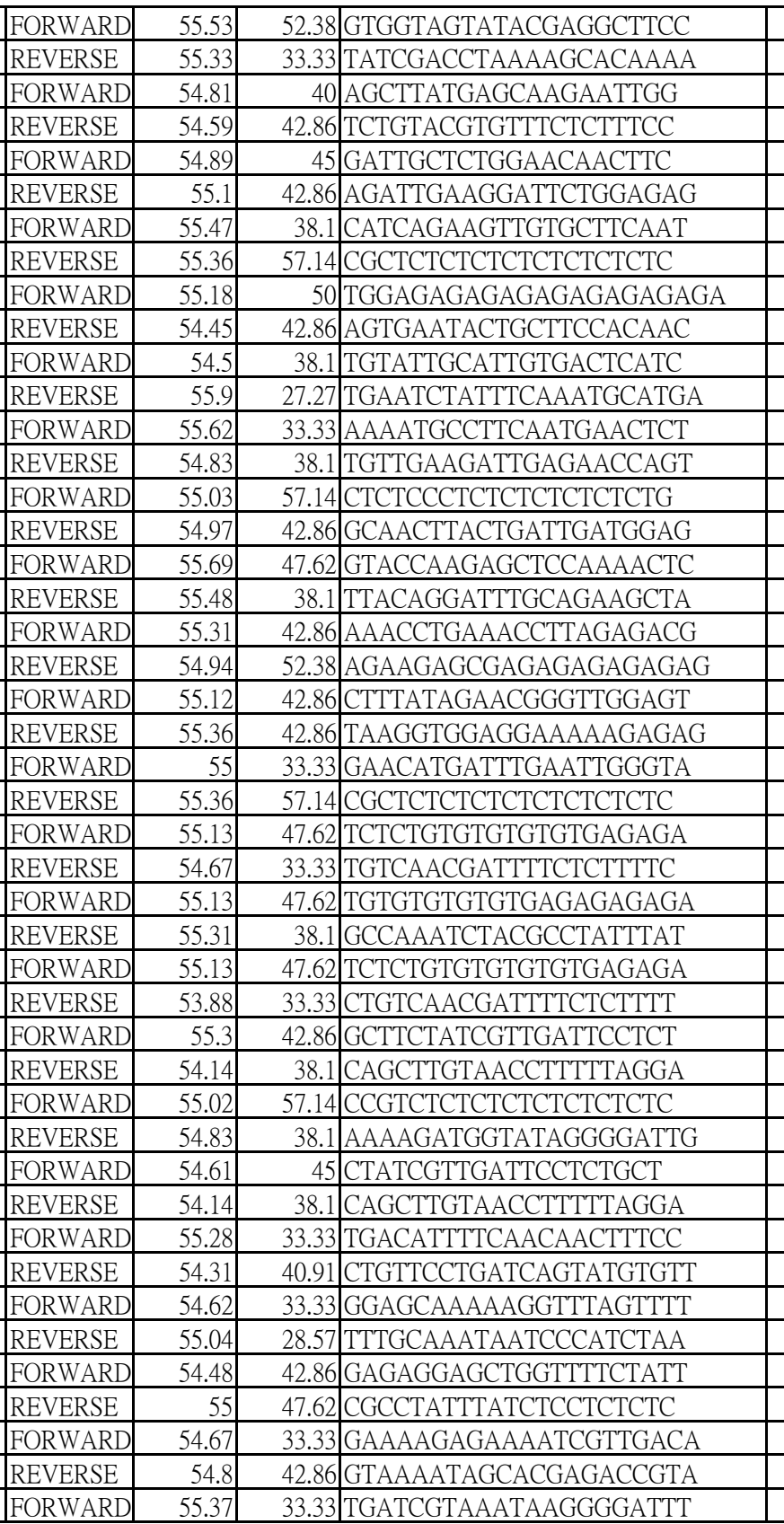

\begin{tabular}{l} 
I \\
I \\
\hline \\
\hline \\
\hline
\end{tabular}

\begin{tabular}{|c|c|c|c|c|c|}
\hline 152 & 421 & 421 & TC & TCTCTCTCTCTCTCTCTCTC & 20 \\
\hline 155 & 424 & 424 & $\mathrm{GA}$ & GAGAGAGAGAGAGAGAGAGAGAGA & 24 \\
\hline & & & & & \\
\hline 147 & 423 & 423 & TC & TСТСТСТСТСТСТСТСТСТСТС & 22 \\
\hline 146 & 424 & 424 & GA & GAGAGAGAGAGAGAGAGAGAGAGA & 24 \\
\hline & & & & & \\
\hline 150 & 424 & 424 & $\mathrm{AG}$ & AGAGAGAGAGAGAGAGAGAGAGAG & 24 \\
\hline & & & & & \\
\hline 134 & 418 & 418 & TG & TGTGTGTGTGTGTGTGTG & 18 \\
\hline 150 & 423 & 423 & TC & TCTCTCТСТСТСТСТСТСТСТС & 22 \\
\hline & & & & & \\
\hline 166 & 424 & 424 & CT & СТСТСТСТСТСТСТСТСТСТСТСТ & 24 \\
\hline 150 & 120 & 420 & $C T$ & СТСТСТСТСТСТСТСТСТСТ & 20 \\
\hline & & & & & \\
\hline 157 & 423 & 423 & $\mathrm{TC}$ & ТСТСТСТСТСТСТСТСТСТСТС & 22 \\
\hline 152 & 121 & 121 & $C \wedge$ & $G \triangle G \triangle G \triangle G \triangle G \triangle G \triangle G \triangle G \triangle G \triangle G A$ & 20 \\
\hline & & & & & \\
\hline 151 & 423 & 423 & $\mathrm{GA}$ & GAGAGAGAGAGAGAGAGAGAGA & 22 \\
\hline & & & & & 21 \\
\hline 151 & 423 & 423 & $A G$ & AGAGAGAGAGAGAGAGAGAGAGAG & 24 \\
\hline 200 & 423 & 423 & AG & AGAGAGAGAGAGAGAGAGAGAG & 22 \\
\hline & & & & & \\
\hline 152 & 423 & 423 & $\mathrm{AG}$ & AGAGAGAGAGAGAGAGAG & 18 \\
\hline 180 & 418 & 418 & $\mathrm{~T} C$ & ТСТСТСТСТСТСТСТСТС & 18 \\
\hline & & & & & \\
\hline 149 & 418 & 418 & CT & СТСТСТСТСТСТСТСТСТСТСТСТ & 24 \\
\hline & & & & & \\
\hline 176 & 424 & 424 & TC & TCTCTСТСТСТСТСТСТС & 18 \\
\hline & & & & & \\
\hline 150 & 419 & 419 & GA & GAGAGAGAGAGAGAGAGA & 18 \\
\hline & & & & & \\
\hline 134 & 419 & 419 & TA & TATATATATATATATATA & 18 \\
\hline & & & & & \\
\hline 142 & 424 & 424 & $\mathrm{AG}$ & AGAGAGAGAGAGAGAGAGAGAGAG & 24 \\
\hline & & & & & \\
\hline 143 & 424 & 424 & $\mathrm{AG}$ & AGAGAGAGAGAGAGAGAGAGAG & 22 \\
\hline & & & & & \\
\hline 164 & 419 & 419 & TC & TСТСТСТСТСТСТСТСТС & 18 \\
\hline
\end{tabular}


Locus_743_Transcript_41/52_Confidence_0.489_Length_1468 Locus_743_Transcript_41/52_Confidence_0.489_Length_1468 Locus_743_Transcript_41/52_Confidence_0.489_Length_1468 Locus_743_Transcript_41/52_Confidence_0.489_Length_1468 Locus_743_Transcript_41/52_Confidence_0.489_Length_1468 Locus 771_Transcript 43/152_Confidence 0.329_Length_1559 Locus_771_Transcript 43/152_Confidence_0.329_Length_1559 Locus_827_Transcript_9/19_Confidence_0.613_Length_1562 Locus_827_Transcript_9/19_Confidence_0.613_Length_1562 Locus_862_Transcript_66/186_Confidence_0.287_Length_3509 Locus 862 Transcript 66/186 Confidence 0.287 Length 3509 Locus_862_Transcript_66/186_Confidence_0.287_Length_3509 Locus_862_Transcript_66/186_Confidence_0.287_Length_3509 Locus_871_Transcript_10/52_Confidence_0.508_Length_3304 Locus_871_Transcript_10/52_Confidence_0.508_Length_3304 Locus_871_Transcript_10/52_Confidence_0.508_Length_3304 Locus_871_Transcript_10/52_Confidence_0.508_Length_3304 Locus_871_Transcript_10/52_Confidence_0.508_Length_3304 Locus_871_Transcript_10/52_Confidence_0.508_Length_3304 Locus_871_Transcript_10/52_Confidence_0.508_Length_3304 Locus_871_Transcript_10/52_Confidence_0.508_Length_3304 Locus_871_Transcript_10/52_Confidence_0.508_Length_3304 Locus_871_Transcript_10/52_Confidence_0.508_Length_3304 Locus_871_Transcript_10/52_Confidence_0.508_Length_3304 Locus_871_Transcript_10/52_Confidence_0.508_Length_3304 Locus_894_Transcript_36/57_Confidence_0.457_Length_2644 Locus 894_Transcript 36/57 Confidence 0.457 Length_2644 Locus_894_Transcript_36/57_Confidence_0.457_Length_2644 Locus_894_Transcript_36/57_Confidence_0.457_Length_2644 Locus_894_Transcript_36/57_Confidence_0.457_Length_2644 Locus 894 Transcript $36 / 57$ Confidence 0.457 Length 2644 Locus_894_Transcript_36/57_Confidence_0.457_Length_2644 Locus_894_Transcript_36/57_Confidence_0.457_Length_2644 Locus_894_Transcript_36/57_Confidence_0.457_Length_2644 Locus_894_Transcript_36/57_Confidence_0.457_Length_2644 Locus_894_Transcript_36/57_Confidence_0.457_Length_2644 Locus_894_Transcript_36/57_Confidence_0.457_Length_2644 Locus_977_Transcript_43/65_Confidence_0.481_Length_2237 Locus_977_Transcript_43/65_Confidence_0.481_Length_2237 Locus_977_Transcript_43/65_Confidence_0.481_Length_2237 Locus_977_Transcript_43/65_Confidence_0.481_Length_2237 Locus_999_Transcript 23/24_Confidence_0.606 Length_905 Locus_999_Transcript_23/24_Confidence_0.606_Length_905 Locus_999_Transcript_23/24_Confidence_0.606_Length_905 Locus 999_Transcript 23/24 Confidence 0.606 Length 905

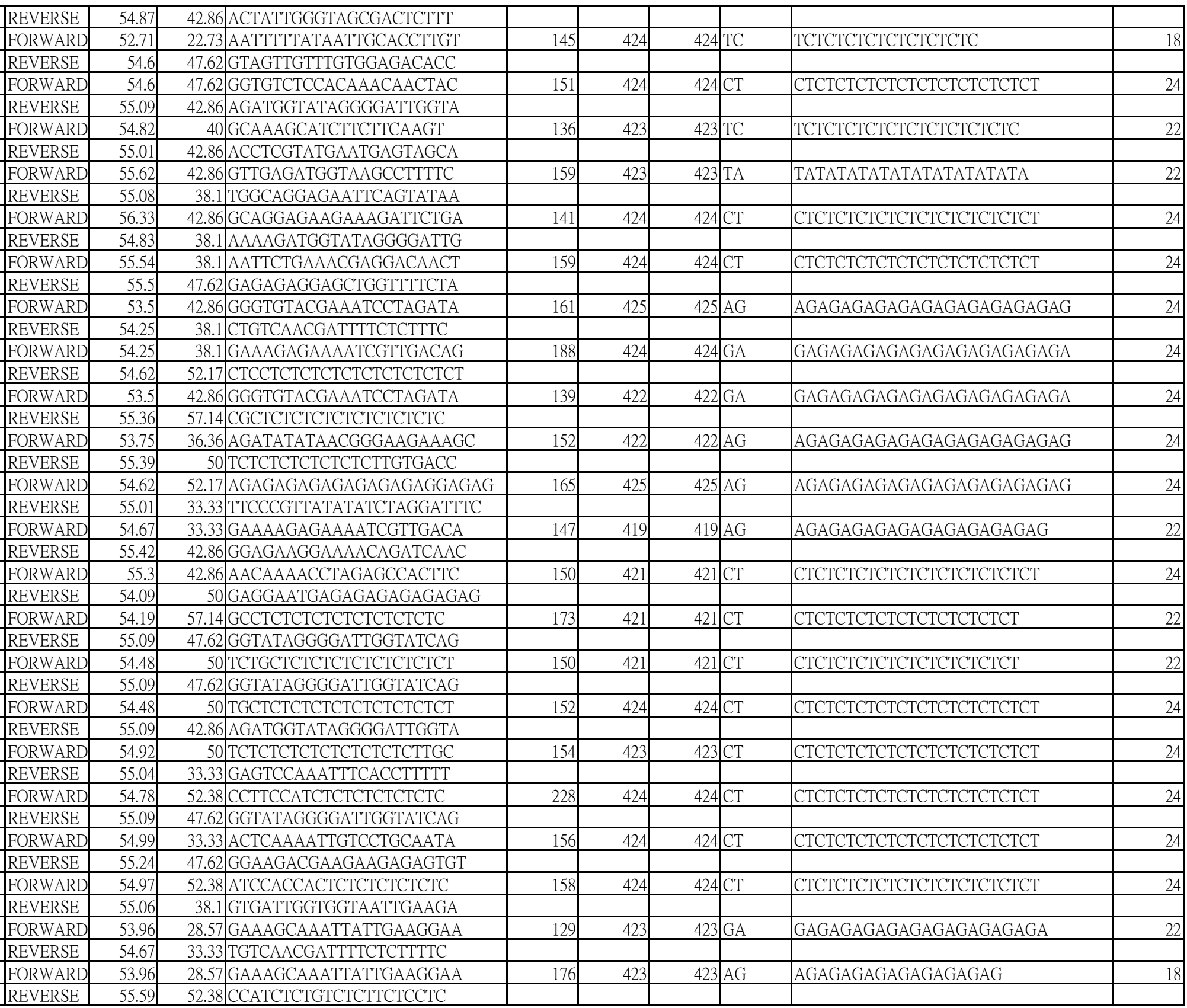


Locus_999_Transcript_23/24_Confidence_0.606_Length_905 Locus 999_Transcript 23/24_Confidence_0.606_Length 905 Locus_1000_Transcript_8/39_Confidence_0.605_Length_2175 Locus_1000_Transcript_8/39_Confidence_0.605_Length_2175 Locus_1000_Transcript_8/39_Confidence_0.605_Length_2175 Locus_1000_Transcript_8/39_Confidence_0.605_Length_2175 Locus_1003_Transcript_18/20_Confidence_0.642_Length_2519 Locus_1003_Transcript_18/20_Confidence_0.642_Length_2519 Locus_1025_Transcript_34/46_Confidence_0.464_Length_1343 Locus_1025_Transcript_34/46_Confidence_0.464_Length_1343 Locus 1030_Transcript 29/64_Confidence_0.335_Length_2552 Locus_1030_Transcript_29/64_Confidence_0.335_Length_2552 Locus_1069_Transcript_41/64_Confidence_0.406_Length_2993 Locus_1069_Transcript_41/64_Confidence_0.406_Length_2993 Locus_1100_Transcript_24/51_Confidence_0.515_Length_1753 Locus_1100_Transcript_24/51_Confidence_0.515_Length_1753 Locus_1139_Transcript_15/20_Confidence_0.702_Length_3598 Locus_1139_Transcript_15/20_Confidence_0.702_Length_3598 Locus_1156_Transcript_76/96_Confidence_0.242_Length_1523 Locus_1156_Transcript_76/96_Confidence_0.242_Length_1523 Locus_1156_Transcript_76/96_Confidence_0.242_Length_1523 Locus_1156_Transcript_76/96_Confidence_0.242_Length_1523 Locus_1184_Transcript_24/68_Confidence_0.468_Length_2356 Locus_1184_Transcript_24/68_Confidence_0.468_Length_2356 Locus_1184_Transcript_24/68_Confidence_0.468_Length_2356 Locus_1184_Transcript_24/68_Confidence_0.468_Length_2356 Locus 1210 Transcript $12 / 25$ Confidence 0.653 Length 1225 Locus_1210_Transcript_12/25_Confidence_0.653_Length_1225 Locus_1239_Transcript_30/64_Confidence_0.378_Length_2745 Locus_1239_Transcript_30/64_Confidence_0.378_Length_2745 Locus 1260 Transcript 25/44 Confidence 0.630 Length 3073 Locus_1260_Transcript_25/44_Confidence_0.630_Length_3073 Locus_1260_Transcript_25/44_Confidence_0.630_Length_3073 Locus_1260_Transcript_25/44_Confidence_0.630_Length_3073 Locus_1307_Transcript_93/117_Confidence_0.311_Length_2543 Locus_1307_Transcript_93/117_Confidence_0.311_Length_2543 Locus_1359_Transcript_6/47_Confidence_0.486_Length_1386 Locus_1359_Transcript_6/47_Confidence_0.486_Length_1386 Locus_1359_Transcript_6/47_Confidence_0.486_Length_1386 Locus_1359_Transcript_6/47_Confidence_0.486_Length_1386 Locus_1516_Transcript_52/110_Confidence_0.531_Length_2879 Locus_1516_Transcript_52/110_Confidence_0.531_Length_2879 Locus 1598 Transcript 49/127 Confidence 0.358 Length 19

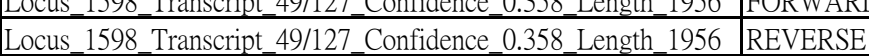
Locus_1609_Transcript_17/42_Confidence_0.582 Length 820

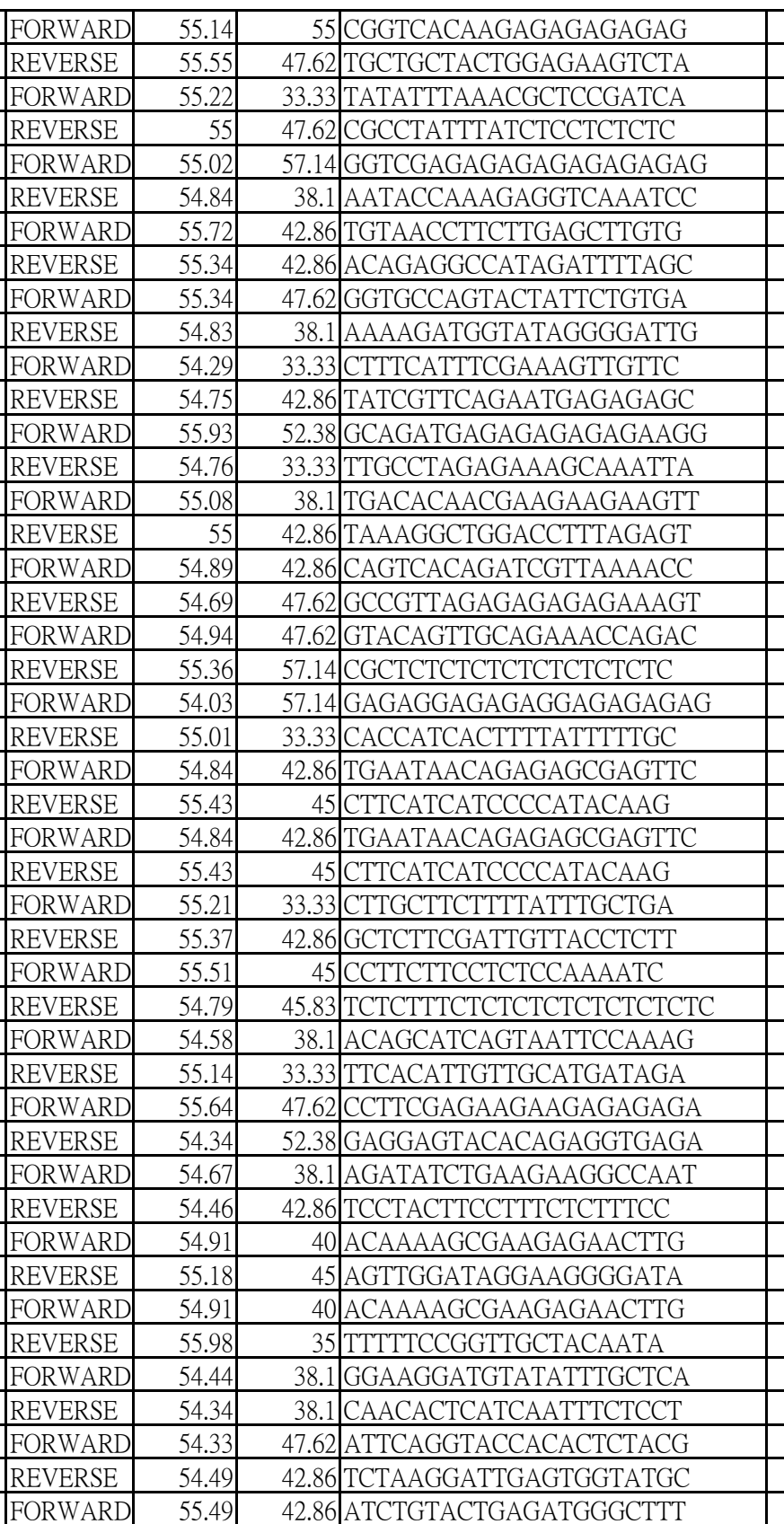

\begin{tabular}{|c|c|c|c|c|c|}
\hline 141 & 423 & 423 & $\mathrm{AG}$ & AGAGAGAGAGAGAGAGAGAGAG & 22 \\
\hline 145 & 425 & 425 & GA & GAGAGAGAGAGAGAGAGAGAGAGA & 24 \\
\hline & & & & & \\
\hline 150 & 425 & 425 & $\mathrm{AG}$ & AGAGAGAGAGAGAGAGAG & 18 \\
\hline & & & & & \\
\hline 152 & 418 & 418 & TC & TCTCTCTCTCTCTCTCTC & 18 \\
\hline & & & & & \\
\hline 140 & 424 & 424 & CT & СТСТСТСТСТСТСТСТСТСТСТСТ & 24 \\
\hline & & & & & \\
\hline 144 & 422 & 422 & CT & СТСТСТСТСТСТСТСТСТСТСТ & 22 \\
\hline 150 & 423 & 423 & $\mathrm{TC}$ & TСТСТСТСТСТСТСТСТСТСТС & 22 \\
\hline & & & & & \\
\hline 142 & 420 & 420 & $\mathrm{AG}$ & AGAGAGAGAGAGAGAGAGAG & 20 \\
\hline & & & & & \\
\hline 183 & 419 & 419 & CT & СТСТСТСТСТСТСТСТСТ & 18 \\
\hline 152 & & & & & \\
\hline 153 & 424 & 424 & $A G$ & AGAGAGAGAGAGAGAGAGAGAGAG & 24 \\
\hline 150 & 424 & 424 & $\mathrm{AG}$ & \begin{tabular}{|l} 
AGAGAGAGAGAGAGAGAGAGAGAG \\
\end{tabular} & 24 \\
\hline & & & & & \\
\hline 153 & 425 & 425 & GA & GAGAGAGAGAGAGAGAGAGAGAGA & 24 \\
\hline & & & & & \\
\hline 153 & 421 & 421 & GA & GAGAGAGAGAGAGAGAGAGAGAGA & 24 \\
\hline & & & & & \\
\hline 150 & 418 & 418 & $\mathrm{AG}$ & AGAGAGAGAGAGAGAGAG & 18 \\
\hline & & & & & \\
\hline 164 & 419 & 419 & AG & AGAGAGAGAGAGAGAGAG & 18 \\
\hline & & & & & \\
\hline 145 & 422 & 422 & TA & TATATATATATATATATATATA & 22 \\
\hline & & & & & \\
\hline 151 & 422 & 422 & CT & СТСТСТСТСТСТСТСТСТСТСТ & 22 \\
\hline & & & & & \\
\hline 165 & 421 & 421 & TC & TСТСТСТСТСТСТСТСТСТС & 20 \\
\hline & & & & & \\
\hline 168 & 420 & 420 & TA & TATATATATATATATATATA & 20 \\
\hline & & & & & \\
\hline 194 & 420 & 420 & TA & TATATATATATATATATATATATA & 24 \\
\hline & & & & & \\
\hline 154 & 424 & 424 & CT & СТСТСТСТСТСТСТСТСТСТСТСТ & 24 \\
\hline & & & & & \\
\hline 161 & 419 & 419 & $\mathrm{AC}$ & ACACACACACACACACAC & 18 \\
\hline & & & & & \\
\hline 148 & 423 & 423 & GA & GAGAGAGAGAGAGAGAGAGAGA & 22 \\
\hline
\end{tabular}


Locus_1609_Transcript_17/42_Confidence_0.582_Length_820 Locus_1609_Transcript_17/42_Confidence 0.582 Length 820 Locus_1609_Transcript_17/42_Confidence_0.582_Length_820 Locus_1633 Transcript 29/35_Confidence 0.365_Length_2130 Locus_1633_Transcript_29/35_Confidence_0.365_Length_2130 Locus_1633 Transcript 29/35 Confidence 0.365 Length 2130 Locus_1633_Transcript 29/35_Confidence_0.365_Length_2130 Locus_1633_Transcript_29/35_Confidence_0.365_Length_2130 Locus_1633_Transcript_29/35_Confidence_0.365_Length_2130 Locus_1633_Transcript_29/35_Confidence_0.365_Length_2130 Locus_1633_Transcript 29/35_Confidence 0.365_Length_2130 Locus_1644_Transcript_32/35_Confidence_0.608_Length_3704 Locus_1644_Transcript_32/35_Confidence_0.608_Length_3704 Locus_1644_Transcript_32/35_Confidence_0.608_Length_3704 Locus_1644_Transcript 32/35_Confidence_0.608_Length_3704 Locus_1644_Transcript_32/35_Confidence_0.608_Length_3704 Locus_1644_Transcript_32/35_Confidence_0.608_Length_3704 Locus_1656_Transcript_56/66_Confidence_0.457_Length_1455 Locus 1656 Transcript 56/66 Confidence 0.457 Length 1455 Locus_1656_Transcript_56/66_Confidence_0.457_Length_1455 Locus_1656_Transcript_56/66_Confidence_0.457_Length_1455 Locus_1675_Transcript_29/41_Confidence_0.505_Length_2360 Locus 1675 Transcript 29/41 Confidence 0.505 Length 2360 Locus_1681_Transcript_75/91_Confidence_0.392_Length_2256 Locus_1681_Transcript_75/91_Confidence_0.392_Length_2256 Locus 1783 Transcipt 105/138 Confidence 0.339 Length 5 Locus_1806_Transcript_28/33_Confidence_0.521_Length_1557 Locus_1806_Transcript_28/33_Confidence_0.521_Length_1557 Locus_1861_Transcript_32/77_Confidence_0.481_Length_2754 Locus 1861 Transcript 32/77 Confidence 0.481 Length 2754 Locus_1912_Transcript_21/23_Confidence_0.333_Length_536 Locus_1912_Transcript_21/23_Confidence_0.333_Length_536

Locus_1930_Transcript_47/74_Confidence_0.127_Length_2941 Locus 1930 Transcript $47 / 74$ Confidence 0.127 Length 2941 Locus_1956_Transcript_14/26_Confidence_0.583_Length_1507 Locus_1956_Transcript_14/26_Confidence_0.583_Length_1507 Locus_1964_Transcript_30/53_Confidence_0.329_Length_1309 Locus_1964_Transcript_30/53_Confidence_0.329_Length_1309 Locus_1964_Transcript_30/53_Confidence_0.329_Length_1309 Locus_1964_Transcript_30/53_Confidence_0.329_Length_1309 Locus_1991_Transcript_29/47_Confidence_0.347_Length 4472 Locus_1991_Transcript_29/47_Confidence_0.347_Length_4472 Locus_2003_Transcript_7/9_Confidence_0.621_Length_1227 Locus_2003_Transcript 7/9 Confidence 0.621 Length 1227

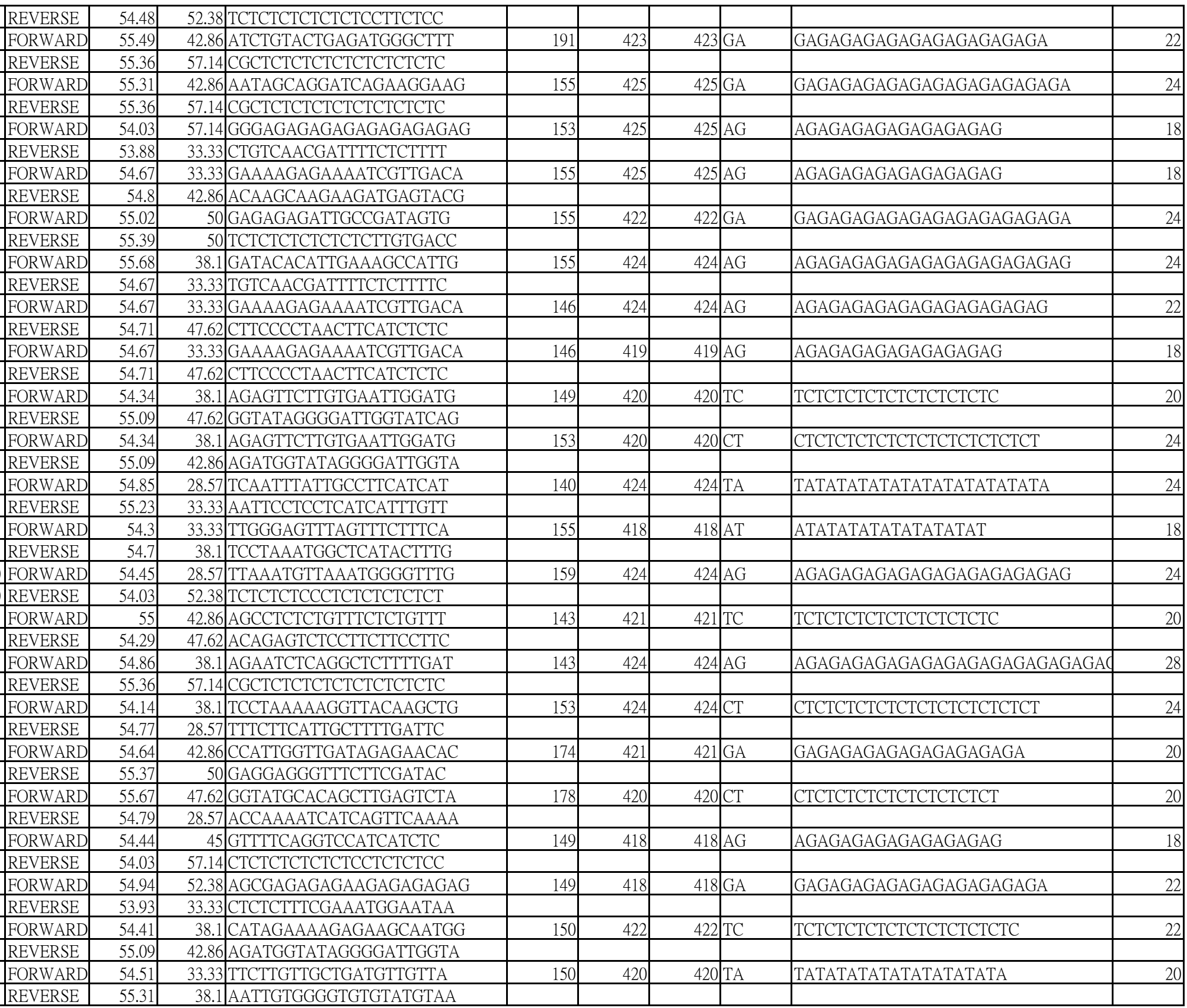




\begin{tabular}{|c|c|c|c|c|c|c|c|c|c|}
\hline Locus_2012_Transcript_51/68_Confidence_0.562_Length_2744 & FORWARD & 55.49 & \begin{tabular}{l|l}
40 & AAGCAAAGCTGGCTTATACA \\
\end{tabular} & 194 & 420 & 420 & CT & СТСТСТСТСТСТСТСТСТСТ & 20 \\
\hline Locus_2012_Transcript_51/68_Confidence_0.562_Length_2744 & REVERSE & 55.68 & \begin{tabular}{l|l|}
38.1 & GCTCGCAAATTTAACTCTCTT \\
\end{tabular} & & & & & & \\
\hline Locus_2035_Transcript_4/7_Confidence_0.600_Length_2778 & FORWARD & 55.76 & \begin{tabular}{l|l}
38.1 & GTTGCAATTATTCCACTGTTG \\
\end{tabular} & 155 & 424 & 424 & $\mathrm{CT}$ & СТСТСТСТСТСТСТСТСТСТСТСТ & 24 \\
\hline Locus_2035_Transcript_4/7_Confidence_0.600_Length_2778 & REVERSE & 54.99 & \begin{tabular}{l|l|} 
47.62 & CATGAGAGACTGAAGAAGGTG \\
\end{tabular} & & & & & & \\
\hline Locus_2036_Transcript_23/34_Confidence_0.563_Length_2233 & FORWARD & 54.81 & 38.1 TTTGTCCTTGTACCACTTGTT & 151 & 425 & 425 & AT & ATATATATATATATATATATATAT & 24 \\
\hline Locus_2036_Transcript_23/34_Confidence_0.563_Length_2233 & REVERSE & 54.88 & \begin{tabular}{l|l|}
31.82 & GGTGGATCAGTTAAATTTTCAT \\
\end{tabular} & & & & & & \\
\hline Locus_2068_Transcript_23/61_Confidence_0.418_Length_1152 & FORWARD & 55.07 & 36.36 GCAAATTATTGAAGGAAGAGAG & 162 & 419 & 419 & GA & GAGAGAGAGAGAGAGAGAGAGA & 22 \\
\hline Locus_2068_Transcript_23/61_Confidence_0.418_Length_1152 & REVERSE & 55.1 & 33.33 TCCTAGTATTCCAAATGCAAA & & & & & & \\
\hline Locus_2068_Transcript_23/61_Confidence_0.418_Length_1152 & FORWARD & 54.81 & 42.86 ATTGAAGGAAGAGAGAGGAGA & 155 & 419 & 419 & $\mathrm{AG}$ & AGAGAGAGAGAGAGAGAG & 18 \\
\hline Locus_2068_Transcript_23/61_Confidence_0.418_Length_1152 & REVERSE & 55.1 & 33.33 TCCTAGTATTCCAAATGCAAA & & & & & & \\
\hline Locus_2109_Transcript_33/44_Confidence_0.581_Length_3294 & FORWARD & 55.09 & 33.33 AATCCAATCCTTATCAAAAGC & 150 & 418 & 418 & CT & СТСТСТСТСТСТСТСТСТ & 18 \\
\hline Locus_2109_Transcript_33/44_Confidence_0.581_Length_3294 & REVERSE & 54.88 & 47.62 CCTGGGAAATTAGAGAGAGAG & & & & & & \\
\hline Locus_2109_Transcript_33/44_Confidence_0.581_Length_3294 & FORWARD & 55.67 & 38.1 TAATTTCCCAGGAACAATACC & 169 & 418 & 418 & CT & СТСТСТСТСТСТСТСТСТ & 18 \\
\hline Locus_2109_Transcript_33/44_Confidence_0.581_Length_3294 & REVERSE & 55.05 & 38.1 & & & & & & \\
\hline Locus_2116_Transcript_18/31_Confidence_0.597_Length_2082 & FORWARD & 55.41 & 28.57 TGCCTTATTAAATGAGCAAAA & 151 & 421 & 421 & CT & СТСТСТСТСТСТСТСТСТСТ & 20 \\
\hline Locus_2116_Transcript_18/31_Confidence_0.597_Length_2082 & REVERSE & 55.14 & 55 2 CTCTCTGTTCTCCACTCTCG & & & & & & \\
\hline Locus_2200_Transcript_12/21_Confidence_0.599_Length_6392 & FORWARD & 54.93 & 35 ACATATTCGGCGGTATAAAA & 141 & 418 & 418 & $\mathrm{TC}$ & TCTCТСТСТСТСТСТСТС & 18 \\
\hline Locus_2200_Transcript_12/21_Confidence_0.599_Length_6392 & REVERSE & 55.54 & 42.86 AAGGAATGTAAGAGCGAAGAG & & & & & & \\
\hline Locus_2246_Transcript_54/74_Confidence_0.464_Length_9675 & FORWARD & 55.1 & 27.27 TGCATTAGTTTGAAATGTGTTT & 149 & 424 & 424 & $\mathrm{CT}$ & СТСТСТСТСТСТСТСТСТСТСТСТ & 24 \\
\hline Locus_2246_Transcript_54/74_Confidence_0.464_Length_9675 & REVERSE & 55.09 & \begin{tabular}{l|l} 
42.86 & AGATGGTATAGGGGATTGGTA \\
\end{tabular} & & & & & & \\
\hline Locus_2263_Transcript_151/275_Confidence_0.095_Length_5794 & FORWARD & 54.14 & \begin{tabular}{l|l|}
38.1 & TCCTAAAAAGGTTACAAGCTG \\
\end{tabular} & 161 & 424 & 424 & $\mathrm{CT}$ & СТСТСТСТСТСТСТСТСТСТСТСТ & 24 \\
\hline Locus_2263_Transcript_151/275_Confidence_0.095_Length_5794 & REVERSE & 55.74 & \begin{tabular}{l|l|}
33.33 & CCCCAAATTACAAATCAAAGT \\
\end{tabular} & & & & & & \\
\hline Locus_2285_Transcript_1/6_Confidence_0.735_Length_3431 & FORWARD & 55.18 & \begin{tabular}{l|l|l|}
47.62 & GGGAGTACCACCACTAAAATC \\
\end{tabular} & 151 & 419 & 419 & GA & GAGAGAGAGAGAGAGAGA & 18 \\
\hline Locus_2285_Transcript_1/6_Confidence_0.735_Length_3431 & REVERSE & 57.51 & \begin{tabular}{l|l|}
50 & CTTTGTCTCCTGGTTTCAGC \\
\end{tabular} & & & & & & \\
\hline Locus_2367_Transcript_4/16_Confidence_0.536_Length_3739 & FORWARD & 54.96 & \begin{tabular}{l|l|}
38.1 & TCTGTGCTTTTCTTAACCTTG \\
\end{tabular} & 135 & 419 & 419 & $\mathrm{TC}$ & TCTCTCTCTCTCTCTCTC & 18 \\
\hline Locus_2367_Transcript_4/16_Confidence_0.536_Length_3739 & REVERSE & 54.78 & \begin{tabular}{l|l}
35 & ATTTTTGACAGGCTGAATGT \\
\end{tabular} & & & & & & \\
\hline Locus_2428_Transcript_52/101_Confidence_0.350_Length_2863 & FORWARD & 55.33 & \begin{tabular}{l|l|l} 
42.86 & TCCCGTAGATAGAAAAAGAGG \\
\end{tabular} & 146 & 426 & 426 & GT & GTGTGTGTGTGTGTGTGTGTGTGTGT & 26 \\
\hline Locus_2428_Transcript_52/101_Confidence_0.350_Length_2863 & REVERSE & 54.64 & \begin{tabular}{l|l|}
47.62 & CTATCACTGCTATCTCCTCCA \\
\end{tabular} & & & & & & \\
\hline Locus_2545_Transcript_28/48_Confidence_0.590_Length_2456 & FORWARD & 55.15 & \begin{tabular}{l|l|}
38.1 & TCACTGGATTCAAATCGATAC \\
\end{tabular} & 153 & 419 & 419 & $\mathrm{AG}$ & AGAGAGAGAGAGAGAGAG & 18 \\
\hline Locus_2545_Transcript_28/48_Confidence_0.590_Length_2456 & REVERSE & 54 & \begin{tabular}{l|l|}
33.33 & GTTGCTCTGAAAAGGATTTTA \\
\end{tabular} & & & & & & \\
\hline Locus_2577_Transcript_16/31_Confidence_0.608_Length_3830 & FORWARD & 55.09 & \begin{tabular}{l|l|}
40 & CACCATTCGTCACCTAATTT \\
\end{tabular} & 212 & 424 & 424 & $\mathrm{TC}$ & TCTCTCTCTCTCTCTCTCTCTCTC & 24 \\
\hline Locus_2577_Transcript_16/31_Confidence_0.608_Length_3830 & REVERSE & 56.36 & \begin{tabular}{l|l|l|}
55 & CTACTCCTGCACTCAAGCAG \\
\end{tabular} & & & & & & \\
\hline Locus_2641_Transcript_39/55_Confidence_0.330_Length_3582 & FORWARD & 55.43 & \begin{tabular}{l|l|l}
42.86 & TACCATCTCGTACATCTTTGC \\
\end{tabular} & 142 & 424 & 424 & $\mathrm{CT}$ & СТСТСТСТСТСТСТСТСТСТСТСТ & 24 \\
\hline Locus_2641_Transcript_39/55_Confidence_0.330_Length_3582 & REVERSE & 54.47 & \begin{tabular}{l|l|}
42.86 & GATGGTATAGGGGATTGGTAT \\
\end{tabular} & & & & & & \\
\hline Locus_2641_Transcript_39/55_Confidence_0.330_Length_3582 & FORWARD & 55.5 & \begin{tabular}{l|l|l|}
47.62 & TAGAAAACCAGCTCCTCTCTC \\
\end{tabular} & 153 & 424 & 424 & $\mathrm{CT}$ & СТСТСТСТСТСТСТСТСТСТСТСТ & 24 \\
\hline Locus_2641_Transcript_39/55_Confidence_0.330_Length_3582 & REVERSE & 55.09 & \begin{tabular}{l|l|} 
42.86 & AGATGGTATAGGGGATTGGTA \\
\end{tabular} & & & & & & \\
\hline Locus_2641_Transcript_39/55_Confidence_0.330_Length_3582 & FORWARD & 55.01 & \begin{tabular}{l|l}
50 & TACAGTCCCTGAATGGAGAC \\
\end{tabular} & 147 & 424 & 424 & $\mathrm{CT}$ & СТСТСТСТСТСТСТСТСТСТСТСТ & 24 \\
\hline Locus_2641_Transcript_39/55_Confidence_0.330_Length_3582 & REVERSE & 55.63 & 33.33 TGTAAGAGCGAAAGCAAATTA & & & & & & \\
\hline Locus_2685_Transcript_3/22_Confidence_0.679_Length_2573 & FORWARD & 53.11 & \begin{tabular}{l|l|}
38.1 & ATAAGCAGAGGATTGAGTCAT \\
\end{tabular} & 155 & 427 & 427 & $\mathrm{TA}$ & TATATATATATATATATATATATA & 24 \\
\hline Locus_2685_Transcript_3/22_Confidence_0.679_Length_2573 & REVERSE & 55.16 & 42.86 GCAGGTATGTCTGTTTGATGT & & & & & & \\
\hline Locus_2685_Transcript_3/22_Confidence_0.679_Length_2573 & FORWARD & 55.34 & 42.86 ATCAAACAGACATACCTGCTG & 170 & 427 & 427 & AT & ATATATATATATATATATATATATAT & 26 \\
\hline Locus_2685_Transcript_3/22_Confidence_0.679_Length_2573 & REVERSE & 54.96 & \begin{tabular}{l|l}
38.1 & GGCATGTCGTTTATTGATTAG \\
\end{tabular} & & & & & & \\
\hline Locus_2694_Transcript_7/20_Confidence_0.541_Length_2193 & FORWARD & 55.09 & 35 AACTTTGCTTCTCAAATCCA & 135 & 424 & 424 & CT & СТCTCTCTCTCTCTCTCTCTCTCT & 24 \\
\hline Locus_2694_Transcript_7/20_Confidence_0.541_Length_2193 & REVERSE & 54.83 & \begin{tabular}{l|l}
38.1 & AAAAGATGGTATAGGGGATTG \\
\end{tabular} & & & & & & \\
\hline Locus_2694_Transcript_7/20_Confidence_0.541_Length_2193 & FORWARD & 55.45 & \begin{tabular}{|l|l|}
52.38 & CAGCCTCTCCTACAACTCTCT \\
\end{tabular} & 143 & 424 & 424 & CT & СТСТСТСТСТСТСТСТСТСТСТСТ & 24 \\
\hline
\end{tabular}




\begin{tabular}{|c|c|c|c|c|c|c|c|c|c|c|}
\hline Locus_2694_Transcript_7/20_Confidence_0.541_Length_2193 & REVERSE & 55.5 & 47.62 & GAGAGAGGAGCTGGTTTTCTA & & & & & & \\
\hline Locus_2715_Transcript_25/35_Confidence_0.500_Length_4014 & FORWARD & 55.37 & 33.33 & TTGGAAACAGAAATCTGAATG & 179 & 421 & 421 & $\mathrm{AG}$ & AGAGAGAGAGAGAGAGAGAG & 20 \\
\hline Locus_2715_Transcript_25/35_Confidence_0.500_Length_4014 & REVERSE & 54.79 & 42.86 & AGAGGGTGAGGAAGAAGTAAA & & & & & & \\
\hline Locus_2725_Transcript_4/12_Confidence_0.599_Length_2797 & FORWARD & 54.94 & 42.86 & ATATTCCCTATGCTCCCTATG & 156 & 424 & 424 & $\mathrm{CT}$ & СТСТСТСТСТСТСТСТСТСТСТСТ & 24 \\
\hline Locus_2725_Transcript_4/12_Confidence_0.599_Length_2797 & REVERSE & 55.26 & 38.1 & GAGCAGAAAATGGAAAGAGAT & & & & & & \\
\hline Locus_2725_Transcript_4/12_Confidence_0.599_Length_2797 & FORWARD & 55.26 & 38.1 & ATCTCTTTCCATTTTCTGCTC & 150 & 424 & 424 & CT & СТСТСТСТСТСТСТСТСТСТ & 20 \\
\hline Locus_2725_Transcript_4/12_Confidence_0.599_Length_2797 & REVERSE & 55.07 & 40 & ATCAAGCTCTCCAAAGTTCA & & & & & & \\
\hline Locus_2755_Transcript_52/65_Confidence_0.583_Length_2972 & FORWARD & 55.04 & 42.86 & TGACAGGAGAGAGAAATCAGA & 151 & 423 & 423 & CT & СТСТСТСТСТСТСТСТСТСТСТ & 22 \\
\hline Locus_2755_Transcript_52/65_Confidence_0.583_Length_2972 & REVERSE & 55.24 & 28.57 & AAATAGAAAGCGAAAGCAAAT & & & & & & \\
\hline Locus_2767_Transcript_36/42_Confidence_0.591_Length_3879 & FORWARD & 54.87 & 38.1 & TCAGCTCCTCCCTTTATTTAT & 163 & 419 & 419 & TA & TATATATATATATATATA & 18 \\
\hline Locus_2767_Transcript_36/42_Confidence_0.591_Length_3879 & REVERSE & 55.58 & 47.62 & GAGGACCTCACTGAATACCAT & & & & & & \\
\hline Locus_2837_Transcript_9/73_Confidence_0.422_Length_4896 & FORWARD & 54.5 & 33.33 & TATACAACGAACAGCAATGAA & 154 & 420 & 420 & $\mathrm{AG}$ & AGAGAGAGAGAGAGAGAGAG & 20 \\
\hline Locus_2837_Transcript_9/73_Confidence_0.422_Length_4896 & REVERSE & 54.51 & 38.1 & TATATGCCAACCGTATTTCTC & & & & & & \\
\hline Locus_2862_Transcript_47/68_Confidence_0.389_Length_2908 & FORWARD & 56.32 & 45 & ACCTTTCTCCCGATACAGAA & 145 & 420 & 420 & $\mathrm{AG}$ & AGAGAGAGAGAGAGAGAGAG & 20 \\
\hline Locus_2862_Transcript_47/68_Confidence_0.389_Length_2908 & REVERSE & 55 & 42.86 & ТTСТССТТССТТСТССТСТТА & & & & & & \\
\hline Locus_2888_Transcript_3/41_Confidence_0.517_Length_1679 & FORWARD & 54.9 & 31.82 & AAGCCATATCTCCAGAAAAATA & 145 & 420 & 420 & $\mathrm{CT}$ & СТСТСТСТСТСТСТСТСТСТ & 20 \\
\hline Locus_2888_Transcript_3/41_Confidence_0.517_Length_1679 & REVERSE & 55.23 & 38.1 & GCCTTTTGTAAATCCTCTTGT & & & & & & \\
\hline Locus_2951_Transcript_8/19_Confidence_0.597_Length_2512 & FORWARD & 55.48 & 42.86 & TAAGAATTGAGGAAGCTAGGG & 143 & 420 & 420 & GA & GAGAGAGAGAGAGAGAGAGA & 20 \\
\hline Locus_2951_Transcript_8/19_Confidence_0.597_Length_2512 & REVERSE & 54.99 & 38.1 & AAACGCTTAAACCATCTCTCT & & & & & & \\
\hline Locus_2979_Transcript_2/13_Confidence_0.689_Length_1583 & FORWARD & 55.02 & 57.14 & CCGACTCTCTCTCTCTCTCTC & 149 & 419 & 419 & $\mathrm{TC}$ & TCTCTCTCTCTCTCTCTC & 18 \\
\hline Locus_2979_Transcript_2/13_Confidence_0.689_Length_1583 & REVERSE & 54.38 & 38.1 & ATTAAATCTTGCTAGCTGCTG & & & & & & \\
\hline Locus_2982_Transcript_17/50_Confidence_0.248_Length_1021 & FORWARD & 54.97 & 33.33 & TTGAATCCAAAGTTGTCCTTA & 99 & 419 & 419 & GA & GAGAGAGAGAGAGAGAGA & 18 \\
\hline Locus_2982_Transcript_17/50_Confidence_0.248_Length_1021 & REVERSE & 56.62 & 33.33 & CAAAGACCAATTTTGAAAAGC & & & & & & \\
\hline Locus_2993_Transcript_10/23_Confidence_0.690_Length_1442 & FORWARD & 54.76 & 38.1 & GAGTTGGAGGGAATTAAAAAG & 165 & 424 & 424 & GA & GAGAGAGAGAGAGAGAGAGAGAGA & 24 \\
\hline Locus_2993_Transcript_10/23_Confidence_0.690_Length_1442 & REVERSE & 55.36 & 57.14 & CGCTCTCTCTCTCTCTCTCTC & & & & & & \\
\hline Locus_2993_Transcript_10/23_Confidence_0.690_Length_1442 & FORWARD & 55.53 & 47.62 & GAGGAGCAAAGCTAGATCACT & 179 & 424 & 424 & $\mathrm{AG}$ & AGAGAGAGAGAGAGAGAG & 18 \\
\hline Locus_2993_Transcript_10/23_Confidence_0.690_Length_1442 & REVERSE & 53.88 & 33.33 & CTGTCAACGATTTTCTCTTTT & & & & & & \\
\hline Locus_2993_Transcript_10/23_Confidence_0.690_Length_1442 & FORWARD & 54.67 & 33.33 & GAAAAGAGAAAATCGTTGACA & 143 & 424 & 424 & $\mathrm{AG}$ & AGAGAGAGAGAGAGAGAGAGAG & 22 \\
\hline Locus_2993_Transcript_10/23_Confidence_0.690_Length_1442 & REVERSE & 56.01 & 47.62 & CGAACCTCGACCTGTAATACT & & & & & & \\
\hline Locus_2993_Transcript_10/23_Confidence_0.690_Length_1442 & FORWARD & 55.53 & 47.62 & GAGGAGCAAAGCTAGATCACT & 155 & 422 & 422 & $\mathrm{AG}$ & AGAGAGAGAGAGAGAGAGAGAGAG & 24 \\
\hline Locus_2993_Transcript_10/23_Confidence_0.690_Length_1442 & REVERSE & 55.39 & 50 & TCTCTCTCTCTCTCTTGTGACC & & & & & & \\
\hline Locus_3019_Transcript_9/140_Confidence_0.258_Length_6728 & FORWARD & 55.26 & 47.62 & GAGAAGGAACGGATAGAGAGA & 148 & 422 & 422 & $\mathrm{CT}$ & СТСТСТСТСТСТСТСТСТСТСТ & 22 \\
\hline Locus_3019_Transcript_9/140_Confidence_0.258_Length_6728 & REVERSE & 54.61 & 47.62 & CAGAAGCACAGAGAGAAAGAG & & & & & & \\
\hline Locus_3066_Transcript_35/44_Confidence_0.511_Length_2504 & FORWARD & 55.04 & 47.62 & TCTCGTTCTCTCTCTCTCCTT & 156 & 420 & 420 & $\mathrm{CT}$ & СТСТСТСТСТСТСТСТСТСТ & 20 \\
\hline Locus_3066_Transcript_35/44_Confidence_0.511_Length_2504 & REVERSE & 55.5 & 47.62 & GAGAGAGGAGCTGGTTTTCTA & & & & & & \\
\hline Locus_3066_Transcript_35/44_Confidence_0.511_LEngth_2504 & FORWARD & 54.85 & 38.1 & CGCTCTTACATTCCTTCAATA & 142 & 424 & 424 & CT & СТСТСТСТСТСТСТСТСТСТ & 20 \\
\hline Locus_3066_Transcript_35/44_Confidence_0.511_Length_2504 & REVERSE & 55.19 & 57.14 & GGCAGAGAGAGAGAGAGAGAG & & & & & & \\
\hline Locus_3066_Transcript_35/44_Confidence_0.511_Length_2504 & FORWARD & 55.45 & 31.82 & TCCTTCAATAATTTGCTTTCTC & 158 & 424 & 424 & $\mathrm{CT}$ & СТСТСТСТСТСТСТСТСТСТСТСТ & 24 \\
\hline Locus_3066_Transcript_35/44_Confidence_0.511_Length_2504 & REVERSE & 58.3 & 55 & AGGGCTGATATGGAGGAGAG & & & & & & \\
\hline Locus_3066_Transcript_35/44_Confidence_0.511_Length_2504 & FORWARD & 54.14 & 38.1 & TCCTAAAAAGGTTACAAGCTG & 148 & 420 & 420 & CT & СТСТСТСТСТСТСТСТСТСТСТСТ & 24 \\
\hline Locus_3066_Transcript_35/44_Confidence_0.511_Length_2504 & REVERSE & 55.63 & 52.38 & GCCAGAGAGAGAGAGAGAGAA & & & & & & \\
\hline Locus_3066_Transcript_35/44_Confidence_0.511_Length_2504 & FORWARD & 56 & 57.14 & ССССТСТСТСТСТСТСТСТСТ & 135 & 420 & 420 & CT & СТСТСТСТСТСТСТСТСТСТСТСТ & 24 \\
\hline Locus_3066_Transcript_35/44_Confidence_0.511_Length_2504 & REVERSE & 55.03 & 47.62 & AAGTAGAAGGTGTACCCAAGG & & & & & & \\
\hline Locus_3129_Transcript_23/61_Confidence_0.364_Length_1390 & FORWARD & 55.12 & 33.33 & AGCATGAGGCAATTACAAATA & 171 & 421 & 421 & AT & ATATATATATATATATATATATATAT & 26 \\
\hline Locus_3129_Transcript_23/61_Confidence_0.364_Length_1390 & REVERSE & 54.85 & 42.86 & TATCACATATGACCCCTGTTC & & & & & & \\
\hline
\end{tabular}




\begin{tabular}{|c|c|c|c|c|c|c|c|c|c|}
\hline Locus_3129_Transcript_23/61_Confidence_0.364_Length_1390 & FORWARD & 55.11 & \begin{tabular}{l|l}
33.33 & GAGATTGAATTTCATGTTGGA \\
\end{tabular} & 145 & 421 & 421 & TA & TATATATATATATATATATA & 20 \\
\hline Locus_3129_Transcript_23/61_Confidence_0.364_Length_1390 & REVERSE & 55.53 & \begin{tabular}{l|l|}
33.33 & CATTGCACACATTTTATCACA \\
\end{tabular} & & & & & & \\
\hline Locus_3217_Transcript_10/34_Confidence_0.511_Length_2127 & FORWARD & 55.23 & \begin{tabular}{l|l}
47.62 & TTCTACCGTCTTCTACCTTCC \\
\end{tabular} & 155 & 423 & 423 & $\mathrm{TC}$ & TCTCTCTCTCТСТСТСТСТСТС & 22 \\
\hline Locus_3217_Transcript_10/34_Confidence_0.511_Length_2127 & REVERSE & 55.01 & \begin{tabular}{l|l} 
40.91 & AAGGTAGTCCTTGAGCATTAGA \\
\end{tabular} & & & & & & \\
\hline Locus_3250_Transcript_12/26_Confidence_0.673_Length_2536 & FORWARD & 54.73 & \begin{tabular}{l|l|} 
40 & CTTACTTCACCTTTCGCAAT \\
\end{tabular} & 146 & 422 & 422 & $\mathrm{TC}$ & TСТСТСТСТСТСТСТСТСТСТС & 22 \\
\hline Locus_3250_Transcript_12/26_Confidence_0.673_Length_2536 & REVERSE & 55.09 & \begin{tabular}{l|l} 
42.86 & AGATGGTATAGGGGATTGGTA \\
\end{tabular} & & & & & & \\
\hline Locus_3278_Transcript_15/23_Confidence_0.595_Length_3630 & FORWARD & 55.04 & 42.86 TGGACAGAAGCAACAGATACT & 152 & 418 & 418 & CT & СТСТСТСТСТСТСТСТСТ & 18 \\
\hline Locus_3278_Transcript_15/23_Confidence_0.595_Length_3630 & REVERSE & 55.08 & \begin{tabular}{l|l}
38.1 & AGTACAATTTTCTTCCCGAAC \\
\end{tabular} & & & & & & \\
\hline Locus_3323_Transcript_8/85_Confidence_0.539_Length_2616 & FORWARD & 53.96 & \begin{tabular}{l|l|}
28.57 & GAAAGCAAATTATTGAAGGAA \\
\end{tabular} & 151 & 423 & 423 & GA & GAGAGAGAGAGAGAGAGAGAGA & 22 \\
\hline Locus_3323_Transcript_8/85_Confidence_0.539_Length_2616 & REVERSE & 55.14 & 52.38 CCGTTAGCTTACTCTCCTCTC & & & & & & \\
\hline Locus_3323_Transcript_8/85_Confidence_0.539_Length_2616 & FORWARD & 54.6 & 52.38 AGAGAGAGAGGAGAGAACTCG & 153 & 423 & 423 & $\mathrm{AG}$ & AGAGAGAGAGAGAGAGAG & 18 \\
\hline Locus_3323_Transcript_8/85_Confidence_0.539_Length_2616 & REVERSE & 55.41 & \begin{tabular}{l|l|} 
42.86 & AACAAGGAGTTACGTCCAGAT \\
\end{tabular} & & & & & & \\
\hline Locus_3349_Transcript_10/12_Confidence_0.618_Length_1410 & FORWARD & 54.79 & \begin{tabular}{l|l|}
42.86 & GCCTGATTGTGTCATAAACTC \\
\end{tabular} & 135 & 423 & 423 & TA & TATATATATATATATATATATA & 22 \\
\hline Locus_3349_Transcript_10/12_Confidence_0.618_Length_1410 & REVERSE & 54.3 & \begin{tabular}{l|l|}
38.1 & TAATCGACAGACAACAAGTGA \\
\end{tabular} & & & & & & \\
\hline Locus_3366_Transcript_8/31_Confidence_0.577_Length_4065 & FORWARD & 55.73 & \begin{tabular}{l|l|l|}
33.33 & TGATGCTGAAGAATGATTGAT \\
\end{tabular} & 152 & 419 & 419 & TA & TATATATATATATATATA & 18 \\
\hline Locus_3366_Transcript_8/31_Confidence_0.577_Length_4065 & REVERSE & 54.91 & \begin{tabular}{l|l|l|}
33.33 & TCATCAGGTTCTATGCAAAAT \\
\end{tabular} & & & & & & \\
\hline Locus_3376_Transcript_101/115_Confidence_0.336_Length_5494 & FORWARD & 56.01 & \begin{tabular}{l|l|l}
42.86 & GATATGGAGGATGAATGGAAG \\
\end{tabular} & 150 & 422 & 422 & GA & GAGAGAGAGAGAGAGAGAGAGA & 22 \\
\hline Locus_3376_Transcript_101/115_Confidence_0.336_Length_5494 & REVERSE & 55.44 & \begin{tabular}{l|l|} 
52.38 & ACACTGTCTCTCCACCTCTCT \\
\end{tabular} & & & & & & \\
\hline Locus_3376_Transcript_101/115_Confidence_0.336_Length_5494 & FORWARD & 55.37 & \begin{tabular}{l|l} 
47.62 & AGAGAGAGAGAAGAGCTCGAA \\
\end{tabular} & 153 & 422 & 422 & $\mathrm{AG}$ & AGAGAGAGAGAGAGAGAGAGAG & 22 \\
\hline Locus_3376_Transcript_101/115_Confidence_0.336_Length_5494 & REVERSE & 54.98 & \begin{tabular}{l|l|}
52.38 & CAGGTTTCTCTCTACCTAGCC \\
\end{tabular} & & & & & & \\
\hline Locus_3377_Transcript_36/83_Confidence_0.389_Length_4097 & FORWARD & 55.27 & \begin{tabular}{l|l|} 
42.86 & CTAGCTGATTATGCCAAGATG \\
\end{tabular} & 154 & 423 & 423 & GA & GAGAGAGAGAGAGAG & 22 \\
\hline Locus_3377_Transcript_36/83_Confidence_0.389_Length_4097 & REVERSE & 54.67 & \begin{tabular}{l|l|l}
33.33 & TGTCAACGATTTTCTCTTTTC \\
\end{tabular} & & & & & & \\
\hline Locus_3377_Transcript_36/83_Confidence_0.389_Length_4097 & FORWARD & 54.48 & \begin{tabular}{l|l}
52.38 & GGAGAAGGAGAGAGAGAGAGA \\
\end{tabular} & 166 & 423 & 423 & $\mathrm{AG}$ & AGAGAGAG & 22 \\
\hline Locus_3377_Transcript_36/83_Confidence_0.389_Length_4097 & REVERSE & 55.98 & \begin{tabular}{l|l|}
42.86 & GGACTTAGGAGGCAATGAATA \\
\end{tabular} & & & & & & \\
\hline Locus_3377_Transcript_36/83_Confidence_0.389_Length_4097 & FORWARD & 54.67 & \begin{tabular}{l|l|}
33.33 & GAAAAGAGAAAATCGTTGACA \\
\end{tabular} & 169 & 424 & 424 & $\mathrm{AG}$ & AGAGAGAGAGAGAGAGAGAGAG & 22 \\
\hline Locus_3377_Transcript_36/83_Confidence_0.389_Length_4097 & REVERSE & 54.63 & \begin{tabular}{l|l} 
42.86 & AGCACCAATGAAGACTGTTAG \\
\end{tabular} & & & & & & \\
\hline Locus_3386_Transcript_60/69_Confidence_0.579_Length_3950 & FORWARD & 55.06 & \begin{tabular}{l|l}
38.1 & CCGTATTAAAATCAACCTCCT \\
\end{tabular} & 147 & 424 & 424 & TA & TATATATATATATATATATATATA & 24 \\
\hline Locus_3386_Transcript_60/69_Confidence_0.579_Length_3950 & REVERSE & 54.86 & \begin{tabular}{l|l|l}
38.1 & TGATGAGGAAGAAGAATACCA \\
\end{tabular} & & & & & & \\
\hline Locus_3429_Transcript_28/38_Confidence_0.582_Length_2249 & FORWARD & 55.09 & \begin{tabular}{l|l} 
42.86 & AGATGGTATAGGGGATTGGTA \\
\end{tabular} & 158 & 419 & 419 & $\mathrm{AG}$ & AGAGAGAGAGAGAGAGAG & 18 \\
\hline Locus_3429_Transcript_28/38_Confidence_0.582_Length_2249 & REVERSE & 54.98 & \begin{tabular}{l|l|}
50 & ACAGAGGTTTCCTTCTCCTC \\
\end{tabular} & & & & & & \\
\hline Locus_3493_Transcript_10/41_Confidence_0.314_Length_1674 & FORWARD & 55.24 & 47.62 CACAGACAAAGCAGAAGAGTC & 165 & 421 & 421 & GA & GAGAGAGAGAGAGAGAGAGA & 20 \\
\hline Locus_3493_Transcript_10/41_Confidence_0.314_Length_1674 & REVERSE & 54.7 & \begin{tabular}{l|l|}
42.86 & GCTGTTGCATCTCAATAGTCT \\
\end{tabular} & & & & & & \\
\hline Locus_3493_Transcript_10/41_Confidence_0.314_Length_1674 & FORWARD & 55.03 & 57.14 CTGGAGAGGAGAGAGAGAGAG & 140 & 421 & 421 & GA & GAGAGAGAGAGAGAGAGAGAGAGA & 24 \\
\hline Locus_3493_Transcript_10/41_Confidence_0.314_Length_1674 & REVERSE & 54.7 & \begin{tabular}{l|l} 
42.86 & AGCTGTTGCATCTCAATAGTC \\
\end{tabular} & & & & & & \\
\hline Locus_3503_Transcript_23/26_Confidence_0.571_Length_1262 & FORWARD & 54.9 & \begin{tabular}{l|l|} 
42.86 & CATTTTACAACAGAGGGACAG \\
\end{tabular} & 227 & 419 & 419 & GA & GAGAGAGAGAGAGAGAGA & 18 \\
\hline Locus_3503_Transcript_23/26_Confidence_0.571_Length_1262 & REVERSE & 55.84 & \begin{tabular}{l|l|}
45 & AATGAGCTCGACCACATCTA \\
\end{tabular} & & & & & & \\
\hline Locus_3514_Transcript_7/31_Confidence_0.588_Length_2145 & FORWARD & 54.95 & \begin{tabular}{l|l|}
50 & GTATGGTGTGGAGTTCTCGT \\
\end{tabular} & 155 & 424 & 424 & $\mathrm{TC}$ & TCTCTCТСТСТСТСТСТСТС & 20 \\
\hline Locus_3514_Transcript_7/31_Confidence_0.588_Length_2145 & REVERSE & 55.2 & \begin{tabular}{l|l|} 
42.86 & ATAGGGGATTGGTATCAGAGA \\
\end{tabular} & & & & & & \\
\hline Locus_3514_Transcript_7/31_Confidence_0.588_Length_2145 & FORWARD & 54.5 & \begin{tabular}{|l|l|}
52.38 & GCCATCTCTCTCTCTCTCTCT \\
\end{tabular} & 162 & 424 & 424 & CT & СТСТСТСТСТСТСТСТСТСТСТСТ & 24 \\
\hline Locus_3514_Transcript_7/31_Confidence_0.588_Length_2145 & REVERSE & 54.57 & \begin{tabular}{l|l|} 
42.86 & CGACTAGGGTTTTCTTCTTCT \\
\end{tabular} & & & & & & \\
\hline Locus_3552_Transcript_133/133_Confidence_0.495_Length_4716 & FORWARD & 54.91 & \begin{tabular}{l|l|}
33.33 & AAGCATGAAATCCTCAATACA \\
\end{tabular} & 146 & 421 & 421 & $\mathrm{AG}$ & AGAGAGAGAGAGAGAGAGAG & 20 \\
\hline Locus_3552_Transcript_133/133_Confidence_0.495_Length_4716 & REVERSE & 55.68 & \begin{tabular}{l|l|}
38.1 & AGCTTGGTGGCATATAGTTTT \\
\end{tabular} & & & & & & \\
\hline Locus_3567_Transcript_11/41_Confidence_0.437_Length_1143 & FORWARD & 55.18 & \begin{tabular}{|l|l|}
33.33 & AAATTTCTAGGGTGAATTTCG \\
\end{tabular} & 142 & 424 & 424 & GA & GAGAGAGAGAGAGAGAGAGAGAGA & 24 \\
\hline Locus_3567_Transcript_11/41_Confidence_0.437_Length_1143 & REVERSE & 55.36 & \begin{tabular}{ll|l} 
57.14 & CGCTCTCTCTCTCTCTCTCTC \\
\end{tabular} & & & & & & \\
\hline Locus_3567_Transcript_11/41_Confidence_0.437_Length_1143 & FORWARD & 54.52 & \begin{tabular}{l|l|l|} 
42.86AGTAGAACCTTGAATCTGA \\
\end{tabular} & 145 & 424 & 424 & $\mathrm{AG}$ & AGAGAGAGAGAGAGAGAGAGAGAG & 24 \\
\hline
\end{tabular}




\begin{tabular}{|c|c|c|c|c|c|c|c|c|c|c|}
\hline Locus_3567_Transcript_11/41_Confidence_0.437_Length_1143 & REVERSE & 55.39 & 50 & TCTCTCTCTCTCTCTTGTGACC & & & & & & \\
\hline Locus_3567_Transcript_11/41_Confidence_0.437_Length_1143 & FORWARD & 54.77 & 52.17 & AGAGAGAGAGAGAGAGAGAGAGC & 167 & 424 & 424 & $\mathrm{AG}$ & AGAGAGAGAGAGAGAGAG & 18 \\
\hline Locus_3567_Transcript_11/41_Confidence_0.437_Length_1143 & REVERSE & 55.05 & 38.1 & TTGTTGCCACTTGACTATTCT & & & & & & \\
\hline Locus_3599_Transcript_11/22_Confidence_0.635_Length_1716 & FORWARD & 55.58 & 42.86 & ACCAGAAATCCTAACACATCC & 163 & 427 & 427 & $\mathrm{AG}$ & AGAGAGAGAGAGAGAGAGAGAGAGAG & 26 \\
\hline Locus_3599_Transcript_11/22_Confidence_0.635_Length_1716 & REVERSE & 55.36 & 57.14 & CGCTCTCTCTCTCTCTCTCTC & & & & & & \\
\hline Locus_3599_Transcript_11/22_Confidence_0.635_Length_1716 & FORWARD & 54.59 & 38.1 & AAAAACTGTTGACGTACAAGG & 168 & 427 & 427 & $\mathrm{AG}$ & AGAGAGAGAGAGAGAGAGAGAGAG & 24 \\
\hline Locus_3599_Transcript_11/22_Confidence_0.635_Length_1716 & REVERSE & 54.67 & 33.33 & TGTCAACGATTTTCTCTTTTC & & & & & & \\
\hline Locus_3599_Transcript_11/22_Confidence_0.635_Length_1716 & FORWARD & 54.16 & 52.38 & CAGCAGAGAGAGAGAGAGAGA & 164 & 427 & 427 & $\mathrm{AG}$ & AGAGAGAGAGAGAGAGAG & 18 \\
\hline Locus_3599_Transcript_11/22_Confidence_0.635_Length_1716 & REVERSE & 55.31 & 38.1 & GCCAAATCTACGCCTATTTAT & & & & & & \\
\hline Locus_3599_Transcript_11/22_Confidence_0.635_Length_1716 & FORWARD & 54.94 & 55 & GTCGGTCACAAGAGAGAGAG & 151 & 427 & 427 & $\mathrm{AG}$ & AGAGAGAGAGAGAGAGAGAGAG & 22 \\
\hline Locus_3599_Transcript_11/22_Confidence_0.635_Length_1716 & REVERSE & 54.3 & 42.86 & CTGTCAACGATTTTCTCTAGG & & & & & & \\
\hline Locus_3599_Transcript_11/22_Confidence_0.635_Length_1716 & FORWARD & 54.84 & 38.1 & GGATTTGACCTCTTTGGTATT & 125 & 419 & 419 & $\mathrm{AG}$ & AGAGAGAGAGAGAGAGAGAGAG & 22 \\
\hline Locus_3599_Transcript_11/22_Confidence_0.635_Length_1716 & REVERSE & 55.31 & 38.1 & GCCAAATCTACGCCTATTTAT & & & & & & \\
\hline Locus_3599_Transcript_11/22_Confidence_0.635_Length_1716 & FORWARD & 55.11 & 38.1 & TCCTAGAGAAAATCGTTGACA & 146 & 422 & 422 & $\mathrm{AG}$ & AGAGAGAGAGAGAGAGAGAGAG & 22 \\
\hline Locus_3599_Transcript_11/22_Confidence_0.635_Length_1716 & REVERSE & 55.31 & 42.86 & TGAACTGAGCGAGTATTAGGA & & & & & & \\
\hline Locus_3647_Transcript_17/28_Confidence_0.605_Length_1346 & FORWARD & 54.45 & 42.86 & GCTGATTGACTGTCTTCAGAT & 157 & 421 & 421 & $\mathrm{TA}$ & TATATATATATATATATATA & 20 \\
\hline Locus_3647_Transcript_17/28_Confidence_0.605_Length_1346 & REVERSE & 55.02 & 35 & CGAAAATGTGCATAGCAATA & & & & & & \\
\hline Locus_3707_Transcript_29/31_Confidence_0.653_Length_4149 & FORWARD & 55.57 & 42.86 & ATCTAGTTGCAGTTTCCCACT & 158 & 418 & 418 & $\mathrm{AG}$ & AGAGAGAGAGAGAGAGAG & 18 \\
\hline Locus_3707_Transcript_29/31_Confidence_0.653_Length_4149 & REVERSE & 54.93 & 47.62 & TAAGTCGCTGCACCTATACTC & & & & & & \\
\hline Locus_3707_Transcript_29/31_Confidence_0.653_Length_4149 & FORWARD & 54.27 & 42.86 & ACGGAGTACATAAATCGACAC & 138 & 427 & 427 & $\mathrm{AG}$ & AGAGAGAGAGAGAGAGAGAGAGAGAG & 26 \\
\hline Locus_3707_Transcript_29/31_Confidence_0.653_Length_4149 & REVERSE & 55.36 & 57.14 & CGCTCTCTCTCTCTCTCTCTC & & & & & & \\
\hline Locus_3707_Transcript_29/31_Confidence_0.653_Length_4149 & FORWARD & 54.57 & 33.33 & TGAGCTTTAAAAAGCAAAGAG & 151 & 427 & 427 & $\mathrm{AG}$ & GAGAGAG & 24 \\
\hline Locus_3707_Transcript_29/31_Confidence_0.653_Length_4149 & REVERSE & 54.99 & 27.27 & TTGGGATAAGCTAATTTTTGTT & & & & & & \\
\hline Locus_3738_Transcript_17/26_Confidence_0.610_Length_2600 & FORWARD & 53.96 & 28.57 & GAAAGCAAATTATTGAAGGAA & 133 & 423 & 423 & GA & GAGAGAGAGAGAGAGAGAGAGA & 22 \\
\hline Locus_3738_Transcript_17/26_Confidence_0.610_Length_2600 & REVERSE & 54.67 & 33.33 & TGTCAACGATTTTCTCTTTTC & & & & & & \\
\hline Locus_3738_Transcript_17/26_Confidence_0.610_Length_2600 & FORWARD & 54.86 & 38.1 & TGAAGGAATGTAATGGAGAGA & 173 & 423 & 423 & $\mathrm{AG}$ & AGAGAGAGAGAGAGAGAGAGAGAG & 24 \\
\hline Locus_3738_Transcript_17/26_Confidence_0.610_Length_2600 & REVERSE & 55.31 & 38.1 & GCCAAATCTACGCCTATTTAT & & & & & & \\
\hline Locus_3738_Transcript_17/26_Confidence_0.610_Length_2600 & FORWARD & 54.67 & 33.33 & GAAAAGAGAAAATCGTTGACA & 145 & 424 & 424 & $\mathrm{AG}$ & AGAGAGAGAGAGAGAGAGAGAG & 22 \\
\hline Locus_3738_Transcript_17/26_Confidence_0.610_Length_2600 & REVERSE & 55.14 & 47.62 & AGGGAGATGTAGATCGAGAAG & & & & & & \\
\hline Locus_3751_Transcript_11/22_Confidence_0.652_Length_3704 & FORWARD & 55.06 & 42.86 & AATCACGGAGAAGTAGAAACC & 161 & 421 & 421 & CT & СТСТСТСТСТСТСТСТСТСТ & 20 \\
\hline Locus_3751_Transcript_11/22_Confidence_0.652_Length_3704 & REVERSE & 55.2 & 42.86 & ATAGGGGATTGGTATCAGAGA & & & & & & \\
\hline Locus_3804_Transcript_9/13_Confidence_0.714_Length_834 & FORWARD & 54.75 & 33.33 & TTAACTCCACCAACACAATTT & 158 & 422 & 422 & $\mathrm{AG}$ & AGAGAGAGAGAGAGAGAGAGAG & 22 \\
\hline Locus_3804_Transcript_9/13_Confidence_0.714_Length_834 & REVERSE & 55.2 & 38.1 & CTTCCCAAGTGATTCTTCTTT & & & & & & \\
\hline Locus_3833_Transcript_28/31_Confidence_0.563_Length_2262 & FORWARD & 55.24 & 50 & AAAGAGTAAGCCGTCCCTAC & 177 & 427 & 427 & GA & GAGAGAGAGAGAGAGAGAGAGAGAGA & 26 \\
\hline Locus_3833_Transcript_28/31_Confidence_0.563_Length_2262 & REVERSE & 55.36 & 57.14 & CGCTCTCTCTCTCTCTCTCTC & & & & & & \\
\hline Locus_3841_Transcript_11/17_Confidence_0.621_Length_1296 & FORWARD & 54.98 & 42.86 & GAAAGTTAACACCTGGGACTT & 150 & 420 & 420 & $\mathrm{AT}$ & ATATATATATATATATATAT & 20 \\
\hline Locus_3841_Transcript_11/17_Confidence_0.621_Length_1296 & REVERSE & 54.43 & 42.86 & ACTGCACATAAAGACCGATAC & & & & & & \\
\hline Locus_3884_Transcript_4/26_Confidence_0.601_Length_1985 & FORWARD & 55.45 & 42.86 & GTTTCTCTCTCGCTCGATATT & 152 & 421 & 421 & GA & GAGAGAGAGAGAGAGAGAGA & 20 \\
\hline Locus_3884_Transcript_4/26_Confidence_0.601_Length_1985 & REVERSE & 57.48 & 55 & CTCGCCTGTGATCTCTTACC & & & & & & \\
\hline Locus_3889_Transcript_15/25_Confidence_0.516_Length_1200 & FORWARD & 54.95 & 47.62 & GTGTTGACAAGGTAGAAGACG & 166 & 424 & 424 & CT & СТСТСТСТСТСТСТСТСТСТСТСТ & 24 \\
\hline Locus_3889_Transcript_15/25_Confidence_0.516_Length_1200 & REVERSE & 54.69 & 47.62 & TGTAAGAGCGAGAGAGAGAGA & & & & & & \\
\hline Locus_3912_Transcript_6/19_Confidence_0.588_Length_2575 & FORWARD & 55.2 & 47.62 & TCCCTGTTCTCACTGTGTTAC & 164 & 418 & 418 & $\mathrm{TC}$ & TCTCTCTCTCTCTCTCTC & 18 \\
\hline Locus_3912_Transcript_6/19_Confidence_0.588_Length_2575 & REVERSE & 54.86 & 33.33 & ACTTTCAAGGTTAAGCCATTT & & & & & & \\
\hline Locus_3913_Transcript_15/41_Confidence_0.601_Length_1484 & FORWARD & 55.08 & 42.86 & CAGTCAAACACCCACTAATGT & 151 & 424 & 424 & $\mathrm{TC}$ & TCTCTCTCTCTCTCTCTCTC & 20 \\
\hline Locus_3913_Transcript_15/41_Confidence_0.601_Length_1484 & REVERSE & 55.09 & 47.62 & GGTATAGGGGATTGGTATCAG & & & & & & \\
\hline
\end{tabular}




\begin{tabular}{|c|c|c|c|c|c|c|c|c|c|}
\hline Locus_3913_Transcript_15/41_Confidence_0.601_Length_1484 & FORWARD & 55.08 & \begin{tabular}{l|l|} 
42.86 & CAGTCAAACACCCACTAATGT \\
\end{tabular} & 155 & 424 & 424 & CT & СТСТСТСТСТСТСТСТСТСТСТСТ & 24 \\
\hline Locus_3913_Transcript_15/41_Confidence_0.601_Length_1484 & REVERSE & 55.09 & \begin{tabular}{l|l|} 
42.86 & AGATGGTATAGGGGATTGGTA \\
\end{tabular} & & & & & & \\
\hline Locus_3925_Transcript_14/26_Confidence_0.340_Length_1799 & FORWARD & 56.1 & \begin{tabular}{l|l|}
45 & GAAGGCAGTTCTGGTTTCTT \\
\end{tabular} & 152 & 424 & 424 & CT & СТСТСТСТСТСТСТСТСТСТСТСТ & 24 \\
\hline Locus_3925_Transcript_14/26_Confidence_0.340_Length_1799 & REVERSE & 55.5 & \begin{tabular}{l|l|}
47.62 & GAGAGAGGAGCTGGTTTTCTA \\
\end{tabular} & & & & & & \\
\hline Locus_3925_Transcript_14/26_Confidence_0.340_Length_1799 & FORWARD & 55.02 & 57.14 CGGACTCTCTCTCTCTCTCTC & 169 & 424 & 424 & CT & СТСТСТСТСТСТСТСТСТСТСТСТ & 24 \\
\hline Locus_3925_Transcript_14/26_Confidence_0.340_Length_1799 & REVERSE & 54.44 & 33.33 GTCGAAAGCAAATTATTGAAG & & & & & & \\
\hline Locus_3928_Transcript_117/135_Confidence_0.428_Length_6430 & FORWARD & 54.9 & 42.86 GATACTCAACTTTCGAGCGTA & 148 & 420 & 420 & CT & СТСТСТСТСТСТСТСТСТСТ & 20 \\
\hline Locus_3928_Transcript_117/135_Confidence_0.428_Length_6430 & REVERSE & 55.7 & \begin{tabular}{l|l|} 
42.86 & ATATTAGGAGTGGTTGGATGC \\
\end{tabular} & & & & & & \\
\hline Locus_3949_Transcript_61/161_Confidence_0.215_Length_7763 & FORWARD & 54.97 & \begin{tabular}{l|l|l}
45.45 & AGAAAGTAATCCCTCTCTCTCC \\
\end{tabular} & 137 & 425 & 425 & $\mathrm{AG}$ & AGAGAGAGAGAGAGAGAGAGAGAG & 24 \\
\hline Locus_3949_Transcript_61/161_Confidence_0.215_Length_7763 & REVERSE & 55.36 & 57.14 CGCTCTCTCTCTCTCTCTCTC & & & & & & \\
\hline Locus_3949_Transcript_61/161_Confidence_0.215_Length_7763 & FORWARD & 55.28 & $\begin{array}{lll}40 & \text { AGCACTTGATTCCAACAAAC } \\
\end{array}$ & 140 & 425 & 425 & $\mathrm{AG}$ & AGAGAGAGAGAGAGAGAGAGAGAG & 24 \\
\hline Locus_3949_Transcript_61/161_Confidence_0.215_Length_7763 & REVERSE & 54.67 & \begin{tabular}{l|l|l|}
33.33 & TGTCAACGATTTTCTCTTTTC \\
\end{tabular} & & & & & & \\
\hline Locus_3949_Transcript_61/161_Confidence_0.215_Length_7763 & FORWARD & 54.67 & \begin{tabular}{l|l|}
33.33 & GAAAAGAGAAAATCGTTGACA \\
\end{tabular} & 136 & 425 & 425 & $\mathrm{AG}$ & AGAGAGAGAGAGAGAGAGAGAG & 22 \\
\hline Locus_3949_Transcript_61/161_Confidence_0.215_Length_7763 & REVERSE & 54.65 & \begin{tabular}{l|l|} 
42.86 & ATCTTTCGGACTAAGTGGAGT \\
\end{tabular} & & & & & & \\
\hline Locus_3964_Transcript_42/46_Confidence_0.543_Length_1667 & FORWARD & 54.95 & \begin{tabular}{l|l|}
40 & CACGACAAATACAGCAGAAA \\
\end{tabular} & 230 & 420 & 420 & GA & GAGAGAGAGAGAGAGAGAGA & 20 \\
\hline Locus_3964_Transcript_42/46_Confidence_0.543_Length_1667 & REVERSE & 56.44 & \begin{tabular}{l|l|} 
42.86 & ATCATTCACCTCCACATTCTC \\
\end{tabular} & & & & & & \\
\hline Locus_3997_Transcript_26/50_Confidence_0.520_Length_3952 & FORWARD & 55.16 & \begin{tabular}{l|l|}
35 & TTTTGAGCTCGGGATATTTA \\
\end{tabular} & 219 & 421 & 421 & AT & ATATATATATATATATATAT & 20 \\
\hline Locus_3997_Transcript_26/50_Confidence_0.520_Length_3952 & REVERSE & 54.97 & \begin{tabular}{l|l|l}
33.33 & ATCACAAAAACCAATCACAAC \\
\end{tabular} & & & & & & \\
\hline Locus_4045_Transcript_3/25_Confidence_0.571_Length_1040 & FORWARD & 55.09 & \begin{tabular}{l|l|} 
42.86 & ATCATCATCTCCCTAACCTGT \\
\end{tabular} & 153 & 425 & 425 & $\mathrm{AG}$ & AGAGAGAGAGAGAGAGAGAGAGAG & 24 \\
\hline Locus_4045_Transcript_3/25_Confidence_0.571_Length_1040 & REVERSE & 54.67 & \begin{tabular}{l|l|l}
33.33 & TGTCAACGATTTTCTCTTTTC \\
\end{tabular} & & & & & & \\
\hline Locus_4045_Transcript_3/25_Confidence_0.571_Length_1040 & FORWARD & 54.94 & \begin{tabular}{l|l}
55 & GTCGGTCACAAGAGAGAGAG \\
\end{tabular} & 131 & 425 & 425 & $\mathrm{AG}$ & AGAGAGAGAGAGAGAGAGAGAG & 22 \\
\hline Locus_4045_Transcript_3/25_Confidence_0.571_Length_1040 & REVERSE & 54.84 & \begin{tabular}{l|l|}
38.1 & TCTCTAAGCACCATAAAATGC \\
\end{tabular} & & & & & & \\
\hline Locus_4149_Transcript_59/95_Confidence_0.482_Length_2275 & FORWARD & 55.21 & \begin{tabular}{l|l|}
55 & ATCTCAGAGCTCTCCCTCTC \\
\end{tabular} & 150 & 424 & 424 & $\mathrm{CT}$ & СТСТСТСТСТСТСТСТСТСТСТ & 22 \\
\hline Locus_4149_Transcript_59/95_Confidence_0.482_Length_2275 & REVERSE & 55.09 & \begin{tabular}{l|l|}
47.62 & GGTATAGGGGATTGGTATCAG \\
\end{tabular} & & & & & & \\
\hline Locus_4149_Transcript_59/95_Confidence_0.482_Length_2275 & FORWARD & 54.14 & \begin{tabular}{l|l}
38.1 & TCCTAAAAAGGTTACAAGCTG \\
\end{tabular} & 148 & 424 & 424 & $\mathrm{CT}$ & СТСТСТСТСТСТСТСТСТСТСТСТ & 24 \\
\hline Locus_4149_Transcript_59/95_Confidence_0.482_Length_2275 & REVERSE & 54.69 & \begin{tabular}{l|l}
47.62 & TGTAAGAGCGAGAGAGAGAGA \\
\end{tabular} & & & & & & \\
\hline Locus_4190_Transcript_40/57_Confidence_0.503_Length_2043 & FORWARD & 55.18 & \begin{tabular}{l|l}
38.1 & ATTGGATCCTAACCACAACTT \\
\end{tabular} & 131 & 423 & 423 & $\mathrm{AG}$ & AGAGAGAGAGAGAGAGAGAGAG & 22 \\
\hline Locus_4190_Transcript_40/57_Confidence_0.503_Length_2043 & REVERSE & 55.36 & \begin{tabular}{l|l|l|}
57.14 & CGCTCTCTCTCTCTCTCTCTC \\
\end{tabular} & & & & & & \\
\hline Locus_4236_Transcript_27/36_Confidence_0.528_Length_2128 & FORWARD & 54.24 & \begin{tabular}{l|l}
38.1 & GATGAAGTTTATGAAGCTTGG \\
\end{tabular} & 154 & 423 & 423 & TC & TCTCTCTСТСТСТСТСТСТСТC & 22 \\
\hline Locus_4236_Transcript_27/36_Confidence_0.528_Length_2128 & REVERSE & 54.82 & \begin{tabular}{l|l|} 
42.86 & TAGACCTGAACCATTGGAGTA \\
\end{tabular} & & & & & & \\
\hline Locus_4261_Transcript_34/58_Confidence_0.436_Length_1469 & FORWARD & 55.03 & \begin{tabular}{l|l|}
33.33 & CAGAATTTCATTGATTTCAGC \\
\end{tabular} & 144 & 423 & 423 & $\mathrm{TC}$ & TCTCTCTCТСТСТСТСТСТСТС & 22 \\
\hline Locus_4261_Transcript_34/58_Confidence_0.436_Length_1469 & REVERSE & 54.83 & \begin{tabular}{l|l}
38.1 & CACTTCCCTTAATCCCATATT \\
\end{tabular} & & & & & & \\
\hline Locus_4262_Transcript_18/28_Confidence_0.488_Length_918 & FORWARD & 55.76 & \begin{tabular}{l|l|}
52.38 & CGGAAGATCTCTCTCTCTCAC \\
\end{tabular} & 141 & 424 & 424 & $\mathrm{CT}$ & СТСТСТСТСТСТСТСТСТСТСТСТ & 24 \\
\hline Locus_4262_Transcript_18/28_Confidence_0.488_Length_918 & REVERSE & 55.08 & \begin{tabular}{l|l|}
47.62 & CTAGTAGGGGCAAGATGGTAT \\
\end{tabular} & & & & & & \\
\hline Locus_4313_Transcript_39/40_Confidence_0.515_Length_3949 & FORWARD & 55.23 & \begin{tabular}{l|l|}
38.1 & GTTTCTCCCTATTGTTTGCTT \\
\end{tabular} & 165 & 424 & 424 & $\mathrm{CT}$ & СТСТСТСТСТСТСТСТСТСТСТСТ & 24 \\
\hline Locus_4313_Transcript_39/40_Confidence_0.515_Length_3949 & REVERSE & 55.2 & \begin{tabular}{l|l|} 
42.86 & ATAGGGGATTGGTATCAGAGA \\
\end{tabular} & & & & & & \\
\hline Locus_4313_Transcript_39/40_Confidence_0.515_Length_3949 & FORWARD & 55.23 & \begin{tabular}{l|l|}
38.1 & GTTTCTCCCTATTGTTTGCTT \\
\end{tabular} & 172 & 424 & 424 & CT & СТСТСТСТСТСТСТСТСТСТСТСТ & 24 \\
\hline Locus_4313_Transcript_39/40_Confidence_0.515_Length_3949 & REVERSE & 55.09 & \begin{tabular}{l|l|}
42.86 & AGATGGTATAGGGGATTGGTA \\
\end{tabular} & & & & & & \\
\hline Locus_4316_Transcript_4/10_Confidence_0.661_Length_1547 & FORWARD & 55.27 & 38.1 CTGCAGCTGAAGAAAGTTAAA & 142 & 419 & 419 & $\mathrm{TG}$ & TGTGTGTGTGTGTGTGTG & 18 \\
\hline Locus_4316_Transcript_4/10_Confidence_0.661_Length_1547 & REVERSE & 55.12 & \begin{tabular}{l|l}
47.62 & GGTTATCCCACATACACACAC \\
\end{tabular} & & & & & & \\
\hline Locus_4316_Transcript_4/10_Confidence_0.661_Length_1547 & FORWARD & 55.27 & \begin{tabular}{l|l}
38.1 & CTGCAGCTGAAGAAAGTTAAA \\
\end{tabular} & 149 & 419 & 419 & $\mathrm{TG}$ & TGTGTGTGTGTGTGTGTG & 18 \\
\hline Locus_4316_Transcript_4/10_Confidence_0.661_Length_1547 & REVERSE & 53.84 & \begin{tabular}{l|l|}
33.33 & AAAAACAGGTTATCCCACATA \\
\end{tabular} & & & & & & \\
\hline Locus_4326_Transcript_18/42_Confidence_0.439_Length_1827 & FORWARD & 54.79 & \begin{tabular}{l|l|}
31.82 & CAAGCTTGTATTTCTTTTCCTT \\
\end{tabular} & 163 & 421 & 421 & $\mathrm{TC}$ & TCTCTCTCTCTCTCTCTCTC & 20 \\
\hline Locus_4326_Transcript_18/42_Confidence_0.439_Length_1827 & REVERSE & 55.14 & \begin{tabular}{l|l|}
33.33 & GTAATTCGGCCAATTCTTATT \\
\end{tabular} & & & & & & \\
\hline Locus_4354_Transcript_27/35_Confidence_0.646_Length_1571 & FORWARD & 54.89 & \begin{tabular}{l|l|l}
33.33 & TTAGATGGAACCAGAAAATCA \\
\end{tabular} & 135 & 425 & 425 & AT & ATATATATATATATATATATATAT & 24 \\
\hline
\end{tabular}




\begin{tabular}{|c|c|c|c|c|c|c|c|c|c|c|}
\hline Locus_4354_Transcript_27/35_Confidence_0.646_Length_1571 & REVERSE & 55.25 & 42.86 & TGTTCAACAGAGGATTGAGTC & & & & & & \\
\hline Locus_4431_Transcript_6/20_Confidence_0.656_Length_1078 & FORWARD & 55.28 & 38.1 & AAACCCCCTCAATACTCATAA & 139 & 427 & 427 & GA & GAGAGAGAGAGAGAGAGAGAGAGAGA & 26 \\
\hline Locus_4431_Transcript_6/20_Confidence_0.656_Length_1078 & REVERSE & 55.36 & 57.14 & CGCTCTCTCTCTCTCTCTCTC & & & & & & \\
\hline Locus_4431_Transcript_6/20_Confidence_0.656_Length_1078 & FORWARD & 55.44 & 52.17 & GAGAGAGAGAGAGAGAGGAGAGA & 156 & 427 & 427 & $\mathrm{AG}$ & AGAGAGAGAGAGAGAGAGAGAGAG & 24 \\
\hline Locus_4431_Transcript_6/20_Confidence_0.656_Length_1078 & REVERSE & 56.25 & 38.1 & GCTGTCAACGATTTTCTCTTT & & & & & & \\
\hline Locus_4431_Transcript_6/20_Confidence_0.656_Length_1078 & FORWARD & 55.28 & 38.1 & ACССССТCAATACTCATAAAA & 137 & 427 & 427 & GA & GAGAGAGAGAGAGAGAGAGAGAGA & 24 \\
\hline Locus_4431_Transcript_6/20_Confidence_0.656_Length_1078 & REVERSE & 54.28 & 47.62 & GCTTTTTCTCCTCTCTCTCTC & & & & & & \\
\hline Locus_4431_Transcript_6/20_Confidence_0.656_Length_1078 & FORWARD & 55.28 & 42.86 & CССCСТCAATACTCATAAAAC & 162 & 427 & 427 & $\mathrm{AG}$ & AGAGAGAGAGAGAGAGAGAGAG & 22 \\
\hline Locus_4431_Transcript_6/20_Confidence_0.656_Length_1078 & REVERSE & 55.86 & 33.33 & GGGGTTTTTGAAGAAACATTA & & & & & & \\
\hline Locus_4431_Transcript_6/20_Confidence_0.656_Length_1078 & FORWARD & 54.42 & 43.48 & CAAATTGAGAGAGAGAGAGAGAG & 195 & 422 & 422 & GA & GAGAGAGAGAGAGAGAGAGAGA & 22 \\
\hline Locus_4431_Transcript_6/20_Confidence_0.656_Length_1078 & REVERSE & 54.65 & 40 & GCTGTCAACGATTTTCTCTT & & & & & & \\
\hline Locus_4464_Transcript_5/19_Confidence_0.670_Length_1168 & FORWARD & 53.96 & 38.1 & AAAGATGGTATAGGGGATTTC & 153 & 422 & 422 & $\mathrm{AG}$ & AGAGAGAGAGAGAGAGAG & 18 \\
\hline Locus_4464_Transcript_5/19_Confidence_0.670_Length_1168 & REVERSE & 54.53 & 42.86 & TCAATCTCTCAATCTCTCCAG & & & & & & \\
\hline Locus_4464_Transcript_5/19_Confidence_0.670_Length_1168 & FORWARD & 54.94 & 55 & GTCGGTCACAAGAGAGAGAG & 140 & 422 & 422 & $\mathrm{AG}$ & AGAGAGAGAGAGAGAGAGAGAG & 22 \\
\hline Locus_4464_Transcript_5/19_Confidence_0.670_Length_1168 & REVERSE & 55.03 & 38.1 & TCGACCTTATCAATCTCTCAA & & & & & & \\
\hline Locus_4473_Transcript_17/24_Confidence_0.561_Length_4523 & FORWARD & 55.42 & 42.86 & GAAGAATCTCCCTTTGAACAC & 171 & 422 & 422 & $\mathrm{CT}$ & СТСТСТСТСТСТСТСТСТСТСТ & 22 \\
\hline Locus_4473_Transcript_17/24_Confidence_0.561_Length_4523 & REVERSE & 55.2 & 42.86 & ATAGGGGATTGGTATCAGAGA & & & & & & \\
\hline Locus_4473_Transcript_17/24_Confidence_0.561_Length_4523 & FORWARD & 55.58 & 52.38 & TATCGCCTCTCTCTCTCTCTC & 151 & 422 & 422 & $\mathrm{CT}$ & СТСТСТСТСТСТСТСТСТСТСТ & 22 \\
\hline Locus_4473_Transcript_17/24_Confidence_0.561_Length_4523 & REVERSE & 55.09 & 47.62 & GGTATAGGGGATTGGTATCAG & & & & & & \\
\hline Locus_4473_Transcript_17/24_Confidence_0.561_Length_4523 & FORWARD & 55.42 & 42.86 & GAAGAATCTCCCTTTGAACAC & 171 & 423 & 423 & $\mathrm{CT}$ & TCTCTCTCTCT & 22 \\
\hline Locus_4473_Transcript_17/24_Confidence_0.561_Length_4523 & REVERSE & 55.09 & 42.86 & AGATGGTATAGGGGATTGGTA & & & & & & \\
\hline Locus_4503_Transcript_3/8_Confidence_0.706_Length_3834 & FORWARD & 54.46 & 38.1 & CCACCAAACATTACCTAGAAA & 161 & 418 & 418 & $\mathrm{AT}$ & ATAT & 18 \\
\hline Locus_4503_Transcript_3/8_Confidence_0.706_Length_3834 & REVERSE & 55.35 & 33.33 & ACCAAGGTTTACGATGAATTT & & & & & & \\
\hline Locus_4590_Transcript_15/20_Confidence_0.677_Length_749 & FORWARD & 55.09 & 47.62 & GGTATAGGGGATTGGTATCAG & 182 & 419 & 419 & $\mathrm{AG}$ & AGAGAGAGAGAGAGAGAG & 18 \\
\hline Locus_4590_Transcript_15/20_Confidence_0.677_Length_749 & REVERSE & 54.02 & 42.86 & TGAGTTATATGCGTGAGTGAG & & & & & & \\
\hline Locus_4605_Transcript_13/17_Confidence_0.628_Length_1567 & FORWARD & 54.62 & 42.86 & TTTGACTGAAGATGGAGAGAG & 159 & 419 & 419 & $\mathrm{CT}$ & СТCTCTCTCTCTCTCTCT & 18 \\
\hline Locus_4605_Transcript_13/17_Confidence_0.628_Length_1567 & REVERSE & 55.23 & 38.1 & AAAGAACAAGTTATGCCTTCC & & & & & & \\
\hline Locus_4628_Transcript_23/40_Confidence_0.525_Length_923 & FORWARD & 54.79 & 33.33 & AGTGAAGCATGACATGAAAAT & 155 & 424 & 424 & $\mathrm{AG}$ & AGAGAGAGAGAGAGAGAGAGAGAG & 24 \\
\hline Locus_4628_Transcript_23/40_Confidence_0.525_Length_923 & REVERSE & 55.28 & 52.38 & GTCTTCCACTCTCTACCTGCT & & & & & & \\
\hline Locus_4643_Transcript_16/20_Confidence_0.626_Length_1411 & FORWARD & 55.31 & 38.1 & CGTAAAACACTGCAGAAAAAC & 161 & 424 & 424 & $\mathrm{AT}$ & ATATATATATATATATATATATAT & 24 \\
\hline Locus_4643_Transcript_16/20_Confidence_0.626_Length_1411 & REVERSE & 54.79 & 38.1 & CTGATTCAATCCAGCTCTAAA & & & & & & \\
\hline Locus_4645_Transcript_37/45_Confidence_0.537_Length_6396 & FORWARD & 55 & 42.86 & AAGGTGACCATATTCTGTGTG & 145 & 421 & 421 & $\mathrm{TC}$ & TCTCTCTCTCTCTCTCTCTC & 20 \\
\hline Locus_4645_Transcript_37/45_Confidence_0.537_Length_6396 & REVERSE & 55.28 & 38.1 & ATTCAAATTAGCTCGGGATAG & & & & & & \\
\hline Locus_4738_Transcript_98/111_Confidence_0.419_Length_2232 & FORWARD & 55.06 & 42.86 & AAATCTACCCTGTTCGTTCTC & 156 & 425 & 425 & GA & GAGAGAGAGAGAGAGAGAGAGAGA & 24 \\
\hline Locus_4738_Transcript_98/111_Confidence_0.419_Length_2232 & REVERSE & 55.36 & 57.14 & CGCTCTCTCTCTCTCTCTCTC & & & & & & \\
\hline Locus_4738_Transcript_98/111_Confidence_0.419_Length_2232 & FORWARD & 54.37 & 52.38 & GAGATAGCGAGTGAGTGAGAG & 150 & 425 & 425 & $\mathrm{AG}$ & AGAGAGAGAGAGAGAGAGAGAGAG & 24 \\
\hline Locus_4738_Transcript_98/111_Confidence_0.419_Length_2232 & REVERSE & 54.67 & 33.33 & TGTCAACGATTTTCTCTTTTC & & & & & & \\
\hline Locus_4738_Transcript_98/111_Confidence_0.419_Length_2232 & FORWARD & 54.67 & 33.33 & GAAAAGAGAAAATCGTTGACA & 148 & 425 & 425 & $\mathrm{AG}$ & AGAGAGAGAGAGAGAGAGAGAG & 22 \\
\hline Locus_4738_Transcript_98/111_Confidence_0.419_Length_2232 & REVERSE & 55.26 & 45 & GAACACAGCATGGTTCTTCT & & & & & & \\
\hline Locus_4738_Transcript_98/111_Confidence_0.419_Length_2232 & FORWARD & 54.37 & 52.38 & GTGAGAGAGAGATAGCGAGTG & 158 & 422 & 422 & GA & GAGAGAGAGAGAGAGAGAGAGAGA & 24 \\
\hline Locus_4738_Transcript_98/111_Confidence_0.419_Length_2232 & REVERSE & 54.67 & 33.33 & TGTCAACGATTTTCTCTTTTC & & & & & & \\
\hline Locus_4775_Transcript_12/14_Confidence_0.411_Length_569 & FORWARD & 53.86 & 23.81 & TTTTCTTGCTTGAAAGTTTTT & 159 & 424 & 424 & $\mathrm{TA}$ & TATATATATATATATATATATATA & 24 \\
\hline Locus_4775_Transcript_12/14_Confidence_0.411_Length_569 & REVERSE & 56.55 & 45 & AGTCCTCCCCCAGATAATTT & & & & & & \\
\hline Locus_4775_Transcript_12/14_Confidence_0.411_Length_569 & FORWARD & 54.1 & 26.09 & TCTTTTACTTTGAACAATGTTTG & 156 & 424 & 424 & $\mathrm{TA}$ & TATATATATATATATATATATATATA & 26 \\
\hline Locus_4775_Transcript_12/14_Confidence_0.411_Length_569 & REVERSE & 53.84 & 42.86 & ATACACATATGACCCCTGTTC & & & & & & \\
\hline
\end{tabular}


Locus_4775_Transcript_12/14_Confidence_0.411_Length_569 Locus_4775_Transcript_12/14_Confidence_0.411_Length_569 Locus_4799_Transcript_10/13_Confidence_0.656_Length_1905 Locus_4799_Transcript_10/13_Confidence_0.656_Length_1905 Locus_4803_Transcript_22/29_Confidence_0.592_Length_2489 Locus_4803_Transcript_22/29_Confidence_0.592_Length_2489 Locus_4803_Transcript_22/29_Confidence_0.592_Length_2489 Locus_4803_Transcript_22/29_Confidence_0.592_Length_2489 Locus_4803_Transcript_22/29_Confidence_0.592_Length_2489 Locus_4803_Transcript_22/29_Confidence_0.592_Length_2489 Locus_4829_Transcript_2/9 Confidence 0.692 Length_2752 Locus_4829_Transcript_2/9_Confidence_0.692_Length_2752 Locus_4872_Transcript_10/26_Confidence_0.577_Length_1460 Locus_4872_Transcript_10/26_Confidence_0.577_Length_1460 Locus_4903_Transcript_12/82_Confidence_0.411_Length_1608 Locus_4903_Transcript_12/82_Confidence_0.411_Length_1608 Locus_5030_Transcript_17/56_Confidence_0.535_Length_1214 Locus_5030_Transcript_17/56_Confidence_0.535_Length_1214 Locus 5030 Transcript $17 / 56$ Confidence 0.535 Length 1214 Locus_5030_Transcript_17/56_Confidence_0.535_Length_1214 Locus_5086_Transcript_68/93_Confidence_0.310_Length_2248 Locus_5086_Transcript_68/93_Confidence_0.310_Length_2248 Locus_5108_Transcript_7/49_Confidence_0.566_Length_1833 Locus_5108_Transcript_7/49_Confidence_0.566_Length_1833 Locus_5108_Transcript_7/49_Confidence_0.566_Length_1833 Locus_5108_Transcript_7/49_Confidence_0.566_Length_1833 Locus_5122 Transcript 4/27_Confidence 0.659 Length_2886 Locus_5122_Transcript_4/27_Confidence_0.659_Length_2886 Locus_5264_Transcript_8/13_Confidence_0.664_Length_2616 Locus_5264_Transcript_8/13_Confidence_0.664_Length_2616 Locus 5334 Transcript 22/44 Confidence 0.604 Length 2169 Locus_5334_Transcript_22/44_Confidence_0.604_Length_2169 Locus_5353_Transcript_9/10_Confidence_0.725_Length_2275 Locus_5353_Transcript 9/10_Confidence_0.725_Length_2275 Locus_5372_Transcript_22/29_Confidence_0.505_Length_3049 Locus_5372_Transcript_22/29_Confidence_0.505_Length_3049 Locus_5426_Transcript_22/26_Confidence_0.571_Length_799 Locus_5426_Transcript_22/26_Confidence_0.571_Length_799 Locus_5493_Transcript_69/82_Confidence_0.534_Length_5620 Locus_5493_Transcript_69/82_Confidence_0.534_Length_5620 Locus_5493_Transcript_69/82_Confidence_0.534_Length_5620 Locus 5493 Transcript 69/82 Confidence 0.534_Length 5620 Locus_5493_Transcript_69/82_Confidence_0.534_Length_5620 Locus_5493_Transcript_69/82_Confidence_0.534_Length_5620 Locus_5499_Transcript_18/24_Confidence_0.563_Length_2811

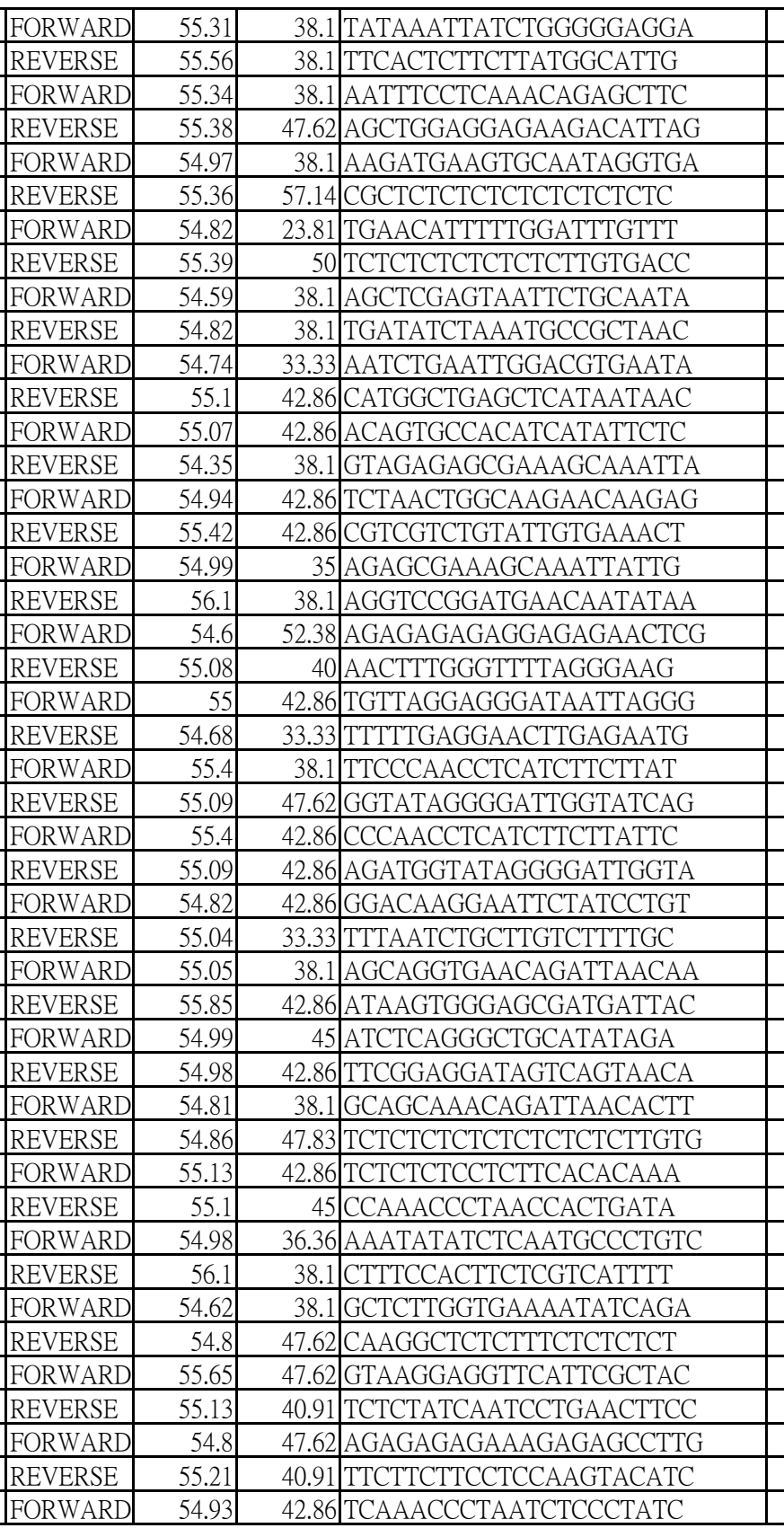

\begin{tabular}{l} 
I \\
I \\
\hline \\
\hline \\
\hline \\
\hline
\end{tabular}

\begin{tabular}{|c|c|c|c|c|c|}
\hline 156 & 424 & 424 & TA & TATATATATATATATATA & 18 \\
\hline 158 & 418 & 418 & $\mathrm{TC}$ & TCTCТСТСТСТСТСТСТС & 18 \\
\hline & & & & & \\
\hline 151 & 422 & 422 & $\mathrm{AG}$ & AGAGAGAGAGAGAGAGAGAGAG & 22 \\
\hline 151 & 422 & 422 & $\mathrm{AG}$ & AGAGAGAGAGAGAGAGAGAGAGAG & 24 \\
\hline & & & & & \\
\hline 189 & 422 & 422 & $\mathrm{AG}$ & AGAGAGAGAGAGAGAGAG & 18 \\
\hline 144 & 421 & 421 & AT & \begin{tabular}{|l} 
ATATATATATATATATATAT \\
\end{tabular} & 20 \\
\hline & & & & & \\
\hline 148 & 426 & 426 & CT & СТСТСТСТСТСТСТСТСТСТСТСТСТ & 26 \\
\hline 144 | & 423 & 423 & $\mathrm{CT}$ & СТСТСТСТСТСТСТСТСТСТСТ & 22 \\
\hline & & & & & \\
\hline 135 & 423 & 423 & GA & GAGAGAGAGAGAGAGAGAGAGA & 22 \\
\hline & & & & & \\
\hline 138 & 423 & 423 & $\mathrm{AG}$ & AGAGAGAGAGAGAGAGAG & 18 \\
\hline 154 & 425 & 425 & $\mathrm{AG}$ & AGAGAGAGAGAGAGAGAGAGAGAG & 24 \\
\hline 163 & 424 & 424 & TC & ТСТСТСТСТСТСТСТСТСТС & 20 \\
\hline & & & & & \\
\hline 165 & 424 & 424 & CT & СТСТСТСТСТСТСТСТСТСТСТСТ & 24 \\
\hline 147 & 410 & 419 & $A C$ & ACACACACACACACACAC & 18 \\
\hline & & & & & \\
\hline 147 & 420 & 420 & AT & ATATATATATATATATATAT & 20 \\
\hline 150 & 421 & 421 & GT & \begin{tabular}{|l} 
GTGTGTGTGTGTGTGTGTGT \\
\end{tabular} & 20 \\
\hline & & & & & \\
\hline 122 & 419 & 419 & GA & GAGAGAGAGAGAGAGAGAGAGA & 22 \\
\hline 154 & 418 & 418 & CT & СТСТСТСТСТСТСТСТСТ & 18 \\
\hline & & & & & \\
\hline 131 & 423 & 423 & GA & GAGAGAGAGAGAGAGAGAGAGA & 22 \\
\hline & & & & & \\
\hline 150 & 419 & 419| & $\mathrm{AG}$ & AGAGAGAGAGAGAGAGAG & 18 \\
\hline 152 & 419 & 419 & $\Delta G_{\mathrm{t}}$ & AGAGAGAGAGAGAGAGAG & 18 \\
\hline & & & & & \\
\hline 138 & 419 & 419 & $\mathrm{AG}$ & AGAGAGAGAGAGAGAGAG & 18 \\
\hline & & & & & \\
\hline 152 & 419 & 419 & CT & СТСТСТСТСТСТСТСТСТ & 18 \\
\hline
\end{tabular}




\begin{tabular}{|c|c|c|c|c|c|c|c|c|c|c|}
\hline Locus_5499_Transcript_18/24_Confidence_0.563_Length_2811 & REVERSE & 55.09 & 35 & AGAAAAATCAGTTTGCTGGA & & & & & & \\
\hline Locus_5504_Transcript_5/23_Confidence_0.597_Length_2864 & FORWARD & 54.87 & 33.33 & GGAAAACCAAAGATTACCATT & 143 & 420 & 420 & AT & ATATATATATATATATATAT & 20 \\
\hline Locus_5504_Transcript_5/23_Confidence_0.597_Length_2864 & REVERSE & 54.02 & 38.1 & TCTTGTAGTGACCAATCATCA & & & & & & \\
\hline Locus_5524_Transcript_28/34_Confidence_0.559_Length_1654 & FORWARD & 53.96 & 28.57 & GAAAGCAAATTATTGAAGGAA & 133 & 423 & 423 & GA & GAGAGAGAGAGAGAGAGAGAGA & 22 \\
\hline Locus_5524_Transcript_28/34_Confidence_0.559_Length_1654 & REVERSE & 54.67 & 33.33 & TGTCAACGATTTTCTCTTTTC & & & & & & \\
\hline Locus_5524_Transcript_28/34_Confidence_0.559_Length_1654 & FORWARD & 53.96 & 28.57 & GAAAGCAAATTATTGAAGGAA & 178 & 423 & 423 & $\mathrm{AG}$ & AGAGAGAGAGAGAGAGAG & 18 \\
\hline Locus_5524_Transcript_28/34_Confidence_0.559_Length_1654 & REVERSE & 55.63 & 52.38 & CGGATCTACAACTCTCCTCTC & & & & & & \\
\hline Locus_5524_Transcript_28/34_Confidence_0.559_Length_1654 & FORWARD & 54.67 & 33.33 & GAAAAGAGAAAATCGTTGACA & 137 & 423 & 423 & $\mathrm{AG}$ & AGAGAGAGAGAGAGAGAGAGAG & 22 \\
\hline Locus_5524_Transcript_28/34_Confidence_0.559_Length_1654 & REVERSE & 55.44 & 42.86 & CTGAGTCAATGGTGAGAGAAA & & & & & & \\
\hline Locus_5599_Transcript_8/16_Confidence_0.544_Length_537 & FORWARD & 56.3 & 42.86 & GCCAAATTCTGATCCAGTAAC & 150 & 418 & 418 & GA & GAGAGAGAGAGAGAGAGA & 18 \\
\hline Locus_5599_Transcript_8/16_Confidence_0.544_Length_537 & REVERSE & 55.45 & 42.86 & AGATTCAGTACCATCCGCTAT & & & & & & \\
\hline Locus_5657_Transcript_52/60_Confidence_0.469_Length_1878 & FORWARD & 54.24 & 42.86 & AATGAAAGTCTTCTCCCTCTC & 151 & 422 & 422 & CT & СТСТСТСТСТСТСТСТСТСТСТ & 22 \\
\hline Locus_5657_Transcript_52/60_Confidence_0.469_Length_1878 & REVERSE & 55.09 & 42.86 & AGATGGTATAGGGGATTGGTA & & & & & & \\
\hline Locus_5666_Transcript_13/30_Confidence_0.506_Length_2588 & FORWARD & 54.8 & 38.1 & TTCTTTGATCTACACGTCCAT & 160 & 421 & 421 & $\mathrm{TC}$ & TСТСТСТСТСТСТСТСТСТС & 20 \\
\hline Locus_5666_Transcript_13/30_Confidence_0.506_Length_2588 & REVERSE & 55.5 & 47.62 & GAGAGAGGAGCTGGTTTTCTA & & & & & & \\
\hline Locus_5780_Transcript_23/33_Confidence_0.563_Length_1962 & FORWARD & 54.52 & 33.33 & CTGCATAAATTGTCCTGATTT & 136 & 419 & 419 & $\mathrm{AC}$ & ACACACACACACACACAC & 18 \\
\hline Locus_5780_Transcript_23/33_Confidence_0.563_Length_1962 & REVERSE & 54.76 & 28.57 & GCTTCATTTGGTTTTGTTTTA & & & & & & \\
\hline Locus_5867_Transcript_12/24_Confidence_0.429_Length_1461 & FORWARD & 55.39 & 38.1 & ATAAGCAAAAGGGAGTTCAAG & 150 & 423 & 423 & GA & GAGAGAGAGAGAGAGAGAGAGA & 22 \\
\hline Locus_5867_Transcript_12/24_Confidence_0.429_Length_1461 & REVERSE & 55.36 & 57.14 & CGCTCTCTCTCTCTCTCTCTC & & & & & & \\
\hline Locus_5867_Transcript_12/24_Confidence_0.429_Length_1461 & FORWARD & 55.49 & 42.86 & AGCGAGAAATCTAAGCCATAC & 175 & 423 & 423 & $\mathrm{AG}$ & AGAGAGAGAGAGAGAGAGAGAGAG & 24 \\
\hline Locus_5867_Transcript_12/24_Confidence_0.429_Length_1461 & REVERSE & 55.22 & 47.62 & TGGGATCTCTCTTTCTCTCTC & & & & & & \\
\hline Locus_5867_Transcript_12/24_Confidence_0.429_Length_1461 & FORWARD & 54.25 & 38.1 & GAAAGAGAAAATCGTTGACAG & 153 & 423 & 423 & $\mathrm{AG}$ & AGAGAGAGAGAGAGAGAGAGAG & 22 \\
\hline Locus_5867_Transcript_12/24_Confidence_0.429_Length_1461 & REVERSE & 55.34 & 47.62 & TGACTCAGCAGTCCTCTCTTA & & & & & & \\
\hline Locus_5931_Transcript_12/22_Confidence_0.654_Length_1580 & FORWARD & 54.99 & 40 & TGTTTCGTCCTCAGAAGTTT & 148 & 419 & 419 & $\mathrm{TC}$ & TCTCTCTCTCTCTCTCTC & 18 \\
\hline Locus_5931_Transcript_12/22_Confidence_0.654_Length_1580 & REVERSE & 54.89 & 36.36 & CAAGTAAACGAAGAAGACAACA & & & & & & \\
\hline Locus_6033_Transcript_8/11_Confidence_0.660_Length_977 & FORWARD & 54.55 & 38.1 & TAATTAAGCACACGTCTCACA & 149 & 419 & 419 & $\mathrm{TA}$ & TATATATATATATATATA & 18 \\
\hline Locus_6033_Transcript_8/11_Confidence_0.660_Length_977 & REVERSE & 54.05 & 33.33 & TCCCGATTAGGAACATATAAA & & & & & & \\
\hline Locus_6093_Transcript_12/19_Confidence_0.648_Length_1495 & FORWARD & 55.91 & 42.86 & CCGAATAGGAAGATTCTGTGT & 161 & 418 & 418 & $\mathrm{AG}$ & AGAGAGAGAGAGAGAGAG & 18 \\
\hline Locus_6093_Transcript_12/19_Confidence_0.648_Length_1495 & REVERSE & 56.3 & 50 & GCTAGAGATCGGATCAGCTT & & & & & & \\
\hline Locus_6256_Transcript_39/47_Confidence_0.548_Length_1587 & FORWARD & 54.84 & 57.14 & GAGAGTGGGAGAGAGAGAGAG & 146 & 424 & 424 & GA & GAGAGAGAGAGAGAGAGAGAGAGA & 24 \\
\hline Locus_6256_Transcript_39/47_Confidence_0.548_Length_1587 & REVERSE & 55.3 & 28.57 & TTTTTCCTTCAGCACAAATTA & & & & & & \\
\hline Locus_6274_Transcript_13/17_Confidence_0.642_Length_1149 & FORWARD & 55.06 & 38.1 & TCCAAATCCTGTGTTGTATTC & 142 & 422 & 422 & $\mathrm{AG}$ & AGAGAGAGAGAGAGAGAGAGAG & 22 \\
\hline Locus_6274_Transcript_13/17_Confidence_0.642_Length_1149 & REVERSE & 54.43 & 38.1 & CACACATTCCAAACTTCTTCT & & & & & & \\
\hline Locus_6308_Transcript_28/31_Confidence_0.502_Length_1512 & FORWARD & 56.94 & 50 & CACGAAGACTCCAGAAGGTT & 148 & 421 & 421 & $\mathrm{TC}$ & TCTCTCTCTCTCTCTCTCTC & 20 \\
\hline Locus_6308_Transcript_28/31_Confidence_0.502_Length_1512 & REVERSE & 54.83 & 38.1 & AAAAGATGGTATAGGGGATTG & & & & & & \\
\hline Locus_6308_Transcript_28/31_Confidence_0.502_Length_1512 & FORWARD & 55.39 & 47.62 & CCTGTAAATCCCATCTCTCTC & 148 & 421 & 421 & $\mathrm{CT}$ & СТСТСТСТСТСТСТСТСТСТСТСТ & 24 \\
\hline Locus_6308_Transcript_28/31_Confidence_0.502_Length_1512 & REVERSE & 55.23 & 42.86 & CATCTACAACAAGAAGGCAAG & & & & & & \\
\hline Locus_6531_Transcript_1/15_Confidence_0.540_Length_913 & FORWARD & 54.46 & 38.1 & GGAAACCAACAGTAAACAAGA & 132 & 425 & 425 & $\mathrm{AG}$ & AGAGAGAGAGAGAGAGAGAGAGAG & 24 \\
\hline Locus_6531_Transcript_1/15_Confidence_0.540_Length_913 & REVERSE & 54.67 & 33.33 & TGTCAACGATTTTCTCTTTTC & & & & & & \\
\hline Locus_6531_Transcript_1/15_Confidence_0.540_Length_913 & FORWARD & 54.49 & 50 & GGATCAGAGAGAGAGAGAGAGA & 159 & 425 & 425 & $\mathrm{AG}$ & AGAGAGAGAGAGAGAGAGAGAG & 22 \\
\hline Locus_6531_Transcript_1/15_Confidence_0.540_Length_913 & REVERSE & 55.31 & 38.1 & GCCAAATCTACGCCTATTTAT & & & & & & \\
\hline Locus_6531_Transcript_1/15_Confidence_0.540_Length_913 & FORWARD & 54.46 & 38.1 & GGAAACCAACAGTAAACAAGA & 132 & 424 & 424 & $\mathrm{AG}$ & AGAGAGAGAGAGAGAGAGAGAGAG & 24 \\
\hline Locus_6531_Transcript_1/15_Confidence_0.540_Length_913 & REVERSE & 54.67 & 33.33 & TGTCAACGATTTTCTCTTTTC & & & & & & \\
\hline Locus_6531_Transcript_1/15_Confidence_0.540_Length_913 & FORWARD & 54.46 & 38.1 & GGAAACCAACAGTAAACAAGA & 175 & 424 & 424 & $\mathrm{AG}$ & AGAGAGAGAGAGAGAGAG & 18 \\
\hline Locus_6531_Transcript_1/15_Confidence_0.540_Length_913 & REVERSE & 55 & 47.62 & CGCCTATTTATCTCCTCTCTC & & & & & & \\
\hline
\end{tabular}


Locus_6531_Transcript_1/15_Confidence_0.540_Length_913 Locus_6531_Transcript_1/15_Confidence_0.540_Length 913

Locus_6565_Transcript_3/20_Confidence_0.630_Length_1278

Locus_6565_Transcript_3/20_Confidence_0.630_Length_1278 Locus_6565_Transcript_3/20_Confidence_0.630_Length_1278 Locus 6565 Transcript 3/20 Confidence 0.630 Length 1278 Locus_6614_Transcript 5/6_Confidence_0.886_Length_2115 Locus_6614_Transcript_5/6_Confidence_0.886_Length_2115 Locus_6632_Transcript_34/37_Confidence_0.605_Length_2723 Locus_6632_Transcript_34/37_Confidence_0.605_Length_2723 Locus 6632 Transcript 34/37 Confidence 0.605 Length 2723 Locus_6632_Transcript_34/37_Confidence_0.605_Length_2723 Locus_6632_Transcript_34/37_Confidence_0.605_Length_2723 Locus_6632_Transcript_34/37_Confidence_0.605_Length_2723 Locus_6632_Transcript_34/37_Confidence_0.605_Length_2723 Locus_6632_Transcript_34/37_Confidence_0.605_Length_2723 Locus_6651_Transcript_10/22_Confidence_0.628_Length_4410 Locus_6651_Transcript_10/22_Confidence_0.628_Length_4410 Locus_6680_Transcript_3/3_Confidence_0.600_Length_340 Locus_6680_Transcript_3/3_Confidence_0.600_Length_340 Locus_6978_Transcript_10/30_Confidence_0.526_Length_757 Locus_6978_Transcript_10/30_Confidence_0.526_Length_757 Locus_7044_Transcript_24/25_Confidence_0.599_Length_1748 Locus_7044_Transcript_24/25_Confidence_0.599_Length_1748 Locus_7044_Transcript_24/25_Confidence_0.599_Length_1748 Locus_7044_Transcript_24/25_Confidence_0.599_Length_1748 Locus 7232 Transcript $6 / 7$ Confidence 0.556 Length 844 Locus_7232_Transcript_6/7_Confidence_0.556_Length_844 Locus_7321_Transcript_22/28_Confidence_0.609_Length_1856 Locus_7321_Transcript_22/28_Confidence_0.609_Length_1856 Locus 7321 Transcript 22/28 Confidence 0.609 Length 1856 Locus_7321_Transcript_22/28_Confidence_0.609_Length_1856 Locus_7441_Transcript_11/33_Confidence_0.602_Length_1929 Locus_7441_Transcript_11/33_Confidence_0.602_Length_1929 Locus_7441_Transcript_11/33_Confidence_0.602_Length_1929 Locus_7441_Transcript_11/33_Confidence_0.602_Length_1929 Locus_7441_Transcript_11/33_Confidence_0.602_Length_1929 Locus_7441_Transcript_11/33_Confidence_0.602_Length_1929 Locus_7441_Transcript_11/33_Confidence_0.602_Length_1929 Locus_7441_Transcript_11/33_Confidence_0.602_Length_1929 Locus_7518_Transcript_13/14_Confidence_0.680_Length_7512 Locus_7518_Transcript_13/14_Confidence 0.680 Length 7512 Locus 7560 Transcript 20/20 Confidence 0.400 Length_1860 Locus_7560_Transcript_20/20_Confidence_0.400_Length_1869 Locus_7560_Transcript_20/20_Confidence_0.400_Length_1869

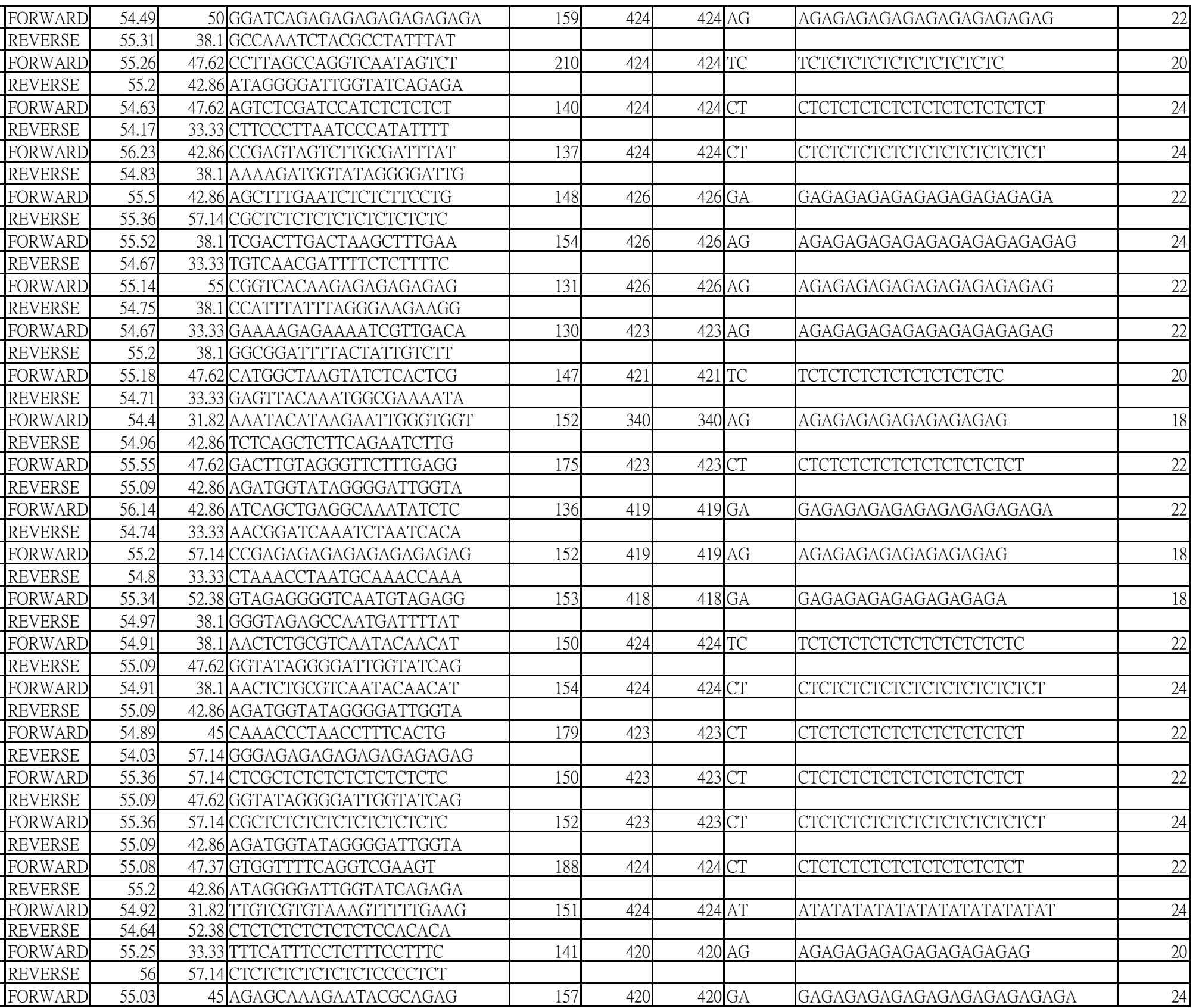




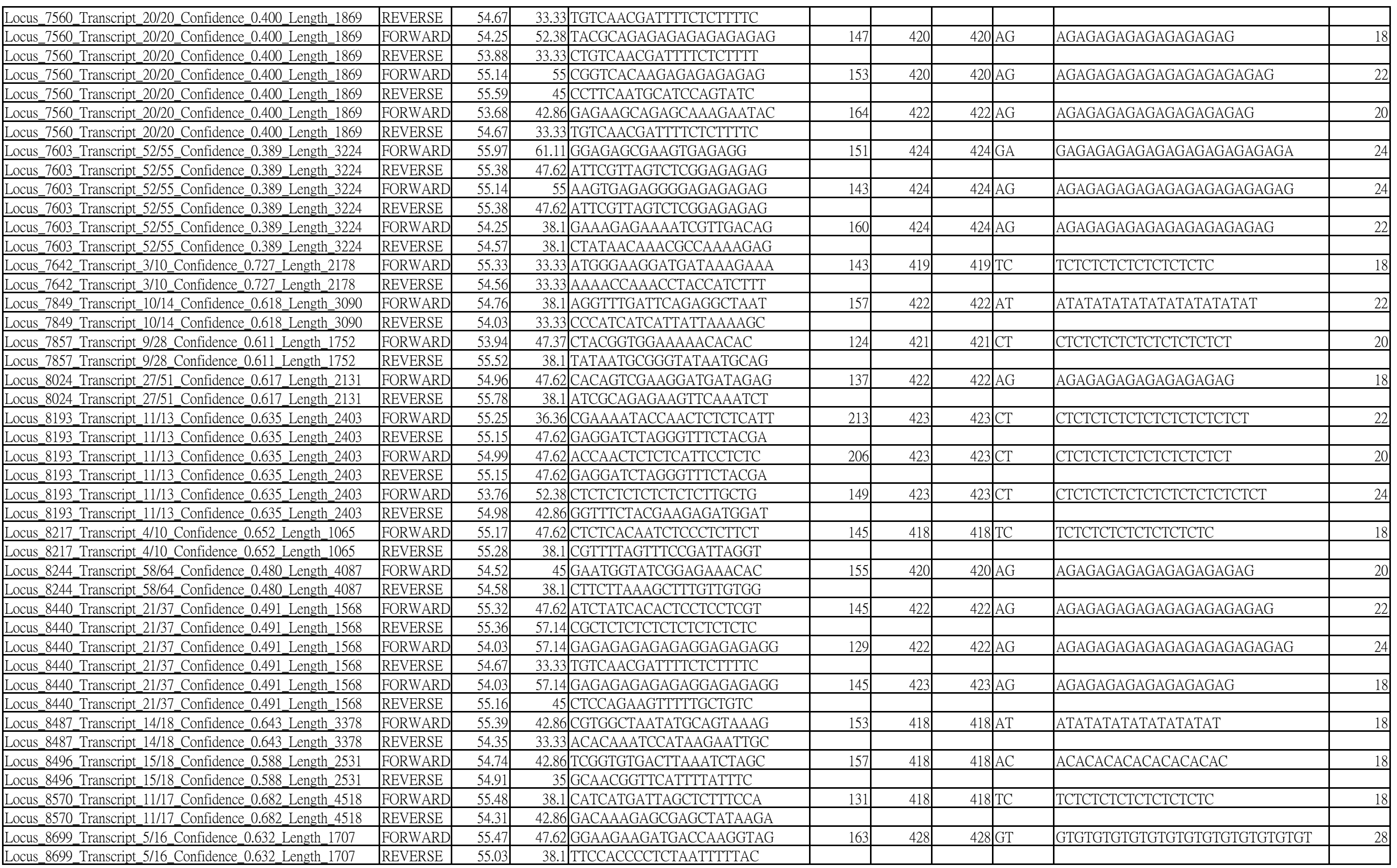


Locus_8704_Transcript_7/12_Confidence_0.615_Length_1013 Locus_8704_Transcript_7/12_Confidence_0.615_Length_1013 Locus_8710_Transcript_11/15_Confidence_0.509_Length_1052 Locus_8710_Transcript_11/15_Confidence_0.509_Length_1052 Locus_8720_Transcript_12/51_Confidence_0.558_Length_2071 Locus_8720_Transcript_12/51_Confidence_0.558_Length_2071 Locus_8762_Transcript_22/25_Confidence_0.610_Length_3370 Locus_8762_Transcript_22/25_Confidence_0.610_Length_3370 Locus_8771_Transcript_9/20_Confidence_0.504_Length_705 Locus 8771 Transcript 9/20 Confidence 0.504 Length 705 Locus 8898_Transcript_13/18_Confidence_0.625_Length_798 Locus_8898_Transcript_13/18_Confidence_0.625_Length_798 Locus_8935_Transcript_1/19_Confidence_0.488_Length_1408 Locus 8935 Transcript $1 / 19$ Confidence 0.488 Length 1408 Locus_9071_Transcript_14/15_Confidence_0.559_Length_1189 Locus_9071_Transcript_14/15_Confidence_0.559_Length_1189 Locus_9071_Transcript_14/15_Confidence_0.559_Length_1189 Locus_9071_Transcript_14/15_Confidence_0.559_Length_1189 Locus_9192_Transcript_5/16_Confidence_0.500_Length_2375 Locus_9192_Transcript_5/16_Confidence_0.500_Length_2375 Locus_9385_Transcript_5/19_Confidence_0.503_Length_1396 Locus_9385_Transcript_5/19_Confidence_0.503_Length_1396 Locus_9385_Transcript_5/19_Confidence_0.503_Length_1396 Locus_9385_Transcript_5/19_Confidence_0.503_Length_1396 Locus_9385_Transcript_5/19_Confidence_0.503_Length_1396 Locus 9385 Transcript_5/19_Confidence_0.503_Length_1396 Locus 9396 Transcript 6/9 Confidence 0.755 Length 1345 Locus_9396_Transcript_6/9_Confidence_0.755_Length_1345 Locus_9460_Transcript_17/20_Confidence_0.553_Length_1704 Locus_9460_Transcript_17/20_Confidence_0.553_Length_1704 Locus 9478 Transcript 4/12 Confidence 0.788 Length 862 Locus_9478_Transcript_4/12_Confidence_0.788_Length_862 Locus_9502_Transcript_8/19_Confidence_0.651_Length_1315 Locus_9502_Transcript_8/19_Confidence_0.651_Length_1315 Locus_9557_Transcript_13/16_Confidence_0.576_Length_1988 Locus_9557_Transcript_13/16_Confidence_0.576_Length_1988 Locus_9595_Transcript_11/19_Confidence_0.580_Length_1244 Locus_9595_Transcript_11/19_Confidence_0.580_Length_1244 Locus_9666_Transcript_26/38_Confidence_0.460_Length_1864 Locus_9666_Transcript_26/38_Confidence_0.460_Length_1864 Locus_9666_Transcript_26/38_Confidence_0.460_Length_1864 Locus_9666_Transcript 26/38 Confidence_0.460_Length_1864 Locus_9666_Transcript_26/38_Confidence_0.460_Length_1864 Locus_9666_Transcript_26/38_Confidence_0.460_Length_1864 Locus 9854 Transcript 10/22 Confidence 0.673 Length 2868

\begin{tabular}{|c|c|c|c|c|c|c|c|c|c|}
\hline FORWARD & 54.94 & 38.1 & GCAGAAGAGAATGCATAAAGA & 151 & 419 & 419 & GA & GAGAGAGAGAGAGAGAGA & 18 \\
\hline REVERSE & 55.34 & 47.37 & GCTGTGACGATTTTCCTCT & & & & & & \\
\hline FORWARD & 54.97 & 47.62 & GAGGAATCGAGAAAGAGAGAG & 167 & 423 & 423 & $\mathrm{CT}$ & СТСТСТСТСТСТСТСТСТСТСТ & 22 \\
\hline REVERSE & 55.09 & 42.86 & AGATGGTATAGGGGATTGGTA & & & & & & \\
\hline FORWARD & 54.91 & 38.1 & ATTCATCTCGGGGAATATTAG & 149 & 418 & 418 & TA & TATATATATATATATATA & 18 \\
\hline REVERSE & 54.65 & 42.86 & TAATCAAGAGATCCGTGTAGC & & & & & & \\
\hline FORWARD & 54.47 & 42.86 & TCATATACTCCATACGGCTTC & 154 & 424 & 424 & CT & СТСТСТСТСТСТСТСТСТСТСТСТ & 24 \\
\hline REVERSE & 54.31 & 33.33 & ATCAATCTTCGAATCTGCTTA & & & & & & \\
\hline FORWARD & 54.92 & 50 & TCTCTCTCTCTCTCTCTTCTGC & 138 & 424 & 424 & CT & СТСТСТСТСТСТСТСТСТСТСТСТ & 24 \\
\hline REVERSE & 54.82 & 38.1 & AATGTGTGTTTAGAGCGAAAG & & & & & & \\
\hline FORWARD & 55.58 & 50 & CGTACATTGTGAACCTCCTC & 145 & 424 & 424 & CT & СТСТСТСТСТСТСТСТСТ & 18 \\
\hline REVERSE & 54.83 & 38.1 & AAAAGATGGTATAGGGGATTG & & & & & & \\
\hline FORWARD & 56 & 57.14 & СТСТСССТССТСТСТСТСТСТ & 146 & 424 & 424 & CT & СТСТСТСТСТСТСТСТСТСТСТСТ & 24 \\
\hline REVERSE & 54.67 & 33.33 & TGTCAACGATTTTCTCTTTTC & & & & & & \\
\hline FORWARD & 54.67 & 33.33 & GAAAAGAGAAAATCGTTGACA & 156 & 424 & 424 & $\mathrm{AG}$ & AGAGAGAGAGAGAGAGAGAGAG & 22 \\
\hline REVERSE & 55.27 & 42.86 & TATGCTGCTCCCTCACTATAA & & & & & & \\
\hline FORWARD & 55.7 & 47.62 & GTAACAAGTCTTCGGAGAAGG & 138 & 419 & 419 & $\mathrm{AG}$ & AGAGAGAGAGAGAGAGAG & 18 \\
\hline REVERSE & 53.88 & 33.33 & CTGTCAACGATTTTCTCTTTT & & & & & & \\
\hline FORWARD & 55.1 & 47.62 & GAATGGAGTAGAGGGAGAAGA & 151 & 420 & 420 & $\mathrm{CT}$ & СТСТСТСТСТСТСТСТСТСТ & 20 \\
\hline REVERSE & 55.93 & 42.86 & GGAAAAGCTTCGATTACAGAG & & & & & & \\
\hline FORWARD & 55.08 & 33.33 & TCAGAAAGTGAATGAAAGGAA & 160 & 420 & 420 & GA & GAGAGAGAGAGAGAGAGAGA & 20 \\
\hline REVERSE & 53.58 & 42.86 & AAGACCTTCTCCTCTTTTCTC & & & & & & \\
\hline FORWARD & 54.16 & 52.38 & TCTCTCTCTCTCTCTCTGCTG & 150 & 424 & 424 & $\mathrm{CT}$ & СТСТСТСТСТСТСТСТСТСТСТСТ & 24 \\
\hline REVERSE & 54.77 & 45 & GTAAGGGAAACGATCATACG & & & & & & \\
\hline FORWARD & 55.03 & 38.1 & AAAATCTTCTCTTGGAACCAC & 143 & 426 & 426 & $\mathrm{CT}$ & СТСТСТСТСТСТСТСТСТСТСТСТСТ & 26 \\
\hline REVERSE & 55.54 & 47.62 & AGGAATGTAAGAGCGAGAGAG & & & & & & \\
\hline REVERSE & 53.88 & 33.33 & CTGTCAACGATTTTCTCTTTT & & & & & & \\
\hline FORWARD & 55.05 & 38.1 & GCTTTGTTTATACCTCCCATT & 165 & 422 & 422 & $\mathrm{TC}$ & TCTCTCTCTCTCTCTCTCTCTC & 22 \\
\hline
\end{tabular}




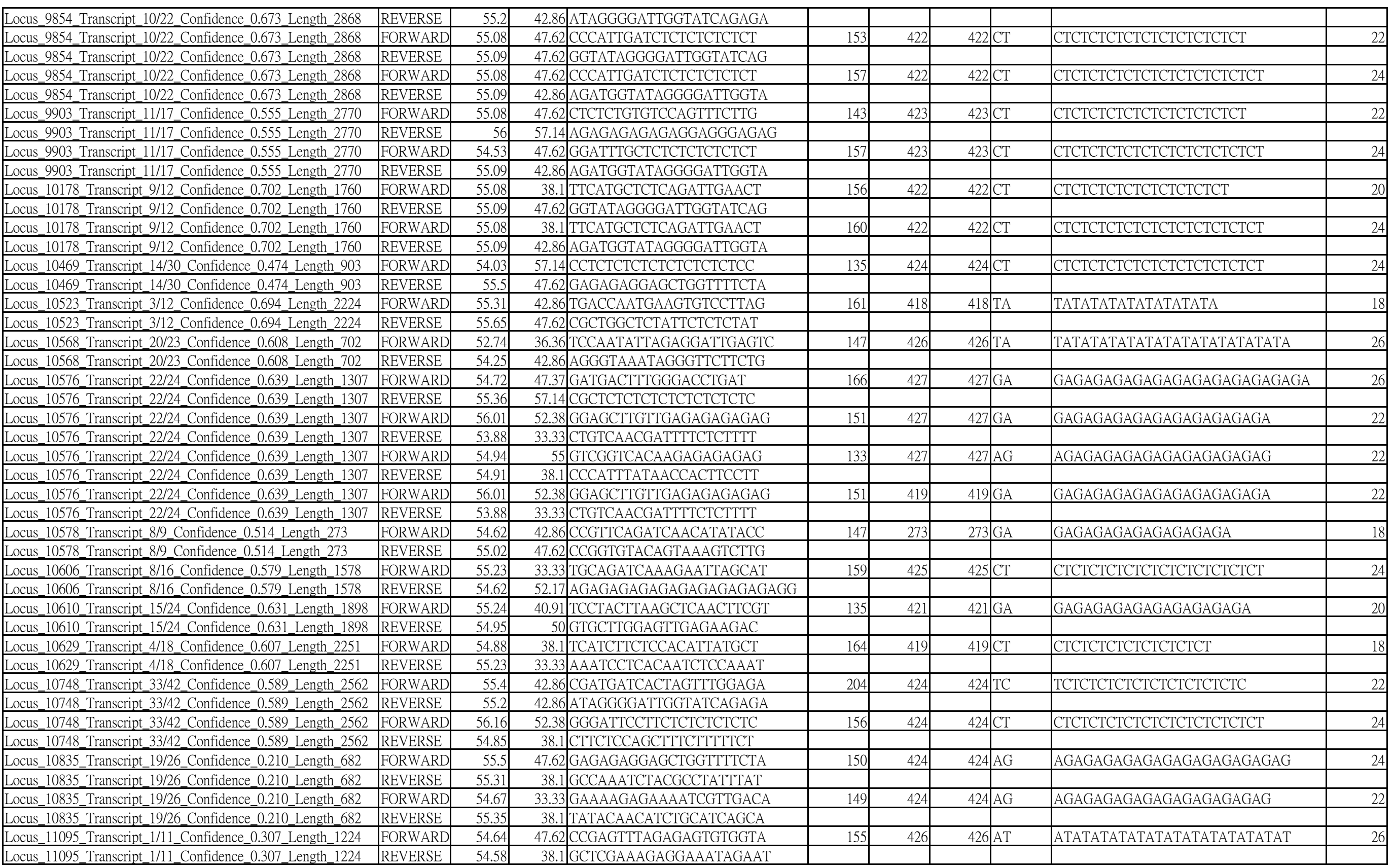


Locus_11220_Transcript_1/23_Confidence_0.573_Length_858 Locus_11220_Transcript_1/23_Confidence_0.573_Length_858 Locus_11236_Transcript_4/6_Confidence_0.720_Length_1185 Locus_11236_Transcript_4/6_Confidence_0.720_Length_1185 Locus_11288_Transcript_14/29_Confidence_0.484_Length_2142 Locus_11288_Transcript_14/29_Confidence_0.484_Length_2142 Locus_11288_Transcript_14/29_Confidence_0.484_Length_2142 Locus_11288_Transcript_14/29_Confidence_0.484_Length_2142 Locus_11288_Transcript_14/29_Confidence_0.484_Length_2142 Locus 11288 Transcript 14/29 Confidence 0.484 Length 2142 REVERSE Locus 11288 Transcript 14/29 Confidence 0.484 Length 2142 FORWARD Locus_11288_Transcript_14/29_Confidence_0.484_Length_2142 REVERSE

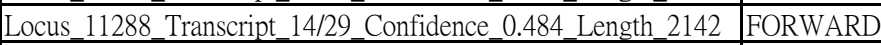
Locus_11288_Transcript_14/29_Confidence_0.484_Length_2142 REVERSE Locus_11344_Transcript_14/21_Confidence_0.531_Length_1825 FORWARD

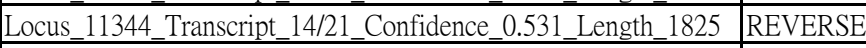

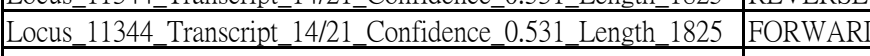
Locus 11344 Transcript 14/21 Confidence 0.531 Length 1825 REVERSE Locus_11344_Transcript_14/21_Confidence 0.531_Length_1825 FORWARD

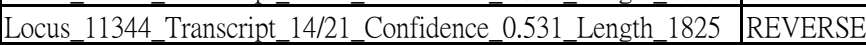
Locus_11427_Transcript_4/11_Confidence_0.667_Length_526 Locus_11427_Transcript_4/11_Confidence_0.667_Length_526 Locus_11465_Transcript_7/12_Confidence_0.811_Length_2205 Locus_11465_Transcript_7/12_Confidence_0.811_Length_2205 Locus_11465_Transcript_7/12_Confidence_0.811_Length_2205 Locus_11465_Transcript_7/12_Confidence_0.811_Length_2205 Locus 11469 Transcript 29/30 Confidence 0.612 Length 1367 Locus_11469_Transcript_29/30_Confidence_0.612_Length_1367 Locus_11469_Transcript_29/30_Confidence_0.612_Length_1367 Locus_11469_Transcript_29/30_Confidence_0.612_Length_1367 REVERSE Locus_11651_Transcript_1/9_Confidence_0.460_Length_310

Locus_11651_Transcript_1/9_Confidence_0.460_Length_310

Locus_11651_Transcript_1/9_Confidence_0.460_Length_310 Locus_11651_Transcript_1/9_Confidence_0.460_Length_310 Locus_11663_Transcript_1/11_Confidence_0.523_Length_515 Locus_11663_Transcript_1/11_Confidence_0.523_Length 5 515 Locus_11695_Transcript 5/22_Confidence_0.589_Length_71 Locus_11695_Transcript_5/22_Confidence_0.589_Length_71 Locus_12228_Transcript_3/12_Confidence_0.444_Length_433 Locus_12228 Transcript_3/12 Confidence 0.444 Length 433 Locus 12228 Transcript 3/12 Confidence 0.444 Length 433 Locus_12228_Transcript_3/12_Confidence_0.444_Length_433 Locus_12228 Transcript 3/12 Confidence 0.444 Length 433

\begin{tabular}{|c|c|c|}
\hline FORWARD & 54.99 & \begin{tabular}{l|l}
38.1 & ATTAGAAGTCTCCGTTGCTTT \\
\end{tabular} \\
\hline REVERSE & 55.06 & \begin{tabular}{l|l|}
42.86 & CTATCATTGCAGGAGAAGATG \\
\end{tabular} \\
\hline FORWARD & 55.05 & \begin{tabular}{l|l}
33.33 & GATCGTGATTTTGATGTTGTT \\
\end{tabular} \\
\hline REVERSE & 55.03 & \begin{tabular}{ll|l}
47.62 & CCGGAGACATTAGAGACCTAT \\
\end{tabular} \\
\hline FORWARD & 55 & 45.45 CTTGCCTATATACACACACCAG \\
\hline REVERSE & 54.14 & 38.1 CAGCTTGTAACCTTTTTAGGA \\
\hline FORWARD & 54.98 & 42.86 ACCCATCGAATTTACTCTCTC \\
\hline REVERSE & 55.09 & \begin{tabular}{l|l} 
42.86 & AGATGGTATAGGGGATTGGTA \\
\end{tabular} \\
\hline FORWARD & 55.09 & 42.86 TACCAATCCCCTATACCATCT \\
\hline REVERSE & 54.47 & \begin{tabular}{l|l|}
38.1 & TTATGGAAGAAGAGGAATGTG \\
\end{tabular} \\
\hline FORWARD & 55.24 & \begin{tabular}{|l|l|}
52.38 & TCTCCTCGCTCTCTACTCTCT \\
\end{tabular} \\
\hline REVERSE & 55.5 & \begin{tabular}{l|l|}
47.62 & GAGAGAGGAGCTGGTTTTCTA \\
\end{tabular} \\
\hline FORWARD & 54.85 & \begin{tabular}{l|l|}
38.1 & CGCTCTTACATTCCTTCAATA \\
\end{tabular} \\
\hline REVERSE & 55.07 & \begin{tabular}{l|l|} 
42.86 & AGGAGATGATTAGGATCGAG \\
\end{tabular} \\
\hline FORWARD & 54.59 & 42.86 CGCTAGCATCTTAATTCTCAC \\
\hline REVERSE & 54.67 & \begin{tabular}{|l|l|}
33.33 & TGTCAACGATTTTCTCTTTTC \\
\end{tabular} \\
\hline FORWARD & 54.59 & $\begin{array}{l}\text { 42.86 } \\
\text { CGCTAGCATCTTAATTCTCAC } \\
\end{array}$ \\
\hline REVERSE & 54.81 & $\begin{array}{l}\text { 47.62 } \\
\text { CAAGAGATCCCTCTCTTTCTC }\end{array}$ \\
\hline FORWARD & 54.67 & \begin{tabular}{l|l}
33.33 & GAAAAGAGAAAATCGTTGACA \\
\end{tabular} \\
\hline REVERSE & 54.85 & \begin{tabular}{l|l}
38.1 & CGATAGAAAGAATGCTGGTAA \\
\end{tabular} \\
\hline FORWARD & 54.91 & \begin{tabular}{l|l}
42.86 & CATAACCGAGTAGAGCTTTGA \\
\end{tabular} \\
\hline REVERSE & 55.14 & \begin{tabular}{|l|l|} 
50 & CTTCTGTTCCTTGCTCTGTC \\
\end{tabular} \\
\hline FORWARD & 54.51 & 47.62 GCACACACATTCTCTCTCTCT \\
\hline REVERSE & 55.09 & 47.62 GGTATAGGGGATTGGTATCAG \\
\hline FORWARD & 54.92 & 50 TTGCTCTCTCTCTCTCTCTCTC \\
\hline REVERSE & 55.09 & 42.86 AGATGGTATAGGGGATTGGTA \\
\hline FORWARD & 54.13 & 38.1 ATGAGAGATCAATCGTCAATC \\
\hline REVERSE & 54.77 & 47.62 CCGGTCCTAAATTAGTCTCTC \\
\hline FORWARD & 54.93 & \begin{tabular}{l|l}
50 & TTCGAGAGAGAGAGAGAGAGAG \\
\end{tabular} \\
\hline REVERSE & 54.83 & \begin{tabular}{l|l}
38.1 & TAATATTCTTCTACGGCATCG \\
\end{tabular} \\
\hline FORWARD & 54.22 & \begin{tabular}{l|l|}
38.1 & CTCAAAGTTGCCTCTTTTCTA \\
\end{tabular} \\
\hline REVERSE & 55.04 & \begin{tabular}{l|l|}
47.62 & GCCACTCTTCTTTCTTCTCTC \\
\end{tabular} \\
\hline FORWARD & 52.3 & \begin{tabular}{l|l}
30 & GAAAGCAAATTATTGAAGGA \\
\end{tabular} \\
\hline REVERSE & 54.73 & \begin{tabular}{l|l|}
42.86 & TCACCGCTAGGATTATAACTG \\
\end{tabular} \\
\hline FORWARD & 54.17 & 38.1 TGAAGGAATGTAAAGGAGAGA \\
\hline REVERSE & 54.73 & 42.86|TCACCGCTAGGATTATAACTG \\
\hline FORWARD & 55.5 & \begin{tabular}{|l|l|}
47.62 & GAGAGAGGGCTGGTTTTCTA \\
\end{tabular} \\
\hline REVERSE & 55.04 & 42.86 CTGATTTCTATCCCGGTACTT \\
\hline FORWARD & 55.15 & 33.33 ATATTTGCCCTTATCTTTTGG \\
\hline REVERSE & 54.48 & \begin{tabular}{l|l|}
52.38 & TCСТTCTCCTCTCTCTCTCTC \\
\end{tabular} \\
\hline FORWARD & 55.09 & \begin{tabular}{l|l|}
31.82 & GAAAGCAAATTATTGAAGGAAG \\
\end{tabular} \\
\hline REVERSE & 55.69 & \begin{tabular}{l|l|}
50 GACAGTAAGCTGACTCTCTCT \\
\end{tabular} \\
\hline FORWARD & 54.6 & \begin{tabular}{l|l|} 
52.38 & AGAGAGAGAGGAGAGAACTCG \\
\end{tabular} \\
\hline REVERSE & 54.26 & \begin{tabular}{l|l}
38.1 & TCTTTCCTTCTGAAGATCTGA \\
\end{tabular} \\
\hline FORWARD & 54.67 & \begin{tabular}{|l|l|}
33.33 & GAAAAGAGAAAATCGTTGACA \\
\end{tabular} \\
\hline
\end{tabular}

\begin{tabular}{|c|c|c|c|c|c|}
\hline 149 & 420 & 420 & CT & СТСТСТСТСТСТСТСТСТСТ & 20 \\
\hline 157 & 418 & 418 & \begin{tabular}{|l|l|} 
CT \\
\end{tabular} & СТСТСТСТСТСТСТСТСТ & 18 \\
\hline & & & & & \\
\hline 154 & 423 & 423 & CT & СТСТСТСТСТСТСТСТСТСТСТ & 22 \\
\hline 157 & 423 & 423 & \begin{tabular}{l|l} 
CT \\
\end{tabular} & \begin{tabular}{|l|} 
СТСТСТСТСТСТСТСТСТСТСТСТ \\
\end{tabular} & 24 \\
\hline & & & & & \\
\hline 166 & 424 & 424 & $\mathrm{TC}$ & TCTCТСТСТСТСТСТСТСТСТС & 22 \\
\hline & & & & & \\
\hline 153 & 423 & 423 & B СТ & СТСТСТСТСТСТСТСТСТСТСТСТ & 24 \\
\hline 156 & 423 & 423 & $3 \mathrm{TC}$ & |ТСТСТСТСТСТСТСТСТСТСТС & 22 \\
\hline & & & & & \\
\hline 118 & 424 & 424 & $\mathrm{AG}$ & AGAGAGAGAGAGAGAGAGAGAGAG & 24 \\
\hline & & & & & \\
\hline 189 & 424 & 424 & AG & AGAGAGAGAGAGAGAGAG & 18 \\
\hline & & & & & \\
\hline 148 & 424 & 424 & AG & AGAGAGAGAGAGAGAGAG & 18 \\
\hline & & & & & \\
\hline 145 & 421 & 421 & AG & AGAGAGAGAGAGAGAGAGAG & 20 \\
\hline & & & & & \\
\hline 179 & 423 & 423 & CT & СТСТСТСТСТСТСТСТСТСТСТ & 22 \\
\hline & & & & & \\
\hline 153 & 423 & 423 & CT & СТСТСТСТСТСТСТСТСТСТСТСТ & 24 \\
\hline & & & & & \\
\hline 155 & 424 & 424 & GA & GAGAGAGAGAGAGAGAGAGAGAGA & 24 \\
\hline & & & & & \\
\hline 157 & 424 & 424 & AG & AGAGAGAGAGAGAGAGAG & 18 \\
\hline & & & & & \\
\hline 157 & 419 & 419 & CT & СТСТСТСТСТСТСТСТСТ & 18 \\
\hline & & & & & \\
\hline 143 & 310 & 310 & GA & GAGAGAGAGAGAGAGAGAGAGA & 22 \\
\hline & & & & & \\
\hline 130 & 310 & 310 & AG & AGAGAGAGAGAGAGAGAG & 18 \\
\hline & & & & & \\
\hline 143 & 424 & 424 & $\mathrm{AG}$ & AGAGAGAGAGAGAGAGAGAGAGAG & 24 \\
\hline & & & & & \\
\hline 158 & 423 & 423 & B AG & AGAGAGAGAGAGAGAGAGAGAG & 22 \\
\hline & & & & & \\
\hline 149 & 423 & 423 & BA & GAGAGAGAGAGAGAGAGAGAGA & 22 \\
\hline 142 & 423 & $423+20$ & $\Delta \mathrm{G}$ & AGAGAGAGAGAGAGAGAG & 18 \\
\hline & & & & & \\
\hline 150 & 423 & 423 & AG & AGAGAGAGAGAGAGAGAGAGAG & 22 \\
\hline
\end{tabular}




\begin{tabular}{|c|c|c|c|c|c|c|c|c|c|c|}
\hline Locus_12228_Transcript_3/12_Confidence_0.444_Length_433 & REVERSE & 55.34 & 47.62 & ACCTCATAGTAGTCCCCTGAA & & & & & & \\
\hline Locus_12231_Transcript_19/22_Confidence_0.623_Length_2627 & FORWARD & 54.27 & 36.36 & TGGAGTTTCAGATGTTAGAGAA & 148 & 419 & 419 & $\mathrm{AG}$ & AGAGAGAGAGAGAGAGAG & 18 \\
\hline Locus_12231_Transcript_19/22_Confidence_0.623_Length_2627 & REVERSE & 54.44 & 38.1 & GCAGGATAATGGAATAACTCA & & & & & & \\
\hline Locus_12278_Transcript_6/14_Confidence_0.591_Length_1324 & FORWARD & 55.24 & 38.1 & TGGATCAAAGATATCATCTCG & 190 & 424 & 424 & $\mathrm{CT}$ & СТСТСТСТСТСТСТСТСТСТСТ & 22 \\
\hline Locus_12278_Transcript_6/14_Confidence_0.591_Length_1324 & REVERSE & 55.2 & 42.86 & ATAGGGGATTGGTATCAGAGA & & & & & & \\
\hline Locus_12278_Transcript_6/14_Confidence_0.591_Length_1324 & FORWARD & 55.24 & 38.1 & TGGATCAAAGATATCATCTCG & 197 & 424 & 424 & CT & СТСТСТСТСТСТСТСТСТСТСТСТ & 24 \\
\hline Locus_12278_Transcript_6/14_Confidence_0.591_Length_1324 & REVERSE & 55.09 & 42.86 & AGATGGTATAGGGGATTGGTA & & & & & & \\
\hline Locus_12348_Transcript_27/41_Confidence_0.534_Length_1122 & FORWARD & 55.17 & 38.1 & GATACGGTATAAAAGGCGAAT & 146 & 425 & 425 & $\mathrm{AG}$ & AGAGAGAGAGAGAGAGAGAGAGAG & 24 \\
\hline Locus_12348_Transcript_27/41_Confidence_0.534_Length_1122 & REVERSE & 55.1 & 31.82 & TCAACGATTTTCTCTTTTCTCT & & & & & & \\
\hline Locus_12348_Transcript_27/41_Confidence_0.534_Length_1122 & FORWARD & 56.36 & 47.62 & CGGAGAAATCAGAGAGAGAGA & 162 & 425 & 425 & $\mathrm{AG}$ & AGAGAGAGAGAGAGAGAGAGAG & 22 \\
\hline Locus_12348_Transcript_27/41_Confidence_0.534_Length_1122 & REVERSE & 54.92 & 42.86 & ATACCATATCCGCTAAGCTCT & & & & & & \\
\hline Locus_12488_Transcript_3/4_Confidence_0.875_Length_448 & FORWARD & 54.68 & 38.1 & GGATGATTTGAGGGAGATAAT & 156 & 420 & 420 & CT & СТСТСТСТСТСТСТСТСТСТСТ & 22 \\
\hline Locus_12488_Transcript_3/4_Confidence_0.875_Length_448 & REVERSE & 54.92 & 50 & GCAAGAGAGAGAGAGAGAGAGA & & & & & & \\
\hline Locus_12488_Transcript_3/4_Confidence_0.875_Length_448 & FORWARD & 55.03 & 33.33 & ATAATTGGGAGTTTTCCTTTG & 147 & 420 & 420 & CT & СТСТСТСТСТСТСТСТСТСТ & 20 \\
\hline Locus_12488_Transcript_3/4_Confidence_0.875_Length_448 & REVERSE & 64.1 & 63.16 & CTTCCGCACCGGCAGTTAG & & & & & & \\
\hline Locus_12756_Transcript_12/13_Confidence_0.725_Length_879 & FORWARD & 54.78 & 33.33 & TTTGTTTATACCTCCCATTGA & 163 & 422 & 422 & $\mathrm{TC}$ & TCTCТСТСТСТСТСТСТСТСТС & 22 \\
\hline Locus_12756_Transcript_12/13_Confidence_0.725_Length_879 & REVERSE & 55.2 & 42.86 & ATAGGGGATTGGTATCAGAGA & & & & & & \\
\hline Locus_12756_Transcript_12/13_Confidence_0.725_Length_879 & FORWARD & 55.08 & 47.62 & CCCATTGATCTCTCTCTCTCT & 157 & 422 & 422 & $\mathrm{CT}$ & СТCTCTCTCTCTCTCTCTCTCTCT & 24 \\
\hline Locus_12756_Transcript_12/13_Confidence_0.725_Length_879 & REVERSE & 55.09 & 42.86 & AGATGGTATAGGGGATTGGTA & & & & & & \\
\hline Locus_12810_Transcript_6/7_Confidence_0.674_Length_1939 & FORWARD & 54.89 & 42.86 & CCGACTACACTTGATTCAAAC & 148 & 420 & 420 & $\mathrm{CT}$ & СТСТСТСТСТСТСТСТСТСТ & 20 \\
\hline Locus_12810_Transcript_6/7_Confidence_0.674_Length_1939 & REVERSE & 54.9 & 45 & CGATCTCCAACAGTTTCTTC & & & & & & \\
\hline Locus_13064_Transcript_2/5_Confidence_0.455_Length_254 & FORWARD & 55.1 & 47.62 & CGAATAGTTAGACACGTGAGG & 110 & 254 & 254 & $\mathrm{AT}$ & ATATATATATATATATATAT & 20 \\
\hline Locus_13064_Transcript_2/5_Confidence_0.455_Length_254 & REVERSE & 55.44 & 45 & CACACACACACGCATATTCT & & & & & & \\
\hline Locus_13139_Transcript_2/3_Confidence_0.600_Length_354 & FORWARD & 55.06 & 38.1 & TCCTTCATGTAAACATTGGAC & 143 & 354 & 354 & $\mathrm{TC}$ & TСТСТСТСТСТСТСТСТСТС & 20 \\
\hline Locus_13139_Transcript_2/3_Confidence_0.600_Length_354 & REVERSE & 55.56 & 42.86 & TACCAAGTTTTGAGACAGACG & & & & & & \\
\hline Locus_13166_Transcript_2/5_Confidence_0.882_Length_621 & FORWARD & 54.85 & 38.1 & ATGCTGAAACTCTGATGTTGT & 147 & 425 & 425 & GA & GAGAGAGAGAGAGAGAGAGAGAGA & 24 \\
\hline Locus_13166_Transcript_2/5_Confidence_0.882_Length_621 & REVERSE & 55.36 & 57.14 & CGCTCTCTCTCTCTCTCTCTC & & & & & & \\
\hline Locus_13166_Transcript_2/5_Confidence_0.882_Length_621 & FORWARD & 55.02 & 57.14 & GACGGAGAGAGAGAGAGAGAG & 147 & 425 & 425 & $\mathrm{AG}$ & AGAGAGAGAGAGAGAGAGAGAGAG & 24 \\
\hline Locus_13166_Transcript_2/5_Confidence_0.882_Length_621 & REVERSE & 53.88 & 33.33 & CTGTCAACGATTTTCTCTTTT & & & & & & \\
\hline Locus_13337_Transcript_1/2_Confidence_0.750_Length_596 & FORWARD & 54.7 & 38.1 & GGAGCTTTCTTTATGTGTCAA & 170 & 421 & 421 & $\mathrm{AT}$ & ATATATATATATATATATAT & 20 \\
\hline Locus_13337_Transcript_1/2_Confidence_0.750_Length_596 & REVERSE & 55.02 & 47.62 & CGATGTCGTATAGTCCTTCTG & & & & & & \\
\hline Locus_13387_Transcript_11/12_Confidence_0.618_Length_1220 & FORWARD & 55.2 & 42.86 & CGCTTCCTTCTTTCTATTCTC & 168 & 418 & 418 & $\mathrm{TC}$ & TCTCTCTСТСТСТСТСТС & 18 \\
\hline Locus_13387_Transcript_11/12_Confidence_0.618_Length_1220 & REVERSE & 56.31 & 38.1 & TGATCTTAAAGACACCGGAAT & & & & & & \\
\hline Locus_13609_Transcript_8/11_Confidence_0.610_Length_536 & FORWARD & 56.09 & 42.11 & GAATTCACACGAGCCAAAT & 153 & 423 & 423 & CT & СТСТСТСТСТСТСТСТСТСТСТ & 22 \\
\hline Locus_13609_Transcript_8/11_Confidence_0.610_Length_536 & REVERSE & 55.09 & 42.86 & AGATGGTATAGGGGATTGGTA & & & & & & \\
\hline Locus_13778_Transcript_3/5_Confidence_0.722_Length_371 & FORWARD & 55.04 & 31.82 & AAACTGATGGATGAAATTAAGG & 149 & 371 & 371 & $\mathrm{AG}$ & AGAGAGAGAGAGAGAGAG & 18 \\
\hline Locus_13778_Transcript_3/5_Confidence_0.722_Length_371 & REVERSE & 55.32 & 47.62 & GCATTGAACCTCTCTCTCTCT & & & & & & \\
\hline Locus_13822_Transcript_9/14_Confidence_0.295_Length_323 & FORWARD & 55.22 & 42.86 & AGCATCTCTCCAAAACTAACC & 151 & 323 & 323 & $\mathrm{AG}$ & AGAGAGAGAGAGAGAGAGAGAG & 22 \\
\hline Locus_13822_Transcript_9/14_Confidence_0.295_Length_323 & REVERSE & 54.29 & 47.62 & ССТСССТАСАAТCTТСТСТСТ & & & & & & \\
\hline Locus_13822_Transcript_9/14_Confidence_0.295_Length_323 & FORWARD & 55.61 & 52.38 & AGCCGTTAGACAGAGAGAGAG & 152 & 323 & 323 & $\mathrm{AG}$ & AGAGAGAGAGAGAGAGAG & 18 \\
\hline Locus_13822_Transcript_9/14_Confidence_0.295_Length_323 & REVERSE & 54.98 & 42.86 & TGATCTGTCGTCTACCCTAAA & & & & & & \\
\hline Locus_14154_Transcript_20/27_Confidence_0.583_Length_880 & FORWARD & 54.75 & 38.1 & TGACATTGCAATATGTGTAGC & 151 & 424 & 424 & GT & GTGTGTGTGTGTGTGTGTGTGTGT & 24 \\
\hline Locus_14154_Transcript_20/27_Confidence_0.583_Length_880 & REVERSE & 55.3 & 38.1 & CСТCTCCAATTTTTCCTCTAA & & & & & & \\
\hline Locus_14543_Transcript_1/1_Confidence_0.996_Length_2557 & FORWARD & 54.97 & 38.1 & ATTGGAGATGAGCACAGTTTA & 145 & 425 & 425 & $\mathrm{AG}$ & AGAGAGAGAGAGAGAGAGAGAGAG & 24 \\
\hline Locus_14543_Transcript_1/1_Confidence_0.996_Length_2557 & REVERSE & 55.36 & 57.14 & CGCTCTCTCTCTCTCTCTCTC & & & & & & \\
\hline
\end{tabular}


Locus_14543_Transcript_1/1_Confidence_0.996_Length_2557 Locus_14543_Transcript_1/1_Confidence_0.996_Length_255 Locus_14547_Transcript_1/1_Confidence_0.971_Length_443 Locus_14547_Transcript_1/1_Confidence_0.971_Length_443 Locus_15091_Transcript_1/1_Confidence_1.000_Length_545 Locus_15091_Transcript 1/1_Confidence 1.000_Length 545 Locus_15140_Transcript_1/1_Confidence_1.000_Length_411 Locus_15140_Transcript_1/1_Confidence_1.000_Length_411 Locus_15223_Transcript_1/1_Confidence_1.000_Length_690 Locus_15223 Transcript 1/1 Confidence 1.000 Length 690 Locus_15225_Transcript_1/1_Confidence_1.000_Length_437 Locus_15225_Transcript_1/1_Confidence_1.000_Length_437 Locus_15226_Transcript_1/1_Confidence_1.000_Length_337 Locus_15226_Transcript_1/1_Confidence_1.000_Length_337 Locus_15226_Transcript_1/1_Confidence_1.000_Length_337 Locus_15226_Transcript_1/1_Confidence_1.000_Length_337 Locus_15227_Transcript_1/1_Confidence_1.000_Length_925 Locus_15227_Transcript_1/1_Confidence_1.000_Length_925 Locus_15227_Transcript_1/1_Confidence_1.000_Length_925 Locus_15227_Transcript_1/1_Confidence_1.000_Length_925 Locus_15228_Transcript_1/1_Confidence_1.000_Length_921 Locus_15228_Transcript_1/1_Confidence_1.000_Length_921 Locus_15228_Transcript_1/1_Confidence_1.000_Length_921 Locus_15228_Transcript_1/1_Confidence_1.000_Length_921 Locus_15231_Transcript_1/1_Confidence_1.000_Length_390 Locus_15231_Transcript_1/1_Confidence_1.000_Length_390 Locus 15232 Transcript $1 / 1$ Confidence 1.000 Length 421 Locus_15232_Transcript_1/1_Confidence_1.000_Length_421 Locus_15233_Transcript_1/1_Confidence_1.000_Length_382 Locus_15233_Transcript_1/1_Confidence_1.000_Length_382 Locus 15233 Transcript 1/1 Confidence 1.000 Length 382 Locus_15233_Transcript_1/1_Confidence_1.000_Length_382 Locus_15234_Transcript_1/1_Confidence_1.000_Length_415 Locus_15234_Transcript_1/1_Confidence_1.000_Length_415 Locus_15234_Transcript_1/1_Confidence_1.000_Length_415 Locus_15234_Transcript_1/1_Confidence_1.000_Length_415 Locus_15235_Transcript_1/1_Confidence_1.000_Length_415 Locus_15235_Transcript_1/1_Confidence_1.000_Length_415 Locus_15235_Transcript_1/1_Confidence_1.000_Length_415 Locus_15235_Transcript_1/1_Confidence_1.000_Length_415 Locus_15236_Transcript_1/1_Confidence_1.000_Length_448 Locus_15236_Transcript_1/1_Confidence_1.000_Length_ 448 Locus_15236_Transcript_1/1_Confidence_1.000_Length_448 Locus_15236_Transcript_1/1_Confidence_1.000_Length_448 Locus_15237_Transcript_1/1 Confidence 1.000 Length 1380
FORWARD REVERSE FORWARD REVERSE FORWARD REVERSE FORWARD REVERSE FORWAR REVERSE FORWARD REVERSE FORWARD REVERSE FORWARD REVERSE FORWARD REVERSE FORWARD REVERSE FORWARD REVERSE FORWARD REVERSE FORWARD REVERSE FORWARD REVERSE FORWARD REVERSE FORWARD REVERSE FORWARD REVERSE FORWARD REVERSE FORWARD REVERSE FORWARD REVERSE FORWARD REVERSE FORWARD REVERSE FORWARD
55 33.33 GAACATGATTTGAATTGGGTA

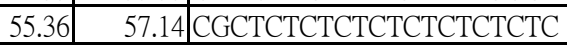
\begin{tabular}{l|l}
54.97 & 52.38 GAGAGAGAGAGAGTGGTGGAT \\
\hline
\end{tabular} \begin{tabular}{l|l|l}
54.99 & 33.33 & ACTCAAAATTGTCCTGCAATA \\
\hline
\end{tabular} \begin{tabular}{ll|l}
54.23 & 50 & CAGAAGTAGAACCAGCTTCC \\
\hline
\end{tabular} \begin{tabular}{ll|l}
55.09 & 42.86 & AGATGGTATAGGGGATTGGTA
\end{tabular} $55.66 \quad 42.86$ GGCCTTGTGTTTCTATTTAGG $\begin{array}{ll}55.35 & \text { 42.86 } \\ \text { CAAATGCTGGAAGCTATAGTG }\end{array}$ \begin{tabular}{ll|l}
55.36 & 33.33 & TAAAGGCTTTGAAAGACAACA
\end{tabular} \begin{tabular}{r|l|l}
57.98 & 55.56 & CTTTCATCCCTCCCCTTG \\
\hline
\end{tabular}

47.62 TAGAAAACCAGCTCCTCTCTC 381 AAAAGATCGTATAGGGGATTG 33.33 ACTCAAAATTGTCCTGCAATA 47.62 TGTAAGAGCGAGAGAGAGAGA 52.38 AGATCCACCACTCTCTCTCTC 33.33 GTGGATGAAAGCAAATTATTG 38.1 AACCTTTCTGAAACCTGAAAC 52.38 TAGAGAGAGAGAGGAGCTGGT 47.62 GAGGCCATAATCTCTCTCTCT 38.1 AAAAGATGGTATAGGGGATTG 38.1 AACCTTTCTGAAACCTGAAAC 47.62 GAGAGAGGAGCTGGTTTTCTA 47.62 GGCCATAATCTCTCTCTCTCT 38.1 ATAGGGGATTGGTATCAGAAA 38.1 TCCTAAAAAGGTTACAAGCTG 47.62 AGGAATGTAAGAGCGAGAGAG 38.1 TCCTAAAAAGGTTACAAGCTG 47.62 AGGAATGTAAGAGCGAGAGAG 38.1 AACCTTTCTGAAACCTGAAAC 52.38 TAGAGAGAGAGAGGAGCTGGT 47.62 GAGGCCATAATCTCTCTCTCT 38.1 AAAAGATGGTATAGGGGATTG 38.1 AACCTTTCTGAAACCTGAAAC 52.38 TAGAGAGAGAGAGGAGCTGGT 47.62 GAGGCCATAATCTCTCTCTCT 38.1 AAAAGATGGTATAGGGGATTG 38.1 AACCTTTCTGAAACCTGAAAC 47.62 GAGAGAGGAGCTGGTTTTCTA 47.62 GGCCATAATCTCTCTCTCTCT 38.1 ATAGGGGATTGGTATCAGAAA 38.1 AACCTTTCTGAAACCTGAAAC 47.62 GAGAGAGGAGCTGGTTTTCTA 47.62 GGCCATAATCTCTCTCTCTCT 38.1 ATAGGGGATTGGTATCAGAAA 38.1 TCCTAAAAAGGTTACAAGCTG

\begin{tabular}{|c|c|c|c|c|c|}
\hline 159 & 425 & 425 & $A G$ & AGAGAGAGAGAGAGAGAGAGAGAG & 24 \\
\hline & & & & & \\
\hline 145 & 419 & 419 & $\mathrm{GA}$ & GAGAGAGAGAGAGAGAGA & 18 \\
\hline 153 & 426 & 426 & TC & ТСТСТСТСТСТСТСТСТСТСТСТСТС & 26 \\
\hline & & & & & \\
\hline 158 & 411 & 411 & AT & ATATATATATATATATATATAT & 22 \\
\hline & & & & & \\
\hline 142 & 422 & 422 & $\mathrm{AG}$ & AGAGAGAGAGAGAGAGAGAGAG & 22 \\
\hline & & & & & \\
\hline 147 & 424 & 424 & $\mathrm{CT}$ & СТСТСТСТСТСТСТСТСТСТСТСТ & 24 \\
\hline & & & & & \\
\hline 155 & 337 & 337 & $\mathrm{CT}$ & СТСТСТСТСТСТСТСТСТСТСТСТ & 24 \\
\hline 150 & & 227 ا 27 & CT & 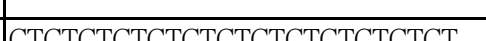 & 26 \\
\hline 150 & 351 & 331 & $\mathrm{Cl}_{1}$ & 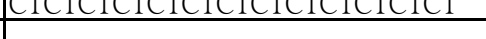 & 20 \\
\hline 155 & 423 & 423 & $\mathrm{TC}$ & TСТСТСТСТСТСТСТСТСТСТС & 22 \\
\hline & & & & & \\
\hline 148 & 423 & 423 & $\mathrm{TC}$ & 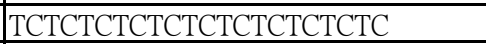 & 22 \\
\hline & & & & & \\
\hline 153 & 423 & 423 & $\mathrm{TC}$ & TCTCTСТСТСТСТСТСТСТСТС & 22 \\
\hline & & & & & \\
\hline 173 & 423 & 423 & TC & TCTCTCTCTСТСТСТСТСТСТС & 22 \\
\hline & & & & & \\
\hline 145 & 390 & 390 & CT & СТСТСТСТСТСТСТСТСТСТСТСТ & 24 \\
\hline & & & & & \\
\hline 145 & 421 & 421 & CT & СТСТСТСТСТСТСТСТСТСТСТСТ & 24 \\
\hline & & & & & \\
\hline 155 & 382 & 382 & $\mathrm{TC}$ & TCTCTСТСТСТСТСТСТСТСТС & 22 \\
\hline & & & & & \\
\hline 148 & 382 & 382 & $\mathrm{TC}$ & TCTCTCTCTCТСТСТСТСТСТС & 22 \\
\hline & & & & & \\
\hline 155 & 415 & 415 & $\mathrm{TC}$ & TCTCTCTCTCТСТСТСТСТСТС & 22 \\
\hline & & & & & \\
\hline 148 & 415 & 415 & $\mathrm{TC}$ & TCTCТСТСТСТСТСТСТСТСТС & 22 \\
\hline & & & & & \\
\hline 153 & 415 & 415 & $\mathrm{TC}$ & TСТСТСТСТСТСТСТСТСТСТС & 22 \\
\hline & & & & & \\
\hline 173 & 415 & 415 & TC & 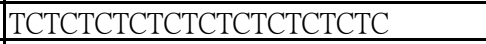 & 22 \\
\hline & & & & & \\
\hline 153 & 423 & 423 & TC & TCTCTCTCTCTCTCTCTCTCTC & 22 \\
\hline & & & & & \\
\hline 173 & 423 & 423 & TC & TCTCTCTCTСТСТСТСТСТСТС & 22 \\
\hline & & & & & \\
\hline 141 & 424 & 424 & CT & СТСТСТСТСТСТСТСТСТСТСТСТ & 24 \\
\hline
\end{tabular}




\begin{tabular}{|c|c|c|c|c|c|c|c|c|c|c|}
\hline \begin{tabular}{|l} 
Locus_15237_Transcript_1/1_Confidence_1.000_Length_1380 \\
\end{tabular} & REVERSE & 55.54 & 47.62 & AGGAATGTAAGAGCGAGAGAG & & & & & & \\
\hline Locus_15238_Transcript_1/1_Confidence_1.000_Length_619 & FORWARD & 55.29 & 40 & ACGATGAAGAAACCAAACTG & 167 & 421 & 421 & $\mathrm{TC}$ & TCTCTCTCTCTCTCTCTCTC & 20 \\
\hline Locus_15238_Transcript_1/1_Confidence_1.000_Length_619 & REVERSE & 54.69 & 47.62 & TGTAAGAGCGAGAGAGAGAGA & & & & & & \\
\hline Locus_15238_Transcript_1/1_Confidence_1.000_Length_619 & FORWARD & 55.14 & 50 & TCTCTCTCTTCTGCGCTAAC & 154 & 421 & 421 & CT & СТСТСТСТСТСТСТСТСТСТСТСТ & 24 \\
\hline Locus_15238_Transcript_1/1_Confidence_1.000_Length_619 & REVERSE & 54.99 & 35 & AGAGCGAAAGCAAATTATTG & & & & & & \\
\hline Locus_15238_Transcript_1/1_Confidence_1.000_Length_619 & FORWARD & 55.29 & 40 & ACGATGAAGAAACCAAACTG & 167 & 424 & 424 & $\mathrm{TC}$ & TСТСТСТСТСТСТСТСТСТС & 20 \\
\hline Locus_15238_Transcript_1/1_Confidence_1.000_Length_619 & REVERSE & 54.69 & 47.62 & TGTAAGAGCGAGAGAGAGAGA & & & & & & \\
\hline Locus_15239_Transcript_1/1_Confidence_1.000_Length_724 & FORWARD & 55.29 & 40 & ACGATGAAGAAACCAAACTG & 167 & 424 & 424 & $\mathrm{TC}$ & TСТСТСТСТСТСТСТСТСТС & 20 \\
\hline Locus_15239_Transcript_1/1_Confidence_1.000_Length_724 & REVERSE & 54.69 & 47.62 & TGTAAGAGCGAGAGAGAGAGA & & & & & & \\
\hline Locus_15239_Transcript_1/1_Confidence_1.000_Length_724 & FORWARD & 55.14 & 50 & TCTCTCTCTTCTGCGCTAAC & 154 & 424 & 424 & CT & СТСТСТСТСТСТСТСТСТСТСТСТ & 24 \\
\hline Locus_15239_Transcript_1/1_Confidence_1.000_Length_724 & REVERSE & 54.99 & 35 & AGAGCGAAAGCAAATTATTG & & & & & & \\
\hline Locus_15308_Transcript_1/1_Confidence_1.000_Length_522 & FORWARD & 64.39 & 66.67 & GTCGGTCGGTCGGTCACA & 145 & 419 & 419 & $A G$ & AGAGAGAGAGAGAGAGAG & 18 \\
\hline Locus_15308_Transcript_1/1_Confidence_1.000_Length_522 & REVERSE & 55.11 & 38.1 & AACCTTTCTGAAACCTGAAAC & & & & & & \\
\hline Locus_15309_Transcript_1/1_Confidence_1.000_Length_550 & FORWARD & 64.39 & 66.67 & GTCGGTCGGTCGGTCACA & 155 & 419 & 419 & $\mathrm{AG}$ & AGAGAGAGAGAGAGAGAG & 18 \\
\hline Locus_15309_Transcript_1/1_Confidence_1.000_Length_550 & REVERSE & 54.92 & 38.1 & AAGTCTTTGCTGGTAACCTTT & & & & & & \\
\hline Locus_15310_Transcript_1/1_Confidence_1.000_Length_812 & FORWARD & 64.39 & 66.67 & GTCGGTCGGTCGGTCACA & 145 & 419 & 419 & $\mathrm{AG}$ & AGAGAGAGAGAGAGAGAG & 18 \\
\hline Locus_15310_Transcript_1/1_Confidence_1.000_Length_812 & REVERSE & 55.11 & 38.1 & AACCTTTCTGAAACCTGAAAC & & & & & & \\
\hline Locus_15311_Transcript_1/1_Confidence_1.000_Length_580 & FORWARD & 64.39 & 66.67 & GTCGGTCGGTCGGTCACA & 145 & 419 & 419 & $\mathrm{AG}$ & AGAGAGAGAGAGAGAGAG & 18 \\
\hline Locus_15311_Transcript_1/1_Confidence_1.000_Length_580 & REVERSE & 55.11 & 38.1 & AACCTTTCTGAAACCTGAAAC & & & & & & \\
\hline Locus_15312_Transcript_1/1_Confidence_1.000_Length_349 & FORWARD & 64.39 & 66.67 & GTCGGTCGGTCGGTCACA & 147 & 349 & 349 & $\mathrm{AG}$ & AGAGAGAGAGAGAGAGAG & 18 \\
\hline Locus_15312_Transcript_1/1_Confidence_1.000_Length_349 & REVERSE & 54.9 & 42.86 & AGAAGTCTTTGCTGGTAACCT & & & & & & \\
\hline Locus_15313_Transcript_1/1_Confidence_1.000_Length_353 & FORWARD & 64.39 & 66.67 & GTCGGTCGGTCGGTCACA & 147 & 353 & 353 & $\mathrm{AG}$ & AGAGAGAGAGAGAGAGAG & 18 \\
\hline Locus_15313_Transcript_1/1_Confidence_1.000_Length_353 & REVERSE & 54.9 & 42.86 & AGAAGTCTTTGCTGGTAACCT & & & & & & \\
\hline Locus_15314_Transcript_1/1_Confidence_1.000_Length_308 & FORWARD & 54.72 & 47.37 & GATGACTTTGGGACCTGAT & 157 & 308 & 308 & $\mathrm{AG}$ & AGAGAGAGAGAGAGAGAGAGAG & 22 \\
\hline Locus_15314_Transcript_1/1_Confidence_1.000_Length_308 & REVERSE & 54.67 & 33.33 & TGTCAACGATTTTCTCTTTTC & & & & & & \\
\hline Locus_15314_Transcript_1/1_Confidence_1.000_Length_308 & FORWARD & 54.72 & 47.37 & GATGACTTTGGGACCTGAT & 158 & 308 & 308 & $\mathrm{AG}$ & AGAGAGAGAGAGAGAGAG & 18 \\
\hline Locus_15314_Transcript_1/1_Confidence_1.000_Length_308 & REVERSE & 53.88 & 33.33 & CTGTCAACGATTTTCTCTTTT & & & & & & \\
\hline Locus_15315_Transcript_1/1_Confidence_1.000_Length_367 & FORWARD & 54.59 & 50 & CTTCTACTTCGAGAGAGAGAGC & 109 & 367 & 367 & GA & GAGAGAGAGAGAGAGAGAGAGAGA & 24 \\
\hline Locus_15315_Transcript_1/1_Confidence_1.000_Length_367 & REVERSE & 54.67 & 33.33 & TGTCAACGATTTTCTCTTTTC & & & & & & \\
\hline Locus_15315_Transcript_1/1_Confidence_1.000_Length_367 & FORWARD & 55.36 & 57.14 & GCGAGAGAGAGAGAGAGAGAG & 152 & 367 & 367 & $\mathrm{AG}$ & AGAGAGAGAGAGAGAGAG & 18 \\
\hline Locus_15315_Transcript_1/1_Confidence_1.000_Length_367 & REVERSE & 54.61 & 47.37 & ATCTGGATCACCAGCTTCT & & & & & & \\
\hline Locus_15315_Transcript_1/1_Confidence_1.000_Length_367 & FORWARD & 54.67 & 33.33 & GAAAAGAGAAAATCGTTGACA & 152 & 367 & 367 & $\mathrm{AG}$ & AGAGAGAGAGAGAGAGAGAGAG & 22 \\
\hline Locus_15315_Transcript_1/1_Confidence_1.000_Length_367 & REVERSE & 54.69 & 47.37 & ATGATGGTGGTGGTGTATG & & & & & & \\
\hline Locus_15316_Transcript_1/1_Confidence_1.000_Length_1355 & FORWARD & 55.05 & 57.89 & GTATAGACGCGGAGGAGAG & 180 & 424 & 424 & GA & GAGAGAGAGAGAGAGAGAGAGAGA & 24 \\
\hline Locus_15316_Transcript_1/1_Confidence_1.000_Length_1355 & REVERSE & 53.88 & 33.33 & CTGTCAACGATTTTCTCTTTT & & & & & & \\
\hline Locus_15316_Transcript_1/1_Confidence_1.000_Length_1355 & FORWARD & 55.36 & 57.14 & GCGAGAGAGAGAGAGAGAGAG & 156 & 424 & 424 & $A G$ & AGAGAGAGAGAGAGAGAG & 18 \\
\hline Locus_15316_Transcript_1/1_Confidence_1.000_Length_1355 & REVERSE & 54.69 & 50 & ATCACCATGGATCACCAG & & & & & & \\
\hline Locus_15316_Transcript_1/1_Confidence_1.000_Length_1355 & FORWARD & 54.67 & 33.33 & GAAAAGAGAAAATCGTTGACA & 180 & 424 & 424 & $\mathrm{AG}$ & AGAGAGAGAGAGAGAGAGAGAG & 22 \\
\hline Locus_15316_Transcript_1/1_Confidence_1.000_Length_1355 & REVERSE & 54.97 & 47.37 & CAGGCTGATATGCTTCGTA & & & & & & \\
\hline Locus_15317_Transcript_1/1_Confidence_1.000_Length_529 & FORWARD & 54.25 & 38.1 & GAAAGAGAAAATCGTTGACAG & 175 & 422 & 422 & $\mathrm{AG}$ & AGAGAGAGAGAGAGAGAGAGAG & 22 \\
\hline Locus_15317_Transcript_1/1_Confidence_1.000_Length_529 & REVERSE & 54.8 & 47.62 & СTGCTCTTTTCTCTCСTCTCT & & & & & & \\
\hline Locus_15317_Transcript_1/1_Confidence_1.000_Length_529 & FORWARD & 54.67 & 33.33 & GAAAAGAGAAAATCGTTGACA & 161 & 422 & 422 & $\mathrm{AG}$ & AGAGAGAGAGAGAGAGAGAGAG & 22 \\
\hline Locus_15317_Transcript_1/1_Confidence_1.000_Length_529 & REVERSE & 54.85 & 42.86 & GCTGATATGGTGATATGCTTC & & & & & & \\
\hline Locus_15318_Transcript_1/1_Confidence_1.000_Length_228 & FORWARD & 55.8 & 52.38 & GAGCGAGAGAGAGAGAGAGAA & 118 & 228 & 228 & $\mathrm{AG}$ & AGAGAGAGAGAGAGAGAG & 18 \\
\hline Locus_15318_Transcript_1/1_Confidence_1.000_Length_228 & REVERSE & 54.04 & 55.56 & CCACTGCTCTTCGCTAGT & & & & & & \\
\hline
\end{tabular}


Locus_15319_Transcript_1/1_Confidence_1.000_Length_539 Locus_15319_Transcript_1/1_Confidence_1.000_Length_539 Locus_15319_Transcript_1/1_Confidence_1.000_Length_539 Locus_15319_Transcript_1/1_Confidence_1.000_Length_539 Locus_15319_Transcript_1/1_Confidence_1.000_Length_539 Locus_15319_Transcript_1/1_Confidence_1.000_Length 539 Locus_15320_Transcript_1/1_Confidence_1.000_Length_701 Locus_15320_Transcript_1/1_Confidence_1.000_Length_701 Locus_15320_Transcript_1/1_Confidence_1.000_Length_701 Locus_15320_Transcript_1/1_Confidence_1.000_Length_70 Locus_15321_Transcript_1/1_Confidence_1.000_Length_524 Locus_15321_Transcript_1/1_Confidence_1.000_Length_524 Locus_15321_Transcript_1/1_Confidence_1.000_Length_524 Locus_15321_Transcript_1/1_Confidence_1.000_Length_524 Locus_15322_Transcript_1/1_Confidence_1.000_Length_368 Locus_15322_Transcript_1/1_Confidence_1.000_Length_368 Locus_15323_Transcript_1/1_Confidence_1.000_Length_518 Locus_15323_Transcript_1/1_Confidence_1.000_Length_518 Locus_15324_Transcript_1/1_Confidence_1.000_Length_335 Locus_15324_Transcript_1/1_Confidence_1.000_Length_335 Locus_15325_Transcript_1/1_Confidence_1.000_Length_302 Locus_15325_Transcript_1/1_Confidence_1.000_Length_302 Locus_15326_Transcript_1/1_Confidence_1.000_Length_650 Locus_15326_Transcript_1/1_Confidence_1.000_Length_650 Locus_15897_Transcript_1/1_Confidence_1.000_Length_804 Locus_15897_Transcript_1/1_Confidence_1.000_Length_804 Locus 15898 Transcript $1 / 1$ Confidence 1.000 Length 803 Locus_15898_Transcript_1/1_Confidence_1.000_Length_803 Locus_15899_Transcript_1/1_Confidence_1.000_Length_804 Locus_15899_Transcript_1/1_Confidence_1.000 Length_804 Locus 15900 Transcript $1 / 1$ Confidence 1.000 Length 831 Locus_15900_Transcript_1/1_Confidence_1.000_Length_831 Locus_16624_Transcript_1/1_Confidence_1.000_Length_472 Locus_16624_Transcript_1/1_Confidence_1.000_Length_472 Locus 16625 Transcript 1/1 Confidence 1.000 Length 43 Locus 16625 Transcript $1 / 1$ Confidence 1.000 Length 431 Locus 16626 Transcript 1/1 Confidence 1.000 Length 397 Locus_16626_Transcript_1/1_Confidence_1.000_Length_397 Locus_16627_Transcript_1/1_Confidence_1.000_Length_399 Locus_16627_Transcript_1/1_Confidence_1.000_Length_399 Locus_16628_Transcript_1/1_Confidence_1.000_Length_305 Locus_16628_Transcript_1/1_Confidence_1.000_Length_305 Locus_16644_Transcript_1/1_Confidence_1.000_Length_426 Locus 16644 Transcript $1 / 1$ Confidence 1.000 Length 426 Locus_16645_Transcript_1/1_Confidence_1.000_Length_424 \begin{tabular}{|r|r|r|l|}
\hline FORWARD & 54.94 & 52.38 & AGCGAGAGAGAGAGAGAGAAG \\
\hline
\end{tabular} REVERSE

FORWAR REVERSE FORWARD REVERSE FORWARD REVERSE FORWARD REVERSE FORWARD REVERSE FORWARD REVERSE FORWARD REVERSE FORWARD REVERSE FORWARD REVERSE FORWARD REVERSE FORWARD REVERSE FORWARD REVERSE FORWARD REVERSE FORWARD REVERSE FORWAR REVERSE FORWARD REVERSE FORWARD REVERSE FORWARD REVERSE FORWARD REVERSE FORWARD REVERSE FORWARD REVERSE FORWARD 52.63 CTCTTGTGACCGATCCTCT

33.33 GAAAAGAGAAAATCGTTGACA

52.38 CTGCTCTTCTCTCCTCTCTCT

33.33 GAAAAGAGAAAATCGTTGACA 45 GCTGATATGGATGCTTCGTA 30.43 AAGTAAGTAAAAGATGGTATTCG 57.14 CTGCTCCTCTCTCTCTCTCTC 33.33 GAAAAGAGAAAATCGTTGACA 33.33 TGTTTTTAGCCATAACCTTGA 57.89 GGAGCAGGAGAGGTAGTTG 33.33 TGTCAACGATTTTCTCTTTTC 57.89 GGAGCAGGAGAGGTAGTTG 33.33 CTGTCAACGATTTTCTCTTTT 42.86 AGATGGTATAGGGGATTGGTA 38.1 AAAGATTCTGAAACGAGGACT 38.1 GAGGTGAAAGAGAAAATCGTT 50 CGAGAAAGTCCCATACTGAG 47.62 GGTATAGGGGATTGGTATCAG 42.86 CAGTCAAACACCCACTAATGT 47.62 GGTATAGGGGATTGGTATCAG 42.86 CAGTCAAACACCCACTAATGT 38.1 CAAAGAGAAAATCGTTGACAG 45 GAACACAGCATGGTTCTTCT 38.1 GCCTCTGTTGTCACAAAATTA 42.86 AAAGTGTTCTAAAGGGGAGTG 42.86 TTAGTTGGTCAGTCTGGACAT 38.1 GAAGGGGGATAATTAAAAGTG 42.86 TTAGTTGGTCAGTCTGGACAT 38.1 GAAGGGGGATAATTAAAAGTG 42.86 TTAGTTGGTCAGTCTGGACAT 42.86 AAAGTGTTCTAAAGGGGAGTG 42.86 TATCACATATGACCCCTGTTC 33.33 GAGATTGAATTTCATGTTGGT

\begin{tabular}{r|r|r}
54.1 & 33.33 & \\
54.85 & 4286 & TATCACATATGACCCCTCTTC
\end{tabular} 54.8542 .86 TATCACATATGACCCCTGTTC

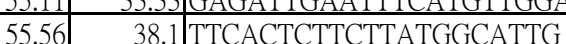
33.33 GAGATTGAATTTCATGTTGGA 38.1 TTCACTCTTCTTATGGCATTG 33.33 GAGATTGAATTTCATGTTGGA 33.33 CAGGCAAAACAAGT 38.1 CACACACAACACACACATTTI 38.1 CACACACAACACACACATTTT 47.62 AGAAGGAAGATCTGCGAGTAG 47.62 AGCGTAGAACCCTACAGAATC 47.62 AGAAGGAAGATCTGCGAGTAG 


\begin{tabular}{|c|c|c|c|c|c|c|c|c|c|c|}
\hline Locus_16645_Transcript_1/1_Confidence_1.000_Length_424 & REVERSE & 55.25 & 47.62 & AGCGTAGAACCCTACAGAATC & & & & & & \\
\hline Locus_16903_Transcript_1/1_Confidence_1.000_Length_551 & FORWARD & 54.99 & 33.33 & ACTCAAAATTGTCCTGCAATA & 145 & 419 & 419 & CT & СТСТСТСТСТСТСТСТСТ & 18 \\
\hline Locus_16903_Transcript_1/1_Confidence_1.000_Length_551 & REVERSE & 54.97 & 52.38 & GAGAGAGAGAGAGTGGTGGAT & & & & & & \\
\hline Locus_16904_Transcript_1/1_Confidence_1.000_Length_284 & FORWARD & 54.99 & 33.33 & ACTCAAAATTGTCCTGCAATA & 145 & 284 & 284 & CT & СТСТСТСТСТСТСТСТСТ & 18 \\
\hline Locus_16904_Transcript_1/1_Confidence_1.000_Length_284 & REVERSE & 54.97 & 52.38 & GAGAGAGAGAGAGTGGTGGAT & & & & & & \\
\hline Locus_16905_Transcript_1/1_Confidence_1.000_Length_250 & FORWARD & 54.99 & 33.33 & ACTCAAAATTGTCCTGCAATA & 145 & 250 & 250 & CT & СТСТСТСТСТСТСТСТСТ & 18 \\
\hline Locus_16905_Transcript_1/1_Confidence_1.000_Length_250 & REVERSE & 54.97 & 52.38 & GAGAGAGAGAGAGTGGTGGAT & & & & & & \\
\hline Locus_17089_Transcript_1/1_Confidence_1.000_Length_298 & FORWARD & 55.08 & 38.1 & TGACACAACGAAGAAGAAGTT & 141 & 298 & 298 & $\mathrm{AG}$ & AGAGAGAGAGAGAGAGAGAG & 20 \\
\hline Locus_17089_Transcript_1/1_Confidence_1.000_Length_298 & REVERSE & 54.5 & 42.86 & ATGTTCTCTTCTCCTCCATCT & & & & & & \\
\hline Locus_17313_Transcript_1/1_Confidence_1.000_Length_334 & FORWARD & 54.28 & 33.33 & AAAACTCAACATTTGGTTCAC & 150 & 334 & 334 & AT & ATATATATATATATATATATAT & 22 \\
\hline Locus_17313_Transcript_1/1_Confidence_1.000_Length_334 & REVERSE & 52.61 & 33.33 & TAAGGTCAGTTTGAGATTGAA & & & & & & \\
\hline Locus_19085_Transcript_1/1_Confidence_1.000_Length_316 & FORWARD & 52.61 & 33.33 & TAAGGTCAGTTTGAGATTGAA & 151 & 316 & 316 & $\mathrm{AT}$ & ATATATATATATATATATATAT & 22 \\
\hline Locus_19085_Transcript_1/1_Confidence_1.000_Length_316 & REVERSE & 55.71 & 33.33 & CAGGCAAAACAAGTTAAACAA & & & & & & \\
\hline Locus_19086_Transcript_1/1_Confidence_1.000_Length_336 & FORWARD & 52.61 & 33.33 & TAAGGTCAGTTTGAGATTGAA & 152 & 336 & 336 & $\mathrm{AT}$ & ATATATATATATATATATATAT & 22 \\
\hline Locus_19086_Transcript_1/1_Confidence_1.000_Length_336 & REVERSE & 54.28 & 33.33 & AAAACTCAACATTTGGTTCAC & & & & & & \\
\hline Locus_19086_Transcript_1/1_Confidence_1.000_Length_336 & FORWARD & 55.11 & 33.33 & GAGATTGAATTTCATGTTGGA & 159 & 336 & 336 & $\mathrm{TA}$ & TATATATATATATATATA & 18 \\
\hline Locus_19086_Transcript_1/1_Confidence_1.000_Length_336 & REVERSE & 55.71 & 33.33 & CAGGCAAAACAAGTTAAACAA & & & & & & \\
\hline Locus_19087_Transcript_1/1_Confidence_1.000_Length_314 & FORWARD & 52.61 & 33.33 & TAAGGTCAGTTTGAGATTGAA & 149 & 314 & 314 & AT & ATATATATATATATATATAT & 20 \\
\hline Locus_19087_Transcript_1/1_Confidence_1.000_Length_314 & REVERSE & 55.71 & 33.33 & CAGGCAAAACAAGTTAAACAA & & & & & & \\
\hline Locus_19088_Transcript_1/1_Confidence_1.000_Length_318 & FORWARD & 55.11 & 33.33 & GAGATTGAATTTCATGTTGGA & 141 & 318 & 318 & AT & ATATATATATATATATATATAT & 22 \\
\hline Locus_19088_Transcript_1/1_Confidence_1.000_Length_318 & REVERSE & 55.71 & 33.33 & CAGGCAAAACAAGTTAAACAA & & & & & & \\
\hline Locus_21552_Transcript_1/1_Confidence_1.000_Length_249 & FORWARD & 53.94 & 47.37 & CTACGGTGGAAAAACACAC & 176 & 249 & 249 & $\mathrm{CT}$ & СТCTCTCTCTCTCTCTCTCT & 20 \\
\hline Locus_21552_Transcript_1/1_Confidence_1.000_Length_249 & REVERSE & 53.91 & 38.1 & GGATTATCCAAAGAGTTGTTG & & & & & & \\
\hline Locus_21553_Transcript_1/1_Confidence_1.000_Length_323 & FORWARD & 53.94 & 47.37 & CTACGGTGGAAAAACACAC & 124 & 323 & 323 & $\mathrm{CT}$ & СТСТCТСТСТСТСТСТСТСТ & 20 \\
\hline Locus_21553_Transcript_1/1_Confidence_1.000_Length_323 & REVERSE & 55.52 & 38.1 & TATAATGCGGGTATAATGCAG & & & & & & \\
\hline Locus_21555_Transcript_1/1_Confidence_1.000_Length_230 & FORWARD & 53.94 & 47.37 & CTACGGTGGAAAAACACAC & 169 & 230 & 230 & $\mathrm{CT}$ & СТСТCTСТСТСТСТСТСТСТ & 20 \\
\hline Locus_21555_Transcript_1/1_Confidence_1.000_Length_230 & REVERSE & 55.39 & 38.1 & TTATCCAAAGAGTTGTGATGC & & & & & & \\
\hline
\end{tabular}




\begin{tabular}{|c|c|c|c|c|c|c|c|c|}
\hline Seq ID & Orientation & $\operatorname{tm}$ & $\mathrm{GC} \%$ & Seq & Prod size & Motif & SSR & SSRLen \\
\hline Locus_25_Transcript_59/302_Confidence_0.084_Length_2575 & FORWARD & 54.9 & 40.91 & CTGGTAGATGGATCTATTTGGT & 139 & TTC & TTCTTCTTCTTCTTCTTCTTCTTC & 2 \\
\hline Locus_25_Transcript_59/302_Confidence_0.084_Length_2575 & REVERSE & 54.98 & 36.36 & GAAGAAGAAGAAGAAGAAGCAA & & & & \\
\hline Locus_27_Transcript_125/143_Confidence_0.269_Length_5400 & FORWARD & 54.81 & 36.36 & CGTGTTGTGAAGAAAGATCTAA & 153 & TGA & TGATGATGATGATGATGATGATGA & 24 \\
\hline Locus_27_Transcript_125/143_Confidence_0.269_Length_5400 & REVERSE & 54.95 & 38.1 & TAACTTCCTCCAACTCATCAA & & & & \\
\hline Locus_29_Transcript_121/307_Confidence_0.050_Length_3068 & FORWARD & 56.4 & 52.63 & GAAAGCTCGAGAGCAACAAC & 264 & ACA & ACAACAACAACAACAACAACAACAACAA & 3 \\
\hline Locus_29_Transcript_121/307_Confidence_0.050_Length_3068 & REVERSE & 57.64 & 55.56 & GCTGTGGGAGTATTTTGTCG & & & & \\
\hline Locus_36_Transcript_19/23_Confidence_0.589_Length_956 & FORWARD & 54.62 & 28.57 & CAATAAGGTTTGGTTGAAAAA & 152 & GAT & GATGATGATGATGATGATGAT & 21 \\
\hline Locus_36_Transcript_19/23_Confidence_0.589_Length_956 & REVERSE & 55.08 & 38.1 & GGAGCTTTATTGATCTTCCAT & & & & \\
\hline Locus_163_Transcript_23/42_Confidence_0.552_Length_2405 & REVERSE & 54.63 & 42.86 & ССТTCTТССТТСТССТTCTТА & & & & \\
\hline Locus_168_Transcript_15/35_Confidence_0.595_Length_2203 & FORWARD & 54.34 & 52.38 & AGAGAGAGAGGGAGAGAGATG & 114 & GAG & GAGGAGGAGGAGGAGGAGGAG & 2. \\
\hline Locus_168_Transcript_15/35_Confidence_0.595_Length_2203 & REVERSE & 54.77 & 38.1 & CTTCTTTGATTCAATCCACAC & & & & \\
\hline Locus_174_Transcript_3/31_Confidence_0.373_Length_3861 & FORWARD & 54.04 & 50 & GTACAAGCTGGGAGTTCTTC & 167 & GAG & GAGGAGGAGGAGGAGGAG & 18 \\
\hline Locus_174_Transcript_3/31_Confidence_0.373_Length_3861 & REVERSE & 54.83 & 38.1 & AATGAGAAGAAAAGGAGGAGA & & & & \\
\hline Locus_177_Transcript_49/62_Confidence_0.280_Length_2285 & FORWARD & 54.54 & 42.86 & ACTCACTCTGAAAATGCTCAC & 147 & TCT & TCTTCT & 3 \\
\hline Locus_177_Transcript_49/62_Confidence_0.280_Length_2285 & REVERSE & 55.28 & 38.1 & TTAGAGGAGATGCAAACAAGA & & & & \\
\hline Locus_177_Transcript_49/62_Confidence_0.280_Length_2285 & FORWARD & 54.54 & 42.86 & ACTCACTCTGAAAATGCTCAC & 149 & TTC & TTCTTCTTCTTCTTCTTCTTCTTC & 2 \\
\hline Locus_178_Transcript_7/13_Confidence_0.627_Length_1991 & REVERSE & 54.97 & 42.86 & AGCCTCTCACGACTTATTTCT & & & & \\
\hline Locus_206_Transcript_69/94_Confidence_0.185_Length_7868 & FORWARD & 55.39 & 47.62 & CCCCCTATGTATATCAAGAGC & 170 & AGA & GAAGAAGAAGAAGA & 18 \\
\hline Locus_206_Transcript_69/94_Confidence_0.185_Length_7868 & REVERSE & 54.88 & 38.1 & CTCAAGCCTATTTGTTCTCAA & & & & \\
\hline Locus_252_Transcript_33/65_Confidence_0.587_Length_5610 & FORWARD & 55.26 & 42.86 & GTGATACAGGCCAATCTTGTA & 151 & GCA & GCAGCAGCAGCAGCAGCA & 18 \\
\hline Locus_252_Transcript_33/65_Confidence_0.587_Length_5610 & REVERSE & 55.03 & 40 & CTGATTTTGGTGTTGGATCT & & & & \\
\hline Locus_262_Transcript_104/104_Confidence_0.386_Length_4827 & FORWARD & 54.94 & 33.33 & GTATTTCAATTCCCGAAATCT & 150 & $\mathrm{AAG}$ & AAGAAGAAGAAGAAGAAG & 18 \\
\hline Locus_262_Transcript_104/104_Confidence_0.386_Length_4827 & REVERSE & 54.87 & 42.86 & TTCTTCTTCTGCTGTGACTTC & & & & \\
\hline Locus_293_Transcript_30/33_Confidence_0.617_Length_6683 & FORWARD & 55.04 & 38.1 & AACAACAACAACATCAACCTC & 142 & $\mathrm{AAC}$ & AACAACAACAACAACAAC & 18 \\
\hline Locus_293_Transcript_30/33_Confidence_0.617_Length_6683 & REVERSE & 54.53 & 33.33 & AAAAAGATTCATGCAACTGAC & & & & \\
\hline Locus_293_Transcript_30/33_Confidence_0.617_Length_6683 & FORWARD & 55.3 & 42.86 & GAGCAGCTGTATTTTGAGATG & 154 & TCA & CATCATCATCATCATCATCA & 2 \\
\hline Locus_293_Transcript_30/33_Confidence_0.617_Length_6683 & REVERSE & 56.03 & 33.33 & ATCAATTGCATGAATCAGATG & & & & \\
\hline Locus_317_Transcript_60/81_Confidence_0.581_Length_4655 & FORWARD & 54.6 & 42.86 & TCTTCATCGGAGTCTATCTTG & 144 & ATG & ATGATGATGATGATGATGATG & 2 \\
\hline Locus_317_Transcript_60/81_Confidence_0.581_Length_4655 & REVERSE & 55.31 & 38.1 & ACCCTAGCCAAAATACATCAT & & & & \\
\hline Locus_372_Transcript_29/37_Confidence_0.298_Length_1455 & FORWARD & 57.14 & 45 & ACCCAAAAGCTCTCCTCTTT & 151 & CTG & CTGCTGCTGCTGCTGCTG & 18 \\
\hline Locus_372_Transcript_29/37_Confidence_0.298_Length_1455 & REVERSE & 54.85 & 42.86 & TGCACATAAGTCAGACAAGTG & & & & \\
\hline Locus_418_Transcript_33/61_Confidence_0.206_Length_1216 & FORWARD & 55.16 & 42.86 & ACTATTAACAAGGGCGTAACC & 128 & ATA & ATAATAATAATAATAATAATA & 2. \\
\hline Locus_418_Transcript_33/61_Confidence_0.206_Length_1216 & REVERSE & 54.57 & 33.33 & TAAGTAATTTTGTCCCAATCG & & & & \\
\hline
\end{tabular}




\begin{tabular}{|c|c|c|c|c|c|c|c|}
\hline Locus_448_Transcript_388/463_Confidence_0.042_Length_4620 & FORWARD & 55.09 & \begin{tabular}{l|l}
38.1 & CGTATCTTAAGTCGCAGAAAA \\
\end{tabular} & 155 & $\mathrm{TCC}$ & TCСТCСТССТССТССТСC & 18 \\
\hline Locus_448_Transcript_388/463_Confidence_0.042_Length_4620 & REVERSE & 55.12 & 47.62 CTTTAGACGAGGAAGAGGAAG & & & & \\
\hline Locus_463_Transcript_26/45_Confidence_0.435_Length_1525 & FORWARD & 54.92 & 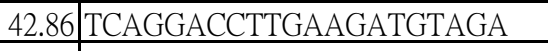 & 150 & CTT & CTTCTTCTTCTTCTTCTT & 18 \\
\hline Locus_463_Transcript_26/45_Confidence_0.435_Length_1525 & REVERSE & 55.17 & 35 AAAAGAAACCAGTTGCAGAA & & & & \\
\hline Locus_470_Transcript_37/42_Confidence_0.474_Length_1552 & FORWARD & 53 & \begin{tabular}{l|l}
28.57 & CATCCAAACATTAACTTTTCA \\
\end{tabular} & 145 & AAT & AATAATAATAATAATAATAAT & 21 \\
\hline Locus_470_Transcript_37/42_Confidence_0.474_Length_1552 & REVERSE & 54.99 & \begin{tabular}{l|l}
40.91 & GTATTGACTGTTTTGAGTGTCG \\
\end{tabular} & & & & \\
\hline Locus_504_Transcript_8/30_Confidence_0.623_Length_1266 & FORWARD & 54.71 & \begin{tabular}{l|l}
40 & GAATTAATGAGATCGCTGCT \\
\end{tabular} & 150 & GAA & GAAGAAGAAGAAGAAGAA & 18 \\
\hline Locus_504_Transcript_8/30_Confidence_0.623_Length_1266 & REVERSE & 54.47 & 33.33 TCGGTAAATCGACATTTAATC & & & & \\
\hline Locus_516_Transcript_115/164_Confidence_0.244_Length_9547 & FORWARD & 54.39 & 31.82 AACATCAATAGGGAAAATAAGC & 152 & TGC & TGCTGCTGCTGCTGCTGCTGCTGC & 24 \\
\hline Locus_516_Transcript_115/164_Confidence_0.244_Length_9547 & REVERSE & 55.02 & $\begin{array}{l}42.86 \\
\text { GACATAGCCTACATGAAAACG } \\
\end{array}$ & & & & \\
\hline Locus_559_Transcript_12/14_Confidence_0.669_Length_1496 & FORWARD & 54.51 & 38.1 TGAGAGCGAGAAATATAGCAT & 155 & TTG & TTGTTGTTGTTGTTGTTGTTGTTGTTG & 27 \\
\hline Locus_559_Transcript_12/14_Confidence_0.669_Length_1496 & REVERSE & 54.94 & \begin{tabular}{l|l}
33.33 & ACAACAACAACAACAACAACA \\
\end{tabular} & & & & \\
\hline Locus_559_Transcript_12/14_Confidence_0.669_Length_1496 & FORWARD & 54.94 & 33.33 TGTTGTTGTTGTTGTTGTTGT & 150 & TTG & TTGTTGTTGTTGTTGTTGTTGTTGTTG & 27 \\
\hline Locus_559_Transcript_12/14_Confidence_0.669_Length_1496 & REVERSE & 54.57 & \begin{tabular}{l|l|}
33.33 & CATAAATACGCCGAACATAAT \\
\end{tabular} & & & & \\
\hline Locus_593_Transcript_18/25_Confidence_0.574_Length_2205 & FORWARD & 54.98 & 33.33 TGATATGGATGGTAAAAATGG & 147 & GGC & GGCGGCGGCGGCGGCGGCGGC & 21 \\
\hline Locus_593_Transcript_18/25_Confidence_0.574_Length_2205 & REVERSE & 55.3 & 42.86 TCTCCACACTCGTAACATTTC & & & & \\
\hline Locus_609_Transcript_20/23_Confidence_0.590_Length_1511 & FORWARD & 54.93 & 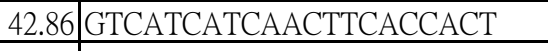 & 162 & $\mathrm{GCC}$ & GCCGCCGCCGCCGCCGCC & 18 \\
\hline Locus_609_Transcript_20/23_Confidence_0.590_Length_1511 & REVERSE & 54.91 & 28.57 TGAAACCAATTGGAATCTAAA & & & & \\
\hline Locus_615_Transcript_28/58_Confidence_0.419_Length_1952 & FORWARD & 54.9 & \begin{tabular}{l|l}
45 & CCGAACAACTCATCTTCTTC \\
\end{tabular} & 162 & CCT & ССТССТССТССТССТССТ & 18 \\
\hline Locus_615_Transcript_28/58_Confidence_0.419_Length_1952 & REVERSE & 55.93 & \begin{tabular}{l|l} 
42.86 TGCCCAAGTCTGATGTTACTA \\
\end{tabular} & & & & \\
\hline Locus_632_Transcript_4/8_Confidence_0.679_Length_1474 & FORWARD & 54.1 & \begin{tabular}{l|l|}
42.86 & GTTCTAAGCAAACTCCACATC \\
\end{tabular} & 167 & $\mathrm{CCT}$ & ССТССТССТССТССТССТССТ & 21 \\
\hline Locus_632_Transcript_4/8_Confidence_0.679_Length_1474 & REVERSE & 54.77 & 47.62 GAATAGGAGGAAGATGAGAGC & & & & \\
\hline Locus_699_Transcript_37/53_Confidence_0.525_Length_2441 & FORWARD & 55.27 & 38.1 GAGGATTCTTTCCATGAAATC & 171 & TTC & TTCTTCTTCTTCTTCTTC & 18 \\
\hline Locus_699_Transcript_37/53_Confidence_0.525_Length_2441 & REVERSE & 55.36 & 33.33 GTGTTCACCAAAGAAACAAAA & & & & \\
\hline Locus_774_Transcript_17/24_Confidence_0.648_Length_4742 & FORWARD & 54.84 & \begin{tabular}{l|l}
38.1 & AAGCTGTATGGTTTCCTTCTT \\
\end{tabular} & 143 & AGA & AGAAGAAGAAGAAGAAGAAGAAGAAGA & 27 \\
\hline Locus_774_Transcript_17/24_Confidence_0.648_Length_4742 & REVERSE & 55.01 & 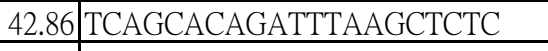 & & & & \\
\hline Locus_793_Transcript_11/15_Confidence_0.589_Length_956 & FORWARD & 54.93 & \begin{tabular}{l|l} 
42.86 & AATACACAGGTCTGGTTAGCA \\
\end{tabular} & 169 & $\mathrm{CAG}$ & CAGCAGCAGCAGCAGCAG & 18 \\
\hline Locus_793_Transcript_11/15_Confidence_0.589_Length_956 & REVERSE & 54.9 & \begin{tabular}{l|l}
42.86 & TTGGCTCTCTAACAACATAGC \\
\end{tabular} & & & & \\
\hline Locus_861_Transcript_27/41_Confidence_0.301_Length_1385 & FORWARD & 54.86 & 38.1 TTAATCCAATGTCTGAGGAGA & 150 & AGG & AGGAGGAGGAGGAGGAGG & 18 \\
\hline Locus_861_Transcript_27/41_Confidence_0.301_Length_1385 & REVERSE & 55.95 & 38.1 CGGCGGATTTATAATATGTCT & & & & \\
\hline Locus_925_Transcript_4/14_Confidence_0.620_Length_3080 & FORWARD & 54.91 & \begin{tabular}{l|l}
38.1 & AATCGACTGACTTCATCTTCA \\
\end{tabular} & 146 & $\mathrm{TCA}$ & TCATCATCATCATCATCATCA & 21 \\
\hline Locus_925_Transcript_4/14_Confidence_0.620_Length_3080 & REVERSE & 54.19 & 42.86 CTACCGAGTAATGACATGGAT & & & & \\
\hline Locus_925_Transcript_4/14_Confidence_0.620_Length_3080 & FORWARD & 54.7 & 33.33 CATCTCTTTTGTTTTGCAGAT & 157 & TCA & TCATCATCATCATCATCATCA & 21 \\
\hline Locus_925_Transcript_4/14_Confidence_0.620_Length_3080 & REVERSE & 55.33 & \begin{tabular}{|l|l|}
52.38 & GAGTCGGGGACACTTACTATC \\
\end{tabular} & & & & \\
\hline Locus_959_Transcript_15/21_Confidence_0.597_Length_1216 & FORWARD & 55.49 & 50 GGGGATACAGAAGATGGAGT & 142 & AGG & AGGAGGAGGAGGAGGAGG & 18 \\
\hline Locus_959_Transcript_15/21_Confidence_0.597_Length_1216 & REVERSE & 55.3 & \begin{tabular}{l|l|} 
42.86 & AGGATCGGTCGAATACACTAT \\
\end{tabular} & & & & \\
\hline Locus_1059_Transcript_25/31_Confidence_0.640_Length_1966 & FORWARD & 54.36 & 38.1 AGAAGTTCGATTCTGCTATGA & 153 & CGC & CGCCGCCGCCGCCGCCGCCGCCGC & 24 \\
\hline Locus_1059_Transcript_25/31_Confidence_0.640_Length_1966 & REVERSE & 55.12 & 42.86 GGGAAGGAAAGAGAGATGTAA & & & & \\
\hline Locus_1069_Transcript_41/64_Confidence_0.406_Length_2993 & FORWARD & 54.5 & \begin{tabular}{l|l}
47.62 & GAGGTGTTGAGTGTAGCTGTT \\
\end{tabular} & 156 & TCT & TCTTCTTCTTCTTCTTCT & 18 \\
\hline Locus_1069_Transcript_41/64_Confidence_0.406_Length_2993 & REVERSE & 55.33 & 38.1 ACCGGAATATAACAAACCCTA & & & & \\
\hline Locus_1090_Transcript_13/25_Confidence_0.630_Length_2059 & FORWARD & 55.34 & \begin{tabular}{l|l|} 
42.86 & ATCATACAAGGCAGACAACAG \\
\end{tabular} & 176 & $\mathrm{AGC}$ & AGCAGCAGCAGCAGCAGC & 18 \\
\hline
\end{tabular}


Locus_1090_Transcript_13/25_Confidence_0.630_Length_2059 Locus_1112_Transcript_10/44 Confidence 0.590 Length 3701 Locus_1112_Transcript_10/44_Confidence_0.590_Length_3701 Locus_1114_Transcript_6/30_Confidence_0.590_Length_2022 Locus_1114_Transcript_6/30_Confidence_0.590_Length_2022 Locus_1117_Transcript_10/11_Confidence_0.647_Length_907 Locus_1117_Transcript_10/11_Confidence_0.647_Length_907 Locus 1157 Transcript $7 / 12$ Confidence 0.685 Length 1449 Locus_1157_Transcript_7/12_Confidence_0.685_Length_1449 Locus_1158_Transcript_2/11_Confidence_0.688_Length_3223 Locus_1158_Transcript_2/11_Confidence_0.688_Length_3223 Locus_1158_Transcript_2/11_Confidence_0.688_Length_3223 Locus_1158_Transcript_2/11_Confidence_0.688_Length_3223 Locus_1161_Transcript_55/102_Confidence_0.536_Length_2614 Locus_1161_Transcript_55/102_Confidence_0.536_Length_2614 Locus 1195 Transcript 51/74 Confidence 0.470 Length 2688 Locus_1195_Transcript_51/74_Confidence_0.470_Length_2688 Locus_1195_Transcript 51/74 Confidence 0.470 Length 2688 Locus_1195_Transcript_51/74_Confidence_0.470_Length_2688 Locus_1220_Transcript_16/18_Confidence_0.543_Length_2694 Locus_1220_Transcript_16/18 Confidence 0.543 Length 2694 Locus_1276_Transcript_3/10_Confidence_0.638_Length_1556 Locus_1276_Transcript_3/10_Confidence_0.638_Length_1556 Locus 1338 Transcript 4/11 Confidence 0.671 Length 3687 Locus_1338_Transcript_4/11_Confidence_0.671_Length_3687 Locus_1358_Transcript_12/26_Confidence_0.596_Length_1705 Locus 1358 Transcript 12/26 Confidence 0.596 Length 1705 Locus_1382_Transcript_31/44_Confidence_0.498_Length_2303 Locus_1382_Transcript_31/44_Confidence_0.498_Length_2303 Locus 1385 Transcript 32/36 Confidence 0.455 Length 1324 Locus_1385_Transcript_32/36_Confidence_0.455_Length_1324 Locus 1390 Transcript 27/33 Confidence 0.586 Length 1949 Locus_1390_Transcript_27/33_Confidence_0.586_Length_1949 Locus_1433_Transcript_149/150_Confidence_0.287_Length_3713 Locus 1433 Transcript 149/150 Confidence 0.287 Length_3713 Locus_1435_Transcript_6/11_Confidence_0.708_Length_2457 Locus_1435_Transcript 6/11 Confidence 0.708 Length 2457 Locus 1437 Transcript 8/18 Confidence_0.614 Length 2264 Locus_1437_Transcript_8/18_Confidence_0.614_Length_2264 Locus_1461_Transcript_31/88_Confidence_0.333_Length_3170 Locus_1461_Transcript_31/88_Confidence_0.333_Length_3170

\begin{tabular}{|c|c|c|c|c|c|c|}
\hline REVERSE & 55.62 & \begin{tabular}{l|l}
47.62 & GAGTTGGCTCTTAGACATTCC \\
\end{tabular} & & & & \\
\hline FORWARD & 55.18 & $\begin{array}{l}42.86 \\
\text { GGGATTTAGGTATATGGATCG } \\
\end{array}$ & 172 & GGT & GGTGGTGGTGGTGGTGGT & 18 \\
\hline REVERSE & 54.94 & \begin{tabular}{l|l|l|} 
50 & CATGCTCAACCTTCTCCTAC \\
\end{tabular} & & & & \\
\hline FORWARD & 53.11 & \begin{tabular}{|l|l|}
34.78 & ACATATCATACTTACCCACTCA \\
\end{tabular} & 146 & AAT & AATAATAATAATAATAAT & 18 \\
\hline REVERSE & 54.04 & \begin{tabular}{l|l|l}
30.43 & AGTTGGTGTTATAATTGTTGTCA \\
\end{tabular} & & & & \\
\hline FORWARD & 55.22 & 38.1 ATCATAGAAAGATGAGCAGCA & 256 & $\mathrm{GCC}$ & GCCGCCGCCGCCGCCGCCGCC & 21 \\
\hline REVERSE & 54.9 & \begin{tabular}{l|l|l|} 
42.86 & AGAGGCTGTTAAGTTCACT \\
\end{tabular} & & & & \\
\hline FORWARD & 54.04 & 27.27 AAATTGGACCCTATAAGAAAAA & 147 & TTG & TTGTTGTTGTTGTTGTTGTTGTTGTTGTTGT & 33 \\
\hline REVERSE & 54.57 & 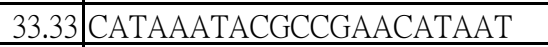 & & & & \\
\hline FORWARD & 54.22 & 38.1 GAAAGAAGAAAAGGAAGAAGG & 154 & AGG & AGGAGGAGGAGGAGGAGGAGGAGGAGG & 27 \\
\hline REVERSE & 54.79 & \begin{tabular}{l|l|l}
47.62 & TACTACCCTCGTCAGCTATTG \\
\end{tabular} & & & & \\
\hline FORWARD & 54.79 & \begin{tabular}{|l|l|} 
42.86 & CAGAGCAGAAGAAGAATAAG \\
\end{tabular} & 151 & $\mathrm{AGG}$ & AGGAGGAGGAGGAGGAGGAGGAGGAGG & 27 \\
\hline REVERSE & 55.05 & 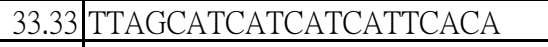 & & & & \\
\hline FORWARD & 55.63 & \begin{tabular}{l|l|} 
42.86 & ACCGAGGATCTGAGAGTTTTA \\
\end{tabular} & 145 & CTT & CTTCTTCTTCTTCTTCTT & 18 \\
\hline REVERSE & 55.07 & \begin{tabular}{l|l|l}
33.33 & AAACAGTCCAATTAAACAGCA \\
\end{tabular} & & & & \\
\hline FORWARD & 54.74 & \begin{tabular}{l|l|l|}
33.33 & GATTGATTCTTTGCTTGAGAA \\
\end{tabular} & 151 & CTT & CTTCTTCTTCTTCTTCTT & 18 \\
\hline REVERSE & 55 & \begin{tabular}{l|l|}
38.1 & AGATGCTGATGAAGATGAAGA \\
\end{tabular} & & & & \\
\hline FORWARD & 54.97 & \begin{tabular}{l|l|}
47.62 & CCTCATCTCCCTCTAACTCAT \\
\end{tabular} & 144 & CTT & CTTCTTCTTCTTCTTCTT & 18 \\
\hline REVERSE & 55.06 & 47.62 GAGGAAGAGAAGAAGAGAACG & & & & \\
\hline FORWARD & 57.51 & \begin{tabular}{|l|l|}
50 & CTATGAGCCCGGTGATGTAT \\
\end{tabular} & 156 & $\mathrm{AAG}$ & $\mathrm{SAAG}$ & 18 \\
\hline REVERSE & 54.76 & $\begin{array}{l}42.86 \\
\text { TTTGCTCTCTGACCTTAACAC } \\
\end{array}$ & & & & \\
\hline FORWARD & 54.71 & 42.86 6 СTTTTCACTTCTCTCCACTCA & 153 & ATC & ATCATCATCATCATCATCATC & 21 \\
\hline REVERSE & 55.34 & \begin{tabular}{|l|l|} 
42.86 & GAGGACTTGTTGTTTCTGACA \\
\end{tabular} & & & & \\
\hline FORWARD & 55.12 & \begin{tabular}{l|l|l}
33.33 & AAATTATGAAGGGTGGAACAT \\
\end{tabular} & 141 & TTA & TTATTATTATTATTATTATTA & 21 \\
\hline REVERSE & 56.08 & $\begin{array}{ll}35 & \text { TTTTTAAAGCAGCAAACAGC } \\
\end{array}$ & & & & \\
\hline FORWARD & 54.66 & 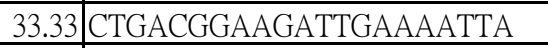 & 165 & TCA & TCATCATCATCATCATCATCATCA & 24 \\
\hline REVERSE & 54.59 & 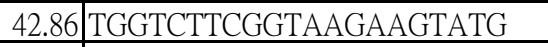 & & & & \\
\hline FORWARD & 54.88 & $\begin{array}{l}\text { 42.86 } \\
\text { CAGATGCTGATAAAGATGGAC } \\
\end{array}$ & 151 & TGA & TGATGATGATGATGATGA & 18 \\
\hline REVERSE & 54.82 & \begin{tabular}{l|l|}
38.1 & AAACTTCTAACCTGGAAAGGA \\
\end{tabular} & & & & \\
\hline FORWARD & 54.77 & \begin{tabular}{l|l|}
33.33 & TGAATCAGTGTTTTCTGGAAT \\
\end{tabular} & 143 & GAA & GAAGAAGAAGAAGAAGAA & 18 \\
\hline REVERSE & 55.01 & \begin{tabular}{l|l|}
38.1 & CAAATACAATTCCСТСТССТ \\
\end{tabular} & & & & \\
\hline FORWARD & 54.98 & 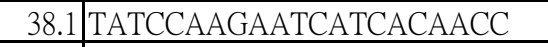 & 169 & TTA & TTATTATTATTATTATTATTA & 21 \\
\hline REVERSE & 55.14 & $\begin{array}{l}42.86 \text { GAGGAGAGAATGCCTGTTTAT } \\
\end{array}$ & & & & \\
\hline FORWARD & 54.82 & \begin{tabular}{|l|l|}
38.1 & GCGACTATAATCTGCCAAATA \\
\end{tabular} & 156 & GAA & GAAGAAGAAGAAGAAGAA & 18 \\
\hline REVERSE & 55.24 & 52.38 CTCTCTCTGTCTCCCTCTGTT & & & & \\
\hline FORWARD & 55.38 & \begin{tabular}{l|l|l} 
42.86CTGGAGAAGGTTAATCAG \\
\end{tabular} & 151 & CAT & CATCATCATCATCATCAT & 18 \\
\hline REVERSE & 54.75 & 38.1 1 TATTGTACAACCACCACATGA & & & & \\
\hline FORWARD & 54.65 & \begin{tabular}{|l|l|}
38.1 & TCAGTTTTCCTCATTCTCTG \\
\end{tabular} & 148 & AGC & AGCAGCAGCAGCAGCAGC & 18 \\
\hline REVERSE & 54.4 & \begin{tabular}{l|l|l}
47.62 & GAAGTAGTGCAGATCTCAGC \\
\end{tabular} & & & & \\
\hline FORWARD & 54.44 & \begin{tabular}{l|l}
38.1 & CGTAATTTAGGGTTTCCTTCT \\
\end{tabular} & 149 & TCT & TCTTCTTCTTCTTCTTCT & 18 \\
\hline REVERSE & 55.16 & $\begin{array}{l}28.57 \text { ATCAAACGCATCAAAACTAAA } \\
\end{array}$ & & & & \\
\hline
\end{tabular}


Locus_1461_Transcript_31/88_Confidence_0.333_Length_3170 Locus_1461_Transcript_31/88_Confidence_0.333_Length_3170 Locus_1514_Transcript_7/39_Confidence_0.529_Length_2012 Locus_1514_Transcript_7/39_Confidence_0.529_Length_2012 Locus_1520_Transcript_36/42_Confidence_0.490_Length_1086 Locus_1520_Transcript_36/42_Confidence_0.490_Length_1086 Locus_1569_Transcript_22/26_Confidence_0.686_Length_3296 Locus 1569 Transcript 22/26 Confidence 0.686 Length 3296 Locus_1636_Transcript_37/46_Confidence_0.591_Length_2024 Locus_1636_Transcript_37/46_Confidence_0.591_Length_2024 Locus_1705_Transcript_37/42_Confidence_0.546_Length_1219 Locus_1705_Transcript_37/42_Confidence_0.546_Length_1219 Locus_1733_Transcript_6/15_Confidence_0.594_Length_1614 Locus_1733_Transcript_6/15_Confidence_0.594_Length_1614 Locus_1737_Transcript_8/27_Confidence_0.494_Length_2012 Locus_1737_Transcript_8/27_Confidence_0.494_Length_2012 Locus_1756_Transcript_103/107_Confidence_0.282_Length_2877 Locus_1756_Transcript_103/107_Confidence_0.282_Length_2877 Locus_1756_Transcript_103/107 Confidence 0.282 Length 2877 Locus_1756_Transcript_103/107_Confidence_0.282_Length_2877 Locus 1792 Transcript 40/42 Confidence 0.485 Length 1340 Locus_1792_Transcript_40/42_Confidence_0.485_Length_1340 Locus 1792 Transcript 40/42 Confidence 0.485 Length 1340 Locus 1792 Transcript 40/42 Confidence 0.485 Length 1340 Locus_1831_Transcript_13/38_Confidence_0.546_Length_2408 Locus_1831_Transcript_13/38_Confidence_0.546_Length_2408 Locus 1904 Transcript 18/32 Confidence 0.594 Length 9055 Locus_1904_Transcript_18/32_Confidence_0.594_Length_9055 Locus 1914 Transcript 14/23 Confidence 0.495 Length 3518 Locus 1914 Transcript 14/23 Confidence 0.495 Length 3518 Locus_1915_Transcript_12/38_Confidence_0.608_Length_1915 Locus 1915 Transcript 12/38 Confidence 0.608 Length 1915 Locus_1926_Transcript_14/28_Confidence_0.611_Length_1072 Locus 1926 Transcript 14/28 Confidence 0.611 Length 1072 Locus 2029 Transcript 32/44 Confidence 0.599 Length 2413 Locus_2029_Transcript_32/44_Confidence_0.599_Length_2413 Locus_2075_Transcript 7/14 Confidence 0.652 Length 1348 Locus 2075 Transcript 7/14 Confidence 0.652 Length 1348 Locus_2075_Transcript_7/14_Confidence_0.652_Length_1348 Locus_2075_Transcript_7/14_Confidence_0.652_Length_1348 Locus_2078_Transcript_22/29_Confidence_0.598_Length_3107

\begin{tabular}{|c|c|c|c|c|c|c|}
\hline FORWARD & 55.05 & \begin{tabular}{l|l}
38.1 & TGGGTAATTATGGTTCTGATG \\
\end{tabular} & 153 & GAT & GATGATGATGATGATGATGAT & 21 \\
\hline REVERSE & 54.34 & $\begin{array}{l}\text { 42.86 } \\
\text { TCCTTTACTGTCTCAACATCC } \\
\end{array}$ & & & & \\
\hline FORWARD & 54.61 & \begin{tabular}{l|l|l|}
33.33 & AGGCTTTCATATACCCAAAAT \\
\end{tabular} & 150 & TAT & TATTATTATTATTATTATTATTAT & 24 \\
\hline REVERSE & 54.95 & \begin{tabular}{l|l|l|}
31.82 & TGTGACTTGAAATGTTGTTTCT \\
\end{tabular} & & & & \\
\hline FORWARD & 55.54 & \begin{tabular}{l|l}
38.1 & ATCAGCCTTCATGATCTTCTT \\
\end{tabular} & 152 & GCT & GCTGCTGCTGCTGCTGCTGCTGCT & 24 \\
\hline REVERSE & 55.31 & \begin{tabular}{|l|l|}
50 & AACTCTACCACCATCAGCAG \\
\end{tabular} & & & & \\
\hline FORWARD & 54.77 & 45.45 GACGTTATAAAGGGTACTGAGG & 179 & TTC & TTCTTCTTCTTCTTCTTCTTCTTC & 24 \\
\hline REVERSE & 54.71 & 47.62 СCTTCTTCTTCCTCTATGTCC & & & & \\
\hline FORWARD & 57.57 & $\begin{array}{l}\text { 42.86 ATGTCAAGGTGAAGGAGGAAT } \\
\end{array}$ & 209 & GAG & GAGGAGGAGGAGGAGGAG & 18 \\
\hline REVERSE & 55.21 & $\begin{array}{l}40.91 \\
\text { GTATCCAATCATCCGAACTATC } \\
\end{array}$ & & & & \\
\hline FORWARD & 54.62 & $\begin{array}{l}45 \\
\text { CGATTCCATAAGATCTCCAC } \\
\end{array}$ & 162 & CCT & ССТССТССТССТССТССТССТ & 21 \\
\hline REVERSE & 55.03 & \begin{tabular}{l|l} 
47.62 & CCACCACCTCCTAGTTTTACT \\
\end{tabular} & & & & \\
\hline FORWARD & 54.88 & $\begin{array}{l}38.1 \\
\end{array}$ & 145 & CCT & ССТССТССТССТСТССТ & 18 \\
\hline REVERSE & 55.2 & 42.86 GAGAGGAAGAATCGGATAAGA & & & & \\
\hline FORWARD & 55.39 & 42.86 GGATTGGTCATGGGTATAGTT & 130 & GAT & GATGATGATGATGATGAT & 18 \\
\hline REVERSE & 54.91 & 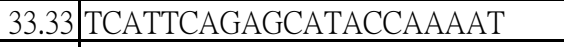 & & & & \\
\hline FORWARD & 54.58 & \begin{tabular}{l|l}
38.1 & CCAATAAAGCTTCTGTGTCAT \\
\end{tabular} & 139 & TCA & TCATCATCATCATCATCATCA & 21 \\
\hline REVERSE & 54.46 & 38.1 CTTGAAGGAGGAAAAAGAGAT & & & & \\
\hline FORWARD & 55.14 & \begin{tabular}{l|l}
40 & GCGTTTCCTTAGAATTTGTG \\
\end{tabular} & 160 & TCA & TCATCATCATCATCATCATCATCA & 24 \\
\hline REVERSE & 54.97 & 33.33 TTGGACCTGAAATCTTAAACA & & & & \\
\hline FORWARD & 54.37 & \begin{tabular}{l|l|}
42.86 & CGTCATCTTTGGTCTGTCTAT \\
\end{tabular} & 140 & TCT & TCTTCTTCTTCTTCTTCTTCTTCT & 24 \\
\hline REVERSE & 54.94 & \begin{tabular}{l|l} 
36.36 AGCAAACAAGAAGAAGAAGAAG \\
\end{tabular} & & & & \\
\hline FORWARD & 54.98 & \begin{tabular}{|l|l|} 
42.86 & CTCGCTCTTCTTCTTCTTCTT \\
\end{tabular} & 149 & TTC & TTCTTCTTCTTCTTCTTCTTCTTC & 24 \\
\hline REVERSE & 55.3 & $\begin{array}{l}\text { 42.86 } \\
\text { ATCTGTACGGTAACACAAACG } \\
\end{array}$ & & & & \\
\hline FORWARD & 55.3 & \begin{tabular}{l|l|l|}
47.62 & GTCTCCATTTAAGTGGTCTCC \\
\end{tabular} & 135 & TCT & TCTTCTTCTTCTTCTTCTTCTTCT & 24 \\
\hline REVERSE & 55.27 & $\begin{array}{l}33.33 \text { CGAACAACGTAATTGATTGAT } \\
\end{array}$ & & & & \\
\hline FORWARD & 55.4 & $\begin{array}{ll}33.33 & \text { GGTTGCATTTGAACTTGAATA } \\
\end{array}$ & 180 & GCG & GCGGCGGCGGCGGCGGCGGCGGCG & 24 \\
\hline REVERSE & 54.95 & \begin{tabular}{|l|l|}
33.33 & CCCCAATTCTCAAATTTCTAT \\
\end{tabular} & & & & \\
\hline FORWARD & 55.14 & 50 GCAGAAACAGAGGAAGACAG & 147 & GAT & GATGATGATGATGATGATGATGATGATGA & 33 \\
\hline REVERSE & 54.19 & $\begin{array}{l}38.1 \\
\end{array}$ & & & & \\
\hline FORWARD & 54.75 & \begin{tabular}{ll|l}
33.33 & ATGAGTTTGTTTTGGTTGGTA \\
\end{tabular} & 153 & ATG & ATGATGATGATGATGATG & 18 \\
\hline REVERSE & 55.09 & 38.1 GTATGTAAATGCCCCATAACA & & & & \\
\hline FORWARD & 55.1 & 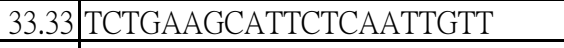 & 150 & TCT & TCTTCTTCTTCTTCTTCT & 18 \\
\hline REVERSE & 55.15 & 42.86 AGAGAGAGCAACATGAACAGA & & & & \\
\hline FORWARD & 54.49 & \begin{tabular}{|l|l|}
38.1 & GCTAACCAGTTCACAAACAAT \\
\end{tabular} & 155 & CGG & CGGCGGCGGCGGCGGCGG & 18 \\
\hline REVERSE & 55.2 & $\begin{array}{l}38.1 \\
\end{array}$ & & & & \\
\hline FORWARD & 55.75 & \begin{tabular}{l|l}
38.1 & GGATGAATCAGCTATGGATTT \\
\end{tabular} & 151 & TTC & TTCTTCTTCTTCTTCTTCTTC & 21 \\
\hline REVERSE & 54.94 & 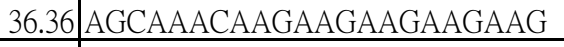 & & & & \\
\hline FORWARD & 54.56 & $\begin{array}{l}42.86 \\
\text { TGTGCTTCTAGAGAGGAAGAA } \\
\end{array}$ & 167 & TTC & TTCTTCTTCTTCTTCTTCTTCTTC & 24 \\
\hline REVERSE & 55.67 & 40.91 GGAGCAACTATACATTTCCTTG & & & & \\
\hline FORWARD & 55.72 & 52.38 CACCTATCACACGACCAGTAG & 150 & GGT & GGTGGTGGTGGTGGTGGT & 18 \\
\hline
\end{tabular}


Locus_2078_Transcript_22/29_Confidence_0.598_Length_3107 Locus_2093_Transcript_28/37_Confidence 0.576 Length 994 Locus_2093_Transcript_28/37_Confidence_0.576_Length_994 Locus 2319 Transcript 15/30 Confidence 0.625 Length 3196 Locus 2319 Transcript 15/30 Confidence 0.625 Length 3196 Locus_2350_Transcript_8/10_Confidence_0.683_Length_2253 Locus_2350_Transcript_8/10_Confidence_0.683_Length_2253 Locus 2350 Transcript $8 / 10$ Confidence 0.683 Length 2253 Locus_2350_Transcript_8/10_Confidence_0.683_Length_2253 Locus 2400 Transcript 10/26 Confidence 0.607 Length 2492 Locus_2400_Transcript_10/26_Confidence_0.607_Length_2492 Locus_2434_Transcript_15/28_Confidence_0.545_Length_2352 Locus 2434 Transcript 15/28 Confidence 0.545 Length 2352 Locus_2444_Transcript_13/49_Confidence_0.611_Length_2252 Locus_2444_Transcript_13/49_Confidence_0.611_Length_2252 Locus_2496_Transcript_18/28_Confidence_0.538_Length_1997 Locus_2496_Transcript_18/28_Confidence_0.538_Length_1997 Locus 2592 Transcript 68/83 Confidence 0.594 Length 4085 Locus 2592 Transcript 68/83 Confidence_0.594_Length_4085 Locus_2610_Transcript_27/50_Confidence_0.610_Length_15561 Locus 2610 Transcript_27/50 Confidence 0.610 Length 1556 Locus_2726_Transcript_5/13_Confidence_0.618_Length_2238 Locus 2726 Transcript 5/13 Confidence 0.618 Length 2238 Locus 2726 Transcript 5/13 Confidence 0.618 Length 2238 Locus_2726_Transcript_5/13_Confidence_0.618_Length_2238 Locus_2774_Transcript_13/23_Confidence_0.437_Length_2369 Locus 2774 Transcript 13/23 Confidence 0.437 Length 2369 Locus_2776_Transcript_4/11_Confidence_0.677_Length_1835 Locus 2776 Transcript 4/11 Confidence 0.677 Length 1835 Locus_2776_Transcript_4/11_Confidence_0.677_Length_1835 Locus_2776_Transcript_4/11_Confidence_0.677_Length_1835 Locus_2818_Transcript_29/73_Confidence_0.469_Length_3397 Locus_2818_Transcript_29/73_Confidence_0.469_Length_3397 Locus_2857_Transcript_15/16_Confidence_0.649_Length_6622 Locus 2857 Transcript 15/16 Confidence 0.649 Length 6622 Locus_2865_Transcript_1/6_Confidence_0.636_Length_833 Locus_2865_Transcript_1/6_Confidence_0.636_Length_833

Locus 2923_Transcript 33/44 Confidence 0.436 Length 1186 Locus_2923_Transcript_33/44_Confidence_0.436_Length_1186 Locus_2960_Transcript_12/17_Confidence_0.578_Length_6370 Locus_2960_Transcript_12/17_Confidence_0.578_Length_6370

\begin{tabular}{|c|c|c|c|c|c|c|}
\hline REVERSE & 55.43 & \begin{tabular}{l|l|} 
42.86 & CAAGTCTTCAAGCTCAAACAG \\
\end{tabular} & & & & \\
\hline FORWARD & 55.58 & \begin{tabular}{l|l|} 
42.86 & СTCTTCTTCTGTTTGGTTCT \\
\end{tabular} & 149 & CTG & CTGCTGCTGCTGCTGCTGCTGCTGCTG & 27 \\
\hline REVERSE & 55.47 & \begin{tabular}{l|l|l}
47.62 & TCAGCATAGTATCCACTACGG \\
\end{tabular} & & & & \\
\hline FORWARD & 55.04 & 38.1 AAAACACAAACATGGTCTCAC & 160 & ATT & ATTATTATTATTATTATT & 18 \\
\hline REVERSE & 55.63 & \begin{tabular}{l|l|l|}
45 & CCTTGTTGCCTGAACTTCTA \\
\end{tabular} & & & & \\
\hline FORWARD & 54.2 & \begin{tabular}{|l|l|} 
42.86ATTAGTTGTACTCCAAAGC \\
\end{tabular} & 151 & GAT & GATGATGATGATGATGAT & 18 \\
\hline REVERSE & 55.57 & \begin{tabular}{l|l|}
33.33 & TCATCATCATCATCTTCGTT \\
\end{tabular} & & & & \\
\hline FORWARD & 54.92 & 38.1 CTCCATTAAACGAAGATGATG & 149 & GAT & GATGATGATGATGATGATGAT & 21 \\
\hline REVERSE & 55.04 & \begin{tabular}{|l|l|}
38.1 & ATGTTGTGTGTTGGTCTC \\
\end{tabular} & & & & \\
\hline FORWARD & 54.89 & 42.86 TCCTCCTCACTTCTTCTTCTT & 117 & TTC & TTCTTCTTCTTCTTCTTCTTC & 21 \\
\hline REVERSE & 54.77 & 38.1 TCGCAGTTGTATTTTAGTTCC & & & & \\
\hline FORWARD & 54.99 & \begin{tabular}{l|l|} 
42.8AGTCCTATCATCTTCCCAAG \\
\end{tabular} & 143 & TTG & TTGTTGTTGTTGTTGTTGTTG & 21 \\
\hline REVERSE & 55.25 & \begin{tabular}{l|l|} 
42.86ACAGACTCACGCATACATA \\
\end{tabular} & & & & \\
\hline FORWARD & 56.33 & \begin{tabular}{l|l|}
50 & CTTCCCTTCACCTCCATAAG \\
\end{tabular} & 148 & TCC & ТССТССТССТССТССТСС & 18 \\
\hline REVERSE & 54.24 & \begin{tabular}{l|l|l}
42.86 & TGTGAGCTTGGTAGAGAGATT \\
\end{tabular} & & & & \\
\hline FORWARD & 54.82 & $\begin{array}{l}42.86 \text { CATATGGTTGACAAGGAAGAG } \\
\end{array}$ & 209 & GAA & GAAGAAGAAGAAGAAGAA & 18 \\
\hline REVERSE & 54.23 & \begin{tabular}{|l|l|}
42.86 & GATCATTACCTCATCGGAGTA \\
\end{tabular} & & & & \\
\hline FORWARD & 54.73 & 30 TTTTTGGGTTAAAAATACGC & 149 & TAA & TAATAATAATAATAATAATAA & 21 \\
\hline REVERSE & 54.73 & \begin{tabular}{l|l}
38.1 & TCTCTGAATTCTTCTGATTCG \\
\end{tabular} & & & & \\
\hline FORWARD & 55.36 & \begin{tabular}{l|l|l}
42.86 & TGGTACTTGACGAAATCAG \\
\end{tabular} & 158 & TGA & TGATGATGATGATGATGA & 18 \\
\hline REVERSE & 54.11 & \begin{tabular}{l|l|l}
47.62 & AGAGGCTAGTTCCTTCTCACT \\
\end{tabular} & & & & \\
\hline FORWARD & 55.22 & 38.1 CTCGATCATCATGAAAACTGT & 144 & CAT & CATCATCATCATCATCAT & 18 \\
\hline REVERSE & 55.22 & \begin{tabular}{|l|l|}
42.86 & GGCTTACCTCAGAAACTGAT \\
\end{tabular} & & & & \\
\hline FORWARD & 55.22 & \begin{tabular}{l|l|}
38.1 & CTCGATCATCATGAAAACTGT \\
\end{tabular} & 144 & TCT & TCTTCTTCTTCTTCTTCTTCT & 21 \\
\hline REVERSE & 55.22 & \begin{tabular}{|l|l|}
42.86 & GGCTTACCTCAGAAACTGAT \\
\end{tabular} & & & & \\
\hline FORWARD & 55.31 & 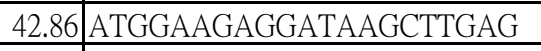 & 157 & AGA & AGAAGAAGAAGAAGAAGA & 18 \\
\hline REVERSE & 54.96 & 33.33 GGTTTTTCTTTTTCTTCTTGG & & & & \\
\hline FORWARD & 54.71 & \begin{tabular}{l|l|l}
47.62 & GAAGAGTATTTGAGGAGGAG \\
\end{tabular} & 147 & GGA & GGAGGAGGAGGAGGAGGAGGA & 21 \\
\hline REVERSE & 55.76 & \begin{tabular}{l|l|l}
42.86 & CCTTGGAGAAGAGATGAAG \\
\end{tabular} & & & & \\
\hline FORWARD & 54.76 & \begin{tabular}{l|l|l}
33.33 & ATTAATGGCGGAAGAGTATTT \\
\end{tabular} & 157 & GGA & GGAGGAGGAGGAGGAGGAGGA & 21 \\
\hline REVERSE & 55.76 & \begin{tabular}{l|l|l}
42.86 & CCTTGGAGAAGAGATGAAG \\
\end{tabular} & & & & \\
\hline FORWARD & 55.14 & \begin{tabular}{l|l|l|}
33.33 & CCACATAACCAAACAATTA \\
\end{tabular} & 150 & AAT & AATAATAATAATAATAAT & 18 \\
\hline REVERSE & 55.09 & \begin{tabular}{l|l|}
42.86 & GTTTGAGGTTTCTGAAGGACT \\
\end{tabular} & & & & \\
\hline FORWARD & 55.09 & \begin{tabular}{l|l|}
38.1 & GACATGGATAGTGTTGCAAAT \\
\end{tabular} & 170 & ATG & ATGATGATGATGATGATGATG & 21 \\
\hline REVERSE & 55.36 & 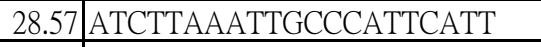 & & & & \\
\hline FORWARD & 54.39 & 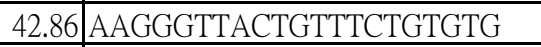 & 103 & GGT & GGTGGTGGTGGTGGTGGT & 18 \\
\hline REVERSE & 55.19 & 38.1 TGCAAATATCTACACCAATCC & & & & \\
\hline FORWARD & 54.81 & \begin{tabular}{l|l|}
47.62 & GATGAGAGGAAGAAGAGGAG \\
\end{tabular} & 152 & GAT & GATGATGATGATGATGATGAT & 21 \\
\hline REVERSE & 55.57 & \begin{tabular}{l|l|}
50 & AGGTACTCTGTCCCCATTTC \\
\end{tabular} & & & & \\
\hline FORWARD & 54.58 & 42.86 CTATGCCTTCTCATCCTGATA & 151 & $\mathrm{CAG}$ & CAGCAGCAGCAGCAGCAG & 18 \\
\hline REVERSE & 54.49 & $\begin{array}{l}28.57 \mid \text { ATGTTTGTTGGTGATTGATTT } \\
\end{array}$ & & & & \\
\hline
\end{tabular}


Locus_2971_Transcript_15/22_Confidence_0.580_Length_1720 Locus_2971_Transcript_15/22_Confidence_0.580_Length 1720 Locus_3006_Transcript_114/121_Confidence_0.164_Length_3674 Locus_3006_Transcript_114/121_Confidence_0.164_Length_3674 Locus 3024 Transcript_6/10 Confidence 0.664 Length 3980 Locus_3024_Transcript_6/10_Confidence_0.664_Length_3980 Locus 3116_Transcript 11/19_Confidence_0.570_Length_2489 Locus 3116 Transcript 11/19 Confidence 0.570 Length 2489 Locus_3222_Transcript_15/39_Confidence_0.689_Length_2347 Locus 3222 Transcript 15/39 Confidence 0.689 Length 2347 Locus_3243_Transcript_22/41_Confidence_0.493_Length_3919 Locus_3243_Transcript_22/41_Confidence_0.493_Length_3919 Locus_3268_Transcript_16/27_Confidence_0.381_Length_1825 Locus_3268_Transcript_16/27_Confidence_0.381_Length_1825 Locus_3298_Transcript_21/30_Confidence_0.628_Length_1363 Locus_3298_Transcript 21/30_Confidence_0.628_Length_1363 Locus_3327_Transcript_38/48_Confidence_0.421_Length_1690 Locus_3327_Transcript_38/48_Confidence_0.421_Length_1690 Locus_3355_Transcript_3/18_Confidence_0.579_Length_2015 Locus_3355_Transcript_3/18_Confidence_0.579_Length_2015 Locus_3376_Transcript_101/115_Confidence_0.336_Length_5494 Locus_3376_Transcript_101/115_Confidence_0.336_Length_5494 Locus_3421_Transcript_8/29_Confidence_0.429_Length_1397 Locus 3421 Transcript 8/29 Confidence 0.429 Length 1397 Locus_3428_Transcript_25/50_Confidence_0.458_Length_1306 Locus_3428_Transcript_25/50_Confidence_0.458_Length_1306 Locus 3428 Transcript 25/50 Confidence 0.458 Length 1306 Locus_3428_Transcript_25/50_Confidence_0.458_Length_1306 Locus_3428_Transcript_25/50_Confidence_0.458_Length_1306 Locus_3428_Transcript_25/50_Confidence_0.458_Length_1306 Locus_3489_Transcript_1/8_Confidence_0.632_Length_2642 Locus_3489_Transcript_1/8_Confidence_0.632_Length_2642

Locus_3510_Transcript_22/30_Confidence_0.617_Length_1724 Locus 3510 Transcript 22/30 Confidence 0.617 Length 1724 Locus 3575 Transcript 34/54 Confidence 0.581 Length 1836 Locus_3575_Transcript_34/54_Confidence_0.581_Length_1836 Locus_3600_Transcript_10/15_Confidence_0.646_Length_1477 Locus_3600_Transcript_10/15_Confidence_0.646_Length_1477 Locus_3601_Transcript_12/13_Confidence_0.607_Length_2230 Locus_3601_Transcript_12/13_Confidence_0.607_Length_2230 Locus_3601_Transcript_12/13_Confidence_0.607_Length_2230
FORWARD REVERSE FORWARD REVERSE FORWARD REVERSE FORWARD REVERSE FORWARD REVERSE FORWARD REVERSE FORWARD REVERSE FORWARD REVERSE FORWARD REVERSE FORWARD REVERSE FORWARD REVERSE FORWARD REVERSE FORWARD REVERSE FORWAR REVERSE FORWARD REVERSE FORWARD REVERSE FORWARD REVERSE FORWARD REVERSE FORWARD REVERSE FORWARD REVERSE FORWARD \begin{tabular}{l|l|l|l|l}
38.1 & TTCTGAGGAAAGAGGAGAAAT & 150 & GCG & GCGGCGGCGGCGGCGGCGGCG
\end{tabular} 33.33 TTGTTCTCTATTTCGAAGCAT 33.33 CAATTCTGGATTTTGATTCTG 28.57 TAATTTGAATCACCAATGCTT 40 TAACGAACGATTGGCTCTAT 50 GTTCCTCGACGAGAGTTTAC 42.86 TTCCTCATCTCTACAACCTCA 33.33 TAGATCGTGTTTTTGTTTTGG 38.1 GAGTATTGAATCCCCAAGTTT 38.1 TTCAGAATCATCTTTCTCCTG 38.1 TCTTACGGGATTTCACAGTTA 38.1 TCTCATGGAATTCGCTACTTA 45 TAACTCGCCTTCTCGTCTTA

55 27.27 TTTTTCCATTACTGTTTGATGA \begin{tabular}{l|l}
55.22 & 47.62 CAGAGAAGGAGAAGAAGAAGC \\
\hline
\end{tabular} 42.86 TAACAACTTACACCTGCTCGT 42.86 AATCTAACTCAAAGCCCTCTC 42.86 CATATCCAGAACTCGTCATTC 47.62 CCTAATCCAGTGGTGTCTGTA

45 AGCAGCAGTCTCTGATGATT 52.38 GGCTAGGTAGAGAGAAACCTG 38.1 TGGCGAGACAATAAGTAAGAA 33.33 GTCATCCAAGTTGCATATTTT 42.86 AGTTTGGCATCTTCCTCTAGT

47.62 CCTCTTCTTCCCAGAAACTAC 33.33 GAAGCAAACAGAAGAAAAGAA 42.86 CACACAGCTGTTAATTTAGCC 38.1 TGGATTCCATAGAAGAGAGAA 33.33 ACCATCAATGTTGAAGAACAA 38.1 TGGATTCCATAGAAGAGAGAA 38.1 TCTTGAACATAAACAGGGAAG 40 ACCTTCACATCACCAAAAAG 33.33 TAATGCGAAACCCTATGTAAA 28.57 AAAATTTTCTTCTCGAATGTG 50 GTATCCCTGATCTGCTGCTT 38.1 ACCGTTGAGATTATTGGAAGT 42.86 ATCTCTGTCGCCTTCACTATT 38.1 TTTTGTTCTTCTGATGCTAGG 42.86 CTTCAGGTGTTGTGTGAGATA 42.86 CACCGAATACACTCTTCACAT 33.33 TCCTTGCTGATTTCTGTTATT

\begin{tabular}{|c|c|c|c|}
\hline & GCG & GCGGCGGCGGCGGCGGCGGCG & 21 \\
\hline 154 & TTA & TTATTATTATTATTATTATTA & 21 \\
\hline 133 & $\mathrm{GCC}$ & GCCGCCGCCGCCGCCGCCGCC & 21 \\
\hline 151 & CCG & CCGCCGCCGCCGCCGCCGCCG & 21 \\
\hline 150 & GAG & GAGGAGGAGGAGGAGGAGGAGGAG & 24 \\
\hline & & & \\
\hline 135 & $\mathrm{AAG}$ & AAGAAGAAGAAGAAGAAG & 18 \\
\hline 166 & $\mathrm{AAC}$ & AACAACAACAACAACAACAACAACAAC & 27 \\
\hline & & & \\
\hline 169 & AGA & AGAAGAAGAAGAAGAAGAAGAAGA & 24 \\
\hline 160 & GAA & GAAGAAGAAGAAGAAGAA & 18 \\
\hline 133 & TGC & TGCTGCTGCTGCTGCTGC & 18 \\
\hline 180 & CTC & СТССТССТССТССТССТС & 18 \\
\hline & & & \\
\hline 143 & $\mathrm{AAG}$ & AAGAAGAAGAAGAAGAAGAAG & 21 \\
\hline 149 & CTC & СТССТССТССТССТССТССТС & 21 \\
\hline & & & \\
\hline 150 & CTT & СТTCTTCTTCTTCTTCTT & 18 \\
\hline 162 & TCT & TCTTCTTCTTCTTCTTCTTCTTCT & 24 \\
\hline & & & \\
\hline 148 & $\mathrm{AAC}$ & AACAACAACAACAACAAC & 18 \\
\hline 145 & AGA & \begin{tabular}{|l} 
AGAAGAAGAAGAAGAAGA \\
\end{tabular} & 18 \\
\hline & & & \\
\hline 147 & GCA & GCAGCAGCAGCAGCAGCAGCA & 21 \\
\hline 147 & ССT & ССТССТССТССТССТССТ & 18 \\
\hline & & & \\
\hline 152 & TAT & TATTATTATTATTATTATTATTAT & 24 \\
\hline 153 & TAT & TATTATTATTATTATTATTATTAT & 24 \\
\hline
\end{tabular}


Locus_3601_Transcript_12/13_Confidence_0.607_Length_2230 Locus_3647 Transcript 17/28 Confidence 0.605 Length_1346 Locus_3647_Transcript_17/28_Confidence_0.605_Length_1346 Locus_3661_Transcript_5/6_Confidence_0.609_Length_1175

Locus 3661_Transcript 5/6 Confidence 0.609 Length 1175 Locus_3725_Transcript_3/7_Confidence_0.750_Length_2829 Locus 3725 Transcript 3/7 Confidence 0.750 Length 2829

Locus 3752 Transcript 31/48 Confidence 0.360 Length 3874 Locus_3752_Transcript_31/48_Confidence_0.360_Length_3874 Locus 3754 Transcript_6/19_Confidence_0.573_Length 909

Locus_3754_Transcript_6/19_Confidence_0.573_Length_909 Locus_3771_Transcript_26/28_Confidence_0.588_Length_1545 Locus_3771_Transcript_26/28_Confidence_0.588_Length_1545 Locus_3817_Transcript_10/24_Confidence_0.607_Length_1400 Locus 3817_Transcript_10/24_Confidence_0.607_Length_1400 Locus_3821_Transcript 71/75_Confidence_0.285 Length_2379 Locus_3821_Transcript_71/75_Confidence_0.285_Length_2379 Locus_3828_Transcript_8/18_Confidence_0.621_Length_1816 Locus 3828 Transcript 8/18 Confidence 0.621 Length 1816 Locus_3853_Transcript_27/28_Confidence_0.562_Length_2467 Locus 3853 Transcript_27/28 Confidence 0.562_Length_2467 Locus_3858_Transcript_35/49_Confidence_0.508_Length_1774 Locus_3858_Transcript_35/49_Confidence_0.508_Length_1774 Locus 3858 Transcript 35/49 Confidence 0.508 Length 1774 Locus_3858_Transcript_35/49_Confidence_0.508_Length_1774 Locus_3897_Transcript_20/27_Confidence_0.605_Length_3408 Locus 3897 Transcript 20/27 Confidence 0.605 Length 3408 Locus_3897_Transcript_20/27_Confidence_0.605_Length_3408 Locus 3897 Transcript 20/27 Confidence 0.605 Length 3408 Locus_3897_Transcript_20/27_Confidence_0.605_Length_3408 Locus_3897_Transcript_20/27_Confidence_0.605_Length_3408 Locus_3909_Transcript_9/23 Confidence 0.516 Length_854

Locus_3909_Transcript_9/23_Confidence_0.516_Length_854 Locus_3979_Transcript_10/13_Confidence_0.619_Length_2188 Locus 3979 Transcript 10/13 Confidence 0.619 Length 2188 Locus_4028_Transcript_50/58_Confidence_0.579_Length_5799 Locus_4028_Transcript 50/58_Confidence_0.579_Length_5799 Locus 4093 Transcript 41/96 Confidence 0.356 Length 1989 Locus_4093_Transcript_41/96_Confidence_0.356_Length_1989 Locus_4186_Transcript_21/73_Confidence_0.612_Length_3860 Locus_4186_Transcript_21/73_Confidence_0.612_Length_3860

\begin{tabular}{|c|c|c|c|c|c|c|c|}
\hline REVERSE & 54.7 & 28.57 & ACCAACATCAATTTACCAAAA & & & & \\
\hline FORWARD & 56.28 & 33.33 & TTTTTCTTGAATCCCATGAGT & 155 & TGC & TGCTGCTGCTGCTGCTGC & 18 \\
\hline REVERSE & 58.54 & 47.62 & ATGAGGAACTTGCAGGAAGAG & & & & \\
\hline FORWARD & 54.39 & 45.45 & GAAGTGTCTTCCTCTTCTCAGT & 148 & GAG & GAGGAGGAGGAGGAGGAGGAG & 21 \\
\hline REVERSE & 54.63 & 28.57 & CTCAAAATCTCCATTGAAAAA & & & & \\
\hline FORWARD & 56.47 & 52.38 & GAGAGAGATGGAGAGGGAGAT & 156 & GGA & GGAGGAGGAGGAGGAGGA & 18 \\
\hline REVERSE & 55.05 & 38.1 & TGTGCCTTGTAGTGATTTTCT & & & & \\
\hline FORWARD & 56.16 & 33.33 & AACTATTTGCCAATGAACCAT & 151 & GAA & GAAGAAGAAGAAGAAGAA & 18 \\
\hline REVERSE & 55.66 & 42.86 & GTTTAGTGGGATAAAGGCTTG & & & & \\
\hline FORWARD & 55.32 & 42.86 & AGTCTGAAGCTTCTTCTTGCT & 154 & TCC & ТССТССТССТССТССТССТССТСС & 24 \\
\hline REVERSE & 55.18 & 47.62 & CAATATAGAGGAGGAGCAGGT & & & & \\
\hline FORWARD & 54.96 & 36.36 & TCATGTAGAATGAGACCTTGAA & 148 & $\mathrm{AAC}$ & AACAACAACAACAACAACAACAAC & 24 \\
\hline REVERSE & 55.18 & 47.62 & CGCTGTATTATCTCTGGTGAG & & & & \\
\hline FORWARD & 55.67 & 47.62 & $\begin{array}{l}\text { GAAGTGAAGGTGGAGAAAGAG } \\
\end{array}$ & 140 & AGG & AGGAGGAGGAGGAGGAGGAGG & 21 \\
\hline REVERSE & 54.81 & 52.38 & GCTGTTACTAGAACCCTCCTC & & & & \\
\hline FORWARD & 54.76 & 50 & AGTCGCCTTTAGACAGTCAG & 173 & TCC & TCСТCСТCСТССТССТСC & 18 \\
\hline REVERSE & 55 & 33.33 & GGAACCAATAAATCACAATGA & & & & \\
\hline FORWARD & 55.3 & 42.86 & AAGGGAAGTACTTGTTGAAGC & 153 & $\mathrm{CAA}$ & CAACAACAACAACAACAA & 18 \\
\hline REVERSE & 55.41 & 42.86 & GAGAGCTTGGTTGAAGTCTTT & & & & \\
\hline FORWARD & 55.81 & 60 & $\begin{array}{l}\text { GAGTAGCGTAGGAGGAGGAG } \\
\end{array}$ & 284 & GGA & GGAGGAGGAGGAGGAGGAGGA & 21 \\
\hline REVERSE & 54.38 & 38.1 & AGCAAACACATATCGATTACC & & & & \\
\hline FORWARD & 54.77 & 33.33 & TCATTTGACATTTCAGAGGTT & 149 & TCT & TCTTCTTCTTCTTCTTCTTCT & 21 \\
\hline REVERSE & 55.58 & 52.38 & GTCTCGGGGAAGTAGTTAGTG & & & & \\
\hline FORWARD & 54.77 & 33.33 & TCATTTGACATTTCAGAGGTT & 149 & TCT & TCTTCTTCTTCTTCTTCTTCT & 21 \\
\hline REVERSE & 55.58 & 52.38 & GTCTCGGGGAAGTAGTTAGTG & & & & \\
\hline FORWARD & 55.73 & 42.86 & TCATGAAGAAGAGTCGCATAG & 151 & GAG & GAGGAGGAGGAGGAGGAG & 18 \\
\hline REVERSE & 55.14 & 42.86 & AATTGGAGCTCCTCAGTTATC & & & & \\
\hline FORWARD & 55.73 & 42.86 & TCATGAAGAAGAGTCGCATAG & 151 & GAG & GAGGAGGAGGAGGAGGAG & 18 \\
\hline REVERSE & 55.14 & 42.86 & AATTGGAGCTCCTCAGTTATC & & & & \\
\hline FORWARD & 55.47 & 42.86 & AATTGACCTCCTCCTCTAACA & 169 & TCC & ТССТССТССТССТССТССТСС & 21 \\
\hline REVERSE & 55 & 47.62 & $\begin{array}{l}\text { CGATCAGAGAAGCTGAGAGTA } \\
\end{array}$ & & & & \\
\hline FORWARD & 54.84 & 28.57 & $\begin{array}{l}\text { CAGCAAAATTAAGGAAATGA } \\
\end{array}$ & 155 & TTC & TTCTTCTTCTTCTTCTTCTTCTTC & 24 \\
\hline REVERSE & 55.22 & 33.33 & TTATAAAACATGGTGGAATCG & & & & \\
\hline FORWARD & 55.19 & 52.38 & GCTGGAGTACTCTCTCCATCT & 153 & TGC & TGCTGCTGCTGCTGCTGC & 18 \\
\hline REVERSE & 55.54 & 33.33 & ATGATGAAGCAGAAGGATTTT & & & & \\
\hline FORWARD & 55.18 & 42.86 & ССТTCССТTCTAGGATTAACA & 151 & TGG & TGGTGGTGGTGGTGGTGG & 18 \\
\hline REVERSE & 55.05 & 38.1 & ACCAGAGCACAAAAATCTGTA & & & & \\
\hline FORWARD & 55.09 & 33.33 & TCACTTTTTGATTGACACACA & 147 & CTT & CTTCTTCTTCTTCTTCTT & 18 \\
\hline REVERSE & 55.13 & 33.33 & AGAAAATTGAAGAATCCAAGG & & & & \\
\hline FORWARD & 54.82 & 28.57 & AAATTGTCAAGCAATTGTGAT & 149 & TTA & TTATTATTATTATTATTATTA & 21 \\
\hline REVERSE & 55.08 & 38.1 & TCTGGCATGTAAGGAAATCTA & & & & \\
\hline
\end{tabular}


Locus_4186_Transcript_21/73_Confidence_0.612_Length_3860 Locus_4186_Transcript_21/73_Confidence_0.612_Length_3860 Locus_4282_Transcript_17/34_Confidence_0.623_Length_3440 Locus_4282_Transcript_17/34_Confidence_0.623_Length_3440 Locus 4282_Transcript 17/34 Confidence 0.623 Length 3440 Locus_4282_Transcript_17/34_Confidence_0.623_Length_3440 Locus_4321_Transcript_8/25_Confidence_0.544_Length_2935 Locus 4321 Transcript $8 / 25$ Confidence 0.544 Length 2935 Locus_4321_Transcript_8/25_Confidence_0.544_Length_2935 Locus 4321 Transcript $8 / 25$ Confidence 0.544 Length 2935 Locus_4323_Transcript_6/9_Confidence_0.619_Length_2915 Locus_4323_Transcript_6/9_Confidence_0.619_Length_2915 Locus_4323_Transcript_6/9_Confidence_0.619_Length_2915 Locus_4323_Transcript_6/9_Confidence_0.619_Length_2915 Locus_4323_Transcript_6/9_Confidence_0.619_Length_2915 Locus 4323 Transcript 6/9_Confidence_0.619 Length_2915 Locus_4342_Transcript_36/45_Confidence_0.611_Length_2646 Locus 4342 Transcript 36/45 Confidence 0.611 Length 2646 Locus_4356_Transcript_27/35_Confidence_0.594_Length_3718 Locus_4356_Transcript_27/35_Confidence_0.594_Length_3718 Locus_4375_Transcript_4/9_Confidence_0.675_Length_1663 Locus_4375_Transcript_4/9_Confidence_0.675_Length_1663 Locus_4403_Transcript_23/24_Confidence_0.558_Length_1213 Locus 4403 Transcript 23/24 Confidence 0.558 Length 1213 Locus_4410_Transcript_32/60_Confidence_0.374_Length_4715 Locus_4410_Transcript_32/60_Confidence_0.374_Length_4715 Locus 4444 Transcript $8 / 16$ Confidence 0.536 Length 2224 Locus_4444_Transcript_8 8116 CConfidence_0.536_Length_2224 Locus 4585 Transcript 24/28 Confidence 0.622 Length 3769 Locus 4585 Transcript 24/28 Confidence 0.622 Length 3769 Locus_4585_Transcript_24/28_Confidence_0.622_Length_3769 Locus_4585_Transcript_24/28_Confidence_0.622_Length_3769 Locus_4644_Transcript_20/25_Confidence_0.604_Length_2555 Locus_4644_Transcript_20/25_Confidence_0.604_Length_2555 Locus 4738 Transcript 98/111 Confidence 0.419 Length 2232 Locus_4738_Transcript_98/111_Confidence_0.419_Length_2232 Locus_4746_Transcript_21/30_Confidence_0.599_Length_2853 Locus 4746 Transcript 21/30 Confidence 0.599 Length 2853 Locus_4754_Transcript_34/54_Confidence_0.536_Length_1939 Locus_4754_Transcript_34/54_Confidence_0.536_Length_1939 Locus_4766_Transcript_9/14_Confidence_0.698_Length_4334

\begin{tabular}{|c|c|c|c|c|c|c|}
\hline FORWARD & 54.82 & \begin{tabular}{l|l}
28.57 & AAATTGTCAAGCAATTGTGAT \\
\end{tabular} & & TTA & TTATTATTATTATTATTATTA & 21 \\
\hline REVERSE & 55.08 & \begin{tabular}{l|l|l|}
38.1 & CTGGCATGAAGGAAATCTA \\
\end{tabular} & & & & \\
\hline FORWARD & 57.27 & \begin{tabular}{l|l|} 
50 & CTATGCTTCCCACAGAAACC \\
\end{tabular} & 213 & AGA & AGAAGAAGAAGAAGAAGAAGAAGA & 24 \\
\hline REVERSE & 55.33 & 50 CTGTGATCCACCATCCTTAC & & & & \\
\hline FORWARD & 55.45 & \begin{tabular}{l|l}
38.1 & TGGATCACAGGGTGATATAAA \\
\end{tabular} & 152 & AGA & AGAAGAAGAAGAAGAAGAAGA & 21 \\
\hline REVERSE & 57.2 & 50 CCATCTCCATTCCTTGCTAC & & & & \\
\hline FORWARD & 55.32 & \begin{tabular}{l|l}
38.1 & AATAATTCTGAGGGCAGAAG \\
\end{tabular} & 166 & TTA & TTATTATTATTATTATTATTATTA & 24 \\
\hline REVERSE & 54.99 & $\begin{array}{l}28.57 \\
\text { TGGAACCAAACAAAGATTAAA }\end{array}$ & & & & \\
\hline FORWARD & 55.47 & 38.1 CTCAATTGTTGTGATGCTCTT & 151 & ATT & ATTATTATTATTATTATTATTATT & 24 \\
\hline REVERSE & 55.1 & $\begin{array}{l}42.86 \\
\text { TGATCGTAAGTTCAGGAGAGA }\end{array}$ & & & & \\
\hline FORWARD & 55.03 & \begin{tabular}{l|l|} 
42.86ATTGGGACTCTACATAGC \\
\end{tabular} & 158 & TCA & TCATCATCATCATCATCATCA & 21 \\
\hline REVERSE & 54.92 & \begin{tabular}{l|l|} 
42.86AGTCCATCAGTCCAGAAA \\
\end{tabular} & & & & \\
\hline FORWARD & 54.92 & 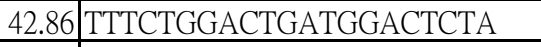 & 135 & TCA & TCATCATCATCATCATCATCA & 21 \\
\hline REVERSE & 54.86 & \begin{tabular}{l|l|l}
38.1 & TAATGAAGATGAGGGATGA \\
\end{tabular} & & & & \\
\hline FORWARD & 55.03 & 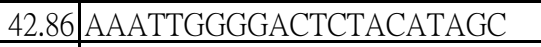 & 158 & TCA & TCATCATCATCATCATCATCA & 21 \\
\hline REVERSE & 54.92 & 42.86 TAGAGTCCATCAGTCCAGAAA & & & & \\
\hline FORWARD & 55.55 & \begin{tabular}{l|l}
38.1 & CTTCCTCCGTTATTTCAATTC \\
\end{tabular} & 173 & $\mathrm{CCG}$ & $\mathrm{CCG}$ & 18 \\
\hline REVERSE & 54.89 & \begin{tabular}{l|l|}
45 & AACTCCGGATCCTTATTCTC \\
\end{tabular} & & & & \\
\hline FORWARD & 54.52 & 42.86 CTATTGTGAAGAAGGAGGTGA & 152 & $\mathrm{AAG}$ & AAGAAGAAGAAGAAGAAGAAGAAG & 24 \\
\hline REVERSE & 54.7 & \begin{tabular}{|l|l|} 
42.86TTACTAACCTGCGTTGAT \\
\end{tabular} & & & & \\
\hline FORWARD & 54.89 & \begin{tabular}{l|l|l}
47.62 & GGAAGAAGAAGAGAGTGG \\
\end{tabular} & 170 & GAG & GAGGAGGAGGAGGAGGAGGAG & 21 \\
\hline REVERSE & 55.63 & \begin{tabular}{l|l|l}
38.1 & TAGCAGCGATTAACTTCTCAA \\
\end{tabular} & & & & \\
\hline FORWARD & 55.26 & 38.1 GACGAAGAAGATGATGATGAA & 190 & GGA & GGAGGAGGAGGAGGAGGA & 18 \\
\hline REVERSE & 54.78 & 38.1 TGGGCAAGTACATAAGAAAAG & & & & \\
\hline FORWARD & 54.54 & \begin{tabular}{l|l|l}
47.62 & TTCTATAGTCAGCCTCCACAC \\
\end{tabular} & 148 & TAT & TATTATTATTATTATTAT & 18 \\
\hline REVERSE & 55.18 & \begin{tabular}{|c|c|}
33.33 & TTATTGGCTACAAAGAAA \\
\end{tabular} & & & & \\
\hline FORWARD & 54.96 & \begin{tabular}{l|l|l}
38.1 & AGATTGATGACATGAGAATGG \\
\end{tabular} & 165 & AGA & AGAAGAAGAAGAAGAAGA & 18 \\
\hline REVERSE & 55.75 & 42.86 CCTCGAAGAATGAAAGAAGAC & & & & \\
\hline FORWARD & 54.51 & \begin{tabular}{l|l|}
42.86 & TATCATCATCACTGTCCTCCT \\
\end{tabular} & 149 & TCC & ТССТССТССТССТССТСС & 18 \\
\hline REVERSE & 55.42 & 42.86 AGAAATTGGCGGTAGAGTAAG & & & & \\
\hline FORWARD & 55.01 & \begin{tabular}{l|l}
42.86 & ATCCTTTCTGTCTTCCTTGTC \\
\end{tabular} & 160 & TCC & ТССТССТССТССТССТССТССТСС & 24 \\
\hline REVERSE & 54.63 & \begin{tabular}{l|l|}
42.86 & CCAATTCTTGCTGTACAGAGT \\
\end{tabular} & & & & \\
\hline FORWARD & 55.46 & \begin{tabular}{ll|l}
52.38 & GGAGACCTAGTCTCTCAATGG \\
\end{tabular} & 140 & TCT & TCTTCTTCTTCTTCTTCT & 18 \\
\hline REVERSE & 55.5 & \begin{tabular}{l|l|l|}
38.1 & GATCTCGTGCTTTTTCTTCTT \\
\end{tabular} & & & & \\
\hline FORWARD & 55.62 & \begin{tabular}{l|l}
47.62 & TACCTCGCCTATCTCCACTAT \\
\end{tabular} & 131 & AAT & AATAATAATAATAATAATAAT & 21 \\
\hline REVERSE & 54.01 & $\begin{array}{l}42.86 \text { GATGATCTAGTACGCAAATCC } \\
\end{array}$ & & & & \\
\hline FORWARD & 55.22 & \begin{tabular}{l|l}
38.1 & AACTGATGTCATCGAATTGAG \\
\end{tabular} & 175 & TAT & TATTATTATTATTATTATTATTAT & 24 \\
\hline REVERSE & 54.95 & 36.36 AGGGTCATAAGCAAGATAAAGA & & & & \\
\hline FORWARD & 55.16 & 42.86 ATACTAGTGGGTGGGGATAAA & 152 & TTA & TTATTATTATTATTATTATTA & 21 \\
\hline REVERSE & 54.93 & 42.86 GGTATGGAGGAAGGAGAATTA & & & & \\
\hline FORWARD & 54.98 & 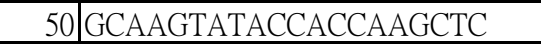 & 220 & CTC & СТССТССТССТССТССТССТС & 21 \\
\hline
\end{tabular}


Locus_4766_Transcript_9/14_Confidence_0.698_Length_4334 Locus 4825 Transcript 13/24 Confidence 0.607 Length 1069 Locus_4825_Transcript_13/24_Confidence_0.607_Length_1069 Locus_5009_Transcript_45/50_Confidence_0.587_Length_2313 Locus 5009 Transcript_45/50_Confidence_0.587_Length 2313 Locus_5019_Transcript_11/106_Confidence_0.346_Length_2575 Locus_5019_Transcript_11/106_Confidence_0.346_Length_2575 Locus 5026 Transcript $17 / 40$ Confidence 0.512 Length 2477 Locus_5026_Transcript_17/40_Confidence_0.512_Length_2477 Locus 5026 Transcript $17 / 40$ Confidence 0.512 Length 2477 Locus_5026_Transcript_17/40_Confidence_0.512_Length_2477 Locus_5026_Transcript_17/40_Confidence_0.512_Length_2477 Locus 5026_Transcript_17/40_Confidence_0.512_Length_2477 Locus_5112_Transcript_18/57_Confidence_0.343_Length_3924 Locus 5112_Transcript 18/57_Confidence_0.343 Length_3924 Locus 5135 Transcript 7/12 Confidence 0.640 Length 1727 Locus_5135_Transcript_7/12_Confidence_0.640_Length_172 Locus 5195 Transcript $7 / 8$ Confidence 0.705 Length 2732 Locus_5195_Transcript_7/8_Confidence_0.705_Length_2732 Locus_5207_Transcript_5/15_Confidence_0.614_Length_1161 Locus 5207 Transcript 5/15 Confidence 0.614 Length 1161 Locus_5313_Transcript_7/12_Confidence_0.736_Length_3452 Locus_5313_Transcript_7/12_Confidence_0.736_Length_3452 Locus 5343 Transcript 4/7 Confidence 0.643 Length 1275 Locus_5343_Transcript_4/7_Confidence_0.643_Length_1275 Locus 5362_Transcript_31/46_Confidence_0.596_Length_6554 Locus 5362 Transcript 31/46 Confidence 0.596 Length 6554 Locus_5364_Transcript_17/29_Confidence_0.592_Length_6988 Locus_5364_Transcript_17/29_Confidence_0.592_Length_6988 Locus_5370_Transcript_25/48_Confidence_0.579 Length 2130 Locus_5370_Transcript_25/48_Confidence_0.579_Length_2130 Locus 5442 Transcript 13/39 Confidence 0.553 Length 2271 Locus_5442_Transcript_13/39_Confidence_0.553_Length_2271 Locus_5512_Transcript_29/57_Confidence_0.534_Length_2884 Locus 5512 Transcript 29/57 Confidence 0.534 Length 2884 Locus_5512_Transcript_29/57_Confidence_0.534_Length_2884 Locus_5512_Transcript 29/57 Confidence 0.534 Length 2884 Locus 5594 Transcript 10/24 Confidence 0.455 Length 1278 Locus_5594_Transcript_10/24_Confidence_0.455_Length_1278 Locus_5594_Transcript_10/24_Confidence_0.455_Length_1278 Locus_5594_Transcript_10/24_Confidence_0.455_Length_1278

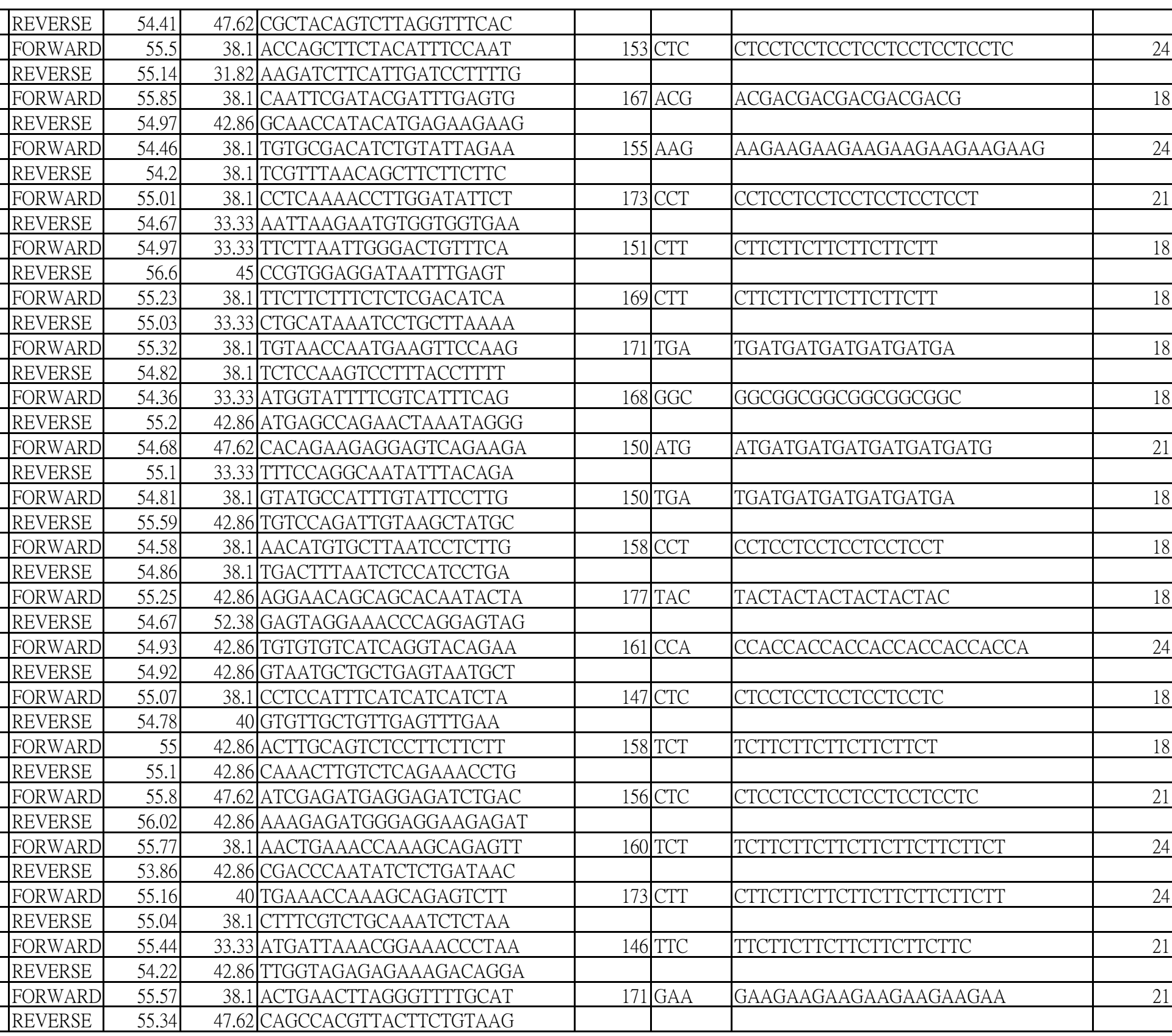


Locus_5613_Transcript_10/13_Confidence_0.358_Length_1444 Locus_5613_Transcript_10/13_Confidence_0.358_Length 1444 Locus_5622_Transcript_14/22_Confidence_0.630_Length_1717 Locus_5622_Transcript_14/22_Confidence_0.630_Length_1717 Locus 5627 Transcript 11/24 Confidence 0.629 Length 2835 Locus_5627_Transcript_11/24_Confidence_0.629_Length_2835 Locus_5680_Transcript_11/53_Confidence_0.475_Length_600 Locus 5680 Transcript 11/53 Confidence 0.475 Length 600 Locus_5746_Transcript_38/47_Confidence_0.645_Length_4236 Locus 5746 Transcript 38/47 Confidence 0.645 Length 4236 Locus_5810_Transcript_16/22_Confidence_0.621_Length_1695 Locus 5810_Transcript 16/22_Confidence_0.621_Length_1695 Locus_5836_Transcript_9/16_Confidence_0.635_Length_2136 Locus_5836_Transcript_9/16_Confidence_0.635_Length_2136 Locus_5843_Transcript_4/15_Confidence_0.656_Length_1635 Locus_ 5843 Transcript_4/15 Confidence_0.656_Length_1635 Locus_5844_Transcript_7/23_Confidence_0.598_Length_2089 Locus 5844_Transcript 7/23_Confidence 0.598_Length 2089 Locus_5853_Transcript_10/28_Confidence_0.664_Length_2945 Locus_5853_Transcript_10/28_Confidence_0.664_Length_2945 Locus 5891 Transcript 20/28 Confidence 0.603 Length 2255 Locus_5891_Transcript_20/28_Confidence_0.603_Length_2255 Locus_5931_Transcript_12/22_Confidence_0.654_Length_1580 Locus 5931 Transcript 12/22 Confidence 0.654 Length 1580 Locus_5949_Transcript_14/56_Confidence_0.585_Length_5646 Locus_5949_Transcript_14/56_Confidence 0.585 Length 5646 Locus 5985 Transcript 6/7 Confidence 0.762 Length 1791 Locus_5985_Transcript_6/7_Confidence_0.762_Length_1791 Locus_5994_Transcript_13/31_Confidence_0.555_Length_2021 Locus 5994 Transcript 13/31 Confidence 0.555 Length 2021 Locus_6009_Transcript_5/12_Confidence_0.655_Length_1634 Locus 6009 Transcript $5 / 12$ Confidence 0.655 Length 1634 Locus_6048_Transcript_2/8_Confidence_0.724_Length_6756 Locus_6048_Transcript_2/8_Confidence_0.724_Length 6756 Locus 6052 Transcript 17/31 Confidence 0.578 Length 2060 Locus_6052_Transcript_17/31_Confidence_0.578_Length_2060 Locus_6071_Transcript_4/6_Confidence_0.690_Length_899 Locus 6071_Transcript 4/6 Confidence 0.690 Length 899 Locus_6095_Transcript_18/40_Confidence_0.498_Length_1644 Locus_6095_Transcript_18/40_Confidence_0.498_Length_1644 Locus_6138_Transcript_12/33_Confidence_0.633_Length_2617

\begin{tabular}{|c|c|c|c|c|c|c|c|}
\hline FORWARD & 54.95 & & ATCAAAACTCCATCTTTCTCC & 146 & TCT & TCTTCTTCTTCTTCTTCTTCTTCTTCTTCTTC & 36 \\
\hline REVERSE & 54.37 & 38.1 & TCTAATATACACACGGTGCAA & & & & \\
\hline FORWARD & 55.36 & 38.1 & TGCAGGTAAAAAGAGTTCAGA & 150 & CGC & CGCCGCCGCCGCCGCCGC & 18 \\
\hline REVERSE & 55.26 & 38.1 & AAGACGACTTTTTCCTTTGTC & & & & \\
\hline FORWARD & 54.99 & 33.33 & TGCAATCTTTCACAAACCTAT & 154 & $\mathrm{CAA}$ & CAACAACAACAACAACAA & 18 \\
\hline REVERSE & 54.75 & 28.57 & TTTGCATGATTTTGTTGTATG & & & & \\
\hline FORWARD & 54.93 & 47.62 & CAGATGGACCTATGCTATGAG & 149 & CTT & CTTCTTCTTCTTCTTCTTCTT & 21 \\
\hline REVERSE & 54.96 & 38.1 & TCTTAGTTTGTTCTGCTTTGG & & & & \\
\hline FORWARD & 54.52 & 38.1 & GATTATTGGGCTACAAAGTCA & 147 & AAT & AATAATAATAATAATAATAATAAT & 24 \\
\hline REVERSE & 54.96 & 38.1 & AGCGTGATATAGTTTTTGCTG & & & & \\
\hline FORWARD & 51.72 & 40 & AGGTATACAAGGAATCATGC & 129 & AAT & AATAATAATAATAATAAT & 18 \\
\hline REVERSE & 54.54 & 47.62 & GTGAGGAGAGCTTGTATGGTA & & & & \\
\hline FORWARD & 54.95 & 38.1 & CCTTTGTTCTCATTTCATCAG & 198 & $\mathrm{AAG}$ & AAGAAGAAGAAGAAGAAG & 18 \\
\hline REVERSE & 55.48 & 38.1 & GATGAAGAAATGTTCCATGTG & & & & \\
\hline FORWARD & 54.28 & 38.1 & AGTGTCCAGATTAAAGAGCAA & 145 & TCA & TCATCATCATCATCATCATCA & 21 \\
\hline REVERSE & 54.48 & 42.86 & CTTAAAGCTAGGGGATGATCT & & & & \\
\hline FORWARD & 54.86 & 38.1 & TAATTTGAAGCACTCTTCGTC & 149 & AGA & AAGA & 24 \\
\hline REVERSE & 54.67 & 38.1 & CAGACAATAAAAACACCCATC & & & & \\
\hline FORWARD & 55 & 38.1 & CACATCCAAATCTTCGTAAAG & 147 & TCT & TCTTCTTCTTCTTCTTCT & 18 \\
\hline REVERSE & 55.02 & 38.1 & ATGATATTGGTCTCCCTTCAT & & & & \\
\hline FORWARD & 56.38 & 38.1 & TGCTGAAGCAGAAAGTTCATA & 191 & GGC & GGCGGCGGCGGCGGCGGCGGC & 21 \\
\hline REVERSE & 55.29 & 47.62 & GTGGAAGCCTTGTCTCTACTT & & & & \\
\hline FORWARD & 54.99 & 40 & TGTTTCGTCCTCAGAAGTTT & 148 & TCT & TTCT & 18 \\
\hline REVERSE & 54.89 & 36.36 & CAAGTAAACGAAGAAGACAACA & & & & \\
\hline FORWARD & 55.01 & 38.1 & CCCATCTTCAGGTTTCTTATT & 139 & CTG & CTGCTGCTGCTGCTGCTGCTGCTG & 24 \\
\hline REVERSE & 54.93 & 35 & ACAAAATCATGCCATATTCC & & & & \\
\hline FORWARD & 55.53 & 47.62 & GTATTTTAGTGGCGGACCTAC & 134 & GGC & GGCGGCGGCGGCGGCGGCGGC & 21 \\
\hline REVERSE & 54.81 & 38.1 & AAAGGATTTAGTACCGCTCAT & & & & \\
\hline FORWARD & 55.08 & 38.1 & TGAGTAGGAGAATATGGCAAA & 178 & AGA & AGAAGAAGAAGAAGAAGAAGAAGA & 24 \\
\hline REVERSE & 55.06 & 38.1 & TGATTCTTCTTCTTCTGGTGA & & & & \\
\hline FORWARD & 55.04 & 47.62 & GACTTCCTGCTCTTTCTCTTC & 152 & ATC & ATCATCATCATCATCATC & 18 \\
\hline REVERSE & 54.32 & 38.1 & GACTTTGAATCTCGTTGTGAT & & & & \\
\hline FORWARD & 54.92 & 42.86 & ATGGAAGAGTTTCCTGATCTC & 153 & CTT & CTTCTTCTTCTTCTTCTT & 18 \\
\hline REVERSE & 55.3 & 38.1 & ACAGGAACCAATTTCTCTCAT & & & & \\
\hline FORWARD & 54.78 & 47.62 & GTGTACATCTCTTCCTCATCG & 146 & GAA & GAAGAAGAAGAAGAAGAA & 18 \\
\hline REVERSE & 55.18 & 42.86 & CCCTTTCTGTCTCATCTTCTT & & & & \\
\hline FORWARD & 55.63 & 38.1 & AGATTTGGGTTCAATCATCTC & 164 & CAC & CACCACCACCACCACCAC & 18 \\
\hline REVERSE & 55.18 & 47.62 & TGGTGGAGTTGGTAGTGATAG & & & & \\
\hline FORWARD & 54.75 & 42.86 & CTGTATTTAGCAGGCTTTACG & 122 & GAA & GAAGAAGAAGAAGAAGAAGAAGAA & 24 \\
\hline REVERSE & 55.6 & 42.86 & GCTGGTTTAACAGCTTCTTCT & & & & \\
\hline FORWARD & 55.18 & 42.86 & CAATTCCTTCCTCTTCACTCT & 151 & $\mathrm{CCA}$ & CCACCACCACCACCACCACCACCA & 24 \\
\hline
\end{tabular}


Locus_6138_Transcript_12/33_Confidence_0.633_Length_2617 Locus_6175_Transcript_13/15_Confidence_0.605_Length_1116 Locus_6175_Transcript_13/15_Confidence_0.605_Length_1116 Locus_6222_Transcript_28/90_Confidence_0.394_Length_1403 Locus 6222 Transcript 28/90 Confidence 0.394 Length 1403 Locus_6223_Transcript_2/4_Confidence_0.846_Length_1056 Locus 6223 Transcript 2/4 Confidence 0.846 Length 1056 Locus 6223 Transcript 2/4 Confidence 0.846 Length 1056 Locus_6223_Transcript_2/4_Confidence_0.846_Length_1056 Locus 6238 Transcript $2 / 12$ Confidence 0.598 Length 1434 Locus_6238_Transcript_2/12_Confidence_0.598_Length_1434 Locus_6266_Transcript_6/12_Confidence_0.642_Length_1038 Locus_6266_Transcript_6/12_Confidence_0.642_Length_1038 Locus_6315_Transcript_24/36_Confidence_0.555_Length_1383 Locus_6315_Transcript_24/36_Confidence_0.555_Length_1383 Locus_6363_Transcript_13/22_Confidence_0.619_Length_4555 Locus_6363_Transcript_13/22_Confidence_0.619_Length_4555 Locus_6408_Transcript 32/64_Confidence_0.513 Length_3008 Locus_6408_Transcript 32/64 Confidence_0.513_Length_3008 Locus_6472_Transcript_22/39_Confidence_0.527_Length_3761 Locus 6472 Transcript 22/39 Confidence 0.527 Length 3761 Locus_6547_Transcript_16/21_Confidence_0.646_Length_2117 Locus_6547_Transcript_16/21_Confidence_0.646_Length_2117 Locus 6559 Transcript $48 / 60$ Confidence 0.470 Length 8761 Locus_6559_Transcript_48/60_Confidence_0.470_Length_8761 Locus_6604_Transcript_6/7_Confidence_0.724_Length_1387

Locus 6604 Transcript 6/7 Confidence 0.724 Length 1387 Locus_6635_Transcript_24/32_Confidence_0.585_Length_1789 Locus 6635_Transcript 24/32 Confidence 0.585 Length 1789 Locus 6635 Transcript 24/32 Confidence 0.585 Length 1789 Locus_6635_Transcript_24/32_Confidence_0.585_Length_1789 Locus_6635_Transcript_24/32_Confidence_0.585_Length_1789 Locus_6635_Transcript_24/32_Confidence_0.585_Length_1789 Locus_6681_Transcript_60/94_Confidence_0.567_Length_2134 Locus 6681 Transcript 60/94 Confidence 0.567 Length 2134 Locus_6745_Transcript_8/8_Confidence_0.561_Length_631 Locus_6745 Transcript 8/8 Confidence 0.561 Length 631 Locus 6745 Transcript $8 / 8$ Confidence 0.561 Length 631 Locus_6745_Transcript_8/8_Confidence_0.561_Length_631 Locus_6785_Transcript_16/34_Confidence_0.602_Length_1881 Locus_6785_Transcript_16/34_Confidence_0.602_Length_1881

\begin{tabular}{|c|c|c|c|c|c|c|}
\hline REVERSE & 54.67 & \begin{tabular}{l|l|}
33.33 & GAATGAAAGTTTTCGAACAGA \\
\end{tabular} & & & & \\
\hline FORWARD & 55.62 & \begin{tabular}{l|l|l}
38.1 & TTTACTATCCTGGCGGTAT \\
\end{tabular} & 139 & GGC & GGCGGCGGCGGCGGCGGCGGC & 21 \\
\hline REVERSE & 55.46 & \begin{tabular}{l|l|l}
47.62 & GACAGGTTCGAGAGGTAATTC \\
\end{tabular} & & & & \\
\hline FORWARD & 55.04 & 42.86|CGACCCTTCTATTTGGTCTAT & 181 & CTC & СТССТССТССТССТССТС & 18 \\
\hline REVERSE & 55 & \begin{tabular}{l|l|}
45 & GATGCCATCATCATCTCTCT \\
\end{tabular} & & & & \\
\hline FORWARD & 55.72 & \begin{tabular}{l|l}
47.62 & TTCACAGTCGTTACTCG \\
\end{tabular} & 198 & $\mathrm{GCA}$ & GCAGCAGCAGCAGCAGCAGCA & 21 \\
\hline REVERSE & 55.27 & \begin{tabular}{l|l|l}
42.86 & AAGGAGTTATGTAGCAAGG \\
\end{tabular} & & & & \\
\hline FORWARD & 54.16 & $\begin{array}{l}42.86 \\
\text { TCCTTTCTCACAGTCGTTACT } \\
\end{array}$ & 150 & $\mathrm{GCA}$ & GCAGCAGCAGCAGCAGCAGCA & 21 \\
\hline REVERSE & 55.09 & \begin{tabular}{|l|l|}
33.33 & AATTATTCGAAATCACTGC \\
\end{tabular} & & & & \\
\hline FORWARD & 55.04 & \begin{tabular}{l|l|l}
47.62 & GTCTTGAGGTAGTCGGAGAT \\
\end{tabular} & 129 & $\mathrm{CCG}$ & CCGCCGCCGCCGCCGCCGCCG & 21 \\
\hline REVERSE & 55.11 & 50 GACGCAGCCGATATACTAAC & & & & \\
\hline FORWARD & 55.45 & \begin{tabular}{l|l|}
50 & TCTTACTCCACACCTGAAGC \\
\end{tabular} & 220 & $\mathrm{CCG}$ & CCGCCGCCGCCGCCGCCGCCG & 21 \\
\hline REVERSE & 54.23 & \begin{tabular}{l|l}
38.1 & ATGACGATATTTCATCCTCTG \\
\end{tabular} & & & & \\
\hline FORWARD & 55.18 & \begin{tabular}{l|l|l}
47.62 & GAGGAGGAAGAAGTAGA \\
\end{tabular} & 150 & GAA & GAAGAAGAAGAAGAAGAAGAA & 21 \\
\hline REVERSE & 55.1 & \begin{tabular}{l|l|l|}
42.86 & GAGCTATGGCATCAATGTAAG \\
\end{tabular} & & & & \\
\hline FORWARD & 54.61 & 45 ACCTTTGTTAACACGCTCTC & 153 & TTC & TTCTTCTTCTTCTTCTTC & 18 \\
\hline REVERSE & 54.93 & \begin{tabular}{l|l|}
28.57 & AATCAAGCAAAATATGGTGA \\
\end{tabular} & & & & \\
\hline FORWARD & 54.82 & 42.86|GTCGGTGAAGCAAGTAACTAA & 165 & CTC & СТССТССТССТССТССТС & 18 \\
\hline REVERSE & 55.15 & \begin{tabular}{l|l|}
42.86 & GTATCAAGGGGAGATCATC \\
\end{tabular} & & & & \\
\hline FORWARD & 55.52 & \begin{tabular}{|l|c|}
42.86 & CTCTTGTACCACGAACGATA \\
\end{tabular} & 179 & AAC & AACAACAACAACAACAAC & 18 \\
\hline REVERSE & 54.93 & 38.1 GGTATTGTGGTTGTTGTTGTT & & & & \\
\hline FORWARD & 55.06 & \begin{tabular}{|l|l|}
42.86 & GCTTGAAGAATACGCTGTAGA \\
\end{tabular} & 146 & $\mathrm{GAG}$ & GAGGAGGAGGAGGAGGAGGAG & 21 \\
\hline REVERSE & 55.23 & 42.86 TTCTTCATCTTTCCCTCTCTC & & & & \\
\hline FORWARD & 54.45 & \begin{tabular}{l|l}
33.33 & ATTGAACAACACCTGATGAT \\
\end{tabular} & 146 & TGA & TGATGATGATGATGATGA & 18 \\
\hline REVERSE & 54.72 & 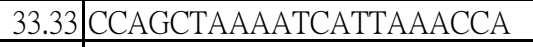 & & & & \\
\hline FORWARD & 55.14 & 38.1 TCAAAGTTCTCCTTGTCTTCA & 161 & TTC & TTCTTCTTCTTCTTCTTC & 18 \\
\hline REVERSE & 54.8 & \begin{tabular}{|l|l|}
42.86 & GTGGTAATCCGAGATCCTAAT \\
\end{tabular} & & & & \\
\hline FORWARD & 55.25 & \begin{tabular}{l|l|} 
47.62 & AGCTACAGTTACCGAGGATTC \\
\end{tabular} & 140 & GAT & GATGATGATGATGATGAT & 18 \\
\hline REVERSE & 54.82 & 42.86 GTCGATTTCTTCTTCTTCCTC & & & & \\
\hline FORWARD & 55.01 & \begin{tabular}{l|l}
33.33 & AAAAATCGAGGAAGAGAAGA \\
\end{tabular} & 129 & GAT & GATGATGATGATGATGAT & 18 \\
\hline REVERSE & 54.68 & \begin{tabular}{|l|l}
33.33 & TTTTAGATTTTGGATCCCTCT \\
\end{tabular} & & & & \\
\hline FORWARD & 55.25 & \begin{tabular}{l|l|l|} 
47.62 & AGCTACAGTACCGAGGATTC \\
\end{tabular} & 140 & GAT & GATGATGATGATGATGAT & 18 \\
\hline REVERSE & 54.82 & 42.86 GTCGATTTCTTCTTCTTCCTC & & & & \\
\hline FORWARD & 55.04 & \begin{tabular}{|l|l|}
33.33 & GATTTGCTTAAACAGCTTTA \\
\end{tabular} & 158 & ATC & ATCATCATCATCATCATC & 18 \\
\hline REVERSE & 54.94 & $\begin{array}{ll}33.33 & \text { AGGTTGAAAGGAAGAAGAAAA } \\
\end{array}$ & & & & \\
\hline FORWARD & 55.05 & \begin{tabular}{|l|c|}
42.86 & CTCAACAAATAGAGCACACC \\
\end{tabular} & 219 & $\mathrm{GAG}$ & GAGGAGGAGGAGGAGGAGGAGGAG & 24 \\
\hline REVERSE & 56.3 & 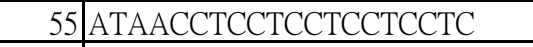 & & & & \\
\hline FORWARD & 55.49 & \begin{tabular}{l|l}
50 & AAGAAGAAGCAGAGGAGGAG \\
\end{tabular} & 102 & GAG & GAGGAGGAGGAGGAGGAG & 18 \\
\hline REVERSE & 56.17 & \begin{tabular}{|l|l|}
38.1 & AACTCGACGACAAAAACTTTC \\
\end{tabular} & & & & \\
\hline FORWARD & 55.07 & \begin{tabular}{l|l|l}
47.62 & TCGGATCTCTCTCCTATTACC \\
\end{tabular} & 144 & TGC & TGCTGCTGCTGCTGCTGCTGC & 21 \\
\hline REVERSE & 55.17 & 38.1 AAAGTGGGTAAGGAAAACAAG & & & & \\
\hline
\end{tabular}


Locus_6786_Transcript_3/26_Confidence_0.534_Length_1862 Locus_6786_Transcript_3/26_Confidence_0.534_Length_1862 Locus_6799_Transcript_8/24_Confidence_0.595_Length_2930 Locus_6799_Transcript_8/24_Confidence_0.595_Length_2930 Locus 6808 Transcript 11/23 Confidence 0.609 Length 2763 Locus_6808_Transcript_11/23_Confidence_0.609_Length_2763 Locus 6841_Transcript_15/25_Confidence_0.523_Length 4256 Locus 6841 Transcript $15 / 25$ Confidence 0.523 Length 4256 Locus_6852_Transcript_8/13_Confidence_0.633_Length_5223 Locus 6852 Transcript $8 / 13$ Confidence 0.633 Length 5223 Locus_6878_Transcript_14/16_Confidence_0.641_Length_2274 Locus_6878_Transcript_14/16_Confidence_0.641_Length_2274 Locus 6879_Transcript_28/29_Confidence_0.631_Length_2738 Locus_6879_Transcript_28/29_Confidence_0.631_Length_2738 Locus_6947_Transcript_8/14_Confidence_0.633_Length_1806 Locus 6947 Transcript 8/14 Confidence 0.633 Length 1806 Locus_6964_Transcript_13/48_Confidence_0.635_Length_1667 Locus 6964 Transcript $13 / 48$ Confidence 0.635 Length 1667 Locus_7086_Transcript_4/9_Confidence_0.686_Length_596 Locus_7086_Transcript_4/9_Confidence_0.686_Length_596 Locus 7119 Transcript 6/9 Confidence 0.706 Length 1661 Locus_7119_Transcript_6/9_Confidence_0.706_Length_1661 Locus_7119_Transcript_6/9_Confidence_0.706_Length_1661 Locus 7119 Transcript 6/9 Confidence 0.706 Length 1661 Locus_7139_Transcript_12/14_Confidence_0.600_Length_1290 Locus 7139 Transcript 12/14 Confidence 0.600 Length 1290 Locus 7183 Transcript 2/8 Confidence 0.571 Length 665 Locus_7183_Transcript_2/8_Confidence_0.571_Length_665 Locus 7251 Transcript_12/29 Confidence 0.433 Length_1559 Locus 7251 Transcript 12/29 Confidence 0.433 Length 1559 Locus_7279_Transcript_15/40_Confidence_0.289_Length_1748 Locus 7279 Transcript 15/40 Confidence 0.289 Length 1748 Locus_7280_Transcript_3/5_Confidence_0.875_Length_1604 Locus_7280_Transcript_3/5_Confidence_0.875_Length_1604 Locus 7282 Transcript 33/41 Confidence 0.525 Length 2349 Locus_7282_Transcript_33/41_Confidence_0.525_Length_2349 Locus_7291_Transcript 13/15 Confidence 0.589 Length 1688 Locus 7291 Transcript 13/15 Confidence 0.589 Length 1688 Locus_7304_Transcript_13/31_Confidence_0.457_Length_719 Locus_7304_Transcript_13/31_Confidence_0.457_Length_719 Locus_7330_Transcript_23/24_Confidence_0.638_Length_1973

\begin{tabular}{|c|c|c|c|c|c|c|c|}
\hline FORWARD & 55.39 & & ATCCAATACCCAACCATTTAC & 153 & TCT & TCTTCTTCTTCTTCTTCT & 18 \\
\hline REVERSE & 55.16 & 38.1 & AATTAAACCAGAATCGAGAGG & & & & \\
\hline FORWARD & 55.05 & 33.33 & TTAGAAAAGATGGTTCGATGA & 152 & TGT & TGTTGTTGTTGTTGTTGTTGT & 21 \\
\hline REVERSE & 54.86 & 38.1 & ACCATTACCAATAACAGAGCA & & & & \\
\hline FORWARD & 55.22 & 42.86 & AGCATAGAAGGAAAGTCCAAC & 162 & TGA & TGATGATGATGATGATGA & 18 \\
\hline REVERSE & 55.27 & 38.1 & CACCTTTTCTTCCTTTCAACT & & & & \\
\hline FORWARD & 56.38 & 47.62 & ССТСТСАСССССТАAСТАAАТ & 172 & CCT & ССТССТССТССТССТССТ & 18 \\
\hline REVERSE & 55.6 & 40.91 & CCATGAACTGAGGAATATAAGC & & & & \\
\hline FORWARD & 54.91 & 42.86 & AGAGATGATAGAGGGATTTGG & 154 & GAG & GAGGAGGAGGAGGAGGAGGAG & 21 \\
\hline REVERSE & 54.38 & 42.86 & ACCTAAGGCGGTATATTTCTC & & & & \\
\hline FORWARD & 54.62 & 38.1 & TCTACAATTTCTGAGGATTGC & 152 & CCT & ССТССТССТССТСТССТ & 18 \\
\hline REVERSE & 54.99 & 33.33 & ACAGAAGCAAATGGTGAAATA & & & & \\
\hline FORWARD & 55.37 & 47.62 & GTAACTGACGAACCTGTGAGA & 161 & $\mathrm{GCC}$ & GCCGCCGCCGCCGCCGCC & 18 \\
\hline REVERSE & 54.14 & 40 & CCAAATCCTCTCATAAAAGC & & & & \\
\hline FORWARD & 55.51 & 38.1 & GGAAAAACAGAAAACAGGAAC & 149 & TAA & TAATAATAATAATAATAA & 18 \\
\hline REVERSE & 55.39 & 38.1 & GAAGCCATCACTTTAATCACA & & & & \\
\hline FORWARD & 54.7 & 38.1 & GTAGCCTTGTGAGAAAATCAA & 153 & $\mathrm{AAG}$ & AAGAAGAAGAAG & 27 \\
\hline REVERSE & 54.93 & 38.1 & CCATCGTTTAACAGCTAAGAA & & & & \\
\hline FORWARD & 55.29 & 45.45 & CGTTCTCTCTTCTTATCTCTCG & 157 & TTA & TTATTATTATTATTATTATTATTA & 24 \\
\hline REVERSE & 54.59 & 38.1 & AGGGGGATTCAATGTAATATC & & & & \\
\hline FORWARD & 54.77 & 38.1 & TTAACTTTTAGGCACGTCATC & 153 & TTG & TTGTTGTTGTTGTTGTTG & 18 \\
\hline REVERSE & 55.05 & 33.33 & AACAACAACAACAACAAATCC & & & & \\
\hline FORWARD & 55.11 & 42.86 & CACACATAGTGGGATTAAGGA & 146 & TTG & TTGTTGTTGTTGTTGTTG & 18 \\
\hline REVERSE & 54.74 & 33.33 & TTGAAGAAATACACCTTTTCG & & & & \\
\hline FORWARD & 55.42 & 38.1 & AAGAAGAAGAAGAGGGGTTTT & 155 & GAG & GAGGAGGAGGAGGAGGAG & 18 \\
\hline REVERSE & 55.29 & 28.57 & AAAAATCAAATCGGAAAAGAG & & & & \\
\hline FORWARD & 54.1 & 45 & CAGCATCCTTAGGGTTTTAC & 152 & CGG & CGGCGGCGGCGGCGGCGG & 18 \\
\hline REVERSE & 55.78 & 47.62 & CTTCCCTACTGAGAGCAAATC & & & & \\
\hline FORWARD & 55.59 & 38.1 & GGCTTGCTTAATGTTGATGTA & 132 & GAT & GATGATGATGATGATGATGAT & 21 \\
\hline REVERSE & 55.77 & 38.1 & ATCAACAACATCATCATCGTC & & & & \\
\hline FORWARD & 54.56 & 38.1 & CTTGATAACGCCATCATAGTT & 160 & TTC & TTCTTCTTCTTCTTCTTC & 18 \\
\hline REVERSE & 55.51 & 38.1 & CAAGCCAAAGAAGAAGAAGAT & & & & \\
\hline FORWARD & 59.79 & 40 & ACAAACCGCAAAGCAAAACT & 175 & TTC & TTCTTCTTCTTCTTCTTC & 18 \\
\hline REVERSE & 57 & 40.91 & GGTTTGTGGATATTGTTGAGTG & & & & \\
\hline FORWARD & 54.82 & 42.86 & CTACATTGAGAATCACCCTTG & 159 & ATA & ATAATAATAATAATAATA & 18 \\
\hline REVERSE & 54.65 & 28.57 & TGCTTCTATAAAATTGCGTTT & & & & \\
\hline FORWARD & 54.39 & 47.62 & TGTGTCTCCTACTCTTCAACC & 157 & TTC & TTCTTCTTCTTCTTCTTC & 18 \\
\hline REVERSE & 55.18 & 33.33 & AGCAAAAAGTGAATCAGTCAA & & & & \\
\hline FORWARD & 54.76 & 42.86 & TGTAGCTTCTTTTCAGGTGAC & 187 & GCG & GCGGCGGCGGCGGCGGCGGCGGCG & 24 \\
\hline REVERSE & 53.75 & 31.82 & CTTTGTCAAATTCTAAAACTGC & & & & \\
\hline FORWARD & 56.93 & 33.33 & ATCCATCATTGCGTTATCAAT & 145 & $\mathrm{GAC}$ & GACGACGACGACGACGAC & 18 \\
\hline
\end{tabular}


Locus_7330_Transcript_23/24_Confidence_0.638_Length_1973 Locus_7358 Transcript 11/19 Confidence 0.361 Length 2039 Locus_7358_Transcript_11/19_Confidence_0.361_Length_2039 Locus_7381_Transcript_10/26_Confidence_0.615_Length_2685 Locus 7381_Transcript_10/26_Confidence_0.615_Length_2685 Locus_7414_Transcript_36/37_Confidence_0.586_Length_2435 Locus_7414_Transcript_36/37_Confidence_0.586_Length_2435 Locus 7426 Transcript 9/20 Confidence 0.638 Length 2976 Locus_7426_Transcript_9/20_Confidence_0.638_Length_2976 Locus 7426 Transcript 9/20 Confidence 0.638 Length 2976 Locus_7426_Transcript_9/20_Confidence_0.638_Length_2976 Locus_7519_Transcript_15/46_Confidence_0.603_Length_1387 Locus 7519 Transcript 15/46 Confidence 0.603 Length 1387 Locus_7679_Transcript_20/21_Confidence_0.586_Length_1272 Locus 7679_Transcript 20/21_Confidence_0.586_Length_1272 Locus 7693 Transcript 21/31 Confidence 0.602 Length 3346 Locus_7693_Transcript_21/31_Confidence_0.602_Length_3346 Locus 7717 Transcript_ $7 / 13$ Confidence 0.597 Length 1519 Locus 7717_Transcript 7/13 Confidence 0.597 Length_1519 Locus_7769_Transcript_17/58_Confidence_0.528_Length_1302 Locus 7769 Transcript $17 / 58$ Confidence 0.528 Length 1302 Locus_7797_Transcript_5/12_Confidence_0.530_Length_1068 Locus_7797_Transcript_5/12_Confidence_0.530_Length_1068 Locus 7798_Transcript 16/33 Confidence_0.327_Length 1309 Locus_7798_Transcript_16/33_Confidence_0.327_Length_1309 Locus_7807_Transcript_17/19_Confidence_0.603_Length_1588 Locus 7807 Transcript 17/19 Confidence 0.603 Length 1588 Locus_7846_Transcript_21/42_Confidence_0.541_Length_1987 Locus_7846_Transcript_21/42_Confidence_0.541_Length_1987 Locus_7848_Transcript_6/22_Confidence_0.462_Length_1523 Locus_7848_Transcript_6/22_Confidence_0.462_Length_1523 Locus 7852 Transcript $9 / 29$ Confidence 0.503 Length 936 Locus_7852_Transcript_9/29_Confidence_0.503_Length_936 Locus 7862 Transcript 17/29 Confidence 0.592 Length 2303 Locus 7862 Transcript 17/29 Confidence 0.592 Length 2303 Locus_7879_Transcript_6/15_Confidence_0.627_Length_2840 Locus_7879_Transcript_6/15 Confidence 0.627 Length 2840 Locus 7907 Transcript 33/39 Confidence 0.489 Length 2945 Locus_7907_Transcript_33/39_Confidence_0.489_Length_2945 Locus_7909_Transcript_15/21_Confidence_0.538_Length_2474 Locus_7909_Transcript_15/21_Confidence_0.538_Length_2474

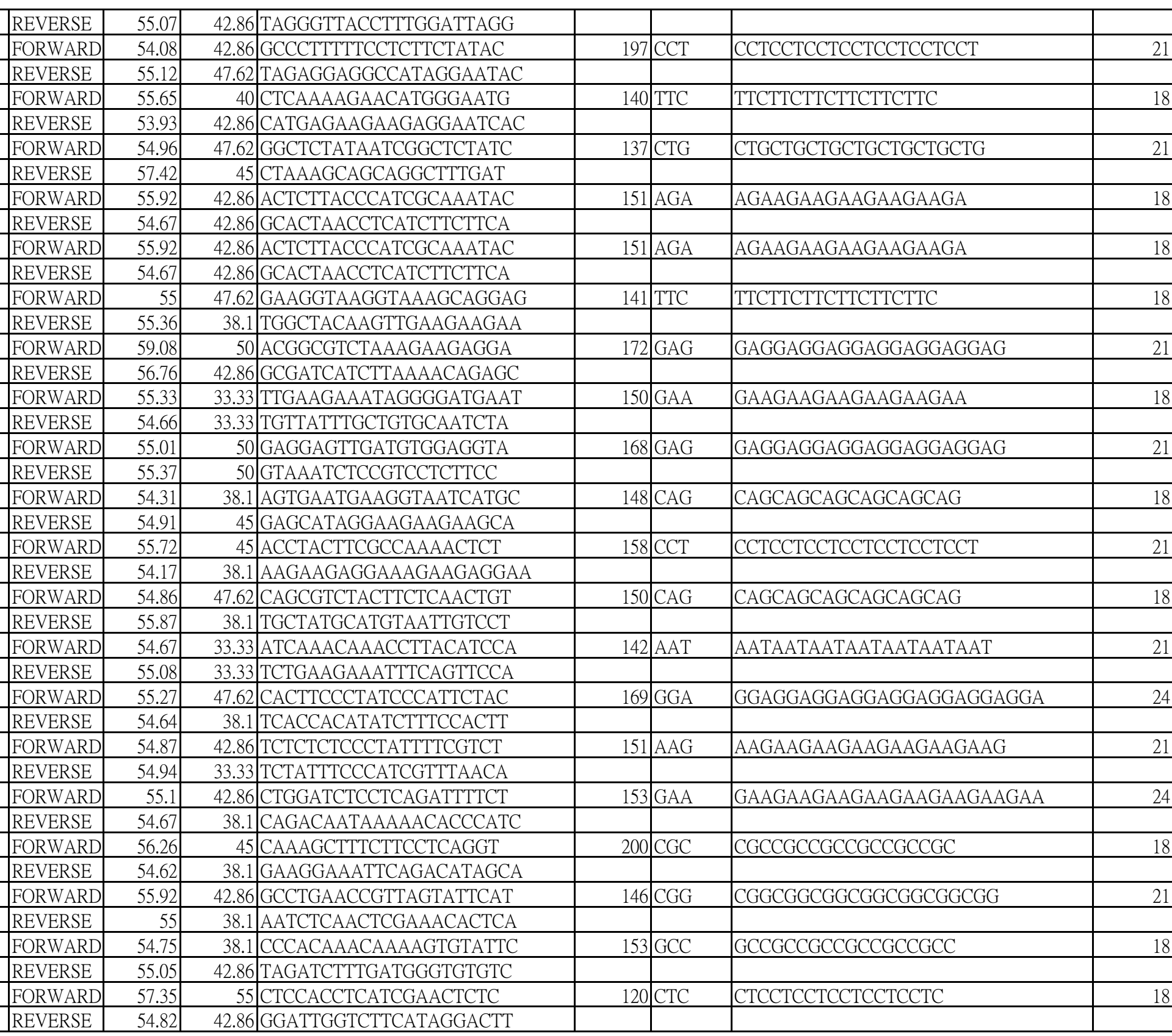


Locus_7928_Transcript_14/15_Confidence_0.590_Length_1182 Locus_7928_Transcript 14/15 Confidence 0.590 Length_1182 Locus_7956_Transcript_23/40_Confidence_0.444_Length_1735 Locus_7956_Transcript_23/40_Confidence_0.444_Length_1735 Locus 8081 Transcript 1/9 Confidence _0.644 Length 2237 Locus_8081_Transcript_1/9_Confidence_0.644_Length_2237 Locus_8081_Transcript_1/9_Confidence_0.644_Length_2237 Locus 8081 Transcript $1 / 9$ Confidence 0.644 Length 2237 Locus_8104_Transcript_15/28_Confidence_0.557_Length_1784 Locus 8104 Transcript 15/28 Confidence 0.557 Length 1784 Locus_8119_Transcript_23/30_Confidence_0.497_Length_787 Locus_8119_Transcript_23/30_Confidence_0.497_Length_787 Locus_8126_Transcript_16/21_Confidence_0.643_Length_916 Locus_8126_Transcript_16/21_Confidence_0.643_Length_916 Locus_8130_Transcript_17/28_Confidence_0.593_Length_2985 Locus 8130 Transcript 17/28 Confidence 0.593 Length 2985 Locus_8225_Transcript_5/9_Confidence_0.758_Length_2188 Locus 8225 Transcript 5/9 Confidence 0.758 Length 2188 Locus_8236_Transcript_2/10_Confidence_0.609_Length_619 Locus_8236_Transcript_2/10_Confidence_0.609_Length_619 Locus 8236 Transcript 2/10_Confidence_0.609 Length 619 Locus_8236_Transcript_2/10_Confidence_0.609_Length_619 Locus_8347_Transcript_17/25_Confidence_0.595_Length_1741 Locus 8347 Transcript 17/25 Confidence 0.595 Length 1741 Locus_8425_Transcript_6/17_Confidence_0.542_Length_5486 Locus_8425_Transcript_6/17_Confidence_0.542_Length_5486 Locus 8425 Transcript 6/17 Confidence 0.542 Length 5486 Locus_8425_Transcript_6/17_Confidence_0.542_Length_5486 Locus 8425 Transcript 6/17 Confidence 0.542 Length 5486 Locus 8425 Transcript 6/17 Confidence 0.542 Length 5486 Locus_8425_Transcript_6/17_Confidence_0.542_Length_5486 Locus_8425_Transcript_6/17_Confidence_0.542_Length_5486 Locus_8704_Transcript_7/12_Confidence_0.615_Length_1013 Locus_8704_Transcript_7/12_Confidence 0.615 Length_1013 Locus 8728 Transcript $2 / 8$ Confidence 0.690 Length 2219 Locus_8728_Transcript_2/8_Confidence_0.690_Length_2219 Locus 8740 Transcript 2/14 Confidence 0.492 Lenoth 789 Locus 8740 Transcript 2/14 Confidence 0.492 Length 789 Locus_8789_Transcript_2/13_Confidence_0.588_Length_1083 Locus_8789_Transcript_2/13_Confidence_0.588_Length_1083 Locus_8793_Transcript_15/15_Confidence_0.553_Length_2240

\begin{tabular}{|c|c|c|c|c|c|c|}
\hline FORWARD & 54.65 & \begin{tabular}{l|l|l} 
42.86 & ATAAGAGGAAGAAGGCTCAAG \\
\end{tabular} & 151 & TCC & ТССТССТССТССТССТССТСС & 21 \\
\hline REVERSE & 55.65 & $\begin{array}{l}\text { 42.86 } \\
\text { CATGATGTCGAGATGAGTC } \\
\end{array}$ & & & & \\
\hline FORWARD & 55.01 & 38.1 AAGAATCCCTCATTAAACCAG & 166 & CTT & СTTCTTCTTCTTCTTCTTCTT & 21 \\
\hline REVERSE & 55.01 & 38.1 TCATAATCTGCTTTAGGCTTG & & & & \\
\hline FORWARD & 54.67 & \begin{tabular}{l|l|l|}
50 & CCTAAGAGTCTGACGATGCT \\
\end{tabular} & 223 & $\mathrm{AGG}$ & AGGAGGAGGAGGAGGAGG & 18 \\
\hline REVERSE & 55.43 & 33.33 AGCATAAACCCATCATTTTCT & & & & \\
\hline FORWARD & 55.43 & 33.33 AGAAAATGATGGGTTTATGCT & 286 & AGG & AGGAGGAGGAGGAGGAGG & 18 \\
\hline REVERSE & 56.18 & \begin{tabular}{l|l}
50 & TACAACCTCСТCСТCТТСТ \\
\end{tabular} & & & & \\
\hline FORWARD & 54.3 & 28.57|AAGCAAATTTCTGCACATTAT & 155 & TCT & TCTTCTTCTTCTTCTTCT & 18 \\
\hline REVERSE & 54.92 & \begin{tabular}{|l|l|}
38.1 & GATTTAAAGTTGGGGACAGT \\
\end{tabular} & & & & \\
\hline FORWARD & 54.51 & $\begin{array}{l}47.62 \text { GGTCTCCTTGTTCACATACAC } \\
\end{array}$ & 138 & CTT & CTTCTTCTTCTTCTTCTTCTTCTT & 24 \\
\hline REVERSE & 55.01 & $\begin{array}{l}\text { 42.86 } \\
\text { CAAGGACAGAAGACATTGAG }\end{array}$ & & & & \\
\hline FORWARD & 54.86 & $\begin{array}{l}33.33 \text { GAAGCACCTAAAATAATGCAA } \\
\end{array}$ & 160 & AAT & AATAATAATAATAATAATAAT & 21 \\
\hline REVERSE & 54.92 & 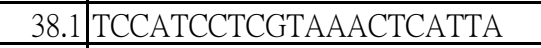 & & & & \\
\hline FORWARD & 55.24 & \begin{tabular}{l|l|} 
ATCTCTCTGCAACGAAAGAA \\
\end{tabular} & 149 & CTT & СTTCTTCTTCTTCTTCTTCTT & 21 \\
\hline REVERSE & 55.04 & 33.33 ACAATACGCAAAATACCAAGA & & & & \\
\hline FORWARD & 55.01 & 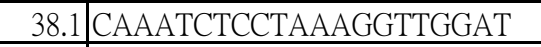 & 149 & TCC & TCСТCСТC & 18 \\
\hline REVERSE & 55 & \begin{tabular}{l|l}
38.1 & GAAAAGGATGTAACTCGGATT \\
\end{tabular} & & & & \\
\hline FORWARD & 55.19 & 38.1 TCATCTATTCCTGCTCTTTGA & 140 & CCT & ССТССТССТССТССТССТ & 18 \\
\hline REVERSE & 54.92 & \begin{tabular}{l|l|l|} 
42.8GGAGGAGAAATGAATCAGAC \\
\end{tabular} & & & & \\
\hline FORWARD & 54.16 & \begin{tabular}{l|l|}
47.62 & GATAAACCCTAGTGAGTGCTG \\
\end{tabular} & 154 & CTC & СТССТССТССТССТССТССТС & 21 \\
\hline REVERSE & 54.92 & $\begin{array}{l}\text { 42.86 } \\
\text { AGGAGGAGAAATGATCAGAC } \\
\end{array}$ & & & & \\
\hline FORWARD & 55.23 & 42.86 GGGACTAAAAATCATCACCTC & 168 & TCA & TCATCATCATCATCATCA & 18 \\
\hline REVERSE & 55.11 & 42.86 ACAGATTCAAGACACCTGATG & & & & \\
\hline FORWARD & 54.59 & 38.1 TGAAGAGCTTACTGAAAGGAA & 144 & TTC & TTCTTCTTCTTCTTCTTCTTCTTC & 24 \\
\hline REVERSE & 55.22 & \begin{tabular}{l|l|l|} 
42.8AAGAAGAAGAAACGAAGC \\
\end{tabular} & & & & \\
\hline FORWARD & 54.59 & 38.1 TGAAGAGCTTACTGAAAGGAA & 135 & TTC & TTCTTCTTCTTCTTCTTCTTCTTC & 24 \\
\hline REVERSE & 55.7 & 33.33 TAACAACAACAAAAACGAAGC & & & & \\
\hline FORWARD & 55.7 & \begin{tabular}{|l|l|}
33.33 & GCTTCGTTTTTGTTGTTGTA \\
\end{tabular} & 159 & TTC & TTCTTCTTCTTCTTCTTC & 18 \\
\hline REVERSE & 56.75 & \begin{tabular}{l|l|}
35 & AATGAGCTTTCGCAAGAAAT \\
\end{tabular} & & & & \\
\hline FORWARD & 54.34 & \begin{tabular}{ll|l|l|}
31.82 & TTTGTTGAGATTAATGCTCTGT \\
\end{tabular} & 149 & TTC & TTCTTCTTCTTCTTCTTC & 18 \\
\hline REVERSE & 55.67 & 45.45 СССТСТСТССАТCCTTATAACT & & & & \\
\hline FORWARD & 55.87 & 38.1 GGGGTTGTTGAAAAGATTAGA & 150 & TCT & TCTTCTTCTTCTTCTTCT & 18 \\
\hline REVERSE & 55.01 & $\begin{array}{l}\text { 42.86 } \\
\text { AGAACAAGAACAGGGAGATTC } \\
\end{array}$ & & & & \\
\hline FORWARD & 55.02 & 42.86 AGTTGACACACAGAACCAATC & 148 & CGG & CGGCGGCGGCGGCGGCGG & 18 \\
\hline REVERSE & 55.36 & 38.1 AATAAGCTCGGGAGTATTTTG & & & & \\
\hline FORWARD & 54.73 & 38.1 GCCACCTTTATTAGAAAATCC & 159 & GAA & GAAGAAGAAGAAGAAGAAGAAGAA & 24 \\
\hline REVERSE & 55.09 & \begin{tabular}{l|l}
47.62 & TTAAGCTCTCСТСАСТССТСТ \\
\end{tabular} & & & & \\
\hline FORWARD & 54.62 & 38.1 ACGGAATATCCACTCATGTTA & 140 & GCG & GCGGCGGCGGCGGCGGCG & 18 \\
\hline REVERSE & 55.23 & 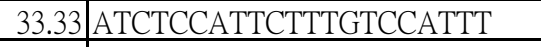 & & & & \\
\hline FORWARD & 54.84 & 33.33|CTGAAGAAAATCTTCGAAACA & 159 & CCT & |ССТССТССТССТССТССТССТ & 21 \\
\hline
\end{tabular}


Locus_8793_Transcript_15/15_Confidence_0.553_Length_2240 Locus_8826_Transcript_7/17_Confidence_0.597_Length_817 Locus_8826_Transcript_7/17_Confidence_0.597_Length_817 Locus_8843_Transcript_4/26_Confidence_0.654_Length_2211 Locus 8843 Transcript 4/26 Confidence 0.654 Length 2211 Locus_8843_Transcript_4/26_Confidence_0.654_Length_2211 Locus_8843_Transcript_4/26_Confidence_0.654_Length_2211 Locus 8959 Transcript 22/28 Confidence 0.622 Length 2579 Locus_8959_Transcript_22/28_Confidence_0.622_Length_2579 Locus 8964_Transcript_22/39_Confidence_0.597_Length_2344 Locus_8964_Transcript_22/39_Confidence_0.597_Length_2344 Locus_8964_Transcript_22/39_Confidence_0.597_Length_2344 Locus_8964_Transcript_22/39_Confidence_0.597_Length_2344 Locus_9032_Transcript_32/34_Confidence_0.557_Length_2832 Locus_9032_Transcript 32/34_Confidence_0.557 Length_2832 Locus 9057 Transcript 4/10 Confidence 0.729 Length 3372 Locus_9057_Transcript_4/10_Confidence_0.729_Length_3372 Locus 9096 Transcript 17/19 Confidence 0.670 Lenoth 1388 Locus 9096 Transcript 17/19_Confidence_0.670_Length 1388 Locus_9101_Transcript_29/42_Confidence_0.592_Length_1655 Locus_9101_Transcript_29/42_Confidence_0.592_Length_1655 Locus_9197_Transcript_5/7_Confidence_0.435_Length_693 Locus_9197_Transcript 5/7_Confidence_0.435_Length_693 Locus_9197_Transcript 5/7_Confidence_0.435_Length 693 Locus_9197_Transcript_5/7_Confidence_0.435_Length_693 Locus 9197 Transcript 5/7 Confidence 0.435 Length 693 Locus 9197 Transcript 5/7 Confidence 0.435 Length 693 Locus_9245_Transcript_28/53_Confidence_0.583_Length_1841 Locus_9245_Transcript_28/53_Confidence_0.583_Length_1841 Locus 9397 Transcript 19/28 Confidence 0.594 Length 1395 Locus_9397_Transcript_19/28_Confidence_0.594_Length_1395 Locus 9502 Transcript $8 / 19$ Confidence 0.651 Length 1315 Locus_9502_Transcript_8/19_Confidence_0.651_Length_1315 Locus_9647_Transcript_11/15_Confidence_0.600_Length_602 Locus 9647 Transcript $11 / 15$ Confidence 0.600 Length 602 Locus_9706_Transcript_4/11_Confidence_0.667_Length_594 Locus_9706_Transcript 4/11 Confidence 0.667 Length 594 Locus 9706 Transcript 4/11 Confidence 0.667 Length 594 Locus_9706_Transcript_4/11_Confidence_0.667_Length_594 Locus_9722_Transcript_13/14_Confidence_0.704_Length_515 Locus_9722_Transcript_13/14_Confidence_0.704_Length_515

\begin{tabular}{|c|c|c|c|c|c|c|c|}
\hline REVERSE & 54.32 & 33.33 & AAAGAAACCAGCTTGTGATTA & & & & \\
\hline FORWARD & 54.91 & & ATATCGTTTCAACTGCTGTGT & 158 & TTA & TTATTATTATTATTATTA & 18 \\
\hline REVERSE & 54.75 & 24 & TGCAAATAAAAAGTAAACTACAAGA & & & & \\
\hline FORWARD & 54.97 & 42.86 & AACCACTAACCTTATCCCAAG & 154 & TCC & ТССТССТССТССТССТССТССТСС & 24 \\
\hline REVERSE & 55.67 & 47.62 & TAAGCTTCTTCAGAGGAGGAG & & & & \\
\hline FORWARD & 54.64 & 33.33 & CCAAAATCTCCAATAGCATTA & 154 & TCC & TССТССТССТССТССТССТССТСС & 24 \\
\hline REVERSE & 54.58 & 33.33 & TTGGTGAATGTTTCCTATGAT & & & & \\
\hline FORWARD & 54.94 & 45.45 & CCGTACTCCTTACTCTTTTGTC & 147 & CCT & ССТССТССТССТСТССТ & 18 \\
\hline REVERSE & 54.91 & 42.86 & TGAATGAGCTCGTAGTAAAGG & & & & \\
\hline FORWARD & 54.8 & 38.1 & GTTCAGATGAGAAGAAAGCAA & 147 & $\mathrm{AAG}$ & AAGAAGAAGAAGAAGAAGAAGAAGAAG & 27 \\
\hline REVERSE & 55.07 & 33.33 & TTGGTTGCACAGTTTTTCTAT & & & & \\
\hline FORWARD & 55.35 & 42.86 & TCAGGATTCTGTTCAGATGAG & 147 & GAA & GAAGAAGAAGAAGAAGAAGAAGAA & 24 \\
\hline REVERSE & 55.07 & 33.33 & TTGGTTGCACAGTTTTTCTAT & & & & \\
\hline FORWARD & 55.15 & 42.86 & CCATGAAACAGATAAGCAGAG & 148 & AGA & AGAAGAAGAAGAAGAAGA & 18 \\
\hline REVERSE & 55.49 & 42.86 & AGCGTTACTTGTTCACACAAC & & & & \\
\hline FORWARD & 54.96 & 42.86 & AAAGAGCATACTGCACAGAAG & 148 & $\mathrm{ACC}$ & ACCACCACCACCACCACC & 18 \\
\hline REVERSE & 54.85 & 33.33 & ACCAGTTCATCAAAGTTGAAA & & & & \\
\hline FORWARD & 55.12 & 28.57 & CCCAAAAAGGTTTTAATTTGT & 162 & ATT & ATTATTATTATTATTATT & 18 \\
\hline REVERSE & 54.94 & 42.86 & CAGAGAGCTTGAAGTTGGTAA & & & & \\
\hline FORWARD & 55.59 & 38.1 & ACTCCAAATATCATCGGATTC & 202 & GGC & CGGC & 18 \\
\hline REVERSE & 55.64 & 42.86 & TTCTCTTGATGTCGAAGTACG & & & & \\
\hline FORWARD & 54.82 & 38.1 & GAATTAGCCAATTTCTCTTCC & 149 & CTC & СТССТССТССТССТССТС & 18 \\
\hline REVERSE & 55.92 & 47.62 & GAGAAGGAGATGAGCAAGAAG & & & & \\
\hline FORWARD & 55.18 & 42.86 & CTTCTTCCTTCTGGTTCATCT & 148 & CTC & СТССТССТССТССТССТС & 18 \\
\hline REVERSE & 55.23 & 42.86 & TATGATGAAGTGGAGGTTGAG & & & & \\
\hline FORWARD & 53.8 & 38.1 & TCGTCTACATTCTGAACATTG & 151 & CTC & СТССТССТССТССТССТС & 18 \\
\hline REVERSE & 55.71 & 42.86 & ATGAACCAGAAGGAGATGAAC & & & & \\
\hline FORWARD & 54.57 & 42.86 & GGGTTACTCCTCAATTTCTTC & 117 & $\mathrm{CAG}$ & CAGCAGCAGCAGCAGCAG & 18 \\
\hline REVERSE & 54.76 & 38.1 & TTCATCTGGTTAACCTCCATA & & & & \\
\hline FORWARD & 54.67 & 38.1 & AAGAGATCAAAGCATTGACAG & 138 & TTC & TTCTTCTTCTTCTTCTTC & 18 \\
\hline REVERSE & 55.21 & 33.33 & GCCAATGATGTAAATTTGGTA & & & & \\
\hline FORWARD & 54.72 & 33.33 & TCATCCACCATAGCAATAAAT & 152 & TAT & TATTATTATTATTATTAT & 18 \\
\hline REVERSE & 56.1 & 42.86 & AAATAAAGAGAGGTCGGGAGT & & & & \\
\hline FORWARD & 56.87 & 50 & ATATAGTCAGGCTGCGATCC & 151 & CCG & CCGCCGCCGCCGCCGCCGCCGCCG & 24 \\
\hline REVERSE & 59.5 & 40 & TTCCCAATGGCTCTTGAAAT & & & & \\
\hline FORWARD & 54.59 & 45 & AAGGATGCTTTACGTACGAC & 219 & CGC & CGCCGCCGCCGCCGCCGC & 18 \\
\hline REVERSE & 54.81 & 45 & GTCATCATCTTCTTCCTTCG & & & & \\
\hline FORWARD & 56.96 & 50 & GAAGGAAGAATCACCACCAC & 131 & CGC & CGCCGCCGCCGCCGCCGC & 18 \\
\hline REVERSE & 56.44 & 38.1 & GAAAACCAGCCGTTAATTATG & & & & \\
\hline FORWARD & 55.35 & 42.86 & TGAAGATGAGATGGAGAGTTG & 144 & TTC & TTCTTCTTCTTCTTCTTCTTCTTC & 24 \\
\hline REVERSE & 54.9 & 33.33 & CACCTCAAAGCAATCTAAAAA & & & & \\
\hline
\end{tabular}


Locus_9722_Transcript_13/14_Confidence_0.704_Length_515 Locus_9722_Transcript_13/14_Confidence_0.704_Length_515 Locus_9769_Transcript_12/24_Confidence_0.556_Length_1914 Locus_9769_Transcript_12/24_Confidence_0.556_Length_1914 Locus_9835_Transcript_2/6_Confidence_0.667_Length_1538 Locus_9835_Transcript_2/6_Confidence_0.667_Length_1538 Locus 10098 Transcript 9/10 Confidence 0.607 Length 2122 Locus 10098 Transcript 9/10 Confidence 0.607 Length 2122 Locus_10151_Transcript_4/7_Confidence_0.776_Length_1997 Locus 10151 Transcript 4/7 Confidence 0.776 Length 1997 Locus_10204_Transcript_4/6_Confidence_0.775_Length_1334 Locus_10204_Transcript_4/6_Confidence_0.775_Length_1334 Locus_10236_Transcript_8/13_Confidence_0.579_Length_854 Locus_10236_Transcript_8/13_Confidence_0.579_Length_854 Locus_10311_Transcript_5/8_Confidence_0.615_Length_1555 Locus_10311_Transcript_5/8_Confidence_0.615_Length_1555 Locus_10349_Transcript_13/16_Confidence_0.647_Length_1660 Locus_10349_Transcript_13/16_Confidence_0.647_Length_1660 Locus_10349_Transcript 13/16 Confidence 0.647 Length 1660 Locus_10349_Transcript_13/16_Confidence_0.647_Length_1660 Locus 10415 Transcript 7/11 Confidence 0.548 Length 1005 Locus 10415 Transcript 7/11 Confidence 0.548 Length 1005 Locus_10454_Transcript_27/28_Confidence_0.568_Length_1300 Locus 10454 Transcript 27/28 Confidence 0.568 Length 1300 Locus_10519_Transcript_2/3_Confidence_0.778_Length_1629 Locus 10519 Transcript 2/3 Confidence 0.778 Length 1629

Locus 10585 Transcript 9/10 Confidence 0.634 Length 1058 Locus_10585_Transcript_9/10_Confidence_0.634_Length_1058 Locus 10594 Transcript 29/32 Confidence 0.576 Length 1473 Locus 10594 Transcript 29/32 Confidence 0.576 Length 1473 Locus_10627_Transcript_9/11_Confidence_0.625_Length_2738 Locus 10627 Transcript 9/11 Confidence 0.625 Length 2738 Locus_10665_Transcript_14/16_Confidence_0.592_Length_1869 Locus_10665_Transcript_14/16_Confidence_0.592_Length 1869 Locus 10668 Transcript 3/25 Confidence 0.531 Length 2174 Locus_10668_Transcript_3/25_Confidence_0.531_Length_2174 Locus_10694_Transcript_11/16_Confidence_0.595_Length_1920 Locus 10694 Transcript 11/16 Confidence 0.595 Length 1920 Locus_10694_Transcript_11/16_Confidence_0.595_Length_1920 Locus_10694_Transcript_11/16_Confidence_0.595_Length_1920 Locus_10710_Transcript_16/17_Confidence_0.578_Length_2306 \begin{tabular}{|l|r|r|r|}
\hline FORWARD & 55.08 & 38.1 & AATCCTCAGATTCCTATTTGC \\
\hline
\end{tabular} \begin{tabular}{lrr} 
REVERSE & 54.94 & 47.62 \\
\hline
\end{tabular} FORWARD REVERSE FORWARD REVERSE FORWARD REVERSE FORWARD REVERSE FORWARD REVERSE FORWARD REVERSE FORWARD REVERSE FORWARD REVERSE FORWARD REVERSE FORWARD REVERSE FORWARD REVERSE FORWARD REVERSE FORWARD REVERSE FORWARD REVERSE FORWARD REVERSE FORWARD REVERSE FORWARD REVERSE FORWARD REVERSE FORWARD REVERSE FORWARD $55.63 \quad 42.86$ ACCAGATCCAATCTCTGATTC \begin{tabular}{r|r|l}
54.37 & 38.1 & GTTTTCGCATTCTATATGCTC \\
\hline
\end{tabular} 55.07 45 ATGAAGGTATTCGGTGAGTG \begin{tabular}{r|r|r}
54.86 & 47.62 & GCACCTTATCTCTCTTCCTTC \\
\hline 55.05 & 38.1 & AGACACCTTAATGTTGCTGA \\
\hline
\end{tabular}

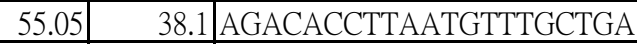
$55.05 \quad 38.1$ AAGTTTGTAGCAGTTCCATCA $54.43 \quad 42.86$ TATTAGTGGGGTGAACTCTTG 45 GTGCCCATCAGAAATAGTGT 42.86 CTCCCTCTGCTTGTTTACTTT 33.33 TAAGCCCCCATAATATTTCTT 33.33 AATCGGTACAGGCTTTAAAAT 42.86 CATCTTCCCCAATCTCTTATC 42.86 GCTCATCAGAACTATCATTGG 38.1 TTAAAAAGGAGGAGCTAAAGG 47.62 GGGATCACTAGACTCTCCAAT 47.62 ATCCTCGTACTCTTCTTCCAC 33.33 CATATTTGCCAAAGAAGGTTA 35 ATTTCTTCTTCATTGCAAGC 36.36 ACCAAGAAGGTTCTAGAAGAAA 42.86 CTATCAGCATCATTGTCCTTC 47.62 AGAGGGGTATGGTAATAGCTG 38.1 CATTCCTTGGTTATTTGTGTC 47.62 TCTCTATTCGTCGGTAGTCCT 33.33 TAAGATGTTCATTTGCTTGTG 33.33 TCTTGATCAGAAAAATCCTCA 33.33 AAAAAGGTTTGCAGTATGATG 50 CTTCAGTTCCTGTTCTCCTG 38.1 CCATCGTTTAACAGCTAAGAA 38.1 ATGCTGATTATGTTGCAGAAC 42.86 CACATTTCTTCTGGTTAGTGG 42.86 AGATCTCCGTCTCATCTTCAT 42.86 TACATGCCTTACCTTGAGAAG 38.1 TGCTCAGCAAATCTTAACTTC 38.1 CAGACAATAAAAACACCCATC 38.1 GAATTTGTTGATGACGAGAGA 42.86 ACCAGAAGAAGAAGAAGAAGC 50 CACTATGGTTCCCCTGTCTA 47.62 ACTCTCCTTCATCAACTCCTC 45 AGTTGAGCACAATCTTGACC

\begin{tabular}{|c|c|c|c|}
\hline 153 & CTT & CTTCTTCTTCTTCTTCTTCTTCTT & 24 \\
\hline 150 & GAA & GAAGAAGAAGAAGAAGAA & 18 \\
\hline 155 & GGT & GGTGGTGGTGGTGGTGGT & 18 \\
\hline & & & \\
\hline 204 & TAA & TAATAATAATAATAATAA & 18 \\
\hline 150 & GCC & GCCGCCGCCGCCGCCGCC & 18 \\
\hline & & & \\
\hline 164 & ATT & ATTATTATTATTATTATT & 18 \\
\hline 150 & AAG & AAGAAGAAGAAGAAGAAG & 18 \\
\hline 158 & CAT & CATCATCATCATCATCAT & 18 \\
\hline 144 & $\mathrm{GAG}$ & GAGGAGGAGGAGGAGGAG & 18 \\
\hline & & & \\
\hline 148 & ATG & ATGATGATGATGATGATG & 18 \\
\hline 140 & AGA & AGAAGAAGAAGAAGAAGAAGAAGA & 24 \\
\hline & & & \\
\hline 165 & AAT & AATAATAATAATAATAAT & 18 \\
\hline & & & \\
\hline 148 & TTA & TTATTATTATTATTATTATTA & 21 \\
\hline 149 & TCT & TCTTCTTCTTCTTCTTCTTCTTCT & 24 \\
\hline & & & \\
\hline 150 & $\mathrm{AAG}$ & AAGAAGAAGAAGAAGAAGAAG & 21 \\
\hline 152 & AGA & AGAAGAAGAAGAAGAAGA & 18 \\
\hline & & & \\
\hline 165 & GGC & GGCGGCGGCGGCGGCGGC & 18 \\
\hline 160 & AAG & AAGAAGAAGAAGAAGAAGAAGAAG & 24 \\
\hline & & & \\
\hline 145 & CTT & CTTCTTCTTCTTCTTCTT & 18 \\
\hline 1511 & |CTT & ICTTC TTCTTC & 18 \\
\hline 151 & C11 & 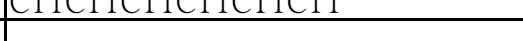 & 18 \\
\hline 143 & GCG & GCGGCGGCGGCGGCGGCGGCGGCG & 24 \\
\hline
\end{tabular}


Locus_10710_Transcript_16/17_Confidence_0.578_Length_2306 Locus_10737_Transcript_14/32_Confidence_0.552_Length_1297 Locus_10737_Transcript_14/32_Confidence_0.552_Length_1297 Locus_10816_Transcript_8/16_Confidence_0.671_Length_1024 Locus 10816 Transcript 8/16 Confidence_0.671 Length_1024 Locus_10874_Transcript_1/6_Confidence_0.632_Length_920 Locus_10874_Transcript_1/6_Confidence_0.632_Length_920

Locus 10936_Transcript_11/12 Confidence 0.649_Length_2074 Locus_10936_Transcript_11/12_Confidence_0.649_Length_2074 Locus 11004 Transcript 4/7 Confidence 0.667 Length 877

Locus_11004_Transcript_4/7_Confidence_0.667_Length_877 Locus_11033_Transcript_29/35_Confidence_0.407_Length_2500 Locus_11033_Transcript_29/35_Confidence_0.407_Length_2500 Locus_11132_Transcript_11/12_Confidence_0.652_Length_1500 Locus_11132_Transcript_11/12_Confidence_0.652_Length_1500 Locus_11388_Transcript_2/12_Confidence_0.573_Length_2001 Locus_11388_Transcript_2/12_Confidence_0.573_Length_2001 Locus_11388_Transcript_2/12_Confidence_0.573_Length_2001 Locus_11388_Transcript_2/12_Confidence_0.573_Length_2001 Locus_11397_Transcript_2/6_Confidence_0.625_Length_287 Locus 11397 Transcript 2/6 Confidence_0.625_Length_287 Locus_11442_Transcript_1/9_Confidence_0.648_Length_1057 Locus 11442 Transcript $1 / 9$ Confidence 0.648 Length 1057 Locus_11474_Transcript_1/4_Confidence_0.833_Length_912 Locus_11474_Transcript_1/4_Confidence_0.833_Length_912 Locus 11539 Transcript 3/13 Confidence 0.706 Length 850 Locus 11539 Transcript 3/13 Confidence 0.706 Length 850 Locus_11539_Transcript_3/13_Confidence_0.706_Length_850 Locus 11539 Transcript 3/13 Confidence 0.706 Length 850 Locus_11539_Transcript_3/13_Confidence_0.706_Length_850 Locus_11539_Transcript_3/13_Confidence_0.706_Length_850 Locus_11592_Transcript_3/4_Confidence_0.700_Length_651 Locus_11592_Transcript_3/4_Confidence_0.700_Length_651 Locus_11613_Transcript_21/21_Confidence_0.375_Length_1689 Locus 11613 Transcript 21/21 Confidence 0.375 Length 1689 Locus_11613_Transcript_21/21_Confidence_0.375_Length_1689 Locus_11613_Transcript_21/21_Confidence_0.375_Length_1689 Locus 11774 Transcript 16/21 Confidence 0.636 Length 4369 Locus_11774_Transcript_16/21_Confidence_0.636_Length_4369 Locus_11778_Transcript_4/5_Confidence_0.667_Length_847 Locus_11778_Transcript_4/5_Confidence_0.667_Length_847

\begin{tabular}{|c|c|c|c|c|c|c|c|}
\hline REVERSE & 55.47 & 42.86 & TCATGGCAAGTAGTCAGAAAC & & & & \\
\hline FORWARD & 55.07 & 38.1 & TGAGGATGATGATCTTAATGG & 151 & GAT & GATGATGATGATGATGAT & 18 \\
\hline REVERSE & 54.47 & 33.33 & ACAATCCTAAATGCTTTGCTA & & & & \\
\hline FORWARD & 55.6 & 42.86 & GTAGAGCTTCCAAAGCTGTTT & 175 & CTT & СТTCTTCTTCTTCTTCTTCTT & 21 \\
\hline REVERSE & 55.31 & 47.62 & $\begin{array}{l}\text { CTCAGAGCCTTTTACTTAGCC } \\
\end{array}$ & & & & \\
\hline FORWARD & 55.73 & 42.86 & AAGAAGTAAGGGGTGTTGCTA & 159 & GTG & GTGGTGGTGGTGGTGGTG & 18 \\
\hline REVERSE & 55.62 & 33.33 & ATCCAAAAGAAAGCTCATGTT & & & & \\
\hline FORWARD & 54.44 & 38.1 & TTGATATTACCAGGAATCTGC & 164 & $\mathrm{AGC}$ & AGCAGCAGCAGCAGCAGC & 18 \\
\hline REVERSE & 54.11 & 47.62 & AGTAATCCTCTCCTCCTTCAC & & & & \\
\hline FORWARD & 55.11 & 22.73 & AAAATGTTGTGCAATTTTGTAA & 143 & ATA & ATAATAATAATAATAATA & 18 \\
\hline REVERSE & 55.16 & 36.36 & TGAGATTGAAGAAGACTGTGAA & & & & \\
\hline FORWARD & 54.75 & 42.86 & AGAGAAAGGACTGATCGAAGT & 148 & CTT & CTTCTTCTTCTTCTTCTT & 18 \\
\hline REVERSE & 55.12 & 42.86 & GCAGCTTCAATGTCTGTTATC & & & & \\
\hline FORWARD & 54.6 & 38.1 & ATCATAACCGAAAAGAGAGGT & 163 & GGT & GGTGGTGGTGGTGGTGGT & 18 \\
\hline REVERSE & 54.79 & 45 & AACAGATCTGGCACGTAGAT & & & & \\
\hline FORWARD & 54.97 & 42.86 & CTTACAGATCAGCAATCCAAC & 147 & $\mathrm{AGA}$ & AGAAGAAGAAGAAGAAGAAGA & 21 \\
\hline REVERSE & 54.78 & 33.33 & TTTACCTCCAATTATGTTCCA & & & & \\
\hline FORWARD & 55.42 & 38.1 & AGAATCTGGCTGGAACATAAT & 149 & $\mathrm{AGA}$ & AGAAGAAGAAGAAGAAGAAGAAGA & 24 \\
\hline REVERSE & 54.2 & 42.86 & ACAAACACTTTGGTGTAGGTC & & & & \\
\hline FORWARD & 54.55 & 42.86 & CTAAAAGAACTGCTTCTGGTG & 149 & TTA & TTATTATTATTATTATTA & 18 \\
\hline REVERSE & 55.29 & 47.62 & AGGCACTCAAACCTCTCTTAC & & & & \\
\hline FORWARD & 53.8 & 38.1 & TTTGTGTTGAGATCTGAAGTG & 170 & $\mathrm{CGG}$ & CGGCGGCGGCGGCGGCGG & 18 \\
\hline REVERSE & 55.48 & 52.38 & AGTCTCTACGCTTACGACTCC & & & & \\
\hline FORWARD & 54.57 & 33.33 & TCACACAATTCAATTTCACAG & 144 & $\mathrm{CAA}$ & CAACAACAACAACAACAACAACAA & 24 \\
\hline REVERSE & 55.43 & 38.1 & CATCGTAGATTGCAAGTGATT & & & & \\
\hline FORWARD & 55.32 & 35 & ATCCCATCTTTCCСТAAAAA & 150 & CCT & ССТССТССТССТССТССТССТ & 21 \\
\hline REVERSE & 55.05 & 42.86 & TTGCAGAGAGAAAAGAGTCTG & & & & \\
\hline FORWARD & 54.07 & 42.86 & TTTTCTCTCTGCAACTCTCTC & 155 & CCT & ССТССТССТССТССТССТ & 18 \\
\hline REVERSE & 55.09 & 42.86 & AAGAGTCTGTTTTGGGAGAAC & & & & \\
\hline FORWARD & 55.12 & 35 & AAAATGCAGATCCAATCAAC & 132 & CCT & ССТССТССТССТССТССТССТ & 21 \\
\hline REVERSE & 55.05 & 42.86 & TTGCAGAGAGAAAAGAGTCTG & & & & \\
\hline FORWARD & 59.09 & 50 & AAGATGGACGGAGATGGAAG & 170 & GAG & GAGGAGGAGGAGGAGGAG & 18 \\
\hline REVERSE & 56.19 & 38.1 & AAAATGGAGCTCATTCTGAAG & & & & \\
\hline FORWARD & 55.47 & 38.1 & AGTTTAACCGAAACGAAAGAG & 142 & TCC & ТССТССТССТССТССТСС & 18 \\
\hline REVERSE & 54.72 & 42.86 & GACGGAAGAAGATTAGGTTTC & & & & \\
\hline FORWARD & 54.72 & 42.86 & GTCGGAAACCTAATCTTCTTC & 152 & TCC & ТССТССТССТССТССТСС & 18 \\
\hline REVERSE & 54.81 & 47.62 & СТTCTCCATCTCTCTTTCCTC & & & & \\
\hline FORWARD & 54.57 & 38.1 & TCAGTAGAAGTTGCACAAACA & 136 & $\mathrm{AAC}$ & AACAACAACAACAACAAC & 18 \\
\hline REVERSE & 54.89 & 33.33 & ATGTATGGGCTTTTGTTGTTA & & & & \\
\hline FORWARD & 55.08 & 38.1 & AACATTCTGTAACAGCAGCAT & 146 & TTG & TTGTTGTTGTTGTTGTTG & 18 \\
\hline REVERSE & 54.42 & 33.33 & GCAATTTTATCACAGCTTTTC & & & & \\
\hline
\end{tabular}


Locus_11819_Transcript_6/9_Confidence_0.545_Length_1400 Locus_11819_Transcript_6/9 Confidence 0.545 Length 1400 Locus_11840_Transcript_8/11_Confidence_0.647_Length_1299 Locus 11840 Transcript 8/11 Confidence 0.647 Length_1299 Locus_11877_Transcript 77/9_Confidence_0.583_Length_1045 Locus_11877_Transcript_7/9_Confidence_0.583_Length_1045 Locus_11877_Transcript_7/9_Confidence_0.583_Length_1045 Locus 11877 Transcript $7 / 9$ Confidence 0.583 Length 1045 Locus_12123_Transcript_6/7_Confidence_0.647_Length_621 Locus 12123 Transcript 6/7 Confidence 0.647 Length 621 Locus_12157_Transcript_2/3_Confidence_0.769_Length_339 Locus_12157_Transcript_2/3_Confidence_0.769_Length_339 Locus_12277_Transcript_9/12_Confidence_0.664_Length_1200 Locus_12277_Transcript_9/12_Confidence_0.664_Length_1200 Locus_12287_Transcript_1/6_Confidence_0.636_Length_705 Locus_12287_Transcript_1/6_Confidence_0.636_Length_705 Locus_12287_Transcript_1/6_Confidence_0.636_Length_705 Locus_12287_Transcript_1/6 Confidence 0.636 _Length 705

Locus_12348_Transcript_27/41 Confidence 0.534_Length_1122 Locus_12348_Transcript_27/41_Confidence_0.534_Length_1122 Locus_12392 Transcript_8/14 Confidence_0.645_Length 766 Locus_12392_Transcript_8/14_Confidence_0.645_Length_766 Locus_12647_Transcript55/8_Confidence_0.711_Length_1078 Locus 12647 Transcript 5/8 Confidence 0.711 Length 1078 Locus_12647_Transcript_5/8_Confidence_0.711_Length_1078 Locus_12647_Transcript_5/8_Confidence_0.711_Length_1078 Locus 12880 Transcript 3/7 Confidence 0.778 Length 1955 Locus_12880_Transcript_3/7_Confidence_0.778_Length_1955 Locus_12919_Transcript_4/10_Confidence_0.710_Length 776 Locus 12919 Transcript 4/10 Confidence 0.710 Length 776 Locus_12982_Transcript_11/19_Confidence_0.585_Length_1877 Locus 12982 Transcript $11 / 19$ Confidence 0.585 Length 1877 Locus_12995_Transcript_5/5_Confidence_0.500_Length_443 Locus_12995_Transcript_5/5_Confidence 0 0.500_Length 443 Locus 13273 Transcript $8 / 10$ Confidence_0.644 Length 758 Locus_13273_Transcript_8/10_Confidence_0.644_Length_758 Locus_13395_Transcript_1/2_Confidence_0.750_Length_388 Locus_13395_Transcript_1/2_Confidence_0.750_Length_388 Locus_13476_Transcript_6/8_Confidence_0.630_Length_974 Locus_13476_Transcript_6/8_Confidence_0.630_Length_974 Locus_13690_Transcript_4/6_Confidence_0.762_Length_427
FORWARD REVERSE FORWARD REVERSE FORWARD REVERSE FORWARD REVERSE FORWARD REVERSE FORWARD REVERSE FORWARD REVERSE FORWARD REVERSE FORWARD REVERSE FORWARD REVERSE FORWARD REVERSE FORWARD REVERSE FORWARD REVERSE FORWARD REVERSE FORWARD REVERSE FORWARD REVERSE FORWARD REVERSE FORWARD REVERSE FORWARD REVERSE FORWARD REVERSE FORWARD
42.86 AAGGAGAAGAGCAATTGAGAG 42.86 GCAACATTCTATGGAGTCAAC

\begin{tabular}{r|r|r}
54.79 & 42.86 & DCAACATCTAGTGGGTTGA \\
\hline 58.03 & 45 & AAGTTGGTGATGGTTA
\end{tabular} $55.54 \quad 40$ TCTGCGGAAAACTATCAAAC

54.91 42.86 CGGCTAAAGAGTGAAGATACA

\begin{tabular}{r|r|r|}
55.18 & 38.1 & CTTGTTTTTCTCAATGCAGTC \\
\hline 55.36 & 33.33 & IAAAGCA
\end{tabular} $55.36 \quad 33.33$ TAAAAGCCTCAAGTGTTTCAA

\begin{tabular}{l}
55.15 \\
\hline
\end{tabular}

$54.28 \quad 28.57$ AAAAGGAATGAATAACAAGCA 42.86 ACATGCTCTGGTCTTTCTACA 28.57 TTTCAAATTAACCATTCCTCA 42.86 AATAGATGTTCTCGACTCTCG 42.86 AAGAGGATGAGGAGAATGATG 42.86 CTAATCCGACCCTAATCCTTA 38.1 GCTTTCTCACTTTCAGAATCA 45 GAAGCTCAATCAATGGAGAG 38.1 CTTTGTTTCAGCATGATACCT 38.1 GAAGAGAGGGATTTCTCAAAA 45 GAAAGGAAGATTGAGGGAAG

42.86 ATCTTTGCCACTTCGATCTAC 45 GTGGAATTTCAGTTGCTCAC

42.86 AAATACTCATCCACCTCACCT 42.86 GTCTCAGATGCGGATTAAGTA 50 GAGACAGACGGTTCTCGAAT

42.86 GTCTCAGATGCGGATTAAGTA 45 CATAACTCCCTTTCCCTTCT

42.86 TTCCTCCTCTTCTTCTTCAAC 55 CACTCGTCCACTCTGCTAAC 36.36 TCTTCTTCTCCATACAAACACA 47.62 GGAGAAGATGGAGAGAACATC 42.86 GCTATACGTTGAAAGAGACGA 38.1 TTACTTTGCTGTATTGGATGG

40 ACCTTCAAATCCCAATTCAC 38.1 TAGGAGTGATTGGAAAACTCA 33.33 ACCAATCTCATAAACAAGCAA 52.38 AAGTAGGAGAGGAGAGTCGAG 42.86 GTGGGTTAGGTGAACGATTAT 50 GAGATCAATGGAGTCTCTCG

42.86 ATCTGCTTTACATGAGAGTCG 38.1 GCAACAATTTCATATGGAGAG 38.1 TCTTGCTTTTCTTCTATGGAG

\begin{tabular}{|c|c|c|c|}
\hline \multicolumn{2}{|c|}{\begin{tabular}{l|l|l}
196 & $\mathrm{AGG}$ \\
\end{tabular}} & AGGAGGAGGAGGAGGAGG & 18 \\
\hline 163 & GAG & GAGGAGGAGGAGGAGGAGGAG & 21 \\
\hline 15( & CTT & СTTCTTCTTCTTCTTCTT & 18 \\
\hline & & & \\
\hline 158 & AAG & AAGAAGAAGAAGAAGAAGAAG & 21 \\
\hline 14. & GAA & GAAGAAGAAGAAGAAGAAGAA & 21 \\
\hline$\overline{176}$ & $\mathrm{AGA}$ & AGAAGAAGAAGAAGAAGAAGAAGA & 24 \\
\hline & & & \\
\hline 157 & $\mathrm{CCG}$ & CCGCCGCCGCCGCCGCCGCCG & 21 \\
\hline $152,{ }^{2}$ & CTT & CTTCTTCTTCTTCTTCTT & 18 \\
\hline & & & \\
\hline 146 & CTG & CTGCTGCTGCTGCTGCTG & 18 \\
\hline & & & \\
\hline 196 & CGG & CGGCGGCGGCGGCGGCGGCGG & 21 \\
\hline 136 & GAA & GAAGAAGAAGAAGAAGAAGAAGAAGAA & 30 \\
\hline & & & \\
\hline 14. & CGG & CGGCGGCGGCGGCGGCGG & 18 \\
\hline $108+20$ & ICFC & 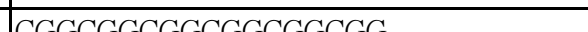 & \\
\hline & & & \\
\hline 202 & GGA & GGAGGAGGAGGAGGAGGAGGAGGA & 24 \\
\hline & & & \\
\hline 122 & CCT & ССТССТССТССТССТССТССТ & 21 \\
\hline & & & \\
\hline 16 & AGA & AGAAGAAGAAGAAGAAGAAGAAGA & 24 \\
\hline $15 \mathrm{c}$ & CTC & СТССТССТССТССТССТССТС & 21 \\
\hline & & & \\
\hline 13 & CCT & ССТССТССТССТССТССТ & 18 \\
\hline 137 & $\overline{\text { GGA }}$ & GGAGGAGGAGGAGGAGGA & 18 \\
\hline & & & \\
\hline 15 & GAA & GAAGAAGAAGAAGAAGAA & 18 \\
\hline & & & \\
\hline 140 & CTG & CTGCTGCTGCTGCTGCTGCTGCTGCTG & 27 \\
\hline
\end{tabular}


Locus_13690_Transcript_4/6_Confidence_0.762_Length_427 Locus_13809_Transcript_1/1_Confidence_0.000 Length 250 Locus_13809_Transcript_1/1_Confidence_0.000_Length_250 Locus_14274_Transcript_3/3_Confidence_0.956_Length_634 Locus_14274_Transcript_3/3_Confidence_0.956_Length_634 Locus_14304_Transcript_5/6_Confidence_0.882_Length_879 Locus 14304 Transcript $5 / 6$ Confidence 0.882 Length 879 Locus_14344_Transcript_ $1 / 2$ Confidence 0.884 _Length_ 352 Locus_14344_Transcript_1/2_Confidence_0.884_Length_352 Locus 14525 Transcript $1 / 1$ Confidence 0.970 Length 331 Locus_14525_Transcript_1/1_Confidence_0.970_Length_331 Locus_14759_Transcript_1/2_Confidence_0.980_Length_409 Locus_14759_Transcript_1/2_Confidence_0.980_Length_409 Locus_14803_Transcript_2/3_Confidence_0.929_Length_1021 Locus_14803_Transcript_2/3_Confidence_0.929_Length_1021 Locus_14803_Transcript_2/3_Confidence_0.929_Length_1021 Locus_14803_Transcript_2/3_Confidence_0.929_Length_102 Locus_14952_Transcript_1/1_Confidence_1.000_Length_733 Locus 14952 Transcript $1 / 1$ Confidence 1.000 Length 733 Locus_15000_Transcript_1/1_Confidence_1.000_Length_271 Locus 15000 Transcript 1/1 Confidence 1.000 Length 271 Locus_15001_Transcript_1/1_Confidence_1.000_Length_277 Locus 15001 Transcript $1 / 1$ Confidence 1.000 Length 277 Locus 15336 Transcript 1/1 Confidence 1.000 Length 372 Locus_15336_Transcript_1/1_Confidence_1.000_Length_372 Locus_15337_Transcript_1/1_Confidence_1.000_Length_525 Locus 15337 Transcript 1/1 Confidence 1.000 Length 525 Locus_15339_Transcript_1/1_Confidence_1.000_Length_781 Locus 15339 Transcript 1/1 Confidence 1.000 Length 781 Locus_15339_Transcript_1/1_Confidence_1.000_Length_781 Locus_15339_Transcript_1/1_Confidence_1.000_Length_781 Locus_15340_Transcript_1/1_Confidence_1.000_Length_329 Locus_15340_Transcript_1/1_Confidence_1.000_Length_329 Locus_15344_Transcript_1/1_Confidence_1.000_Length_482 Locus 15344 Transcript 1/1 Confidence 1.000 Length 482 Locus_15975_Transcript_1/1_Confidence_1.000_Length_541 Locus_15975_Transcript $1 / 1$ Confidence 1.000 Length 541 Locus_15976_Transcript_1/1_Confidence_1.000_Length_542 Locus_15976_Transcript_1/1_Confidence_1.000_Length_542 Locus_15977_Transcript_1/1_Confidence_1.000_Length_441 Locus_15977_Transcript_1/1_Confidence_1.000_Length_441

\begin{tabular}{|c|c|c|c|c|c|c|c|}
\hline REVERSE & 55.05 & 42.86 & TCCATCACTTTCACATACCTC & & & & \\
\hline FORWARD & 55.91 & 42.86 & ATTTCCTTACGACATGAGAGG & 151 & GAA & GAAGAAGAAGAAGAAGAA & 18 \\
\hline REVERSE & 55.03 & 42.86 & AGTGGCTGGACCTTATATTTC & & & & \\
\hline FORWARD & 54.44 & 38.1 & CGTAATTTAGGGTTTCCTTCT & 149 & TCT & TCTTCTTCTTCTTCTTCT & 18 \\
\hline REVERSE & 55.16 & 28.57 & ATCAAACGCATCAAAACTAAA & & & & \\
\hline FORWARD & 55.04 & 33.33 & GATTTGCTTGAAACAGCTTTA & 158 & ATC & ATCATCATCATCATCATC & 18 \\
\hline REVERSE & 54.94 & 33.33 & AGGTTGAAAGGAAGAAGAAAA & & & & \\
\hline FORWARD & 55.2 & 42.86 & TTACTACGCTCAACCCATAGA & 207 & ATT & ATTATTATTATTATTATT & 18 \\
\hline REVERSE & 54.96 & 42.86 & GGACACATCTCCATCAGAATA & & & & \\
\hline FORWARD & 57.1 & 50 & AGTGTTCAAGAAGGCAGGAG & 130 & GGT & GGTGGTGGTGGTGGTGGTGGT & 21 \\
\hline REVERSE & 55.08 & 38.1 & CCACTTTAACAAATCTCAACG & & & & \\
\hline FORWARD & 55.17 & 35 & AAGAAACCAGTTGCAGAAAA & 150 & AGA & AGAAGAAGAAGAAGAAGA & 18 \\
\hline REVERSE & 54.3 & 43.48 & AGATGTAGATCTTGTACGTCTCC & & & & \\
\hline FORWARD & 55.16 & 28.57 & ATCAAACGCATCAAAACTAAA & 149 & AGA & AGAAGAAGAAGAAGAAGA & 18 \\
\hline REVERSE & 54.44 & 38.1 & CGTAATTTAGGGTTTCCTTCT & & & & \\
\hline FORWARD & 54.77 & 33.33 & ACGCATCAAAACTAAATCAAG & 151 & AGA & AGAAGAAGAAGAAGAAGAAGAAGA & 24 \\
\hline REVERSE & 54.2 & 38.1 & TCGTTTAACAGCTTCTTCTTC & & & & \\
\hline FORWARD & 54.17 & 38.1 & CCTTCTCATTCTCAAATATCG & 151 & CTC & СТССТССТССТССТССТ & 18 \\
\hline REVERSE & 55.3 & 33.33 & TTCCCTTTCTTTTGAATTAGG & & & & \\
\hline FORWARD & 54.83 & 42.86 & CAAAAGTCTGATCTGAACACC & 167 & TCT & TCTTCTTCTTCTTCTTCTTCTTCT & 24 \\
\hline REVERSE & 54.37 & 38.1 & TCTAATATACACACGGTGCAA & & & & \\
\hline FORWARD & 55.26 & 38.1 & TTCAAAAGTCTGATGAACACC & 169 & TCT & TCTTCTTCTTCTTCTTCTTCTTCT & 24 \\
\hline REVERSE & 54.37 & 38.1 & TCTAATATACACACGGTGCAA & & & & \\
\hline FORWARD & 55.31 & 42.86 & ATTTCTGGAAGCTATGGAGAG & 127 & AGA & AGAAGAAGAAGAAGAAGAAGAAGA & 24 \\
\hline REVERSE & 55.03 & 38.1 & TTTAGGGTTTCTTCACAGACA & & & & \\
\hline FORWARD & 55.16 & 28.57 & AACGCATCAAAACTAAATCAA & 150 & AGA & AGAAGAAGAAGAAGAAGAAGAAGA & 24 \\
\hline REVERSE & 54.08 & 38.1 & CATCGTTTAACAGCTTCTTCT & & & & \\
\hline FORWARD & 55.16 & 28.57 & ATCAAACGCATCAAAACTAAA & 149 & AGA & AGAAGAAGAAGAAGAAGA & 18 \\
\hline REVERSE & 54.44 & 38.1 & CGTAATTTAGGGTTTCCTTCT & & & & \\
\hline FORWARD & 54.77 & 33.33 & ACGCATCAAAACTAAATCAAG & 151 & AGA & AGAAGAAGAAGAAGAAGAAGAAGA & 24 \\
\hline REVERSE & 54.2 & 38.1 & TCGTTTAACAGCTTCTTCTTC & & & & \\
\hline FORWARD & 55.72 & 47.62 & ACTCTCCATCTCATCTTCACC & 154 & $\mathrm{AAG}$ & AAGAAGAAGAAGAAGAAGAAG & 21 \\
\hline REVERSE & 55.25 & 47.62 & GGCGGAGATAGTGTTCTTACT & & & & \\
\hline FORWARD & 54.94 & 36.36 & AGCAAACAAGAAGAAGAAGAAG & 148 & $\mathrm{AAG}$ & AAGAAGAAGAAGAAGAAGAAGAAG & 24 \\
\hline REVERSE & 54.55 & 38.1 & TGTTCACATACACGCTTTCTA & & & & \\
\hline FORWARD & 56.19 & 34.78 & TGTTCTGAGAGGTTTGTATTTTG & 162 & $\mathrm{AAG}$ & \begin{tabular}{|l} 
AAGAAGAAGAAGAAGAAG \\
\end{tabular} & 18 \\
\hline REVERSE & 59.06 & 55 & GGATCTCACCGGATCTCACT & & & & \\
\hline FORWARD & 56.19 & 34.78 & TGTTCTGAGAGGTTTGTATTTTG & 162 & $\mathrm{AAG}$ & AAGAAGAAGAAGAAGAAG & 18 \\
\hline REVERSE & 59.06 & 55 & GGATCTCACCGGATCTCACT & & & & \\
\hline FORWARD & 56.19 & 34.78 & TGTTCTGAGAGGTTTGTATTTTG & 162 & $\mathrm{AAG}$ & AAGAAGAAGAAGAAGAAG & 18 \\
\hline REVERSE & 59.06 & & GGATCTCACCGGATCTCACT & & & & \\
\hline
\end{tabular}


Locus_15978_Transcript_1/1_Confidence_1.000_Length_460 Locus 15978 Transcript 1/1 Confidence 1.000 Length 460 Locus_16041_Transcript_1/1_Confidence_1.000_Length_562 Locus_16041_Transcript_1/1_Confidence_1.000_Length_562 Locus 16042_Transcript 1/1 Confidence 1.000 Length 413 Locus_16042_Transcript_1/1_Confidence_1.000_Length_413 Locus_16514_Transcript_1/1_Confidence_1.000_Length_259 Locus 16514 Transcript 1/1 Confidence 1.000 Length 259 Locus_17142_Transcript_1/1_Confidence_1.000_Length_457 Locus 17142 Transcript $1 / 1$ Confidence 1.000 Length 457 Locus_17450_Transcript_1/1_Confidence_1.000_Length_700 Locus_17450_Transcript_1/1_Confidence 1.000 Length_700 Locus_17489_Transcript_1/1_Confidence_1.000_Length_483 Locus_17489_Transcript_1/1_Confidence_1.000_Length_483 Locus_17492_Transcript_1/1_Confidence_1.000_Length_501 Locus 17492 Transcript 1/1 Confidence 1.000 Length 501 Locus_17493_Transcript_1/1_Confidence_1.000_Length_579 Locus 17493 Transcript 1/1 Confidence 1.000 Length 579 Locus_17797_Transcript_1/1_Confidence_1.000_Length_244 Locus_17797_Transcript_1/1_Confidence_1.000_Length_244 Locus_17810_Transcript_1/1_Confidence_1.000_Length_335 Locus_17810_Transcript_1/1_Confidence_1.000_Length_335 Locus_17811_Transcript_1/1_Confidence_1.000_Length_311 Locus 17811 Transcript $1 / 1$ Confidence 1.000 Length 311 Locus_17812_Transcript_1/1_Confidence_1.000_Length_315 Locus 17812 Transcript 1/1 Confidence 1.000 Length 315 Locus 18813 Transcript 1/1 Confidence 1.000 Length 519 Locus_18813_Transcript_1/1_Confidence_1.000_Length_519 Locus 18814 Transcript 1/1 Confidence 1.000 Length 521 Locus_18814_Transcript_1/1_Confidence_1.000_Length_521 Locus_18815_Transcript_1/1_Confidence_1.000_Length_342 Locus 18815 Transcript_1/1_Confidence 1.000 Length_342 Locus_19055_Transcript_1/1_Confidence_1.000_Length_235 Locus_19055_Transcript_1/1_Confidence_1.000_Length_235 Locus 19213 Transcript 1/1 Confidence 1.000 Length 656 Locus_19213_Transcript_1/1_Confidence_1.000_Length_656 Locus_19912_Transcript_1/1_Confidence_1.000_Length_831 Locus_19912_Transcript_1/1_Confidence_1.000_Length_831 Locus_20453_Transcript_1/1_Confidence_1.000_Length_405 Locus_20453_Transcript_1/1_Confidence_1.000_Length_405 Locus_21135_Transcript_1/1_Confidence_1.000_Length_325

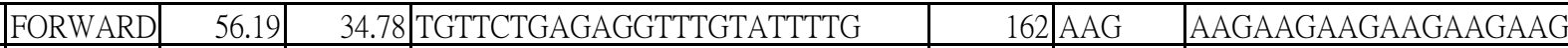
\begin{tabular}{|l|r|r|}
\hline REVERSE & 59.06 & 55 GGATCTCACCGGATCTCACT \\
\hline
\end{tabular}

FORWARD REVERSE

FORWARD REVERSE FORWARD REVERSE FORWARD REVERSE

FORWARD REVERSE FORWARD REVERSE FORWARD REVERSE FORWARD REVERSE FORWARD REVERSE FORWARD REVERSE FORWARD REVERSE FORWARD REVERSE FORWARD REVERSE FORWARD REVERSE FORWARD REVERSE FORWARD REVERSE

FORWARD REVERSE FORWARD REVERSE FORWARD REVERSE FORWARD

54.93 42.86 ATCTTGTACGTCTCCGTTGTA $55.17 \quad 40$ GAAACCAGTTGCAGAAAAAG

55.57 47.62 AGATCTTGTACGTCTCCGTCT

55.17 35 AAAAGAAACCAGTTGCAGAA 55.42 42.86 AGGAAGAGTTCGAAGTTGAAG

47.62 TTAAGCTCTCCTCACTCCTCT

38.1 ACTCTTTTGGCTTCTATTTGG

33.33 GAAGAAAAGAAGAAGAAGAAGAAG

42.86 CTTAGTGGGATAAAGGCTTGT

38.1 ATCACGAAGTATGCAAATAGC

38.1 CGTAATTTAGGGTTTCCTTCT

28.57 ATCAAACGCATCAAAACTAAA

33.33 ATGATAAAGGCGAAGAAGATT

38.1 GTTTCCATTTCTGGAAGCTAT

33.33 ATGATAAAGGCGAAGAAGATT

38.1 GTTTCCATTTCTGGAAGCTAT

38.1 CGCTCTTCTTCTTTCTTCTTT

42.86 CAAGGGTTAACAATCTGTACG

38.1 TCCTCTCTCTTTTTGTCCTTT

40 TAAACTCTCGTTCGCTTTGT

38.1 TCCTCTCTCTTTTTGTCCTTT

40 TAAACTCTCGTTCGCTTTGT

38.1 TCCTCTCTCTTTTTGTCCTTT

40 TAAACTCTCGTTCGCTTTGT

47.62 GATCTTGTAAGTCTCGGTTCC 45 GTGGAGGAGAAGGAAAAGAA

47.62 GATCTTGTAAGTCTCGGTTCC

\begin{tabular}{r|r|r}
56 & 45 & GTGGAGGAGAAGGAAAAGAA \\
54.92 & 42.86 & TCAGGACCTTGAAGATGTAGA \\
\hline
\end{tabular} 47.62 GAGGAGAAGGAAAAGAAGACC

42.86 GAGAATGATGGAGAAGAGGAT

42.86 CCTTATCGACCCTAATCCTTA

42.86 GAAGGAGAAGAAAGAAGGAGA

38.1 CTTGATCAAACAGAACAGCAT

33.33 TCACTTTTTGATTGACACACA

33.33 AGAAAATTGAAGAATCCAAGG

\begin{tabular}{|l|l|l|}
57.12 & 47.62 & GTGGAGGAGAAGGAAAAGAAG \\
\hline
\end{tabular}

$58.52 \quad 50$ CTTCTCAGCCTTGGGTTTCT

55.49 50|AAGAAGAAGCAGAGGAGGAG

\begin{tabular}{|c|c|c|c|}
\hline 162 & $\mathrm{AAG}$ & AAGAAGAAGAAGAAGAAG & 18 \\
\hline 155 & CTT & CTTCTTCTTCTTCTTCTT & 18 \\
\hline 146 & CTT & CTTCTTCTTCTTCTTCTT & 18 \\
\hline & & & \\
\hline 104 & GAA & GAAGAAGAAGAAGAAGAAGAA & 21 \\
\hline 150 & CTC & СТССТССТССТССТССТС & 18 \\
\hline 157 & GTT & GTTGTTGTTGTTGTTGTT & 18 \\
\hline & & & \\
\hline 149 & TCT & TCTTCTTCTTCTTCTTCT & 18 \\
\hline 149 & TCT & TCTTCTTCTTCTTCTTCT & 18 \\
\hline & & & \\
\hline 149 & TCT & TCTTCTTCTTCTTCTTCT & 18 \\
\hline 157 & TTC & TTCTTCTTCTTCTTCTTCTTC & 21 \\
\hline 174 & TCT & TCTTCTTCTTCTTCTTCTTCTTCT & 24 \\
\hline 174 & TCT & TCTTCTTCTTCTTCTTCTTCTTCT & 24 \\
\hline & & & \\
\hline 178 & TCT & TCTTCTTCTTCTTCTTCTTCT & 21 \\
\hline 150 & TCT & TCTTCTTCTTCTTCTTCT & 18 \\
\hline 150 & TCT & TCTTCTTCTTCTTCTTCT & 18 \\
\hline & & 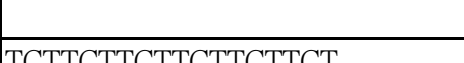 & \\
\hline 166 & TCT & TCTTCTTCTTCTTCTTCT & 18 \\
\hline 148 & $\mathrm{CCG}$ & CCGCCGCCGCCGCCGCCGCCG & 21 \\
\hline 152 & AGA & AGAAGAAGAAGAAGAAGA & 18 \\
\hline 147 & CTT & |СТTCTTCTTCTTCTTCTT & 18 \\
\hline & & & \\
\hline 144 & AAG & AAGAAGAAGAAGAAGAAG & 18 \\
\hline 160 & GAG & GAGGAGGAGGAGGAGGAG & 18 \\
\hline
\end{tabular}




\begin{tabular}{|c|c|c|c|c|c|c|c|c|}
\hline Locus_21135_Transcript_1/1_Confidence_1.000_Length_325 & REVERSE & 56.44 & 45 & ATAACCTCCTCCCAACAATG & & & & \\
\hline Locus_21136_Transcript_1/1_Confidence_1.000_Length_280 & FORWARD & 55.49 & 50 & AAGAAGAAGCAGAGGAGGAG & 103 & GAG & GAGGAGGAGGAGGAGGAG & 18 \\
\hline Locus_21136_Transcript_1/1_Confidence_1.000_Length_280 & REVERSE & 56.17 & 38.1 & AACTCGACGACAAAAACTTTC & & & & \\
\hline Locus_21137_Transcript_1/1_Confidence_1.000_Length_223 & FORWARD & 55.49 & 50 & AAGAAGAAGCAGAGGAGGAG & 198 & GAG & GAGGAGGAGGAGGAGGAG & 18 \\
\hline Locus_21137_Transcript_1/1_Confidence_1.000_Length_223 & REVERSE & 54.55 & 38.1 & CAAAACCAGAGATGGAAGTAA & & & & \\
\hline Locus_21353_Transcript_1/1_Confidence_1.000_Length_278 & FORWARD & 57.58 & 45 & CСACТCCCCACACTTAAAAA & 159 & $\mathrm{CCT}$ & ССТССТССТССТССТССТССТ & 21 \\
\hline Locus_21353_Transcript_1/1_Confidence_1.000_Length_278 & REVERSE & 55.18 & 45 & AGTGAGAATCGTCGAACATC & & & & \\
\hline Locus_21436_Transcript_1/1_Confidence_1.000_Length_483 & FORWARD & 54.08 & 52.38 & GAGGAGGAGAGAGAGAGAAAG & 132 & AGA & AGAAGAAGAAGAAGAAGA & 18 \\
\hline Locus_21436_Transcript_1/1_Confidence_1.000_Length_483 & REVERSE & 54.31 & 42.86 & CTGTTCTTCCTGTTTCTGAAG & & & & \\
\hline Locus_21437_Transcript_1/1_Confidence_1.000_Length_538 & FORWARD & 54.08 & 52.38 & GAGGAGGAGAGAGAGAGAAAG & 132 & $\mathrm{AGA}$ & AGAAGAAGAAGAAGAAGA & 18 \\
\hline Locus_21437_Transcript_1/1_Confidence_1.000_Length_538 & REVERSE & 54.31 & 42.86 & CTGTTCTTCCTGTTTCTGAAG & & & & \\
\hline Locus_21438_Transcript_1/1_Confidence_1.000_Length_479 & FORWARD & 54.38 & 47.62 & AGAAAGAGGGAGAGAGAGATG & 165 & $\mathrm{AGA}$ & AGAAGAAGAAGAAGAAGA & 18 \\
\hline Locus_21438_Transcript_1/1_Confidence_1.000_Length_479 & REVERSE & 54.74 & 38.1 & TGTTCTTCCTGTTTTCTGAAG & & & & \\
\hline Locus_21439_Transcript_1/1_Confidence_1.000_Length_534 & FORWARD & 54.38 & 47.62 & AGAAAGAGGGAGAGAGAGATG & 165 & $\mathrm{AGA}$ & AGAAGAAGAAGAAGAAGA & 18 \\
\hline Locus_21439_Transcript_1/1_Confidence_1.000_Length_534 & REVERSE & 54.74 & 38.1 & TGTTCTTCCTGTTTTCTGAAG & & & & \\
\hline Locus_21440_Transcript_1/1_Confidence_1.000_Length_558 & FORWARD & 54.08 & 52.38 & GAGGAGGAGAGAGAGAGAAAG & 132 & AGA & AGAAGAAGAAGAAGAAGA & 18 \\
\hline Locus_21440_Transcript_1/1_Confidence_1.000_Length_558 & REVERSE & 54.31 & 42.86 & CTGTTCTTCCTGTTTCTGAAG & & & & \\
\hline Locus_21441_Transcript_1/1_Confidence_1.000_Length_562 & FORWARD & 54.08 & 52.38 & GAGGAGGAGAGAGAGAGAAAG & 132 & $\mathrm{AGA}$ & AGAAGAAGAAGAAGAAGA & 18 \\
\hline Locus_21441_Transcript_1/1_Confidence_1.000_Length_562 & REVERSE & 54.31 & 42.86 & CTGTTCTTCCTGTTTCTGAAG & & & & \\
\hline Locus_21858_Transcript_1/1_Confidence_1.000_Length_316 & FORWARD & 55.72 & 47.62 & ACTCTCCATCTCATCTTCACC & 135 & $\mathrm{AAG}$ & AAGAAGAAGAAGAAGAAGAAGAAG & 24 \\
\hline Locus_21858_Transcript_1/1_Confidence_1.000_Length_316 & REVERSE & 55.25 & 47.62 & GGCGGAGATAGTGTTCTTACT & & & & \\
\hline Locus_21894_Transcript_1/1_Confidence_1.000_Length_394 & FORWARD & 54.92 & 45.45 & GAGAAATAGACGAGCTCATACC & 185 & GAC & GACGACGACGACGACGACGACGACGAC & 27 \\
\hline Locus_21894_Transcript_1/1_Confidence_1.000_Length_394 & REVERSE & 54.92 & 42.86 & ATCTCTCTTGCTTCTTGTCCT & & & & \\
\hline
\end{tabular}


(C) Tetranucleotide repeat more than 5 times.

Seq ID

Locus_361_Transcript_8/10_Confidence_0.667_Length_2265 Locus_361_Transcript_8/10_Confidence_0.667_Length 2265 Locus 361 Transcript 8/10 Confidence 0.667 Length 2265 Locus_361_Transcript_8/10_Confidence_0.667_Length_2265 Locus_898_Transcript_89/133_Confidence_0.333_Length_3222 Locus 898 Transcript 89/133 Confidence 0.333 Length 3222 Locus_1156_Transcript_76/96_Confidence_0.242_Length_1523 Locus_1156_Transcript_76/96_Confidence_0.242_Length_1523 Locus_1268_Transcript_4/12_Confidence_0.656_Length_1288 Locus_1268_Transcript_4/12_Confidence_0.656_Length_1288 Locus_1633_Transcript_29/35_Confidence_0.365_Length_2130 Locus_1633_Transcript_29/35_Confidence_0.365_Length_2130 Locus_2054_Transcript_31/32_Confidence_0.609_Length_1880 Locus_2054_Transcript_31/32_Confidence_0.609_Length_1880 Locus_2641_Transcript_39/55_Confidence_0.330_Length_3582 Locus 2641 Transcript 39/55 Confidence 0.330 Length 3582 Locus_3522_Transcript_36/40_Confidence_0.569_Length_2763 Locus_3522_Transcript_36/40 Confidence 0.569 Length 2763 Locus 3567 Transcript 11/41 Confidence 0.437 Length 1143 Locus_3567_Transcript_11/41_Confidence_0.437_Length_1143 Locus_3707_Transcript_29/31_Confidence_0.653_Length_4149 Locus 3707 Transcript 29/31 Confidence 0.653 Length 4149 Locus_4245_Transcript_17/22_Confidence_0.632_Length_2667 Locus_4245_Transcript_17/22_Confidence_0.632_Length_2667 Locus 4600 Transcript 27/48 Confidence 0.520 Length 1792 Locus_4600_Transcript_27/48_Confidence_0.520_Length_1792 Locus_4824_Transcript_11/26_Confidence_0.577_Length_1571 Locus 4824 Transcript 11/26 Confidence 0.577 Length 1571 Locus_4824_Transcript_11/26_Confidence_0.577_Length_1571 Locus_4824_Transcript_11/26_Confidence_0.577_Length_1571 Locus 4992 Transcript 40/52 Confidence 0.508 Length 2753 Locus 4992 Transcript 40/52 Confidence 0.508 Length 2753 Locus_5532_Transcript_5/19_Confidence_0.393_Length_2148 Locus_5532_Transcript_5/19_Confidence_0.393_Length_2148 Locus 5532 Transcript 5/19 Confidence 0.393 Length 2148 Locus_5532_Transcript_5/19_Confidence_0.393_Length_2148 Locus_5626_Transcript_20/89_Confidence_0.574_Length_2090 Locus_5626 Transcript_20/89 Confidence_0.574_Length_2090 Locus_5657_Transcript_52/60_Confidence_0.469_Length_1878 Locus 5657 Transcript 52/60 Confidence 0.469 Length_1878

\begin{tabular}{|c|c|c|c|c|c|c|c|c|}
\hline Orientation & Start & $\mathrm{tm}$ & GC\% & Seq & Prod size & Motif & SSR & SSRLen \\
\hline FORWARD & 161 & 55.25 & 33.33 & CTCAAATAACAAATCCACCAA & 165 & TTAT & TTATTTATTTATTTATTTAT & 20 \\
\hline FORWARD & 175 & 55.12 & 38.1 & GTGGTAACGCAAAAACATAAG & 156 & TTTA & TTTATTTATTTATTTATTTATTTA & 24 \\
\hline REVERSE & 330 & 54.64 & 33.33 & TCAAGCTTCAAAGTAAATGCT & & & & \\
\hline FORWARD & 134 & 54.03 & 57.14 & GAGAGGAGAGAGGAGAGAGAG & 150 & CGGT & CGGTCGGTCGGTCGGTCGGT & 20 \\
\hline REVERSE & 283 & 55.01 & 33.33 & CACCATCACTTTTATTTTTGC & & & & \\
\hline FORWARD & 119 & 54.03 & 57.14 & GGGAGAGAGAGAGAGAGAGAG & 153 & CGGT & CGGTCGGTCGGTCGGTCGGTCGGTCGGT & 28 \\
\hline REVERSE & 271 & 53.88 & 33.33 & CTGTCAACGATTTTCTCTTTT & & & & \\
\hline FORWARD & 218 & 54.67 & 42.86 & TATAGGCTTCTGAACATCTGG & 154 & TGTT & TGTTTGTTTGTTTGTTTGTT & 20 \\
\hline REVERSE & 371 & 55.17 & 33.33 & GAAAACCTTGGCATTTCTATT & & & & \\
\hline FORWARD & 172 & 55.2 & 42.86 & TCTCTGATACCAATCCCCTAT & 144 & TTAC & TTACTTACTTACTTACTTACTTACTTACTTA & 52 \\
\hline FORWARD & 131 & 54.57 & 33.33 & TGAGCTTTAAAAAGCAAAGAG & 151 & CGGT & CGGTCGGTCGGTCGGTCGGTCGGTCGGT & 28 \\
\hline REVERSE & 281 & 54.99 & 27.27 & TTGGGATAAGCTAATTTTTGTT & & & & \\
\hline FORWARD & 204 & 55.24 & 42.86 & AAGCTGTCACTTCAAGACAGA & 159 & GGAG & GGAGGGAGGGAGGGAGGGAG & 20 \\
\hline REVERSE & 362 & 55.42 & 47.62 & GTCTCCTTTGACTCCTCATTC & & & & \\
\hline FORWARD & 140 & 55.18 & 33.33 & TGTTTTCTTTGTGTGTTGTGA & 149 & TTTA & TTTATTTATTTATTTATTTA & 20 \\
\hline REVERSE & 288 & 54.92 & 38.1 & GTGCAAGATTCATGTCTTTTC & & & & \\
\hline FORWARD & 284 & 54.35 & 42.86 & GATTCATGAACTAGAGCTTGC & 134 & TATG & TATGTATGTATGTATGTATGTATGTATG & 28 \\
\hline REVERSE & 417 & 54.76 & 38.1 & CCTACGCAAATTTACATCCTA & & & & \\
\hline FORWARD & 286 & 54.35 & 42.86 & GATTCATGAACTAGAGCTTGC & 134 & TATG & TATGTATGTATGTATGTATGTATGTATG & 28 \\
\hline REVERSE & 419 & 54.76 & 38.1 & CCTACGCAAATTTACATCCTA & & & & \\
\hline FORWARD & 133 & 54.56 & 33.33 & GGAAATGATGAAGATTGCTAA & 151 & AAAT & AAATAAATAAATAAATAAAT & 20 \\
\hline REVERSE & 283 & 55.12 & 42.86 & GAGGTTAATGAAGGAGGAGAA & & & & \\
\hline
\end{tabular}




\begin{tabular}{|c|c|c|c|c|c|c|c|c|c|}
\hline Locus_5757_Transcript_17/25_Confidence_0.584_Length_810 & FORWARD & 109 & 55.18 & 23.81 & TTTGAAATTACAACGCAAAAT & 164 & AGAT & AGATAGATAGATAGATAGATAGAT & 24 \\
\hline Locus_5757_Transcript_17/25_Confidence_0.584_Length_810 & REVERSE & 272 & 55.04 & 42.86 & GGAAATGGGGAGTAATGTATC & & & & \\
\hline Locus_7547_Transcript_34/42_Confidence_0.591_Length_2987 & FORWARD & 81 & 54.91 & 28.57 & TTTGGTTTATTTCCTCAATCA & 166 & ATAA & ATAAATAAATAAATAAATAA & 20 \\
\hline Locus_7547_Transcript_34/42_Confidence_0.591_Length_2987 & REVERSE & 246 & 55.25 & 38.1 & TTTGAGAGTTTCTGCTGTTGT & & & & \\
\hline Locus_7695_Transcript_17/38_Confidence_0.556_Length_3232 & FORWARD & 276 & 54.49 & 42.86 & GTAATGCTATCTCCTGGTGAA & 146 & ATGT & ATGTATGTATGTATGTATGTATGT & 24 \\
\hline Locus_7695_Transcript_17/38_Confidence_0.556_Length_3232 & REVERSE & 421 & 54.24 & 28.57 & AAATGGTTATTTTTCATGGTG & & & & \\
\hline Locus_9903_Transcript_11/17_Confidence_0.555_Length_2770 & FORWARD & 149 & 54.19 & 57.14 & GAGCCTCTCTCTCTCTCTCTC & 145 & TTAC & TTACTTACTTACTTACTTACTTAC & 24 \\
\hline Locus_9903_Transcript_11/17_Confidence_0.555_Length_2770 & REVERSE & 293 & 54.9 & 33.33 & CCAAGTTCTGAAAGCAAATTA & & & & \\
\hline Locus_11085_Transcript_7/18_Confidence_0.575_Length_1602 & FORWARD & 140 & 54.62 & 42.86 & GTCTGCGAAGTACTCAACAAT & 144 & GAAG & $\mathrm{GAAC}$ & 20 \\
\hline Locus_11085_Transcript_7/18_Confidence_0.575_Length_1602 & REVERSE & 283 & 54.65 & 33.33 & GCTGATTTGACTAATTTGGAA & & & & \\
\hline Locus_13166_Transcript_2/5_Confidence_0.882_Length_621 & FORWARD & 273 & 55.02 & 57.14 & GACGGAGAGAGAGAGAGAGAG & 147 & CGGT & CGGTCGGTCGGTCGGTCGGTCGGT & 24 \\
\hline Locus_13166_Transcript_2/5_Confidence_0.882_Length_621 & REVERSE & 419 & 53.88 & 33.33 & CTGTCAACGATTTTCTCTTTT & & & & \\
\hline Locus_13609_Transcript_8/11_Confidence_0.610_Length_536 & FORWARD & 107 & 55.36 & 57.14 & CGCTCTCTCTCTCTCTCTCTC & 148 & TTAC & TTACTTACTTACTTACTTACTTACTTAC & 28 \\
\hline Locus_13609_Transcript_8/11_Confidence_0.610_Length_536 & REVERSE & 254 & 55 & 42.86 & AGCTAGGGTTTAGAGTTTCCA & & & & \\
\hline Locus_15225_Transcript_1/1_Confidence_1.000_Length_437 & FORWARD & 184 & 55.09 & 42.86 & TACCAATCCCCTATACCATCT & 155 & TTAC & TTACTTACTTACTTACTTACTTACTTACTTA & 32 \\
\hline Locus_15225_Transcript_1/1_Confidence_1.000_Length_437 & REVERSE & 338 & 54.68 & 33.33 & TTCATCATCTCCAACTTCATT & & & & \\
\hline Locus_15321_Transcript_1/1_Confidence_1.000_Length_524 & FORWARD & 213 & 56.43 & 45 & GATGACTTTGGGACCTGATT & 176 & GTCG & GTCGGTCGGTCGGTCGGTCG & 20 \\
\hline Locus_15321_Transcript_1/1_Confidence_1.000_Length_524 & REVERSE & 388 & 54.67 & 33.33 & TGTCAACGATTTTCTCTTTTC & & & & \\
\hline
\end{tabular}




\section{Seq ID}

Locus_17_Transcript_42/50_Confidence_0.593_Length_5741 Locus_17_Transcript_42/50_Confidence_0.593_Length_5741 Locus_17_Transcript_42/50_Confidence_0.593_Length_5741 Locus_17_Transcript_42/50_Confidence_0.593_Length_5741 Locus_164_Transcript_118/136_Confidence_0.289_Length_1757 Locus_164_Transcript_118/136_Confidence_0.289_Length_1757 Locus_337_Transcript_20/86_Confidence_0.573_Length_3129 Locus_337_Transcript_20/86_Confidence_0.573_Length_3129 Locus_391_Transcript_19/24_Confidence_0.710_Length_4275 Locus 391_Transcript_19/24_Confidence_0.710_Length_4275 Locus_391_Transcript_19/24_Confidence_0.710_Length_4275 Locus_391_Transcript_19/24_Confidence_0.710_Length_4275 Locus 505 Transcript 23/47 Confidence 0.573 Length 343 Locus 505_Transcript_23/47_Confidence_0.573 Length 3437 Locus_921_Transcript_42/65_Confidence_0.583_Length_4364 Locus_921_Transcript_42/65_Confidence_0.583_Length_4364 Locus_1256_Transcript_17/51_Confidence_0.535_Length_2636 Locus_1256_Transcript_17/51_Confidence_0.535_Length_2636 Locus_1462_Transcript_14/19_Confidence_0.587_Length_1312 Locus_1462_Transcript_14/19_Confidence_0.587_Length_1312 Locus_1949_Transcript_6/11_Confidence_0.716_Length_2361 Locus_1949_Transcript_6/11_Confidence_0.716_Length_2361 Locus_1990_Transcript_48/77_Confidence_0.570_Length_4809 Locus_1990_Transcript_48/77_Confidence_0.570_Length_4809 Locus_2143_Transcript_8/14_Confidence_0.767_Length_793 Locus_2143_Transcript_8/14_Confidence_0.767_Length_793 Locus_2623_Transcript_2/14_Confidence_0.684_Length_3447 Locus_2623_Transcript_2/14_Confidence_0.684_Length_3447 Locus_2639_Transcript_56/106_Confidence_0.388_Length_4129 Locus_2639_Transcript_56/106_Confidence_0.388_Length_4129 64 Transcript 124/133_Confidence_0.268_Length_6476 FORWARD Locus 2642_Transcript_124/133_Confidence_0.268_Length_6476 REVERSE

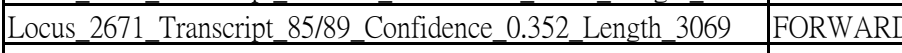
Locus_2671_Transcript_85/89_Confidence_0.352_Length_3069 Locus_2775_Transcript 11/13 Confidence 0.667 Length 3349 FORWARD

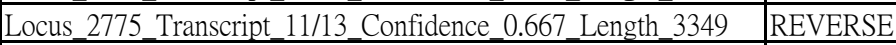
Locus_2846_Transcript_18/25_Confidence_0.629_Length_1641 FORWARD Locus 2846 Transcript 18/25 Confidence 0.629 Length 1641 REVERSE Locus 2895 Transcript 9/19 Confidence_0.656_Length_1416 Locus_2895_Transcript_9/19_Confidence_0.656_Length_1416 Locus_2920_Transcript_36/43_Confidence_0.586_Length_2898

\begin{tabular}{|c|c|c|c|}
\hline \begin{tabular}{|l|l} 
Orientation & $t$ \\
\end{tabular} & tm & $\mathrm{GC} \%$ & Seq \\
\hline FORWARD & 55.24 & 42.86 & TGTGCATAATAGGGGTGATAG \\
\hline REVERSE & 54.91 & 28.57 & TCTCAAATCCACCAAAAATTA \\
\hline FORWARD & 54.91 & 28.57 & TAATTTTTGGTGGATTTGAGA \\
\hline REVERSE & 54.28 & 33.33 & CAGCAGTAATAAACATGCAAA \\
\hline FORWARD & 55.12 & 52.38 & CCTAGACCTCCAACTCTGTCT \\
\hline REVERSE & 54.65 & 33.33 & TGCATTTCTTATTGTTTCTCC \\
\hline FORWARD & 56.09 & 42.86 & TTTGACGACTACTGCAACTGT \\
\hline REVERSE & 54.55 & 42.86 & GAACTGGAATTGACATCAGTC \\
\hline FORWARD & 54.18 & 42.86 & ATAACCTCTACCATGTGCATC \\
\hline REVERSE & 55.4 & 50 & GTCGTATGGTGGAAACAGAC \\
\hline FORWARD & 55.4 & 50 & GTCTGTTTCCACCATACGAC \\
\hline REVERSE & 55.11 & 38.1 & TTGTTTCGAGATAGGAGTCAA \\
\hline FORWARD & 55.52 & 42.86 & CTGAAGATCTCCCATTCTTTC \\
\hline REVERSE & 55.19 & 50 & TCAGAGTCCGGTGAAGATAC \\
\hline FORWARD & 55.4 & 42.86 & CGAAGTTGATGGAGATACAGA \\
\hline REVERSE & 54.64 & 38.1 & ATGTGGTGAAGAAACTCCATA \\
\hline FORWARD & 55.86 & 47.62 & CTCTAAAAGGGCAGACTGTTC \\
\hline REVERSE & 54.8 & 47.62 & CTCTTCTGAGAGGCTCTTCTT \\
\hline FORWARD & 54.98 & 42.86 & CCAAAGAGAGTACATGAATCG \\
\hline REVERSE & 54.64 & 38.1 & TTACAAAGAGGTTTCAGTTGG \\
\hline FORWARD & 55.02 & 38.1 & GACAGATTTTAGCTTGCTTGA \\
\hline REVERSE & 54.04 & 38.1 & GCAAATTCGGAAGAAGTAGTA \\
\hline FORWARD & 54.71 & 28.57 & AAAATTATCGCCATCCTTAAT \\
\hline REVERSE & 54.68 & 38.1 & AAGAGGTCGGAGTGTTTTAAT \\
\hline FORWARD & 54.58 & 52.38 & CTAACССТСТСССТCAТАСТC \\
\hline REVERSE & 56.33 & 50 & GGGCTGATAGGGTTTGTAAG \\
\hline FORWARD & 55.51 & 42.86 & ATCAGGATATGTTGGGAGAAC \\
\hline REVERSE & 55.13 & 31.82 & AAACAACTGTTCCAAATCTTTC \\
\hline FORWARD & 55.15 & 47.62 & GTATAAGGAGAGGGTTCGAGA \\
\hline REVERSE & 55.03 & 38.1 & TACTTGTCCCTTTCACTTTCA \\
\hline FORWARD & 54.47 & 42.86 & TTCTCTCAGCTTCTCTATCCA \\
\hline REVERSE & 55.34 & 38.1 & ATGGATTCTGATAATGCCTCT \\
\hline FORWARD & 54.74 & 38.1 & CGAAGCTCTCATAATTTGTGT \\
\hline REVERSE & 54.83 & 38.1 & AGATCAAGGCGAGATCTATTT \\
\hline FORWARD & 55.26 & 38.1 & ATCTCGAATGGAGTTTATGGT \\
\hline REVERSE & 53.54 & 38.1 & ACTGGTAATGTTGAGATCCAT \\
\hline FORWARD & 54.4 & 38.1 & AACAAGTTGAAGCATATCCAC \\
\hline REVERSE & 55.25 & 52.38 & CTCGTCTGGTAGTATGCAGAG \\
\hline FORWARD & 56.63 & 50 & AGAGGATGATGAGGTCCAAG \\
\hline REVERSE & 54.64 & 38.1 & AGGTGAATTGACATGTAAGGA \\
\hline FORWARD & 56.33 & & GGCTGATAGGGTTTGTAAGG \\
\hline
\end{tabular}

\begin{tabular}{|c|c|c|}
\hline Prod size & Motif & \\
\hline 153 & TTTATT & \\
\hline 154 & TTTATT & \\
\hline & & \\
\hline 148 & TCTCTG & \\
\hline 142 & САССАA & \\
\hline & & \\
\hline 151 & CTCGAC & \\
\hline $170 \mathrm{k}$ & CTCCAC & \\
\hline $1 / 4$ & CILUAC & \\
\hline 152 & CGCCAC & \\
\hline 152 & GAGGAT & \\
\hline & & \\
\hline 141 & TTCCCT & \\
\hline 153 & ССТСТС & \\
\hline & & \\
\hline 169 & ССТСТC & \\
\hline 149 & GGAAAA & \\
\hline & & \\
\hline 146 & AAACCC & \\
\hline 171 & GTGAAG & \\
\hline & & \\
\hline 153 & AGGGAG & \\
\hline$\left.181\right|^{\prime}$ & ТСТССС & \\
\hline & & \\
\hline 145 & ACGAAG & \\
\hline & & \\
\hline 148 & ATGGAT & \\
\hline 136 & AACCCT & \\
\hline & & \\
\hline 147 & GAGGAT & \\
\hline & & \\
\hline $34 \mid$ & I & \\
\hline
\end{tabular}

Motif Len $\mid$ SSR 6 TTTATTTTTATTTTTATTTTTATTTTTATT

6 TTTATTTTTATTTTTATTTTTATT
6 TCTCTGTCTCTGTCTCTGTCTCTG 6 CACCAACACCAACACCAACACCAA 6 CTCGACCTCGACCTCGACCTCGAC 6 CTCGACCTCGACCTCGACCTCGACCTCGA 


\begin{tabular}{|c|c|c|c|c|c|c|c|c|c|}
\hline Locus_2920_Transcript_36/43_Confidence_0.586_Length_2898 & REVERSE & 54.58 & 52.38 & CTAACCCTCTCCCTCATACTC & & & & & \\
\hline Locus_3052_Transcript_19/32_Confidence_0.614_Length_3892 & FORWARD & 55.2 & 33.33 & ACTACCAAAACCCAATCAAAT & 152 & CAACTT & & CAACTTCAACTTCAACTTCAACTT & 24 \\
\hline Locus_3052_Transcript_19/32_Confidence_0.614_Length_3892 & REVERSE & 54.33 & 33.33 & TGCTATCATCTGAATGTGAAA & & & & & \\
\hline \begin{tabular}{|l} 
Locus_3171_Transcript_9/19_Confidence_0.657_Length_701 \\
\end{tabular} & FORWARD & 55.04 & 42.86 & CGTAAACCTTGATCTATGACG & 176 & CTCCCT & & СТСССТСТСССТСТСССТСТСССТ & 24 \\
\hline Locus_3171_Transcript_9/19_Confidence_0.657_Length_701 & REVERSE & 54.72 & 42.86 & GGGTCAAATATGGACTAAGGT & & & & & \\
\hline Locus_3213_Transcript_21/41_Confidence_0.368_Length_3819 & FORWARD & 54.96 & 42.86 & CACAGATCATTATAGGCCAAG & 156 & GGAGAG & & GGAGAGGGAGAGGGAGAGGGAGAGGGAC & 30 \\
\hline Locus_3213_Transcript_21/41_Confidence_0.368_Length_3819 & REVERSE & 55.05 & 40 & ATCATCATGGTCTGGTTCAT & & & & & \\
\hline Locus_3449_Transcript_24/52_Confidence_0.614_Length_9259 & FORWARD & 55.16 & 42.86 & TTCATCAGGAGAATCTGTCAC & 150 & CTCAAC & & СТСААССТСААССТСААССТСAАС & 24 \\
\hline Locus_3449_Transcript_24/52_Confidence_0.614_Length_9259 & REVERSE & 55.28 & 47.62 & GTGAAGCAGTAGTTGCAGAAC & & & & & \\
\hline Locus_4147_Transcript_11/25_Confidence_0.456_Length_2080 & FORWARD & 55.38 & 38.1 & AACTCACCTTCCTCAATGTTT & 154 & GGAGAA & & GGAGAAGGAGAAGGAGAAGGAGAA & 24 \\
\hline Locus_4147_Transcript_11/25_Confidence_0.456_Length_2080 & REVERSE & 55.22 & 40 & ATAATTTCCGCTTCTCCTTC & & & & & \\
\hline Locus_4451_Transcript_19/29_Confidence_0.662_Length_5917 & FORWARD & 55.37 & 42.86 & AGGAAGATGAGGTCAACAACT & 178 & AAGTTT & 6 & AAGTTTAAGTTTAAGTTTAAGTTTAAGTTT & 30 \\
\hline Locus_4451_Transcript_19/29_Confidence_0.662_Length_5917 & REVERSE & 54.88 & 38.1 & CAGCCTAAACAGAAAGATGAA & & & & & \\
\hline Locus_4451_Transcript_19/29_Confidence_0.662_Length_5917 & FORWARD & 55.37 & 42.86 & AGGAAGATGAGGTCAACAACT & 174 & AAGTTT & 6 & AAGTTTAAGTTTAAGTTTAAGTTTAAGTTT & 30 \\
\hline Locus_4451_Transcript_19/29_Confidence_0.662_Length_5917 & REVERSE & 55.06 & 38.1 & TCCACATGGAGACATTTTTAC & & & & & \\
\hline Locus_4473_Transcript_17/24_Confidence_0.561_Length_4523 & FORWARD & 56.43 & 45 & AGCAGCTTTCCAAACCTTAG & 138 & TCTCCT & & TCTCСТTCTCСТTCTCСТTCTCСТ & 24 \\
\hline Locus_4473_Transcript_17/24_Confidence_0.561_Length_4523 & REVERSE & 55.5 & 42.86 & AAGATCCAGCAGAAGAAGAAG & & & & & \\
\hline Locus_4627_Transcript_24/28_Confidence_0.656_Length_6324 & FORWARD & 55.84 & 47.62 & CССТАACCCTAATCCTATTCC & 181 & CATCGC & & CATCGCCATCGCCATCGCCATCGC & 24 \\
\hline Locus_4627_Transcript_24/28_Confidence_0.656_Length_6324 & REVERSE & 55.24 & 45 & ATAGTGAAGGAAGGGTCGAT & & & & & \\
\hline Locus_4864_Transcript_5/12_Confidence_0.655_Length_1238 & FORWARD & 55.07 & 42.86 & GATCAGATGGTAGTGCATGTT & 147 & TAAAAA & 6 & $\mathrm{~A}$ & 24 \\
\hline Locus_4864_Transcript_5/12_Confidence_0.655_Length_1238 & REVERSE & 54.67 & 33.33 & TGATTCGTTTTCAGACTTTTC & & & & & \\
\hline Locus_5631_Transcript_8/18_Confidence_0.588_Length_2004 & FORWARD & 55.01 & 38.1 & CCACCCATTCTATTTTCTCTT & 175 & TCCCCT & 6 & TCСССТTCСССТТССССТTССССТ & 24 \\
\hline Locus_5631_Transcript_8/18_Confidence_0.588_Length_2004 & REVERSE & 54.31 & 38.1 & GCCTGATTTGTCATTGATACT & & & & & \\
\hline Locus_5631_Transcript_8/18_Confidence_0.588_Length_2004 & FORWARD & 55.4 & 38.1 & TTCCCCTTTCAGAGTATCAAT & 153 & TCCСCT & & TCCCCTTCСCCTTCCССТTCСCCT & 24 \\
\hline Locus_5631_Transcript_8/18_Confidence_0.588_Length_2004 & REVERSE & 55.34 & 38.1 & GGAAATGAGAGAGCTTTTGTT & & & & & \\
\hline Locus_5631_Transcript_8/18_Confidence_0.588_Length_2004 & FORWARD & 55.47 & 47.62 & CCTTCCATCACCTTCTCTAAC & 148 & CCAATT & & CCAATTCCAATTCCAATTCCAATT & 24 \\
\hline Locus_5631_Transcript_8/18_Confidence_0.588_Length_2004 & REVERSE & 56.08 & 45 & AGGAGATTGGTTGTGGAGTT & & & & & \\
\hline Locus_5900_Transcript_29/35_Confidence_0.596_Length_1811 & FORWARD & 54.99 & 42.86 & CTCTGATAGGAAAATCCTGGT & 150 & CTCTCC & & СТСТСССТСТСССТСТСССТСТСС & 24 \\
\hline Locus_5900_Transcript_29/35_Confidence_0.596_Length_1811 & REVERSE & 54.33 & 47.62 & GGTAGTGCAGAGAACAGAAAG & & & & & \\
\hline Locus_5967_Transcript_22/43_Confidence_0.508_Length_1364 & FORWARD & 55.03 & 42.86 & GCCCTAAACCTCAATGTCTAT & 163 & TATGTC & & TATGTCTATGTCTATGTCTATGTC & 24 \\
\hline Locus_5967_Transcript_22/43_Confidence_0.508_Length_1364 & REVERSE & 55.08 & 50 & CTCCTAATCGCGACTGTATC & & & & & \\
\hline Locus_6009_Transcript_5/12_Confidence_0.655_Length_1634 & FORWARD & 55.04 & 47.62 & GACTTCCTGCTCTTTCTCTTC & 159 & GATCAC & 6 & GATCACGATCACGATCACGATCACGATCA & 30 \\
\hline Locus_6009_Transcript_5/12_Confidence_0.655_Length_1634 & REVERSE & 56.18 & 45 & AGCTTCCGACTTTGAATCTC & & & & & \\
\hline Locus_6071_Transcript_4/6_Confidence_0.690_Length_899 & FORWARD & 55.63 & 38.1 & AGATTTGGGTTCAATCATCTC & 164 & ACCAAA & & ACCAAAACCAAAACCAAAACCAAA & 24 \\
\hline Locus_6071_Transcript_4/6_Confidence_0.690_Length_899 & REVERSE & 55.18 & 47.62 & TGGTGGAGTTGGTAGTGATAG & & & & & \\
\hline Locus_6071_Transcript_4/6_Confidence_0.690_Length_899 & FORWARD & 55.18 & 47.62 & CTATCACTACCAACTCCACCA & 179 & ACCGCC & & ACCGCCACCGCCACCGCCACCGCC & 24 \\
\hline Locus_6071_Transcript_4/6_Confidence_0.690_Length_899 & REVERSE & 57.22 & 45 & TGGGATTATAACTGGGGATG & & & & & \\
\hline Locus_6725_Transcript_18/34_Confidence_0.601_Length_2029 & FORWARD & 55.31 & 33.33 & AAATCAGGTGGTTCAAATTCT & 148 & TGTGTT & & TGTGTTTGTGTTTGTGTTTGTGTT & 24 \\
\hline Locus_6725_Transcript_18/34_Confidence_0.601_Length_2029 & REVERSE & 54.96 & 28.57 & AAACAACTCAAAAACCAACAA & & & & & \\
\hline Locus_7607_Transcript_9/11_Confidence_0.619_Length_1489 & FORWARD & 54.83 & 38.1 & CCCATACAATCTCCCTTTAAT & 151 & CTCACC & & СТСАСССТСАСССТСАСССТСАСC & 24 \\
\hline Locus_7607_Transcript_9/11_Confidence_0.619_Length_1489 & REVERSE & 54.9 & 38.1 & CATTTAAGCTCAACTGCTCAT & & & & & \\
\hline Locus_7616_Transcript_14/41_Confidence_0.520_Length_1640 & FORWARD & 54.94 & 38.1 & ATTTCTCTGCTGACCTTCTTT & 139 & CTTCCT & & СTTCСТCTTCСТCTTCСТCTTCСТ & 24 \\
\hline Locus_7616_Transcript_14/41_Confidence_0.520_Length_1640 & REVERSE & 54.97 & 47.62 & ACAAGATCAGAGGAGGATAGG & & & & & \\
\hline Locus_7907_Transcript_33/39_Confidence_0.489_Length_2945 & FORWARD & 54.82 & 42.86 & ACCCATCAAAGATCTACCTTC & 151 & CACCTC & & САССТССАССТССАССТССАССТССАССТС & 36 \\
\hline
\end{tabular}




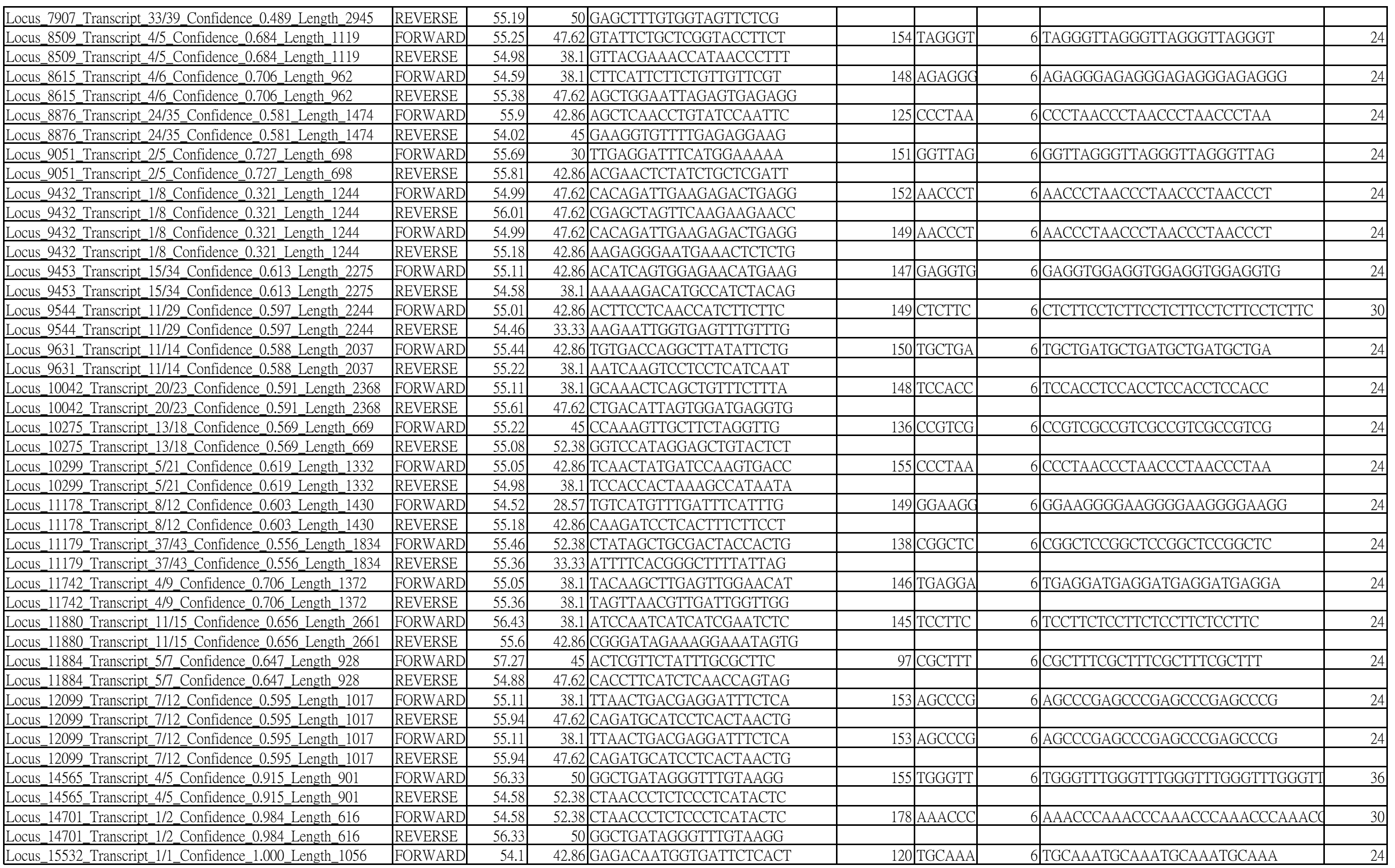




\begin{tabular}{|c|c|c|c|c|c|c|c|c|c|}
\hline Locus_15532_Transcript_1/1_Confidence_1.000_Length_1056 & REVERSE & 55.32 & 28.57 & TTTTGTCTGCTTTTGCATTAT & & & & & \\
\hline Locus_15533_Transcript_1/1_Confidence_1.000_Length_1056 & FORWARD & 54.1 & 42.86 & GAGACAATGGTGATTCTCACT & 120 & TGCAAA & 6 & TGCAAATGCAAATGCAAATGCAAA & 24 \\
\hline Locus_15533_Transcript_1/1_Confidence_1.000_Length_1056 & REVERSE & 55.32 & 28.57 & TTTTGTCTGCTTTTGCATTAT & & & & & \\
\hline Locus_15535_Transcript_1/1_Confidence_1.000_Length_354 & FORWARD & 54.1 & 42.86 & GAGACAATGGTGATTCTCACT & 120 & TGCAAA & & TGCAAATGCAAATGCAAATGCAAA & 24 \\
\hline Locus_15535_Transcript_1/1_Confidence_1.000_Length_354 & REVERSE & 55.32 & 28.57 & TTTTGTCTGCTTTTGCATTAT & & & & & \\
\hline Locus_15536_Transcript_1/1_Confidence_1.000_Length_354 & FORWARD & 54.1 & 42.86 & GAGACAATGGTGATTCTCACT & 120 & TGCAAA & 6 & TGCAAATGCAAATGCAAATGCAAA & 24 \\
\hline Locus_15536_Transcript_1/1_Confidence_1.000_Length_354 & REVERSE & 55.32 & 28.57 & TTTTGTCTGCTTTTGCATTAT & & & & & \\
\hline Locus_15540_Transcript_1/1_Confidence_1.000_Length_401 & FORWARD & 54.1 & 42.86 & GAGACAATGGTGATTCTCACT & 120 & TGCAAA & 6 & TGCAAATGCAAATGCAAATGCAAA & 24 \\
\hline Locus_15540_Transcript_1/1_Confidence_1.000_Length_401 & REVERSE & 55.32 & 28.57 & TTTTGTCTGCTTTTGCATTAT & & & & & \\
\hline Locus_15541_Transcript_1/1_Confidence_1.000_Length_401 & FORWARD & 54.1 & 42.86 & GAGACAATGGTGATTCTCACT & 120 & TGCAAA & 6 & TGCAAATGCAAATGCAAATGCAAA & 24 \\
\hline Locus_15541_Transcript_1/1_Confidence_1.000_Length_401 & REVERSE & 55.32 & 28.57 & TTTTGTCTGCTTTTGCATTAT & & & & & \\
\hline Locus_15767_Transcript_1/1_Confidence_1.000_Length_1603 & FORWARD & 56.1 & 38.1 & GCTTCAACAATCCTTTCAAGT & 163 & CACCAA & 6 & CACCAACACCAACACCAACACCAA & 24 \\
\hline Locus_15767_Transcript_1/1_Confidence_1.000_Length_1603 & REVERSE & 54.55 & 42.86 & GAACTGGAATTGACATCAGTC & & & & & \\
\hline Locus_15997_Transcript_1/1_Confidence_1.000_Length_1291 & FORWARD & 55.45 & 28.57 & CCAAAAAGAACACAAAAATCA & 171 & CTTCCT & 6 & СТTCСТCTТССТСТTССТСТTCСТ & 24 \\
\hline Locus_15997_Transcript_1/1_Confidence_1.000_Length_1291 & REVERSE & 55.23 & 42.86 & GAGGAAGAAGAGGATGAAGAA & & & & & \\
\hline Locus_18109_Transcript_1/1_Confidence_1.000_Length_735 & FORWARD & 54.58 & 52.38 & CTAАСССТСТСССТСAТАCТC & 151 & AAACCC & 6 & AAACCCAAACCCAAACCCAAACCCAAACC & 36 \\
\hline Locus_18109_Transcript_1/1_Confidence_1.000_Length_735 & REVERSE & 56.33 & 50 & GGCTGATAGGGTTTGTAAGG & & & & & \\
\hline Locus_18110_Transcript_1/1_Confidence_1.000_Length_761 & FORWARD & 54.58 & 52.38 & CTAАСССТСТСССТСАТАСТC & 151 & AAACCC & 6 & AAACCCAAACCCAAACCCAAACCCAAACC & 36 \\
\hline Locus_18110_Transcript_1/1_Confidence_1.000_Length_761 & REVERSE & 56.33 & 50 & GGCTGATAGGGTTTGTAAGG & & & & & \\
\hline Locus_18111_Transcript_1/1_Confidence_1.000_Length_746 & FORWARD & 55.26 & 52.38 & СТСАТАССТСССТСАТАСТСС & 150 & AAACCC & 6 & AAACCCAAACCCAAACCCAAACCCAAACC & 36 \\
\hline Locus_18111_Transcript_1/1_Confidence_1.000_Length_746 & REVERSE & 56.33 & 50 & GGCTGATAGGGTTTGTAAGG & & & & & \\
\hline Locus_18112_Transcript_1/1_Confidence_1.000_Length_721 & FORWARD & 54.58 & 52.38 & CTAАСССТСТСССТСAТАСТC & 151 & AAACCC & 6 & AAACCCAAACCCAAACCCAAACCCAAACC & 36 \\
\hline Locus_18112_Transcript_1/1_Confidence_1.000_Length_721 & REVERSE & 56.33 & 50 & GGCTGATAGGGTTTGTAAGG & & & & & \\
\hline Locus_18678_Transcript_1/1_Confidence_1.000_Length_459 & FORWARD & 55.38 & 42.86 & AGTATTCCTTAGCCATTACGC & 154 & ACGAAG & 6 & ACGAAGACGAAGACGAAGACGAAG & 24 \\
\hline \begin{tabular}{|l} 
Locus_18678_Transcript_1/1_Confidence_1.000_Length_459 \\
\end{tabular} & REVERSE & 54.7 & 42.86 & CCACTGGATCAATATGATAGC & & & & & \\
\hline Locus_18679_Transcript_1/1_Confidence_1.000_Length_458 & FORWARD & 55.38 & 42.86 & AGTATTCCTTAGCCATTACGC & 160 & ACGAAG & 6 & ACGAAGACGAAGACGAAGACGAAG & 24 \\
\hline Locus_18679_Transcript_1/1_Confidence_1.000_Length_458 & REVERSE & 54.7 & 42.86 & CCACTGGATCAATATGATAGC & & & & & \\
\hline Locus_18680_Transcript_1/1_Confidence_1.000_Length_452 & FORWARD & 55.38 & 42.86 & AGTATTCCTTAGCCATTACGC & 154 & ACGAAG & & ACGAAGACGAAGACGAAGACGAAG & 24 \\
\hline Locus_18680_Transcript_1/1_Confidence_1.000_Length_452 & REVERSE & 54.7 & 42.86 & CCACTGGATCAATATGATAGC & & & & & \\
\hline Locus_20202_Transcript_1/1_Confidence_1.000_Length_219 & FORWARD & 54.88 & 38.1 & TTCTTGACTGAGCTTATTTGG & 152 & AGGAAG & 6 & AGGAAGAGGAAGAGGAAGAGGAAG & 24 \\
\hline Locus_20202_Transcript_1/1_Confidence_1.000_Length_219 & REVERSE & 55.45 & 28.57 & TTTTTCATGGTTTTCTTTGTG & & & & & \\
\hline Locus_20799_Transcript_1/1_Confidence_1.000_Length_318 & FORWARD & 54.99 & 38.1 & ATATCACATGCTTGTCTGCTT & 140 & TTTGCA & & TTTGCATTTGCATTTGCATTTGCA & 24 \\
\hline Locus_20799_Transcript_1/1_Confidence_1.000_Length_318 & REVERSE & 56.09 & 42.86 & AGACAATGGTGAGGTGATTCT & & & & & \\
\hline Locus_20800_Transcript_1/1_Confidence_1.000_Length_322 & FORWARD & 55.32 & 28.57 & TTTTGTCTGCTTTTGCATTAT & 157 & TTTGCA & 6 & TTTGCATTTGCATTTGCATTTGCA & 24 \\
\hline Locus_20800_Transcript_1/1_Confidence_1.000_Length_322 & REVERSE & 56.09 & 42.86 & AATGGTGAGGTGATTCTCACT & & & & & \\
\hline Locus_20801_Transcript_1/1_Confidence_1.000_Length_324 & FORWARD & 55.32 & 28.57 & TTTTGTCTGCTTTTGCATTAT & 157 & TTTGCA & 6 & TTTGCATTTGCATTTGCATTTGCA & 24 \\
\hline Locus_20801_Transcript_1/1_Confidence_1.000_Length_324 & REVERSE & 56.09 & 42.86 & AATGGTGAGGTGATTCTCACT & & & & & \\
\hline Locus_20802_Transcript_1/1_Confidence_1.000_Length_342 & FORWARD & 55.32 & 28.57 & TTTTGTCTGCTTTTGCATTAT & 165 & TTTGCA & 6 & TTTGCATTTGCATTTGCATTTGCA & 24 \\
\hline Locus_20802_Transcript_1/1_Confidence_1.000_Length_342 & REVERSE & 54.88 & 47.62 & CTAGTGAGAATCACCCAAGTG & & & & & \\
\hline Locus_20803_Transcript_1/1_Confidence_1.000_Length_348 & FORWARD & 55.32 & 28.57 & TTTTGTCTGCTTTTGCATTAT & 165 & TTTGCA & & TTTGCATTTGCATTTGCATTTGCA & 24 \\
\hline Locus_20803_Transcript_1/1_Confidence_1.000_Length_348 & REVERSE & 54.88 & 47.62 & CTAGTGAGAATCACCCAAGTG & & & & & \\
\hline Locus_20804_Transcript_1/1_Confidence_1.000_Length_318 & FORWARD & 54.69 & 33.33 & TGCATTATATCACATGTTTGC & 146 & TTTGCA & & TTTGCATTTGCATTTGCATTTGCA & 24 \\
\hline Locus_20804_Transcript_1/1_Confidence_1.000_Length_318 & REVERSE & 55.18 & 38.1 & TGACAATGGTGATTCTTTCTC & & & & & \\
\hline Locus_20805_Transcript_1/1_Confidence_1.000_Length_224 & FORWARD & 55.32 & 28.57 & TTTTGTCTGCTTTTGCATTAT & 144 & TTTGCA & & TTTGCATTTGCATTTGCATTTGCA & 4 \\
\hline
\end{tabular}




\begin{tabular}{|l|l|r|r|l|r|r|r|r|}
\hline Locus_20805_Transcript_1/1_Confidence_1.000_Length_224 & REVERSE & 55.09 & 38.1 & TGAATGGTGATTCTCATTCTC & & & \\
\hline Locus_21213_Transcript_1/1_Confidence_1.000_Length_202 & FORWARD & 55.45 & 28.57 & TTTTTCATGGTTTTCTTTGTG & 123 & TCTTCC & 6 TCTTCCTCTTCCTCTTCCTCTTCC \\
\hline Locus_21213_Transcript_1/1_Confidence_1.00__Length_202 & REVERSE & 54.88 & 38.1 & TTCTTGACTGAGCTTATTTGG & & & 24 \\
\hline
\end{tabular}

